\title{
Identifikation und Optimierung im Kontext technischer Anwendungen
}

\author{
Von der Fakultät für Maschinenbau der \\ Technischen Universität Chemnitz \\ genehmigte
}

\section{Dissertation}

zur Erlangung des akademischen Grades

Doktoringenieur

(Dr.-Ing.)

vorgelegt

von Dipl.-Ing. Dirk Schellenberg geboren am 29.06.1978 in Freiberg(Sachs)

eingereicht am 22.06.2015

Gutachter:

Prof. Dr.-Ing. habil. Jörn Ihlemann

Prof. Dr. rer. nat. Roland Herzog

Tag der Verteidigung: 6. November 2015 



\section{Vorwort}

Die vorliegende Dissertation entstand während meiner Tätigkeit als wissenschaftlicher Mitarbeiter an der Professur Festkörpermechanik des Instituts für Mechanik und Thermodynamik der Technischen Universität Chemnitz sowie am Deutschen Institut für Kautschuktechnologie e.V. in Hannover. Für die während dieser Zeit erhaltene Unterstützung möchte ich mich an dieser Stelle gerne bedanken.

Mein besonderer Dank gilt meinem Doktorvater und Erstgutachter Herrn Prof. Jörn Ihlemann, dessen Ideen die Grundlage eines maßgeblichen Anteils der Arbeit darstellen und ohne dessen vielfältige Unterstützung die Fertigstellung der Dissertation nicht möglich gewesen wäre. Die Vielzahl fachlicher Gespräche und Diskussionen habe ich sehr genossen und eröffnete mir oft neue Blickwinkel und Perspektiven. Für das entgegengebrachte Vertrauen und den uneingeschränkten Rückhalt, insbesondere in schwierigen Situationen, möchte ich mich herzlich bedanken.

Ebenso möchte ich Herrn Prof. Reiner Kreißig, dem vormaligen Inhaber der Professur Festkörpermechanik, ausdrücklich für die Möglichkeit zur wissenschaftlichen Arbeit auf einem außerordentlich interessanten Gebiet sowie die aktive Förderung während dieser Zeit danken. Es ist vor allem seiner intensiven Unterstützung während der Übergabe der Professur an Herrn Prof. Ihlemann geschuldet, dass ich meine Arbeit fortsetzen und die Dissertation erfolgreich abschließen konnte. Als Mentor hat er mich sowohl fachlich als auch persönlich inspiriert und mich auch nach seinem Ausscheiden aus dem universitären Betrieb begleitet.

Ein großer Dank gebührt meinen Kollegen und Studenten für die intensive Zusammenarbeit, welche mir nicht nur fachlich, sondern auch persönlich sehr viel bedeutet hat. Durch die interessanten fachlichen Gespräche konnte ich nicht nur Einblick in andere Fachgebiete gewinnen, sondern bekam auch wesentliche Impulse und Unterstützung für meine eigene wissenschaftliche Arbeit. Besonders hervorheben möchte ich hierbei Hans Wulf, welcher eine unschätzbare Unterstützung bei der Weiterentwicklung von SPC-Opt und auch abseits fachlicher Themen jederzeit ein spannender Gesprächspartner war. Zudem möchte ich mich bei Niklas Nostitz für seine Hilfe bei der Verbesserung der Optimierungsverfahren bedanken und wünsche ihm als meinem Projektnachfolger alles Gute.

Herrn Prof. Roland Herzog danke ich für die Übernahme des Zweitgutachtens sowie die damit verbundenen Mühen und sein Interesse an meiner Arbeit. Zudem möchte ich mich bei Herrn Prof. Dirk Landgrebe für die freundliche Übernahme des Prüfungsvorsitzes bedanken. Schließlich möchte ich mich ganz herzlich bei meiner Frau Julia für die ihre langjährige Unterstützung, ihre Geduld und ihre Motivation zur Fertigstellung der Dissertation bedanken. Für die Zeit, die ich abends oder am Wochenende im Büro verbracht habe und ich nicht mit ihr oder meinen Kindern Maja und Leo verbringen konnte, kann ich mich nur entschuldigen. 


\section{Inhaltsverzeichnis}

Formelzeichen, Symbole und Abkürzungen $\quad$ VII

Kurzfassung $\quad$ XI

\begin{tabular}{lll}
\hline Abstract & XII
\end{tabular}

1 Einleitung 1

2 Das Programm SPC-Opt 4

2.1 Anforderungsprofil und Funktionalität von $S P C-O p t \ldots \ldots \ldots$

2.2 Programmstruktur . . . . . . . . . . . . . . . . . . 6

2.3 Optimieren . . . . . . . . . . . . . . . . . . . . . . . . . . 8

2.4 FEM-Schnittstellen . . . . . . . . . . . . . . . . . . . . . . . . 10

2.5 Parametrisierung der Modellgeometrie . . . . . . . . . . . . . . . . . . 14

2.6 Parallelisierung $\ldots \ldots \ldots \ldots \ldots$

$\begin{array}{lll}3 & \text { Parameteridentifikation } & 22\end{array}$

3.1 Optimierungsaufgabe . . . . . . . . . . . . . . . . . . . . . . . 22

3.2 Gewichtete Fehlerquadratkennzahl (GFK) . . . . . . . . . . . . . . . . . . 24

3.3 Gradientenverfahren (Verfahren des steilsten Abstiegs) . . . . . . . . . . . . . 25

3.4 Newton-Verfahren . . . . . . . . . . . . . . . . . . . . . . . 25

3.5 Gauss-Newton-Verfahren . . . . . . . . . . . . . . . . . . . . . . 26

3.6 Levenberg-Marquardt-Verfahren . . . . . . . . . . . . . . . . . . . . . . . 26

3.6 .1 Levenberg-Marquardt als Trust-Region-Verfahren . . . . . . . . . . . . . . 27

3.6 .2 Modifikation nach Fletchen . . . . . . . . . . . . . . . . . . . . . . 28

3.7 Berücksichtigung von Restriktionen . . . . . . . . . . . . . . . . . . . . 31

3.8 Abbruchbedingungen . . . . . . . . . . . . . . . . . . . . . . . 35

3.9 Parameterschätzung . . . . . . . . . . . . . . . . . . . . . . 37

3.10 Formidentifikation . . . . . . . . . . . . . . . . . 40

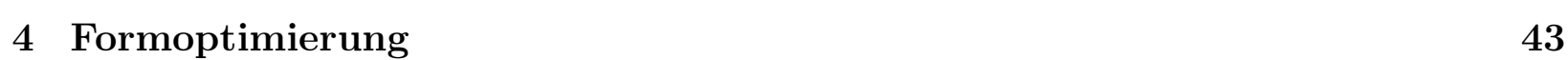

4.1 Trust-Region-Verfahren . . . . . . . . . . . . . . . . . . . . . . 43

4.2 Quasi-Newton-Verfahren . . . . . . . . . . . . . . . . . . . . 46

4.3 Bewertung der Trust-Region-Verfahren . . . . . . . . . . . . . . . . . . . . . . 48

4.4 Beispiel Lochscheibe . . . . . . . . . . . . . . . . . . . . . . . . . . . . . . . . . 49 
5 Startparametervariation $\quad 52$

5.1 Methoden zur Erzeugung von Startvektoren . . . . . . . . . . . . . . . . . 52

5.2 Optimierung der Startdesigns . . . . . . . . . . . . . . . . . 55

5.2 .1 Designkriterien . . . . . . . . . . . . . . . . 56

5.2 .2 Stochastische Optimierungsverfahren . . . . . . . . . . . . . . 60

6 Identifikation von Materialparametern anhand bauteilnaher Probekörper 63

6.1 Problemstellung . . . . . . . . . . . . . . . . . . . . 63

6.2 Bauteilgeometrie und Belastungen . . . . . . . . . . . . . . . . . 64

6.3 Gummimischung . . . . . . . . . . . . . . . . . . . . . . . 68

6.4 Stoffgesetze . . . . . . . . . . . . . . . . . . . . . . 69

6.4 MORPH in repräsentativen Richtungen . . . . . . . . . . . . . . . . . 73

6.4 .2 Verwendung von Stabilisierungselementen . . . . . . . . . . . . . . 75

6.5 Bauteilsimulation . . . . . . . . . . . . . . . . 77

6.6 Identifikation mit Standardprobekörpern . . . . . . . . . . . . . . . . . . . . . 82

6.6 .1 Auswahl der Probekörpergeometrien . . . . . . . . . . . . . . . . . . 83

6.6 .2 Nullpunktverschiebung von Messdaten . . . . . . . . . . . . . . . . . . . . 88

6.6 .3 Kriterien zum Vergleich der Lastfälle . . . . . . . . . . . . . . . . . . . . . . . 92

6.6 .4 Durchführung der Identifikation . . . . . . . . . . . . . . . . . . . . . . . 99

6.7 Identifikation anhand bauteilnaher Probekörper . . . . . . . . . . . . . . . 105

6.7 .1 Der bauteilnahe Probekörper . . . . . . . . . . . . . . . . . . . 108

6.7 .2 Simulation der Lastfälle am bauteilnahen Probekörper . . . . . . . . . . . . . 110

6.7 .3 Inhomogen-Parameteridentifikation . . . . . . . . . . . . . . 116

6.8 Validierung am Bauteil . . . . . . . . . . . . . . . . . . . . . . 122

7 Zusammenfassung und Ausblick $\quad 125$

\begin{tabular}{lr}
\hline Anhang & 129
\end{tabular}

A.1 Software und Programmpakete . . . . . . . . . . . . . . . . . . . . . . 129

A.2 Visualisierung von FEM-Ergebnissen . . . . . . . . . . . . . . . . . . . . 133

\begin{tabular}{ll}
\hline Literaturverzeichnis & 135
\end{tabular} 


\title{
Formelzeichen, Symbole und Abkürzungen
}

\author{
Abkürzungen \\ CHiC Chemnitzer High Performance Cluster \\ EPDM Ethylene Probylene Diene Monomer \\ (Ethylen-Propylen-Dien-Kautschuk) \\ FEM Finite-Elemente-Methode \\ FQ Fortschrittsquotient \\ FQM Fehlerquadratminimierung \\ GFK Gewichtete Fehlerquadrat-Kennzahl \\ $\overline{\text { GFK }} \quad$ kombinierte Gewichtete Fehlerquadrat-Kennzahl \\ HPC High Performance Cluster \\ LHD Latin Hypercube Design \\ MPI Message Passing Interface \\ Morph Model of Rubber Phenomenology \\ MPP massively parallel processor computer \\ NoCs Networks of independent Computers \\ NR Natural Rubber (Naturkautschuk) \\ PE Processing Element \\ RD Random Design \\ SBR Styrene Butadiene Rubber (Styrol-Butadien-Kautschuk) \\ SLHD Symmetric Latin Hypercube Design
}

Skalare

Symbol Beschreibung

\begin{tabular}{llr}
\hline$a_{j}$ & Bildungsvorschrift einer Abbruchbedingung & (Gl. 3.37) \\
$\alpha_{t}$ & Wärmeausdehnungskoeffizient & (Kap. 6.7.2) \\
$C_{\mathrm{T}}$ & TresCA-Invariante von $\stackrel{G}{\underline{G}}$ & (Gl. 6.6) \\
$C_{\mathrm{T}}^{\mathrm{S}}$ & Schleppzeiger im MorPH-Stoffgesetz & (Gl. [6.6) \\
$\gamma_{1}, \gamma_{2}$ & Streckenverhältnisse zur Positionsbestimmung von & (Gl. 2.3) \\
$d_{\eta 1}, d_{\eta 2}$ & FEM-Knoten bei Netzaktualisierung & Abstände eines FEM-Knotens zu den Bezugskurven in \\
$d_{\xi 1}, d_{\xi 2}$ & $\eta$-Richtung & Abstände eines FEM-Knotens zu den Bezugskurven in
\end{tabular}

$\xi$-Richtung 


\begin{tabular}{|c|c|c|}
\hline$\Delta$ & Trust-Region-Radius & (Gl. 4.2) \\
\hline$E^{A}$ & Teilabbruchbedingung & (Gl. 3.37) \\
\hline $\bar{E}^{A}$ & kombiniertes Abbruchkriterium & (Gl. 3.39) \\
\hline$\varepsilon$ & technische Dehnung & (Kap. 6.6.1) \\
\hline$\varepsilon^{A}$ & Abbruchschranke & (Gl. 3.36) \\
\hline $\bar{\varepsilon}^{A}$ & definierte Abbruchschranke für Optimierung & (Kap. 3.8) \\
\hline$\varepsilon_{e q}$ & Vergleichsdehnung & (Kap. 6.5) \\
\hline$\varepsilon_{e q}^{p}$ & plastische Vergleichsdehnung & (Kap. 6.5) \\
\hline$\Phi$ & Zielfunktion & (Gl. 3.4) \\
\hline$h_{i}$ & HENKY-Dehnungen & (Gl. 6.15) \\
\hline$J_{3}$ & Volumenänderung & (Gl. 6.12) \\
\hline $\begin{array}{l}G \\
L \\
\mathrm{~T}\end{array}$ & TRESCA-Invariante von $\stackrel{G}{\underline{\underline{L}}}$ & (Gl. 6.8) \\
\hline$K$ & Kompressionsmodul & (Kap. 6.7.2) \\
\hline$\kappa, \gamma$ & Schermaß & $(\mathrm{Gl} .6 .6 .2)$ \\
\hline$l, l_{0}$ & aktuelle Länge, Ausgangslänge & (Gl. 6.17) \\
\hline$\lambda_{i}$ & Hauptstreckungen & (Gl. 6.4) \\
\hline $\bar{\lambda}$ & Umformgrad & (Gl. 6.39) \\
\hline$\lambda^{L}$ & Wichtungsfaktor im Levenberg-Marquardt-Algorithmus & (Gl. 3.22) \\
\hline$\lambda_{C}^{L}$ & Cut-Off-Parameter & (Gl. 3.27) \\
\hline$m$ & Quadratisches Ersatzmodell in Form einer Taylor-Reihe & (Gl. 3.13) \\
\hline$M$ & Modell zur Beschreibung eines Zustandes am Bauteil & (Gl. 2.3) \\
\hline$\nu^{F}$ & allgemeiner Fletcher-Parameter & (Gl. 3.26) \\
\hline$p$ & Designvariable & (Gl. 2.1) \\
\hline$q$ & Wichtungsfaktor auf Basis von $J_{3}$ & (Gl. 6.12) \\
\hline $\bar{r}_{i}$ & Residuum zwischen Modell und fehlerbehaftetem Messwert & (Gl. 3.1) \\
\hline$r_{i}$ & einheitenloses Residuum $\bar{r}_{i}$, bei Bedarf skaliert & (Gl. 3.4) \\
\hline$\rho$ & Fortschrittsquotient & (Gl. 3.25) \\
\hline$\dot{s}$ & akkumulierte Verzerrungsgeschwindigkeit & (Gl. 6.41) \\
\hline$s^{2}$ & Stichprobenvarianz & (Gl. 3.46) \\
\hline$\sigma$ & technische Spannung & (Kap. 6.6.1) \\
\hline$t_{i}$ & Beobachtungszeitpunkt & (Gl. 3.1) \\
\hline$\tau$ & Schubspannung & (Kap. 6.6.2) \\
\hline$\hat{W}$ & Energiedichte & (Gl. 6.1) \\
\hline$w_{i}$ & Wichtungsfaktor der Residuen & (Gl. 3.4) \\
\hline$w_{j}$ & Wichtungsfaktor der Abbruchbedingungen & (Gl. 3.37) \\
\hline $\bar{y}_{i}$ & fehlerbehafteter Messwert & (Gl. 3.1) \\
\hline
\end{tabular}




\section{Vektoren}

\begin{tabular}{|c|c|c|}
\hline Symbol & Beschreibung & \\
\hline$d_{k}$ & Korrektur-Vektor in Schritt $k$ & (Gl. 3.8) \\
\hline$d_{k}^{L M}$ & Levenberg-Marquardt-Korrekturschritt & (Gl. 3.12) \\
\hline$d_{k}^{N}$ & Newton-Korrekturschritt & (Gl. 3.23) \\
\hline$\underline{p}$ & Parametervektor & (Gl. 3.1) \\
\hline$\underline{R}_{i}^{B}$ & $\begin{array}{l}\text { Koordinaten eines betrachteten Messpunktes zum Zeitpunkt } \\
t_{i}\end{array}$ & (Gl. 2.1) \\
\hline$\underline{R}^{0}$ & Anfangskoordinaten der FEM-Knoten & (Gl. 2.1) \\
\hline$\underline{R}$ & Vektor der neu berechneten Koordinaten der FEM-Knoten & (Gl. 2.1) \\
\hline$\underline{T}$ & Formbasisvektor & (Gl. 2.1) \\
\hline$\underline{x}$ & Parametervektor in allgemeiner mathematischer Form & (Gl. 3.5) \\
\hline$\underline{x}_{A}$ & letzte akzeptierte Lösung & $(\mathrm{Gl} .3 .40)$ \\
\hline
\end{tabular}

\section{Matrizen und Tensoren}

\begin{tabular}{|c|c|c|}
\hline Symbol & Beschreibung & \\
\hline$B$ & Approximierte Hesse-Matrix & (Gl. 3.15) \\
\hline$\underline{\underline{C}}$ & Rechts-CAUCHY-GreEn-Tensor & (Gl. 6.5) \\
\hline$\underline{\underline{G}}$ & Gestaltänderungsanteil von $\underline{\underline{C}}$ & (Gl. 6.5) \\
\hline$D$ & Skalierungsmatrix & $(\mathrm{Gl} .4 .2)$ \\
\hline$\underline{\underline{D}}$ & Tensor der Formänderungsgeschwindigkeiten & (Gl. 6.39) \\
\hline$\underline{\underline{F}}$ & Deformationsgradient & (Gl. 6.40) \\
\hline$H, H^{r e d}$ & Hesse-Matrix, reduzierte Hesse-Matrix & (Gl. 3.34) \\
\hline$I$ & Einheitsmatrix & (Gl. 3.19) \\
\hline$\underline{\underline{J}}, J$ & Jakobi-Matrix & (Gl. 3.6) \\
\hline$K$ & Korrelationsmatrix & (Gl. 3.51) \\
\hline$\underline{\underline{L}}$ & Geschwindigkeitsgradient & (Gl. 6.40) \\
\hline $\begin{array}{l}\underline{G} \\
\underline{\underline{L}}\end{array}$ & $\begin{array}{l}\text { LAGRANGEsches Äquivalent zur ZAREMBA-JAUMANN- } \\
\text { Zeitableitung des isochoren Links-CAUCHY-GREEN-Tensor }\end{array}$ & (Gl. 6.8) \\
\hline$P$ & Kovarianzmatrix & (Gl. 3.47) \\
\hline$\Pi$ & Permutationsmatrix & (Kap. 5.1) \\
\hline$\underline{\underline{T}}$ & 1. Piola-Kirchhoff-Spannungstensor & (Gl. 6.16) \\
\hline$\underline{\underline{T}}$ & 2. Piola-Kirchhoff-Spannungstensor & (Gl. 6.5) \\
\hline$\underline{\underline{T}}^{Z}, \underline{\underline{T}}^{H}$ & Zusatz- und Hüllspannungen & (Gl. 6.7) \\
\hline$\underline{\underline{U}}$ & Rechter Strecktensor & (Kap. 6.6.3) \\
\hline
\end{tabular}




\section{Operatoren und sonstige Symbole}

Symbol Beschreibung

\begin{tabular}{ll}
\hline()$^{T}$ & Transponierter Tensor \\
()$^{-1}$ & Inverser Tensor \\
$\nabla$ & Nabla-Operator \\
$d()$ & Vollständiges Differential \\
$\partial() / \partial()$ & Partielle Ableitung \\
$S p()$ & Spur eines Tensors
\end{tabular}




\section{Kurzfassung}

Es wurde die Optimierungssoftware SPC-Opt entwickelt, mit welcher sich Aufgaben aus den Bereichen der Formoptimierung sowie der Material- und Formidentifikation bearbeiten lassen. Zur Lösung von Identifikationsproblemen steht eine robuste Implementierung des Levenberg-Marquardt-Fletcher-Verfahrens zur Verfügung. Ergänzt wird dieses durch LineSearch- und Trust-Region-Verfahren, welche sich besonders für Aufgaben der Formoptimierung eignen. Es wurden effiziente Algorithmen zur Approximation der Hesse-Matrix sowie verschiedene Verfahren zur Startparametervariation integriert. Das Programm verfügt über Schnittstellen zur Nutzung von ABAQUS, ANSYS, MSC.MARC, eigenen FEM-Programmen sowie LUA-Skripten. Für Formoptimierungen können geometrische Konturen durch NURBS approximiert und deren Kontrollpunkte als Formparameter genutzt werden. Die Aktualisierung der FEM-Netze entsprechend der Formparameteränderung erfolgt durch ein analytisches Verfahren. Der zweite Schwerpunkt der Arbeit bezieht sich auf die Weiterentwicklung bestehender Verfahren zur Materialparameteridentifikation im Bereich der Gummiwerkstoffe. Hierbei wurde das Konzept der Anpassung anhand bauteilnaher Probekörper entwickelt. Dabei wurde am Beispiel einer Fahrwerksbuchse ein Probekörper entworfen, welcher dem originalen Bauteil zwar ähnlich sieht, jedoch eine deutlich einfachere Geometrie hat. Durch diesen konnte das Verhalten des Bauteils gut approximiert und sichergestellt werden, dass die im Rahmen der Parameteridentifikation durchgeführten FEM-Simulationen sicher konvergieren. Zudem wurden die Nutzerschnittstellen des inelastischen MORPH-Stoffgesetz für MSC.MARC und ABAQUS weiterentwickelt, sodass diese nunmehr auch im industriellen Umfeld nutzbar sind. Es konnte nachgewiesen werden, dass die Verwendung bauteilnah identifizierter Parameter zu einer erheblich besseren Abbildung des Materialverhaltens führt als die Verwendung anhand von Standardprobekörpern identifizierter Parameter. Weiterhin zeigte sich, dass vor allem der Einsatz eines Stoffgesetzes mit der Möglichkeit zur Abbildung des charakteristischen Verhaltens von Elastomeren unbedingt erforderlich ist.

\section{Schlagworte}

Stoffgesetzanpassung, Parameteridentifikation, Formoptimierung, Formidentifikation, Gummiwerkstoffe, Nichtlineare FEM, Materialcharakterisierung, Numerische Integration 


\section{Abstract}

Within the scope of this work the optimization software SPC-Opt has been developed to successfully process tasks in the fields of shape optimization and parameter identification. The software includes a robust Levenberg-Marquardt-Fletcher algorithm, several line search and trust region algorithms as well as efficient methods for the approximation of the Hessian matrix. Additionally, procedures for the variation of initial parameters (Design Of Experiments) were implemented. The software includes interfaces to ABAQUS, ANSYS, MSC.MARC, inhouse FEM programs and LUA scripts. Within shape optimization problems, geometric shapes are approximated by NURBS and the related control points are employed as design variables. For the update of the FE mesh during the variation of the design variables, a special analytical algorithm is used to preserve the mesh topology.

Another focus is related to the further development of existing material parameter identification procedures for rubber materials. Therefor, the concept of component-oriented specimens was developed. Using the example of a bushing, a specimen was designed, which is similar to the original component but has a much simpler geometry. According to this, the behavior of the original component is approximated and the stability of necessary FE simulations is ensured. Additionally, the utilized Model of Rubber Phenomenology (MORPH) is improved in view of the industrial use.

It is shown that the identification of material parameters using component-oriented specimens leads to a much better approximation of the original component behaviour than using standard specimens. Additionally, it is shown that the use of a material law which can consider characteritic properties of elastomers, is absolutely necessary.

\section{Keywords}

Parameter identification, shape optimization, shape identification, rubber materials, finite element method, material characterization, numerical integration 


\section{Einleitung}

In praktisch allen technischen Bereichen stehen Ingenieure heute vor der Herausforderung, Aussagen über Werkstoffe, Bauteile, Prozesse oder ganze Prozessketten zu treffen, deren Eigenschaften nur teilweise bekannt sind. Ihre Aufgabe besteht unter anderem darin, möglichst viele Informationen zu sammeln und auf Basis derer die unbekannten Eigenschaften direkt oder indirekt zu bestimmen. Beispielsweise können in der Biomechanik bei der nichtinvasiven Untersuchung eines Körperteils Informationen über Form, Beschaffenheit und Farbe der Oberfläche gewonnen werden, die tatsächliche Zusammensetzung des darunter liegenden Gewebes bleibt jedoch unbekannt. Gleiches gilt für die Betrachtung der stofflichen Eigenschaften von Bauteilen, welche üblicherweise durch geeignete Materialmodelle dargestellt werden. Sobald die Geometrie hinreichend komplex ist, kann die direkte Charakterisierung der Materialeigenschaften am Bauteil durch einfache Versuche nur sehr eingeschränkt realisiert werden. Die Weiterentwicklung von Verfahren zur Identifikation vorhandener, jedoch nicht sichtbarer Konturen innerhalb eines Bauteils sowie zur indirekten Ermittlung von Materialeigenschaften hat deshalb eine entscheidende Bedeutung für Industrie und Wissenschaft. Eine weitere Kernaufgabe des Ingenieurwesens besteht in der Entwicklung neuer bzw. in der Verbesserung vorhandener Produkte und Prozesse. Ein populäres Beispiel hierfür ist der Fahrzeugbau, bei welchem seit mehr als 100 Jahren eine stetige Weiterentwicklung stattgefunden hat und ein Ende derselben nicht zu erwarten ist. Im Fokus der Optimierungsprozesse stehen dabei sowohl die Fahrzeugeigenschaften als auch alle Aspekte des Herstellungsprozesses. Die Kombination von äußeren Faktoren wie politischen Vorgaben, Mitbewerbern am Markt und einem stetigen Preisdruck führt dazu, dass selbst kleine Baugruppen oder Bauteile bezüglich Gewicht, Form, Haltbarkeit, Produktionskosten, Wiederverwertbarkeit u.v.m. ständig optimiert werden. Im gleichen Maße sind auch die Zulieferer der Fahrzeugindustrie gezwungen, wiederkehrend ihre Fertigungsprozesse zu überarbeiten und zu optimieren, um z.B. die Haltbarkeit der Umformwerkzeuge zu verbessern, Fertigungszeiten zu verringern oder die Energiekosten zu senken.

Aus diesem Grund existiert heute eine ganze Reihe verschiedener mathematischer Verfahren zur Bearbeitung der vorbenannten Aufgaben. Seit dem Einzug der Rechentechnik in Forschung und Entwicklung wurden viele dieser Ansätze auf sehr unterschiedliche Weise und mit stark variierendem Umfang in Software-Produkte implementiert. Ergänzt wird dies durch das Einbinden numerischer Simulationsverfahren, welche auf der Methode der finiten Elemente (FEM) basieren. Infolge dessen gehört die Möglichkeit zur Identifikation von Materialparametern sowie die Optimierung von Bauteilen hinsichtlich unterschiedlicher Kriterien unter Einsatz kommerzieller Software bereits zum Stand der Technik.

Dennoch existieren nach wie vor in mehreren Bereichen Verbesserungspotentiale. Dies gilt insbesondere, wenn mehrere Aspekte der Identifikation oder Optimierung gleichzeitig hohen Anforderungen genügen müssen. Ein Beispiel dafür stellt die Materialmodellierung dar. 
Zwar kann das Verhalten metallischer Wertstoffe in FEM-Simulationen dargestellt werden, jedoch sind moderne Stoffgesetze, welche z.B. neben der isotropen auch die kinematische und formative Verfestigung von Metallen gut darstellen können, in kommerzieller Software nur unzureichend implementiert. Gleiches gilt für Elastomere, deren typisch inelastisches Verhalten bzw. Eigenschaften wie der Mullins-Effekt oder Hystereseerscheinungen ebenfalls nicht genügend durch die vorhandenen Stoffgesetze der FEM-Programme abgebildet wird. Auch wenn oft die Möglichkeit zur Implementierung eigener Materialmodelle besteht, ist die Identifikation der zugehörigen Parameter mit programmeigenen Routinen sehr aufwändig oder gar nicht möglich. Erschwert wird dies zusätzlich, wenn der Anwender ein bestimmtes numerisches Verfahren zur Identifikation nutzen bzw. beeinflussen möchte oder einen spezifischen Umgang mit den Messdaten benötigt.

Gerade die Verfügbarkeit und vor allem die gezielte Steuerung der notwendigen Optimierungsverfahren ist nur zum Teil zufriedenstellend. So erfordern die angewandten Algorithmen häufig eine große Anzahl von Optimierungsschritten, was sich vor allem dann sehr negativ auf die benötigte Rechenzeit auswirkt, wenn die im Rahmen der Identifikation oder Optimierung benötigten FEM-Simulationen sehr zeitaufwändig sind. Die automatische Adaption der Verfahrenseigenschaften an die jeweilige Aufgabe zur Minimierung der Optimierungsschritte und die Sicherstellung eines robusten Verhaltens auch für schlecht konditionierte Probleme stellt daher ein wichtiges Betätigungsfeld dar.

Eine Herausforderung ist auch die Integration hauseigener Programme in bereits existierende Software zur Optimierung oder Identifikation, welche oft mit Einbußen bezüglich Funktionalität und Effizienz verbunden ist. Kern der vorliegenden Arbeit ist deshalb die Entwicklung der Optimierungssoftware SPC-Opt (Kap. 2), mit welcher die Geometrie technischer Bauteile optimiert und Materialparameter zur Charakterisierung von Materialeigenschaften sowie Formparameter zur Beschreibung geometrischer Bauteileigenschaften identifiziert werden können. Die gezielte Steuerung der implementierten Optimierungsverfahren ist dabei ebenso wichtig wie die Entwicklung eines robusten, aber dennoch effizienten Verfahrens zur Identifikation von Parametern. Ein weiterer Schwerpunkt liegt bei der Konzeption einer modularen Schnittstelle zur Einbindung hauseigener und kommerzieller FEM-Software. Nur so ist es möglich, ein breites Anwendungsfeld zu ermöglichen und bei Bedarf zu erweitern. Auch die Nutzung paralleler Rechenarchitekturen soll möglich sein, da diese in Form von verteilten Netzwerken und Rechenclustern in vielen Firmen und wissenschaftlichen Einrichtungen zur Verfügung stehen.

Um eine möglichst vielfältige Auswahl von Aufgaben aus den Bereichen der Formoptimierung sowie Parameteridentifikation bearbeiten zu können, wird eine Reihe dafür geeigneter mathematischer Methoden in die Software integriert. Dies betrifft bezüglich der Parameteridentifikation vor allem das in Kap. 3 beschriebene Levenberg-Marquardt-Verfahren, die zugehörige Fletcher-Erweiterung und eine Reihe von Maßnahmen zur Verbesserung der Robustheit des Verfahrens. In Kap. 4 werden verschiedene Ansätze vorgestellt, um auch Formoptimierungen durchführen zu können. Dabei werden auch Möglichkeiten zur Approximation der Hesse-Matrix betrachtet. Den Abschluss dieser Thematik bilden die Verfahren zur Startparametervariation zur Auffindung globaler Minima, auf welche in Kap. 5 eingegangen wird. 
In Kooperation mit verschiedenen Unternehmen aus dem Bereich der Gummiindustrie wurde im Rahmen eines industriefinanzierten Projektes die Optimierungssoftware SPC-Opt in Form einer Programmauskopplung derart weiterentwickelt, dass sie auch im industriellen Umfeld gut nutzbar ist. Den Anstoß zu diesem Projekt gab die Tatsache, dass die Identifikation von Materialparametern in der Industrie zwar zum Stand der Technik gehört, dabei jedoch speziell für die in der Gummiindustrie verwendeten Elastomere gleich mehrere Herausforderungen existieren. Diese betreffen zum einen die Verfügbarkeit inelastischer Materialgesetze zur korrekten Abbildung des Materialverhaltens in den kommerziellen FEM-Programmen sowie die korrekte Durchführung der für die Identifikation notwendigen Experimente und der zugehörigen Messdatenaufbereitung. Zum anderen besteht bei Elastomeren das Problem, dass deren Materialeigenschaften nicht nur von der chemischen Zusammensetzung, sondern auch vom Herstellungsprozess und der Geometrie abhängen. Dies ist für die Identifikation nachteilig, da die Stoffparameter gewöhnlich an Versuche mit Standardprobekörpern angepasst werden und sich diese geometrisch und herstellungstechnisch stark von den Bauteilen unterscheiden. In Kap. 6 wird anhand eines konkreten Bauteils dargelegt, wie diese Probleme bewältigt werden können. Der Schwerpunkt liegt dabei bei der Identifikation anhand sogenannter bauteilnaher Probekörper sowie der Verwendung des inelastischen Stoffgesetzes "MorPH" zur Abbildung des Materialverhaltens. 


\section{Das Programm SPC-Opt}

Sowohl für die Identifikation von Parametern als auch zur Lösung von Problemen der Strukturoptimierung existiert eine große Menge kommerzieller und freier Software. So beinhalten die weit verbreiteten FEM-Programme $A B A Q U^{*}$ oder $M S C . M A R C^{*}$ bereits seit mehreren Jahren Module zur Anpassung enthaltener Stoffgesetze an homogene Standardversuche. Seitdem wurden diese Funktionalitäten weiterentwickelt und erweitert. In jüngerer Zeit erfolgte zudem die Implementierung von Modulen zur Form- bzw. Topologieoptimierung, wenn auch auf unterschiedliche Art und Weise.

Andere kommerzielle Programme, welche meist keine nativen FEM-Solver beinhalten, wurden speziell im Hinblick auf die Bearbeitung von Optimierungsaufgaben entwickelt. Bekannte Vertreter sind zum Beispiel TOSCA ${ }^{*}$, LS-OPT $^{*}$, OptiStruct $^{*}$ sowie FT-Optimization ${ }^{*}$. Diese stellen dem Anwender meist eine deutlich umfangreichere Palette von Werkzeugen zur Verfügung. Mangels eigener FEM-Solver steht in den Programmen eine steigende Anzahl Schnittstellen zum Einbinden externer FEM-Software zur Verfügung. Die Nutzung von FEMProgrammen ist hierbei keineswegs zwingend, da für eine ganze Reihe Optimierungsaufgaben die notwendigen Funktionsauswertungen auch in anderer Form, z.b. analytisch, ermittelt werden können. In diesem Kontext ist auch die mathematische Umgebung $\overline{M A T L A B}^{{ }^{*}}$ zu nennen, welche zur effizienten Nutzung zwar Programmierfähigkeiten seitens des Nutzers erfordert, im Gegenzug jedoch aufgrund ihrer historischen Entwicklung eine hohe Flexibilität und ein breites Spektrum effizienter Lösungsalgorithmen bereit stellt. Ursprünglich als Konstruktionswerkzeuge entwickelt, bieten moderne CAD-Systeme die Möglichkeit, bereits im Zuge der Modellerstellung Topologie- oder Formoptimierungen durchzuführen. Der große Vorteil aus Sicht des Nutzers ist die Tatsache, dass nach einer solchen Optimierung das aktualisierte Modell ohne zusätzlichen Aufwand in Form der ursprünglichen CAD-Beschreibung vorliegt. Beispiele hierfür sind NX $^{*}$, SOLIDWORKS ${ }^{*}$ oder $P T C / C r e{ }^{*}$. Da die CAD-Parameter direkt als Designvariablen genutzt werden, wird das FE-Netz im Gegensatz zur Optimierung mit den oben genannten FEM-Programmen nach jeder Formänderung neu erstellt.

Neben kommerzieller Software gibt es auch eine große Zahl freier Programme, welche häufig im Verlauf von Forschungsprojekten an oder in Zusammenarbeit mit universitären Einrichtungen entstanden sind. In den meisten Fällen sind diese auf bestimmte Anwendungsbereiche spezialisiert und besitzen diesbezüglich eine hohe Kompetenz bzw. einen Entwicklungsvorsprung gegenüber alternativer Software. Damit geht jedoch auch oft eine Einschränkung der Einsatzmöglichkeiten einher. Naturgemäß unterscheiden sich diese Programme in ihrem Funktionsumfang und der Bedienerfreundlichkeit sehr stark voneinander. Vertreter dieser Art sind u.a. $A L G L I B{ }^{*}$ und $A D O L-C^{*}$, wobei $A D O L-C^{*}$ zur umfangreichen Bibliothek COIN-OR gehört, welche auch andere Werkzeuge zur Optimierung beinhaltet. Falls

\footnotetext{
*Detaillierte Angaben sind im Anhang A.1 aufgeführt.
} 
Möglichkeiten zur stetigen Weiterentwicklung vorhanden sind und genutzt werden, ist auch der Übergang zu einem kommerziellen Produkt, wie die Entwicklung von $C A R A T++*$ zeigt, nicht ungewöhnlich.

Auch die in diesem Kapitel vorgestellte Optimierungssoftware SPC-Opt (Scientific Parallel Computing and Optimization) wurde entsprechend den Erfordernissen verschiedener Forschungsprojekte realisiert. Den Ausgangspunkt für die Programmentwicklung bildete die Erarbeitung neuer Beschreibungsweisen des elastisch-plastischen Verhaltens metallischer Werkstoffe (Bucher, 2001; Grewolls, 1998; Lindner und Kreißig, 2004; Panhans, 2006) und die in diesem Kontext an der TU-Chemnitz entstandene FEM-Forschungssoftware SPC-PM2AdN ${ }^{*}$. Im Zusammenhang mit der dadurch notwendigen Parameteridentifikation sowie Untersuchungen zur Auswertung von inhomogenen Verschiebungsfeldern (Benedix, 2000; Bohnsack, 1997; Kreißig et al., 2007) wurde die Funktionalität des Programmes nochmals deutlich erweitert, u.a. durch die Implementierung des bewährten Levenberg-Marquardt-Verfahrens (vergl. auch Kap. 3.6). Aufgrund der Entwicklungshistorie wies das in FORTRAN geschriebene $S P C-P M 2 A d N l$ jedoch mehrere strukturelle Nachteile auf, was eine effiziente Weiterentwicklung auf der vorhandenen Programmbasis zunehmend erschwerte.

\subsection{Anforderungsprofil und Funktionalität von $S P C-O p t$}

Im Rahmen des DFG-Projektes "Optimierung von Werkzeugen und Prozessparametern bei Massivumformverfahren" (Schellenberg et al., 2007; Schellenberg und Kreißig, 2009; Schellenberg et al., 2010) ergaben sich mehrere Kernforderungen an die verwendete Optimierungssoftware. Neben der Bereitstellung der Funktionalität zur Materialparameteridentifikation des bisher genutzten Programmes SPC-PM2AdNl sollte nunmehr auch die Bearbeitung verschiedener Aufgabenstellungen aus dem Bereich der Formoptimierung möglich sein. Zudem wurde es notwendig, neben hauseigenen FEM-Programmen auch kommerzielle Software einbinden zu können. Da in diesem Zusammenhang ein hoher Rechenaufwand für die notwendigen FEM-Rechnungen zu erwarten war, empfahl sich darüber hinaus die Nutzung paralleler Rechentechniken. Die Entscheidung fiel deshalb zugunsten einer neuen Optimierungssoftware in $\mathrm{C}++$, wobei im Hinblick auf künftige Erweiterungen folgende Paradigmen berücksichtigt wurden:

- Modularisierung des Programms sowie Nutzung von Templates für gleichartige Strukturen (z.B. FEM-Schnittstellen)

- Verwendung des MPI-Standards zur Parallelisierung

- Allgemeine Definition einer FEM-Schnittstelle zur flexiblen Nutzung verschiedener FEM-Programme

- Automatische Erstellung von Log-Dateien in allen Programmteilen zur Sicherstellung einer effizienten Fehlerbearbeitung

${ }^{*}$ Detaillierte Angaben sind im Anhang [A.1] aufgeführt. 
- Nutzung des Versionskontrollsystems $\widehat{S V N}^{*}$ zur Versionsverwaltung und Koordination der Mitarbeit verschiedener Personen

- Software-Dokumentation mit Hilfe von DOXYGEN

- Nutzung plattformunabhängiger Programmierwerkzeuge, z.B. $C M A K E^{*}$ und $\mathrm{POCO}^{*}$

- Strukturierung der Ein- und Ausgabedaten im XML-Format

Im Ergebnis dessen liegt nunmehr ein Programmpaket vor, mit welchem verschiedene Aufgaben auf den Gebieten der Materialparameteridentifikation, Parameterstudien, Formidentifikation und Formoptimierung erfolgreich bearbeitet werden können. Zwar ist $S P C-O p t$ grundsätzlich für Multiprozessorsysteme konzipiert, kann jedoch ebenso in herkömmlichen heterogenen Computernetzwerken oder auf einzelnen Computern genutzt werden.

\subsection{Programmstruktur}

Das Programmpaket besteht aus drei Komponenten, wobei die eigentliche Identifikation bzw. Optimierung mit dem Hauptprogramm "SPC-Opt" erfolgt. Des Weiteren steht für die Aufbereitung von Messdaten das Modul "ExpFileGenerator" zur Verfügung. Die Konvertierung von FEM-Eingabedaten in ein programmeigenes Format erfolgt mit dem "Converter", einer Auskopplung vom Hauptprogramm, welche lediglich die FEM-Schnittstellen beinhaltet.

Die zentrale Struktur von SPC-Opt stellt der Programmrumpf mit den Basisklassen und notwendigen Grundfunktionen dar. Diese beziehen sich in erster Linie auf die Steuerung der Optimierung sowie die Verwaltung der zugehörigen FEM-Daten. Als Grundlage hierfür dienen verschiedene Werkzeuge, unter anderem

- eine Klasse zum systemunabhängigen Lesen und Schreiben von Dateien,

- ein XML-Parser zur Vereinheitlichung des Datenformats in XML

- sowie ein komfortables System zum automatischen Anlegen von Log-Dateien.

Durch die Implementierung der mathematischen Skriptsprache LUA (Ierusalimschy, 2006) können durch den Anwender auch mathematische Ausdrücke bzw. Gleichungen eingegeben und an verschiedenen Stellen innerhalb von $S P C$-Opt genutzt werden.

Neben dem Programmrumpf existiert eine Reihe unterschiedlicher Module, mit deren Hilfe die verschiedenen Aspekte von Optimierungsproblemen bearbeitet werden können. Diese werden in den nachfolgenden Kap. 2.3-2.6 näher betrachtet und sind in Bild 2.1] schematisch dargestellt. Hierbei werden die obligatorischen Module mit durchgehenden Verbindungslinien gekennzeichnet, wohingegen unterbrochene Linien für die Anbindung der optionalen Module genutzt werden.

\footnotetext{
*Detaillierte Angaben sind im Anhang A.1 aufgeführt.
} 


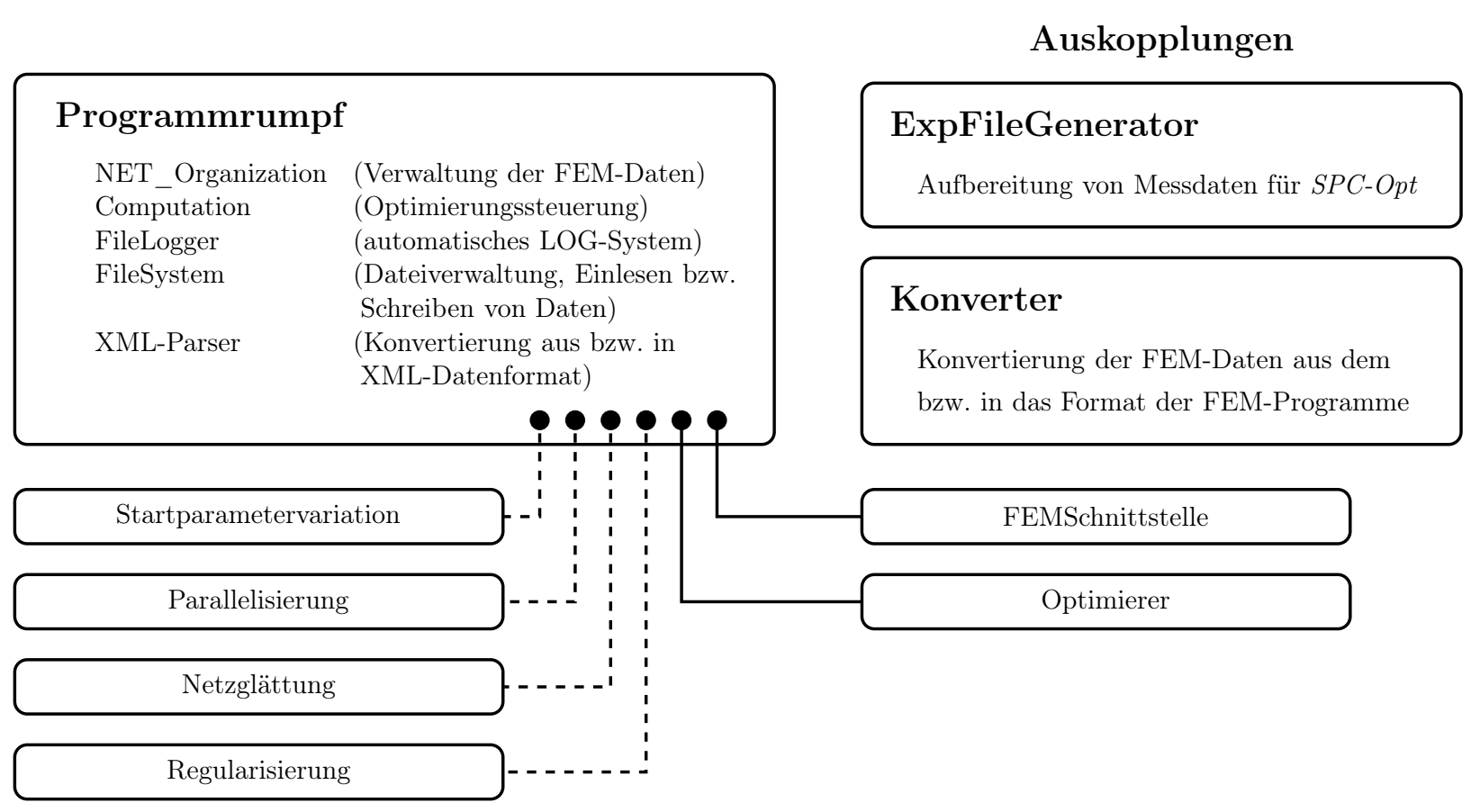

Bild 2.1: Struktureller Aufbau von SPC-Opt.

Für Identifikationsaufgaben steht zur Aufbereitung von Messdaten aus Experimenten das Modul "ExpFileGenerator " zur Verfügung. Es können hierbei rampenartige sowie stationäre und instationäre zyklische Belastungen verarbeitet werden. Die Art der Belastung ist dafür unerheblich (soweit sie in SPC-Opt implementiert ist) und kann z.B. in Form von Verschiebungen, Kräften oder Spannungen vorliegen. Nach der Aufbereitung der Daten mit Hilfe des ExpFileGenerators steht die gesamte Belastungsgeschichte des Versuchs in einer ".exp" -Datei zwecks späterer Verwendung in $S P C$-Opt zur Verfügung.

Zur Erstellung der FEM-Modelle sowie für die Durchführung der notwendigen Simulationen können hauseigene FEM-Programme oder kommerzielle Software-Pakete wie $A B A Q U S *$ oder MSC.MARC ${ }^{*}$ genutzt werden. Die in SPC-Opt enthaltenen Module der verschiedenen FEM-Schnittstellen gestatten es, die Eingabedateien der unterstützten FEM-Programme zu parsen und in einem programmeigenen "sim" -Format mit der Möglichkeit zur anschließenden Modifikation auszugeben. In dieser Datei werden zudem die für das jeweilige Experiment gültigen Zielfunktionsanteile formuliert. Um eine hohe Flexibilität und Erweiterbarkeit zu garantieren, wird als Datenformat der XML-Standard verwendet. Bei Identifikationsaufgaben wird demnach meist für jedes Experiment eine ".sim" -Datei und eine gleichnamige ".exp" Datei generiert, bei Optimierungen ohne Messdaten genügt die ".sim" -Datei.

Die Steuerung der Identifikation bzw. Optimierung erfolgt mit Hilfe einer übergeordneten "opt"-Datei, welche ebenfalls im XML-Standard gehalten ist. Diese beinhaltet die Verwaltung der einzelnen Experimente, die Liste der zu identifizierenden Materialparameter mit den entsprechenden Verweisen sowie einige globale Einstellungen. Zudem können hier bei Bedarf auch die Steuergrößen des verwendeten Optimierungsverfahrens modifiziert werden.

*Detaillierte Angaben sind im Anhang $[$ A.1 aufgeführt. 
Im Folgenden wird die Funktionalität der wichtigsten Programmbestandteile erläutert. Hierbei ist darauf hinzuweisen, dass die Entwicklung des Programmpakets nicht ausschließlich durch den Verfasser der vorliegenden Arbeit erfolgte. Als Grundlage für die Implementierung der einzelnen Module dienten auch vom Verfasser betreute studentische Arbeiten, in welchen die existierenden Algorithmen der jeweiligen Funktionalität im Einzelnen betrachtet und auf Eignung zur Bearbeitung praktischer Problemstellungen untersucht wurden. In programmiertechnischer Hinsicht ist zudem die Mitarbeit von Wulf (Wulf et al., 2012, 2013) zu nennen, welcher eine große Hilfe bei notwendigen Umstrukturierungen und Programmerweiterungen war. Zum besseren Verständnis beschränken sich die nachfolgenden Ausführungen zunächst auf die programmiertechnischen Aspekte des Programmes. Die Darstellung der implementierten mathematischen Algorithmen erfolgt gesondert in Kap. 3 bis Kap. 5 .

\subsection{Optimierer}

Welcher Algorithmus sich zur Lösung eines Optimierungsproblems eignet, hängt stark von der Aufgabenstellung sowie den Eigenschaften der Zielfunktion ab. SPC-Opt wurde entwickelt, um Aufgaben aus den Bereichen der Parameteridentifikation (Kap. 3) sowie der Formoptimierung (Kap. 4) effizient bearbeiten zu können. Für die Klasse der Identifkationsprobleme liegt der Anwendungsschwerpunkt bei der Anpassung von Materialparametern an vorhandene Experimente, da dies auch im industriellen Umfeld eine wiederkehrende Herausforderung darstellt (Kap. 6). Dies impliziert jedoch keine Beschränkung auf Probleme der Materialparameteridentifkation - auch die Anpassung von Formparametern ist in vielen Bereichen von praktischer Bedeutung.

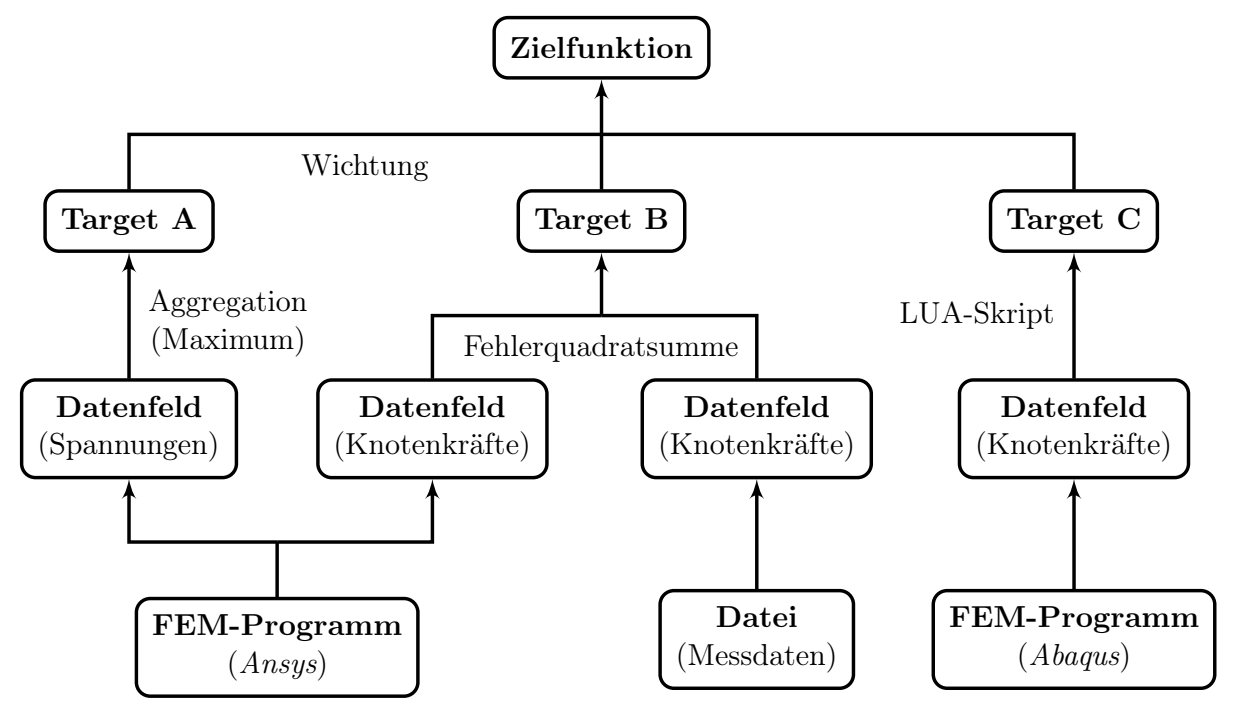

Bild 2.2: Beispiel für den Aufbau der Zielfunktion.

Allen vorgenannten Problemstellungen ist gemein, dass eine problembezogene Zielfunktion entsprechend Gl. 3.4 gebildet und anschließend minimiert wird. Um dem Nutzer ein 
möglichst flexibles und dennoch intuitives System zur Definition der Zielfunktion zur Verfügung zu stellen, beinhaltet SPC-Opt ein Baukastensystem zur entsprechenden Definition. Der Aufbau einer Zielfunktion ist beispielhaft in Bild 2.2 dargestellt. Durch den Nutzer können verschiedene Anteile ("Targets") definiert werden, aus welchen sich die zu minimierende Größe konstituiert. Die einzelnen Targets wiederum basieren auf FEM-Ergebnissen, Messdaten oder anderen Targets und können mit mathematischen Operationen modifiziert werden. Anhand des Aufbaus der Zielfunktion kann festgestellt werden, ob es sich um ein Fehlerquadratminimum-Problem (FQM) handelt, was üblicher Weise bei Identifikationssaufgaben der Fall ist. Der Optimierer kann somit auf das in Kap. 3.6 dargestellte LevenbergMarquardt-Fletcher-Verfahren (LMF) zurückgreifen. Trifft dies jedoch nicht zu, z.B. bei Problemen der Formoptimierung, eignen sich die in Kap. 4.1 angegebenen Trust-Region bzw. Line-Search-Verfahren zur Lösung. Dabei muss jedoch beachtet werden, dass die HesseMatrix nicht mehr mit guter Näherung auf Basis der Ableitungen bestimmt werden kann, sondern durch eines der in Kap. 4.2 angegebenen Verfahren (BFGS, SR1, ...) approximiert werden muss. Falls das LMF-Verfahren verwendet wird, kann der Optimierer zusätzlich entsprechend Kap. 3.7 Restriktionen in Form von Boxbeschränkungen in die Lösung des Minimierungsproblems einbeziehen. Abschließend werden dem Optimierungsmodul noch die analytisch oder auf Basis der Differenzenquotienten numerisch ermittelten Ableitungen zur Verfügung gestellt, welches somit einen neuen Parametersatz berechnet und zurückgibt. Der beschriebene Ablauf wird in Bild 2.3 verdeutlicht.

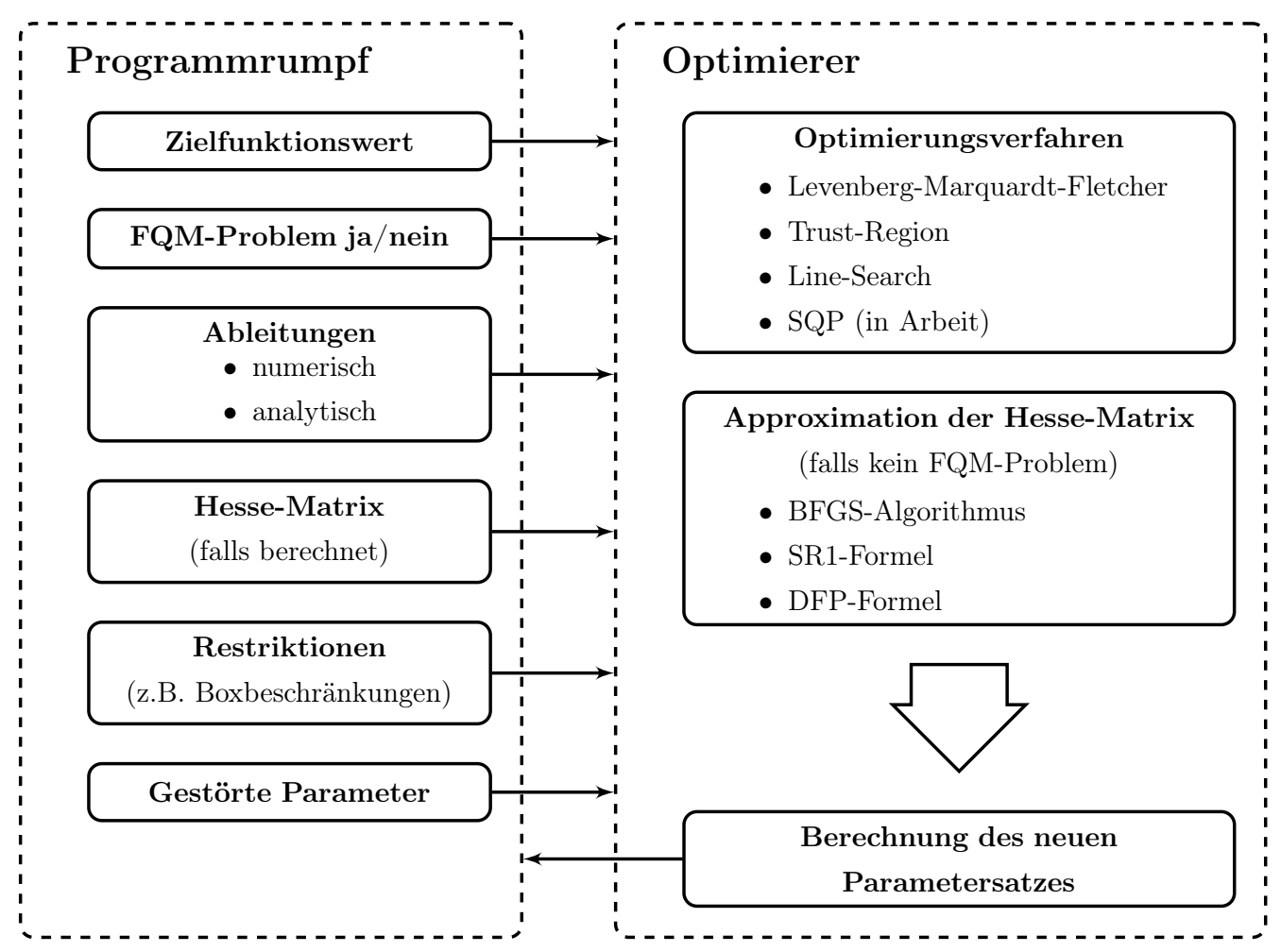

Bild 2.3: Aufbau des Optimierers und Datenfluss. 


\subsection{FEM-Schnittstellen}

Für die Bestimmung des Zielfunktionswertes ist es notwendig, darin enthaltene Größen für vorhandene bzw. gestörte Paramtersätze zu berechnen. In den meisten Fällen werden hierfür kommerzielle oder hauseigene FEM-Programme genutzt, jedoch ist auch die Verwendung mathematischer Software (z.B. MATLAB*) oder Skriptsprachen (z.B. LUA) üblich. Der Einfachheit halber werden die verschiedenen Programmtypen im Weiteren unter dem Begriff "FEM-Programme" zusammengefasst. Voraussetzung für deren Nutzung ist die Implementierung entsprechender Schnittstellen in SPC-Opt, wobei der programmiertechnische Aufwand sehr unterschiedlich ist. Die Funktionalität einer Schnittstelle kann entsprechend ihrer Aufgaben unterteilt werden - Parsen der FEM-Eingabedatei, Schreiben der Eingabedatei zur Simulation und Einlesen der daraus resultierenden Ergebnisse.

Vor Start der Optimierung ist das Einlesen und Aufbereiten einer FEM-Eingabedatei notwendig. Die grundsätzliche Vorgehensweise besteht darin, dass ein an das jeweilige FEMProgramm angepasster Parser die Eingabedatei durchläuft, alle für $S P C$-Opt relevanten Daten einliest und in einem programmeigenen Format speichert. Innerhalb der Eingabedatei werden die Abschnitte der tatsächlich eingelesenen Daten durch entsprechende Platzhalter ersetzt. Das Resultat stellt ein Template der originalen Eingabedatei dar, in welcher sich nur noch die Platzhalter für die eingelesenen Daten sowie die Textblöcke der nicht berücksichtigten Bereiche befinden. Die auf dieser Basis generierte ".sim" -Eingabedatei enthält die geparsten Informationen bezüglich FE-Knoten, FE-Elementen, Materialien, Randbedingungen u.a., sowie zusätzlich das oben beschriebene Eingabedatei-Template.

Durch den Anwender müssen anschließend noch die Informationen zur Zielfunktion ergänzt werden. Um diese erfolgreich definieren zu können, existieren zwei synthetische Hilfsstrukturen in Form von ID-Listen und Step-Listen. Zum einen sind die Ortsabgaben bezüglich der Messstellen und damit den korrespondierenden FE-Knoten notwendig. Mit Hilfe der ID-Listen können deshalb ein oder mehrere Knoten bzw. Elemente gruppiert und an geeigneter Stelle bei Bildung der Zielfunktion verwendet werden. Zum anderen können mit Hilfe von Step-Listen Zeitpunkte festgelegt werden, zu denen ein Messwert oder eine Ausgabegröße benötigt wird. Die Projektion der Eingangsdaten auf die einzelnen Lastschritte obliegt hierbei dem Anwender. Allerdings wird bei der Aufbereitung von Messdaten bereits die entsprechende Step-Liste erzeugt und kann an dieser Stelle verwendet werden.

Während der eigentlichen Optimierung hat die FEM-Schnittstelle zwei Funktionen. So muss zu Beginn eines jeden Optimierungsschrittes dem aktuellen bzw. den gestörten Parametersätzen entsprechend die jeweilige FEM-Eingabedatei aktualisiert und geschrieben werden. Bei einigen kommerziellen Programmen ist dies jedoch nicht unproblematisch, insbesondere wenn Materialparameter für nutzereigene Materialmodelle identifiziert werden sollen. Dies liegt daran, dass bei der Verwendung der Nutzschnittstelle für Materialmodelle die Parameter teilweise direkt im Quellcode hinterlegt sind und nicht ohne Weiteres innerhalb der FEM-Eingabedatei modifiziert werden können.

Nach dem erfolgreichen Abschluss einer Simulation werden die benötigten Größen aus den FEM-Ausgabedateien extrahiert, welche im Klartext vorliegen müssen. Sind die Informatio-

\footnotetext{
*Detaillierte Angaben sind im Anhang A.1 aufgeführt.
} 
nen bezüglich der Mess- und Zeitpunkte vollständig, kann auch die Zielfunktion ausgewertet werden.

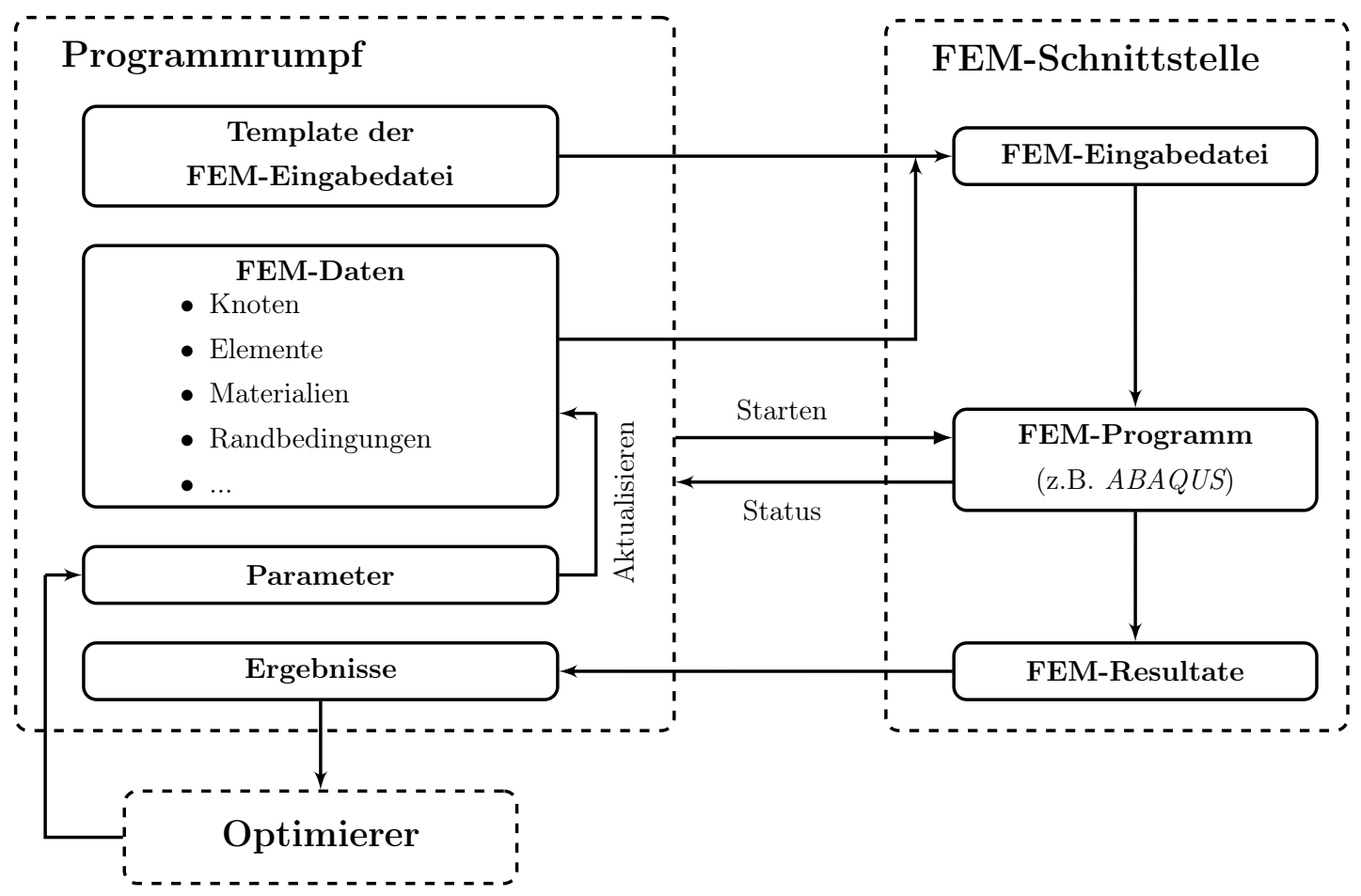

Bild 2.4: Grundsätzliche Funktionsweise einer FEM-Schnittstelle.

In Bild 2.4 wird der beschriebene Ablauf schematisch dargestellt und verdeutlicht die klare Abgrenzung der FEM-Schnittstelle vom Programmrumpf. Möglich ist dies durch die Nutzung der Fabrikmethode, einem Entwurfsmuster aus der Softwareentwicklung, welche z.B. in (Gamma et al., 1994) beschrieben wird. Das Anlegen der FEM-Eingabedateien, der Start des FEM-Programms und das Auswerten der Resultate werden durch den Programmrumpf zwar gesteuert, die eigentliche Funktionalität (welche sich in Abhängigkeit des Programms in Umfang und Komplexität stark unterschieden kann) befindet sich jedoch komplett in der FEM-Schnittstelle. Es ist somit auch unproblematisch, dass erst während der Laufzeit bekannt wird, welche FEM-Programme bzw. wie viele Instanzen von diesen benötigt werden. Zudem gestattet diese Programmierweise die Ergänzung weiterer FEM-Schnittstellen, ohne den Programmrumpf modifizieren zu müssen. Die Vorgehensweise wird deshalb in vergleichbarer Form auch an anderen Stellen von SPC-Opt angewendet, z.B. bei der Implementierung des Optimierers oder der Startparametervariation. Zum aktuellen Zeitpunkt stehen in $S P C$-Opt die nachfolgenden FEM-Schnittstellen zur Verfügung:

- $A B A Q U^{*}$,

- $A N S Y S^{*}$

- MSC.MARC*,

${ }^{*}$ Detaillierte Angaben sind im Anhang [A.1] aufgeführt. 
- das hauseigene FEM-Programm SPC-PM2AdNl und darauf basierende Weiterentwicklungen sowie,

- LUA-Skripte.

Da die Schnittstellen der kommerziellen FEM-Programmen deutlich komplexer als die der hauseigenen Programme bzw. LUA-Skripte sind, wird deren Funktionsweise im Folgenden kurz dargestellt.

\section{FEM-Schnittstelle $A B A Q U S$}

Als übliches Eingabeformat für $A B A Q U S$ wird die ".inp" -Datei genutzt, welches auch durch Preprocessoren anderer Anbieter, z.B. HYPERMESH* oder PATRAN ${ }^{*}$, unterstützt wird. Das Einlesen der FEM-Daten ist infolge der guten Strukturierung des Eingabeformates problemlos möglich. Zudem ist der Aufwand für den Nutzer, die zur Bildung der Zielfunktion benötigten Größen zu definieren sowie deren Ausgabe zeitlich und örtlich festzulegen, gering. Geometrische Eigenschaften des Modells können allerdings nicht in der Eingabedatei hinterlegt werden, was bei der Bearbeitung von Aufgaben der Formidentifikation bzw. -optimierung von Bedeutung ist. Einen Ausweg stellt die Nutzung von Referenzpunkten dar, welche wie FE-Knoten behandelt werden und deshalb auch in der ".inp" -Datei vorhanden sind. Darüber hinaus bietet sich die Definition von Variablen in $A B A Q U S$ an, welche ebenso in der Eingabedatei enthalten sind. Das FE-Netz sollte bei dieser Vorgehensweise parametrisiert werden und von den definierten Variablen abhängig sein. Zur eigentlichen Netzerstellung werden durch ABAQUS unterstützte PYTHON-Skripte verwendet.

Das native ".odb"-Ausgabeformat steht nicht als Klartext zur Verfügung, kann jedoch nach der Simulation durch einen nochmaligen Aufruf von $A B A Q U S$ in Kombination mit einem entsprechenden PYTHON-Skript (welches automatisch von SPC-Opt angelegt wird) geparst werden. Die auf dieser Basis erzeugte ".aba"-Datei enthält somit alle durch SPC-Opt angeforderten Ergebnisse und kann problemlos eingelesen werden.

\section{FEM-Schnittstelle $A N S Y S$}

Auch bei $A N S Y S$ gibt es die Möglichkeit, innerhalb des Preprocessors die FEM-Daten als ".inp"-Eingabedatei im Klartext abzulegen. Allerdings ist der Transfer von Informationen bezüglich der Geometrie noch schwieriger als bei ABAQUS. Als Grundlage dient deshalb das ".cdb" -Format, welches zwar nicht als Eingabedatei für den Solver verwendet werden kann, jedoch im Gegenzug alle geometrischen Eigenschaften des Modells enthält. Mit Hilfe dieser Informationen sowie notwendigen Anpassungen durch den Anwender ist die ANSYSSchnittstelle in der Lage, eine entsprechende ".inp" -Datei für die Simulation zu erzeugen.

Die Ausgabe der Ergebnisse ist demgegenüber einfach, da in der Eingabedatei Art, Zeitpunkt und Position der auszuwertenden Zielgrößen definiert und das Ausgabeformat festgelegt werden können. Im Anschluss an die Simulation können die benötigten Ergebnisse deshalb problemlos aus der angelegten ".rst" -Datei eingelesen werden.

\footnotetext{
*Detaillierte Angaben sind im Anhang A.1 aufgeführt.
} 
FEM-Schnittstelle $M S C . M A R C$

Zwar besitzt auch dieses FEM-Programm eine Eingabedatei (".dat")im Klartextformat, jedoch müssen bei der Implementierung der FEM-Schnittstelle zwei Gesichtspunkte von $M S C . M A R C$ berücksichtigt werden:

- Die Ausgabesteuerung von Zielgrößen ist mit Hilfe der Eingabedatei nur bedingt nutzbar. Das native ".t19" -Format ist nicht nur unübersichtlich, sondern kann auch sehr groß werden, da teilweise erheblich mehr als die durch den Anwender gewünschten Daten gespeichert werden. Auch bei der alternativen Ausgabe in einer der im Rahmen der Simulation angelegten Dateien (z.B. ".sts" -Datei) ist die Ausgabestruktur der verschiedenen Größen unübersichtlich und unterscheidet sich zudem für praktisch alle Ausgabetypen voneinander. Noch schwerwiegender ist jedoch, dass bei beiden Varianten die Genauigkeit der geschriebenen Werte auf fünf bis sieben Stellen beschränkt ist. Werden auf dieser Basis die Zielfunktionswerte und Gradienten berechnet, wirken sich diese Ungenauigkeiten zwangsläufig negativ auf die Optimierung aus.

- Für die Verwendung eigener Stoffgesetze ist in MSC.MARC die Nutzerschnittstelle HYPELA2 vorgesehen. Eine direkte Steuerung der zugehörigen Materialparameter über die Eingabedatei ist in nativer Form jedoch programmseitig nicht angedacht. Vielmehr werden in den meisten Fällen die aktuellen Parameter direkt in den FORTRAN-Quellcode der Schnittstelle einprogrammiert. Dadurch muss für jeden Parametersatz der Solver $M A R C$ neu kompiliert werden, was vor allem im Hinblick auf eine Parallelisierung der FEM-Simulationen zu einem hohen zeitlichen Mehraufwand führt. Zudem treten potentiell Konflikte auf, falls verschiedene Instanzen von SPC-Opt gleichzeitig versuchen, das FEM-Programm zu kompilieren.

Eine praktikable Lösung für das Ausgabeproblem stellt das direkte Schreiben der Zielgrößen innerhalb der zur Verfügung stehenden User-Subroutinen von MSC.MARC dar. Damit kann nicht nur die Genauigkeit auf double precision erhöht, sondern auch die Ausgabe der Zielgrößen bezüglich Zeitpunkt und Ort dezidiert gesteuert werden. Dies erfolgt, indem für jede ".dat"-Eingabedatei eine zusätzliche ".cfg" -Datei mit den entsprechenden Informationen bezüglich der Ausgabegrößen erstellt wird. Nach dem Start des MARC-Solvers und dem Einlesen der FEM-Daten wird unmittelbar vor Beginn der Simulation diese Datei eingelesen und innerhalb der User-Subroutinen eine entsprechende Datenstruktur für die benötigten Zielgrößen angelegt. Nach jedem Lösungsinkrement werden alle bis dahin gespeicherten Ausgabedaten in eine ".mrc" -Datei geschrieben und stehen somit nach dem Abschluss der Simulation $S P C$-Opt zur Verfügung.

Wie aus der schematischen Darstellung des Informationsflusses in Bild 2.5 hervorgeht, werden bei der Nutzung von Parallelisierung durch MSC.MARC mehrere ".mrc"-Ausgabedateien angelegt. Der Grund hierfür ist, dass für die effiziente Parallelisierung das FE-Netz intern in verschiedene Bereiche (Domains) aufgeteilt wird und für jedes dieser Gebiete voneinander unabhängige Prozesse gestartet werden, innerhalb derer nur die Ergebnisse der jeweils enthaltenen Knoten und Elemente verfügbar sind. Beim Schreiben der Ergebnisse wird deshalb 
eine zusätzliche ".cpu"-Datei mit der Anzahl der beteiligten Rechenknoten angelegt. Anhand dieser Information kann in der Schnittstelle die Menge der angelegten Ergebnisdateien ermittelt und jede Ausgabedatei eingelesen werden.

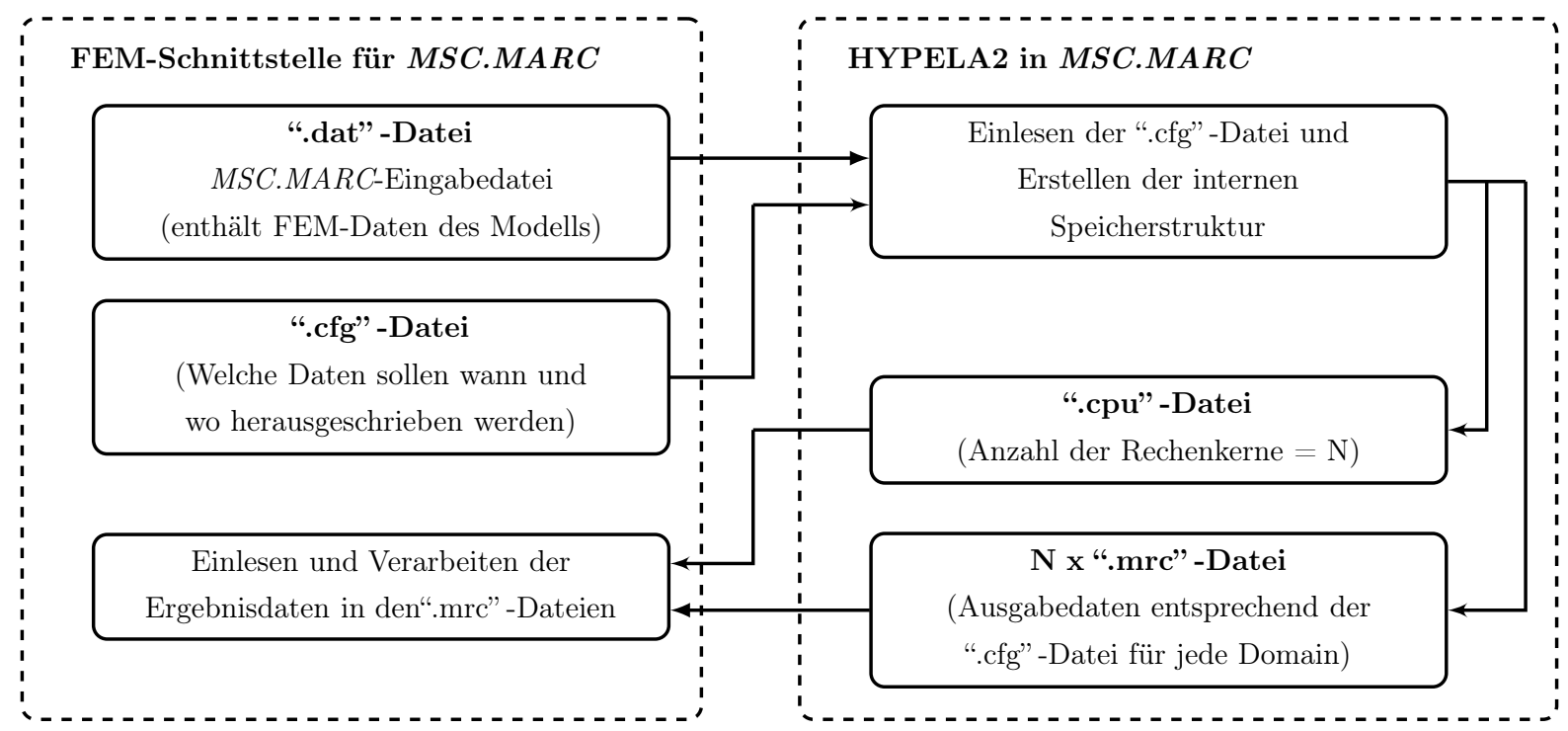

Bild 2.5: Datentransfer bei Nutzung der HYPELA2 in MSC.MARC.

Es besteht jedoch noch die Schwierigkeit, die Parameter in geeigneter Form dem Solver zugänglich zu machen. Dies ist möglich, indem die in MSC.MARC vorhandenen "Initial Conditions "zweckentfremdet und als Transfermedium genutzt werden. Da bei der Definition von Initial Conditions eine Elementzuordnung obligatorisch ist und diese Information auch in die ".dat" -Datei geschrieben wird, können mehrere Materialbereiche definiert und innerhalb der User-Subroutinen unterstützt werden. Dies ist sowohl mit $M A R C$-eigenen Stoffgesetzen, als auch mit nutzereigenen Materialmodellen möglich.

\subsection{Parametrisierung der Modellgeometrie}

Während bei der Stoffgesetzanpassung die Materialparameter im Fokus stehen, gilt bei der Identifikation von Formparametern sowie der Formoptimierung das Interesse der Geometrie eines Bauteils bzw. des zugehörigen Modells. Da in beiden Fällen während der Optimierung eine oder mehrere geometrische Merkmale (meist Randkonturen) gezielt verändert werden sollen, müssen diese zunächst in geeigneter Form parametrisiert werden. Hierfür werden Designvariablen (Formparameter) als unabhängige Veränderliche eingeführt, mit deren Hilfe die zu beschreibenden Konturen eindeutig definiert werden können (vgl. Schumacher (2005); Floriani und Spagnuolo (2008)). Liegt das FEM-Modell bereits in parametrisierter Form (z.B. als PYTHON-Skript bei ABAQUS) vor, können darin enthaltene Parameter wie Radien oder Kantenlängen unmittelbar als Designvariablen fungieren. Dieses Vorgehen ist jedoch ungünstig oder unmöglich, falls

- ein Parameter die zugehörige Kontur nur unzureichend beschreibt,

- durch das Modell bedingte Korrelationen zwischen Parametern auftreten oder 
- das FEM-Modell nicht in parametrisierter Form vorliegt.

Ein übliches Prozedere besteht darin, die zu beschreibenden Konturen der Struktur durch mathematische Funktionen mit den Designvariablen als Steuerparametern auszudrücken. Typisch hierfür ist die Nutzung von Polynomzügen (Splines), welche stückweise aus Polynomen höchstens $n$-ten Grades zusammengesetzt sind. Wichtige Vertreter sind hierbei kubische Splines, Basis-Splines (B-Splines) sowie NURBS (Non-Uniform Rational B-Splines), welche durch den Vektor der Designvariablen $\underline{p}$ charakterisiert werden (Piegl und Tiller, 1995; de Boor, 2001). Im Gegensatz zu B-Splines und NURBS verlaufen kubische Splines durch alle gegebenen Stützstellen. Dadurch lassen sich die Randkonturen vorliegender FEM-Netze mit geringem Aufwand mathematisch ausdrücken. Allerdings ist die direkte Verwendung dieser Stützstellen für die Optimierung nicht sinnvoll, da damit eine Vielzahl von Designvariablen verbunden wäre. Die Konturen auf Basis von B-Splines und NURBS hingegen werden durch Kontrollpunkte (De-Boor-Punkte) gesteuert. Bei Verwendung von NURBS wird jedem Kontrollpunkt zusätzlich ein Wichtungsfaktor zugeordnet. Der hauptsächliche Vorteil bei der Verwendung von NURBS besteht neben der geringeren Anzahl Designvariablen darin, dass sich die Variation einer Designvariablen nur auf einen begrenzten Bereich in der Nähe des Kontrollpunktes auswirkt. Dadurch kann die Korrelation zwischen den Formparametern erheblich verringert werden.

Da in $S P C$-Opt keine grafische Nutzeroberfläche zur Erstellung von FEM-Netzen vorgesehen ist, werden für die Modellerstellung kommerzielle FEM-Programme wie ANSYS oder $M S C$ $M A R C$ genutzt. Mit diesen erstellt der Anwender das Ausgangsnetz und legt die zugehörigen Eigenschaften, beispielsweise die Randbedingungen oder verwendeten Materialmodelle, fest. Für die Optimierung werden die relevanten Konturen während der Modellerstellung durch Geraden, kubische Splines oder eine hinreichende Anzahl Keypoints approximiert. Die den Konturen zugeordneten Knoten werden in Knoten-Sets zusammengefasst und eindeutig benannt. Anschließend wird das Modell im dem FEM-Programm entsprechenden Datenformat gespeichert und mittels des in Kap. 2.2 vorgestellten Konvertierungsprogramms in das für $S P C$-Opt lesbare ".sim"-Format umgewandelt. Der Konverter enthält unter anderem einen Algorithmus von Schneider (1993), mit welchem ohne merklichen Genauigkeitsverlust eine Darstellung der Geraden, kubischen Splines oder Keypoint-Gruppen durch NURBS möglich ist. Während dieses Prozesses werden die Koordinaten und Wichtungen der De-Boor-Punkte berechnet, aus denen der Anwender diejenigen Größen auswählt, welche als Designvariablen fungieren sollen.

In Bild 2.6 ist der Ausschnitt eines FEM-Netzes dargestellt. Die Randkontur des inneren Kreises wird durch kubische Splines approximiert, deren Stützstellen den Eck- und Mittelknoten der Elementkanten entsprechen. In den gemeinsamen Knoten zweier Elementkanten gilt Stetigkeit. Bei Konturen, die nicht geschlossen sind, wird im Anfangs- und Endpunkt der Anstieg vorgegeben. Der nach der Konvertierung vorliegende NURBS wird durch die eingezeichneten De-Boor-Punkte charakterisiert.

Falls die Optimierung für räumliche Probleme durchgeführt werden soll, kann die beschriebene Vorgehensweise ebenso angewendet werden. Dies erfolgt, indem die Konturen durch NURBS-Flächen approximiert und die zugehörigen De-Boor-Punkte in analoger Form für die Optimierung verwendet werden (Elsässer, 1998; Friedrich, 2011). 


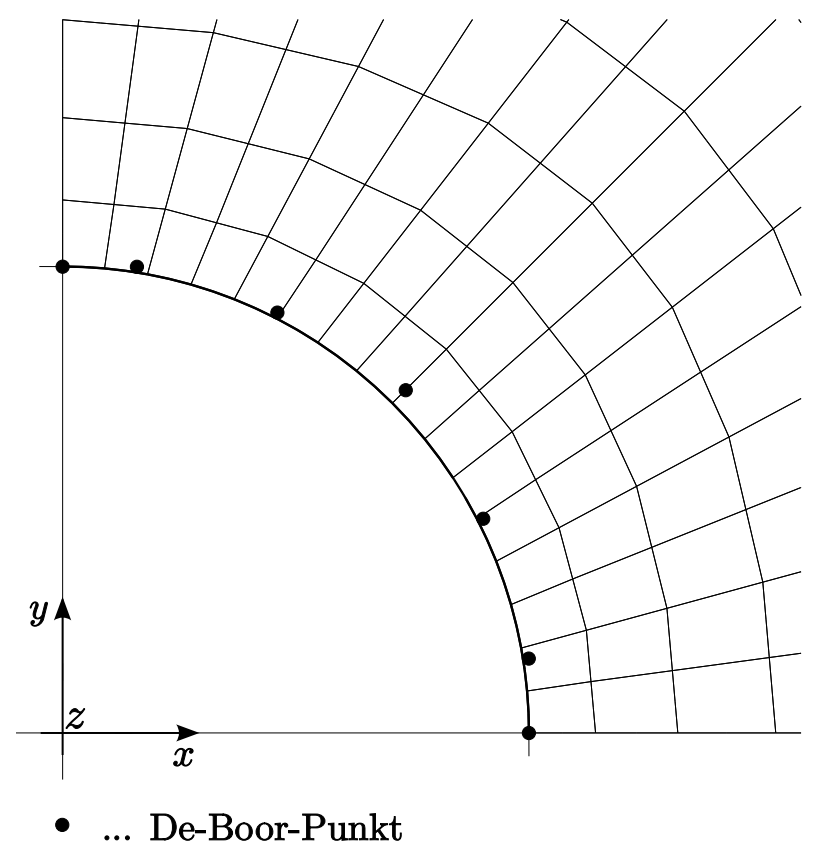

Bild 2.6: Darstellung einer Randkontur mittels kubischer Splines und NURBS.

Als Voraussetzung für die Durchführung von FEM-Simulationen müssen in jedem Optimierungsschritt FEM-Netze auf Basis derjenigen Konturen generiert werden, welche sich auf den jeweils aktuellen Parametersatz bzw. die gestörten Parametersätze beziehen. Grundsätzlich kann dies auf verschiedene Arten erfolgen.

Eine Möglichkeit besteht darin, das benötigte FEM-Netz für jede gestörte Kontur automatisch neu zu vernetzen. Werden kommerzielle CAD-Programme wie ${ }^{N X}{ }^{*}$ und $\mathrm{PTC}^{\mathrm{C} C \mathrm{Co}^{*}}$ verwendet, stehen solche automatischen Vernetzungswerkzeuge bereits zur Verfügung und können in den Optimierungsalgorithmus integriert werden. Zudem entspricht in den meisten Fällen die mathematische Beschreibung der Konturen auch den entsprechenden CAD-Daten. Somit sind die Formparameter des CAD-Modells direkt mit den Designvariablen gekoppelt. Dadurch liegt nach der Optimierung die Kontur des Modells direkt in Form der CAD-Daten vor und eine Konvertierung erübrigt sich. Der Nachteil dieser Vorgehensweise ist jedoch, dass in jedem einzelnen Optimierungsschritt für jede Parameterstörung ein neues FEM-Netz automatisch erstellt wird und dabei unterschiedliche Netztopolgien (Veränderung der Anzahl der Knoten) auftreten können.

Da zu Beginn der Optimierung oft ein der Startkontur entsprechendes FEM-Netz als Ausgangsbasis vorliegt, ist ein anderer Weg möglich. Dabei bleibt die Netztopologie unverändert und es erfolgt lediglich eine Variation der Knotenkoordinaten während der Optimierung. Die Qualität der neu gewonnenen FEM-Netze hängt dabei stark vom mathematischen Algorithmus ab, mit welchem die Neuberechnung der Knotenkoordinaten durchgeführt wird. Eine Variante stellt die geeignete Verschiebung der Knoten des Ausgangsnetzes dar (Harzheim, 2008). Ausgehend von dem Vektor der Anfangskoordinaten $\underline{R}^{0}$ lässt sich der neue Vektor der Knotenkoordinaten $\underline{R}$ durch den Formbasisvektor $\underline{T}$ und die Designvariable $p$ darstellen:

$$
\underline{R}=\underline{R}^{0}+p \underline{T}
$$

*Detaillierte Angaben sind im Anhang [A.1] aufgeführt. 
Für mehrere Designvariablen gilt

$$
\underline{R}=\underline{R}^{0}+\sum_{i=1}^{N} p_{i} \underline{T}_{i} .
$$

Alle Designvariablen befinden sich in dem Raum, welcher durch die Formbasisvektoren $\underline{T}$ aufgespannt wird. Im Verlauf der Optimierung werden deren Werte so bestimmt, dass der Wert der Zielfunktion minimal wird. Die Ermittlung der Basisvektoren muss derart erfolgen, dass Netzverzerrungen infolge von Änderungen der Designvariablen nicht zu Diskretisierungsunterschieden führen, aufgrund derer die Ergebnisse der FEM-Simulation verfälscht werden. Da die Berechnung der Formbasisvektoren meist mit einem hohen Aufwand (z.B. Simulation typischer Lastfälle zur besseren Abschätzung) verbunden ist, existieren nur wenige kommerzielle Programme wie $M S C . N A S T R A N^{*}$ zur Generierung.

Ein ähnlicher, flexiblerer Ansatz besteht darin, die Koordinaten der FEM-Knoten an den in der Nähe befindlichen Konturen auszurichten. Vergleichbar ist dies mit der Funktionsweise von grafischen Programmen zur Verzerrung von Grafiken. Im Gegensatz zur Methode der Basisvektoren existieren hier mehrere leistungsfähige kommerzielle Programme, z.B. die Morphing-Tools von $A N S A{ }^{*}$ und HYPERMESH* ${ }^{*}$. Auch die Vorgehensweise in SPC-Opt basiert auf dieser Grundidee.

Während der Optimierung ändern sich zunächst die Koordinaten (und Wichtungen) der DeBoor-Punkte und damit die zugehörigen Konturen. In Folge dessen müssen auch die Randund Innenknoten des FEM-Netzes dementsprechend verschoben werden. In den folgenden Ausführungen, die sich auf das FEM-Netz beziehen, wird nur noch von "Knoten" gesprochen. Diesen werden im Rahmen der Netzgenerierung lokale Koordinatensysteme (KS) zugewiesen. Typ sowie Koordinatenursprung können frei gewählt werden. Wenn kein lokales KS definiert wird, dient das globale kartesische als Bezugssystem. Wenn eine oder mehrere Koordinatenachsen eines Punktes $P$ bei der Aktualisierung der Netztopologie berücksichtigt werden sollen, müssen jeweils zwei Bezugskurven für jede Koordinatenachse definiert werden, sodass sich $P$ zwischen diesen befindet.

In Bild 2.7 a ist die Kombination von Geraden $\left(B_{\eta 1}, B_{\eta 2}\right)$ und kubischen Splines $\left(B_{\xi 1}, B_{\xi 2}\right)$ als Bezugskurven in einem ebenen Modell dargestellt. Die Abstände von $P$ zu den Bezugskurven $d_{\xi 1}, d_{\xi 2}$ sowie $d_{\eta 1}$ und $d_{\eta 2}$ führen auf die Verhältnisse

$$
\gamma_{1}=\frac{d_{\xi 1}}{d_{\xi 1}+d_{\xi 2}} \quad \text { und } \quad \gamma_{2}=\frac{d_{\eta 1}}{d_{\eta 1}+d_{\eta 2}}
$$

welche für jeden Knoten gespeichert werden. Sie bilden die Basis zur Bestimmung der neuen Koordinaten bei Änderung der Bezugskurven. Im Rahmen des entwickelten Verfahrens stellt die Einhaltung der Streckenverhältnisse (G1. 2.3) eine notwendige Bedingung bei der Netzaktualisierung dar. Um den Anwendungsbereich der Methode zu erweitern, können auch lokale Polarkoordinatensysteme einbezogen werden (Bild 2.7b).

${ }^{*}$ Detaillierte Angaben sind im Anhang [A.1] aufgeführt. 


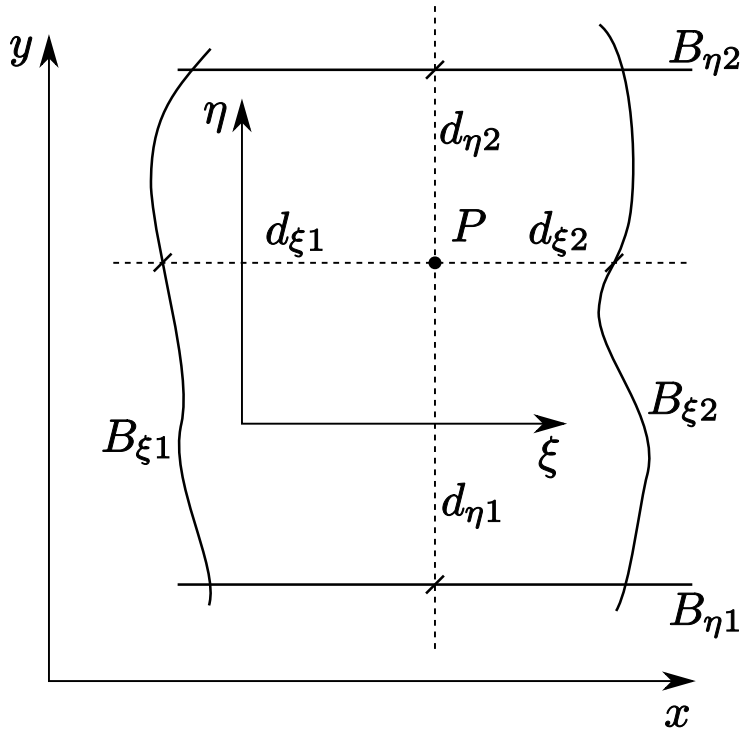

(a) in lokalen kartesischen Koordinaten

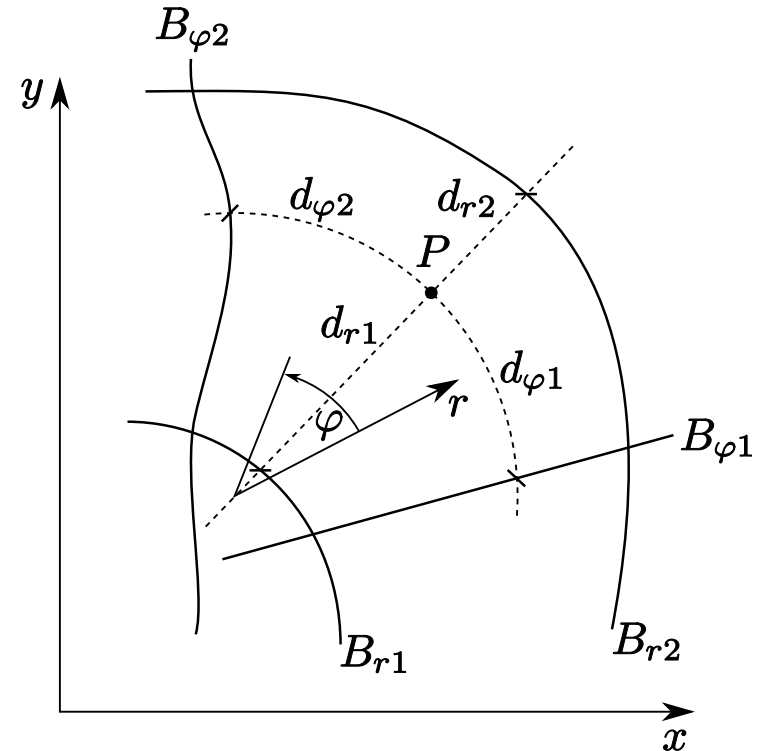

(b) in lokalen Polarkoordinaten

Bild 2.7: Abstände eines Knotens $P$ von den Bezugskurven.

Die Anwendung des Verfahrens auf räumliche Probleme ist mit SPC-Opt in gleicher Weise möglich. Der Unterschied besteht lediglich darin, dass der Anwender den Knoten keine Bezugskurven, sondern Bezugsflächen zuordnet. Es muss jedoch sichergestellt werden, dass die Koordinatenachsen einen Schnittpunkt mit den zugeordneten Bezugsflächen besitzen.

In Bild 2.8 ist ein FEM-Netz im Ausgangszustand und nach der Netzaktualisierung bei Änderung von drei Bezugskurven angegeben. Im dargestellten Beispiel entsprechen die lokalen Koordinatensysteme der Knoten dem globalen kartesischen KS. Die Topologie des Netzes bleibt erhalten.
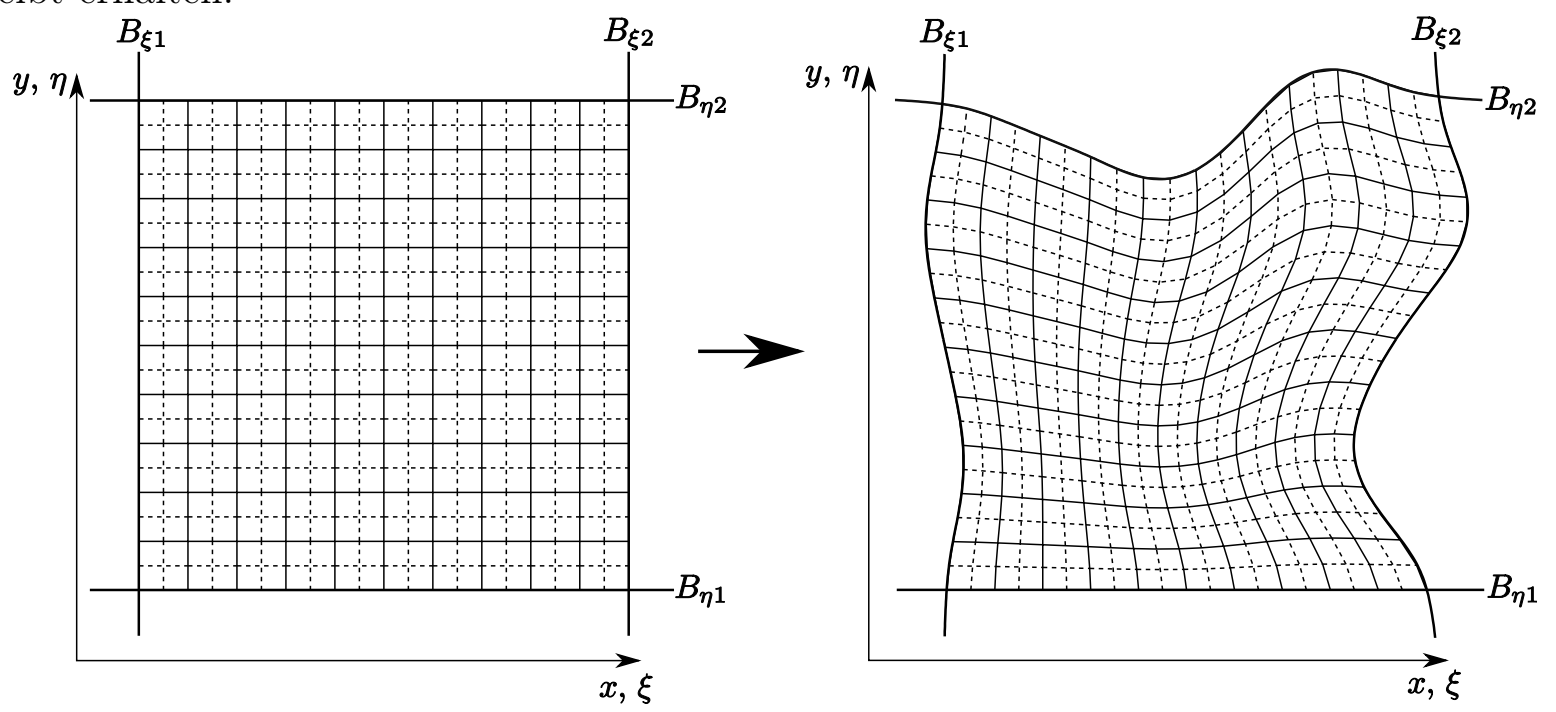

Bild 2.8: Netzaktualisierung eines FEM-Netzes nach Veränderung von drei Bezugskurven.

Für komplexe Geometrien ist es oft sinnvoll, das Netz in unterschiedliche Bereiche aufzuteilen und diesen jeweils andere Bezugskurven bzw. -flächen zuzuweisen. Dabei können benachbarte Gebiete auch durch eine Bezugskontur getrennt werden, welche beiden Bereichen (evtl. mit unterschiedlichen lokalen KS) zugeordnet ist. 
Bei der Implementierung der Methode stand im Vordergrund, dass auch das verzerrte Netz eine hohe Qualität besitzt, jedoch gleichzeitig der benötigte Rechenaufwand möglichst klein ist. Es wurde deshalb bewusst kein Ansatz verwendet, welcher auf der Beibehaltung eines Gleichgewichtszustandes zwischen den Knoten basiert. Der hier dargestellte Algorithmus ermöglicht es, für jeden Parametersatz mit geringem Aufwand ein valides FEM-Netz analytisch zu erzeugen. Ein Nachteil des Verfahrens besteht allerdings darin, dass der Anwender zwar nur selten neue Bezugskonturen definieren muss (oft bietet sich die Nutzung von bestehenden Randkonturen an), der Arbeitsaufwand zur sinnvollen Gruppierung von Knoten und die Erzeugung von lokalen KS (falls sinnvoll) jedoch unumgänglich ist.

In der Regel werden durch das Verfahren qualitativ gute, wenn auch nicht ideale, FEM-Netze (im Sinne der geometrischen Elementgestalt) erstellt. Große Änderungen der Randkonturen, bereits vorhandene spitze Innenwinkel oder große Seitenverhältnisse können jedoch dazu führen, dass bei der Simulation Diskretisierungsfehler entstehen oder verstärkt werden. Ergänzend zum bestehenden Algorithmus stehen dem Anwender deshalb iterative Verfahren zur nachträglichen Glättung der FEM-Netze zur Verfügung. Diese bestehen aus einer Kombination geeigneter Glättungsalgorithmen mit entsprechenden Bewertungskriterien.

Bei der Implementierung wurden Laplace- und Optimierungsbasiertes Smoothing berücksichtigt (Blacker und Stephenson, 1991; Canann et al., 1993, 1998; Thompson et al., 1998; Mukherjee, 2002; Chen et al., 2003). Darüber hinaus besteht die Möglichkeit zur Kombination beider Verfahren, wodurch die Vorteile von kurzer Rechenzeit mit der erhöhten Flexibilität bei der Suche idealer Knotenpositionen verknüpft werden können. Alle verfügbaren Algorithmen eignen sich sowohl für strukturierte als auch unstrukturierte Gitter und sind unabhängig vom Elementtyp.

Im Kontext der Elementbewertungsfunktionen, die stets elementbezogene Gültigkeit aufweisen, sind eine Reihe verschiedener Metriken für Dreiecks-, Vierecks- und Hexaederelemente im Programm integriert (Canann et al., 1993; Knupp, 2002; Branets und Carey, 2005). Durch die Kombination von Qualitätsmetriken mit den Glättungsalgorithmen und Variation der Steuerparameter kann das Ergebnis der Netzglättung gezielt beeinflusst werden.

Es hat sich gezeigt, dass starke Netzglättungen einen Einfluss auf das Ergebnis der FEMSimulation haben, wenn auch in deutlich geringerem Maße als die Diskretisierungsunterschiede bei Neuvernetzungsstrategien. Da die Netzglättung gewöhnlich auf alle durch Parametervariation entstandenen FEM-Netze gleichermaßen angewendet wird, bleibt der relative Unterschied zwischen diesen FEM-Netzen davon unberührt. Der entscheidende Vorteil gegenüber Neuvernetzungsstrategien besteht deshalb darin, dass die bei der Formoptimierung mit Gradientenverfahren notwendige stetige Änderung der Knotenkoordinaten bei der Variation von Bezugskurven gewährleistet ist.

\subsection{Parallelisierung}

Der zeitliche Aufwand für die Lösung der benötigten FEM-Simulationen hängt maßgeblich von der verfügbaren Rechenleistung ab. Eine Vergrößerung der Rechenkapazität wird durch die Verwendung von mehreren Rechenknoten ("Processing Elements", PE) in einer parallelen Rechnerarchitektur erreicht. In den vergangenen Jahren hat die Verbreitung dieser Technik 
deutlich zugenommen. Selbst handelsübliche Heimcomputer besitzen vier oder mehr Rechenkerne. Um die Rechenkapazität sinnvoll nutzen zu können, werden die zu lösenden Aufgaben in unabhängige Teilprobleme aufgeteilt und parallel bearbeitet.

Derzeit stellen lokale Netzwerke unabhängiger Computer ("Networks of independent Computers", NoCs) die häufigste Art paralleler Architekturen dar. Nahezu alle Firmen und wissenschaftliche Einrichtungen verfügen über eine Vielzahl von Computern, welche durch ein lokales Netzwerk miteinander verbunden sind. Gewöhnlich bestehen diese aus heterogenen Gruppen von Computern (Hennessy und Patterson, 2003; Parhami, 2005; Silc et al., 1999). Der hohe Verbreitungsgrad solcher Systeme stellt einen großen Vorteil gegenüber den spezialisierten massiv-parallelen Computern ("massively parallel processor computers", MPP) dar. Dem entgegen steht der meist größere Programmieraufwand paralleler Umgebungen für NoCs. Aufgrund der gewachsenen Strukturen von NoCs sind diese gewöhnlich aus Systemen mit unterschiedlichen Prozessortypen und Geschwindigkeiten aufgebaut. Darüber hinaus kann die Anzahl der verfügbaren PEs variieren. Idealerweise muss ein Steuerprogramm fähig sein, die Berechnungen und Kommunikation ungleichmäßig unter den PEs mit Berücksichtigung der jeweiligen Rechenleistung zu verteilen und mit Ausfällen im Netzwerk umzugehen (Lastovetsky, 2003). Eine weitere nachteilige Eigenschaft ist der physisch verteilte Speicher. Dadurch verringert sich zwar die Zugriffszeit auf lokaler Ebene, der Austausch von Daten zwischen den PEs ist jedoch aufwändig und mit deutlichen Latenzen verbunden.

Im Hinblick auf SPC-Opt ist die Nutzung paralleler Rechentechniken auf zwei Ebenen sinnvoll. So können die FEM-Berechnungen üblicher Weise durch programmeigene Möglichkeiten parallelisiert werden, wobei dies meist durch die Anzahl der zur Verfügung stehenden Lizenzen begrenzt ist. Darüber hinaus ist es möglich, mehrere Instanzen von SPC-Opt zu starten, wobei eine Instanz die Funktion der Verwaltung (Master) übernimmt. Entsprechend Bild 2.9 können verschiedene Experimente in der Optimierung berücksichtigt werden, wobei für jedes dieser Experimente entsprechend der Parameteranzahl mehrere FEM-Berechnungen (in Bild 2.9 als "Direct" bezeichnet) durchgeführt werden müssen. Diese werden in einer Warteschlange (Queue) gesammelt und durch die übrigen Prozesse (Slaves) abgearbeitet.

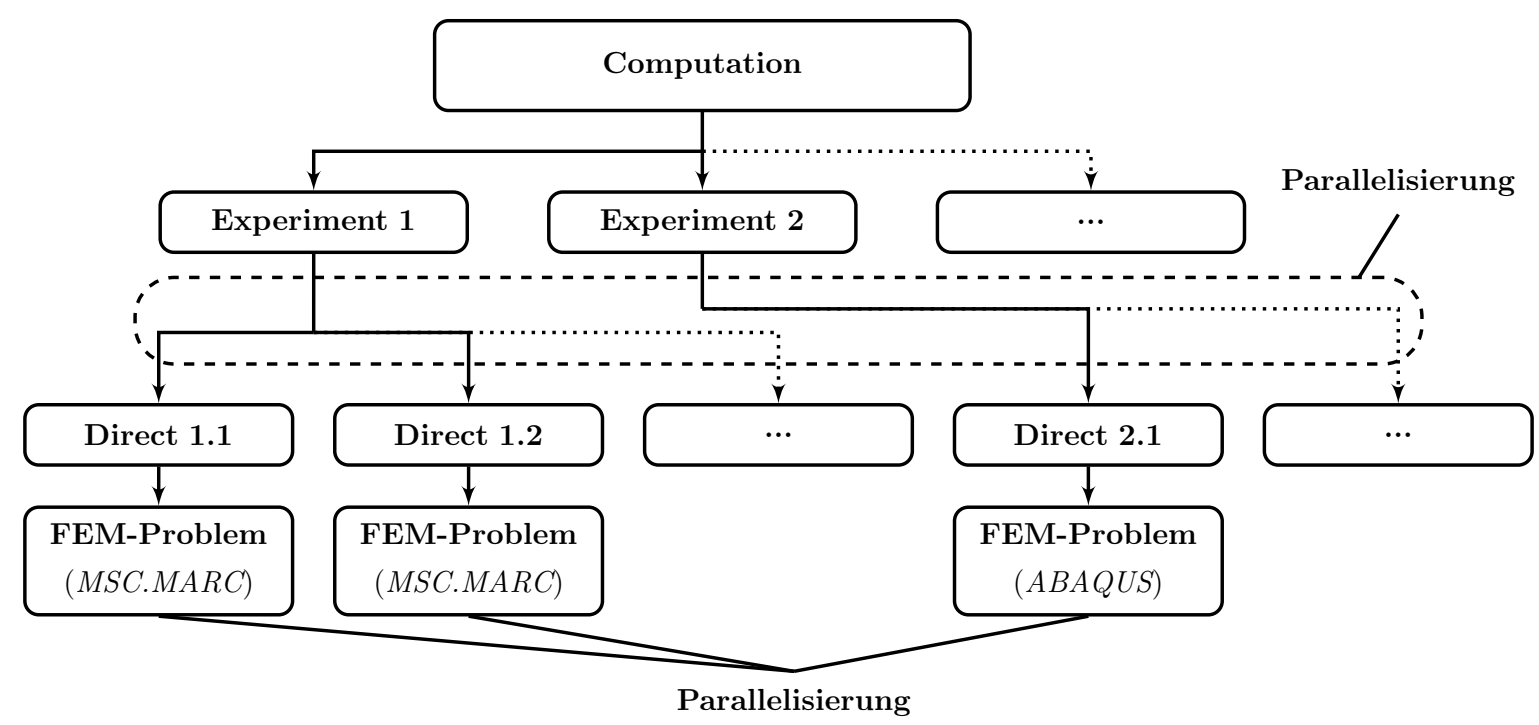

Bild 2.9: Darstellung der Möglichkeiten zur Parallelisierung. 
An der TU-Chemnitz steht der hochparallele Rechner ("High Performance Cluster", HPC) CHiC ("Chemnitzer High Performance Cluster") als Testplattform zur Verfügung. Die Kommunikation erfolgt durch ein gemeinsames Infiniband (10 Gbit/s) mit geringer Latenz und großer Bandbreite. Im Gegensatz zu NoCs haben alle Prozessoren die gleiche Geschwindigkeit und garantieren einen hohen Grad an Verfügbarkeit. Darüber hinaus führen die geringe Latenz und große Bandbreite des Netzwerks, verglichen mit heterogenen NoCs, zu einer größeren Geschwindigkeit bei der Kommunikation und dem Datenaustausch zwischen den PEs des HPC.

Die Rechenknoten kommunizieren über das Message Passing Interface (MPI), eine Standardbibliothek für den Nachrichtenaustausch in parallelen Systemen (Lastovetsky, 2003; Snir, 1998). Das MPI gewährleistet eine hohe Effizienz sowohl in MPPs als auch NoCs und stellt die notwendigen Sende- und Empfangs-Routinen für die Point-to-Point Kommunikation bereit.

Um Fehler während der Nachrichtenübermittlung zu vermeiden, müssen dem Empfänger Typ und Größe des zu sendenden Datenpaketes bekannt sein. Des Weiteren ist es erforderlich, dass der Speicher des Datenpaketes zusammenhängend vorliegt. Speziell bei der Verwendung großer Datenfelder variabler Größe kann das schwierig sein, weshalb die $\mathrm{C}++$ Bibliothek BLITZ* genutzt wird. Deren Templates bieten eine hohe Effizienz bei der dynamischen Speicherverwaltung und unterstützen gleichzeitig verschiedene Matrizenoperationen. Trotz dessen kann es insbesondere bei der Verwendung einer großen Zahl PEs zu Fehlern bei der Datenübertragung oder anfallenden Dateioperationen kommen. Zusätzlich können während einer Optimierung ungünstige Parameterkonstellationen auftreten, welche die erfolgreiche Konvergenz der Simulation verhindern. In SPC-Opt gibt es deshalb ein dynamisches Reaktionsschema, welches in Abhängigkeit von der Art des Abbruchs einer Simulation entscheidet, ob die Optimierung abgebrochen, ein eventuell defekter Rechenknoten deaktiviert oder eine neue Instanz der Simulation gestartet wird.

${ }^{*}$ Detaillierte Angaben sind im Anhang [A.1] aufgeführt. 


\section{Parameteridentifikation}

\subsection{Optimierungsaufgabe}

Ein Großteil der aktuell verfügbaren FEM-Programme stellt zur Darstellung des Materialverhaltens eine Vielzahl verschiedener Stoffgesetze zur Verfügung. Für diese müssen jedoch Materialparameter ausgewählt werden, mit denen das Verhalten der real verwendeten Materialien möglichst gut abgebildet werden kann. Andernfalls sind die Ergebnisse der zugehörigen FEM-Simulation nicht vertrauenswürdig.

Um diese Parameter zu bestimmen, wird in den meisten Fällen eine Materialparameteridentifikation, welche ein inverses Problem darstellt (Mahnken und Stein, 1996; Moritz, 1993; Schnur und Zabaras, 1992), durchgeführt. Eine analytische Lösung des Problems ist in der Regel nicht möglich, da sich Materialparameter nicht direkt messen sondern lediglich anhand ihrer Wirkung im Experiment analysieren lassen. Eine Lösung stellt die Rückführung des Problems auf eine Optimierungsaufgabe dar. Dabei ist insbesondere die Methode der Fehlerquadratminimierung (FQM) geeignet, um die Parameter $p_{j}(j=1, \ldots, n)$ von konstitutiven Gleichungen zu bestimmen. Dafür werden zu bestimmten Beobachtungszeitpunkten $t_{i}$ an festgelegten Orten $\underline{R}_{i}^{B}$ die Residuen zwischen den fehlerbehafteten Messwerten $\bar{y}\left(t_{i}\right)=\bar{y}_{i}$ am Bauteil und dem zugehörigen Modell $M\left(p_{j}, \underline{R}_{i}^{B}, t_{i}\right)=y_{i}\left(p_{j}\right)$ gebildet (Nocedal und Wright, 1999):

$$
\begin{aligned}
\bar{r}_{i}\left(p_{j}\right)=\bar{y}_{i}-M\left(p_{j}, \underline{R}_{i}^{B}, t_{i}\right) & =\bar{y}_{i}-y_{i}\left(p_{j}\right) \\
\operatorname{mit} i & =1, \ldots, m \\
\text { und } j & =1, \ldots, n .
\end{aligned}
$$

Hierbei wird das Modell $M$ oft durch Größen repräsentiert, welche im Rahmen einer numerischen Simulation berechnet wurden. Die Nutzung analytischer Modelle in Form von mathematischen Berechnungsvorschriften ist jedoch ebenso möglich. Gesucht wird nunmehr derjenige Parametersatz $p_{j}^{*}$, für welchen eine auf den Residuen $\bar{r}_{i}$ basierende Zielfunktion $\Phi\left(p_{j}\right)$ minimal wird:

$$
\text { Suche } p_{j}^{*} \text {, sodass } \Phi\left(\bar{r}_{i}\left(p_{j}\right)\right)=\Phi\left(p_{j}\right) \rightarrow \min _{p}
$$

Gewöhnlich wird zur Berechnung der Zielfunktion die Fehlerquadratsumme der Residuen eines Versuches gebildet. Dabei ist zu beachten, dass die Residuen unterschiedliche Einheiten und Größenordnungen besitzen können. Die einheitenbehafteten $\bar{r}_{i}$ werden daher durch die Wichtungsfaktoren $w_{i}$ auf einheitenfreie und bei Bedarf skalierte Residuen $r_{i}$ reduziert:

$$
r_{i}=w_{\underline{i}} \bar{r}_{i}
$$


Mit diesen lässt sich die Fehlerquadratsumme und somit die Zielfunktion bilden:

$$
\Phi\left(p_{j}\right)=\frac{1}{2} \sum_{i=1}^{m}\left[r_{i}\left(p_{1}, \ldots, p_{n}\right)\right]^{2} \rightarrow \min _{p} .
$$

Unter der Annahme, dass die Zielfunktion hinreichend glatt ist, bietet sich zur Lösung des Optimierungsproblems ein gradientenbasiertes Verfahren an. Hierfür wird zunächst $p$ durch die dimensionslose Parametermatrix $x$ ersetzt und Gl. 3.4 in typisch mathematischer Form formuliert (Nocedal und Wright, 1999):

$$
\text { Suche } x \in \mathbb{R}^{n}, \text { sodass } f(x)=\frac{1}{2} \sum_{i=1}^{m}\left[r_{i}(x)\right]^{2} \rightarrow \min _{x}
$$

wobei $f(x)$ die Zielfunktion, $x$ die Parametermatrix und $r_{i}$ die Einträge der Residuenmatrix $r$ darstellen. Zwecks Verwendung gradientenbasierter Verfahren muss durch Ableitung der Residuen $r_{i}$ nach den Parametern $x_{j}$ die Jacobimatrix $J$ bestimmt werden:

$$
J:=J_{i j}=\frac{\partial r_{i}}{\partial x_{j}}
$$

Zusammen mit Gl. 3.5 folgt unter Nutzung der Produktregel:

$$
\begin{aligned}
\nabla f(x) & =J^{T} r \text { und } \\
\nabla^{2} f(x) & =J^{T} J+\sum_{i=1}^{m} r_{i} \nabla^{2} r_{i},
\end{aligned}
$$

wobei der Nabla-Operator aufgrund der Ableitung nach den entdimensionierten $x$ einheitenfrei ist. Ausgehend von einem Startpunkt $x_{0}$ wird versucht, mittels Iteration ein $x^{*} \mathrm{zu}$ ermitteln, für welches die Funktion $f\left(x^{*}\right)$ ein Minimum darstellt. Dabei wird $x$ in folgender Weise aufdatiert:

$$
x_{k+1}=x_{k}+d_{k} \text { mit } k=0,1,2, \ldots .
$$

Es gilt nun, in jedem Schritt $k$ den Korrekturvektor $d_{k}$ derart zu bestimmen, dass möglichst wenige Iterationen erforderlich sind. Dabei wird gefordert, dass nur diejenigen $d_{k}$ akzeptiert werden, durch welche $f\left(x_{k+1}\right)$ kleiner als $f\left(x_{k}\right)$ wird, also ein Abstieg sichergestellt ist. Entsprechend dieser Forderung und bei Wahl der passenden Schrittlänge wird

$$
d_{k}^{T} \nabla f\left(x_{k}\right)<0
$$

erfüllt. Des Weiteren gilt für $f(x)$ im Minimum $x^{*}$ die Optimalitätsbedingung 1. Ordnung:

$$
\nabla f\left(x^{*}\right)=0
$$

Demnach muss für Gl. 3.10 das Nullstellenproblem gelöst werden, was beispielsweise durch die Anwendung des Newton-Iterationsverfahrens erfolgen kann. Hierbei wird eine Nullstelle von einer Funktion $F(x)$ ermittelt. Die typische Newton-Iteration für die Gleichung $F(x)=0$ sieht bekanntermaßen wie folgt aus:

$$
x_{k+1}=x_{k}-F^{\prime}\left(x_{k}\right)^{-1} F\left(x_{k}\right),
$$


wobei $F(x)$ gerade $\nabla f(x)$ darstellt und $F^{\prime}$ die Ableitungsmatrix von $F$ nach $x_{k}$ ist. Aufgrund der Gestalt von Gl. 3.8 ergibt sich automatisch der Newton-Korrekturschritt

$$
d_{k}^{N}=-\left(\nabla^{2} f\left(x_{k}\right)\right)^{-1} \nabla f\left(x_{k}\right)
$$

Einen weiteren Zugang stellt die Entwicklung eines quadratischen Ersatzmodells für die Zielfunktion im Punkt $x_{k}$ in Form einer Taylor-Reihe bis zum quadratischen Glied dar:

$$
f\left(x_{k+1}\right) \approx m_{k}(x)=f\left(x_{k}\right)+\left(x-x_{k}\right)^{T} \nabla f\left(x_{k}\right)+\frac{1}{2}\left(x-x_{k}\right)^{T} \nabla^{2} f\left(x_{k}\right)\left(x-x_{k}\right) .
$$

Anstatt des Minimums der Zielfunktion $f(x)$ wird nunmehr das Minimum von $m_{k}(x)$ gesucht. Dies erfordert, dass in analoger Vorgehensweise die Ableitung $\nabla m_{k}(x)$ Null wird:

$$
\nabla m_{k}(x)=\nabla f\left(x_{k}\right)+\nabla^{2} f\left(x_{k}\right)\left(x-x_{k}\right)=0 .
$$

Die Anwendung von Gl. 3.8 auf Gl. 3.14 führt wieder zum Newton-Korrekturschritt $d_{k}^{N}$ nach Gl. 3.12 ,

Unabhängig vom gewählten Zugang ist die Ermittlung von $\nabla^{2} f(x)$ notwendig, was jedoch sehr aufwändig ist. Es wird deshalb eine approximierte Hesse-Matrix $B_{k}$ eingeführt. Das Einsetzen in Gl. 3.13 liefert

$$
m_{k}(x)=f\left(x_{k}\right)+\left(x-x_{k}\right)^{T} \nabla f\left(x_{k}\right)+\frac{1}{2}\left(x-x_{k}\right)^{T} B_{k}\left(x-x_{k}\right)
$$

und der entsprechende Newton-Korrekturschritt aus Gl. 3.12 lautet:

$$
d_{k}^{N}=-B_{k}^{-1} \nabla f\left(x_{k}\right) .
$$

Die Berechnung von $B_{k}$ kann grundsätzlich auf vier verschiedene Weisen erfolgen, welche in Kap. 3.3-3.6 diskutiert werden.

\subsection{Gewichtete Fehlerquadratkennzahl (GFK)}

Im Allgemeinen stellt der Zielfunktionswert, welcher im Rahmen der Identifikation minimiert wird, eine (oft gewichtete) Kombination aus Zielfunktionswerten unterschiedlicher Versuche dar. Diese Versuche unterscheiden sich jedoch häufig voneinander bezüglich des Belastungstyps, der Anzahl der Lastschritte und der Belastungsintensität. Um dennoch eine resultierende Zielfunktion aufstellen zu können, müssen die einzelnen Zielfunktionsanteile entsprechend der jeweiligen Belastungsart vor dem Zusammenführen gezielt modifiziert werden. Es wird deshalb ein Qualitätskriterium benötigt, mit welchem sowohl verschiedene Versuche als auch unterschiedliche Identifikationsergebnisse miteinander verglichen werden können. Das Kriterium sollte folgende Bedingungen erfüllen:

- Unabhängigkeit vom Belastungstyp

- Unabhängigkeit von der Anzahl der Lastschritte

- Unabhängigkeit von der Belastungsintensität 
Ein Kriterium, welches diese Bedingungen erfüllt, ist die sogenannte "Gewichtete Fehlerquadrat-Kennzahl" (GFK), welche auf Basis der Anzahl der in die Identifikation einfließenden Lastschritte $n_{L}$, der gemessenen Größen $\bar{y}_{i}$ sowie den zugeordneten Simulationsgrößen $y_{i}$ wie folgt berechnet wird:

$$
\mathrm{GFK}=\frac{\frac{1}{n_{L}} \cdot \sum_{i=1}^{n_{L}}\left(\bar{y}_{i}-y_{i}\right)^{2}}{[\max (\bar{y})-\min (\bar{y})]^{2}} .
$$

Bei Betrachtung der Bildungsvorschrift wird deutlich, dass diese analog zu Gl. 3.4 die Fehlerquadratsumme der Residuen beinhaltet, wobei jeweils gleichartige $\bar{y}_{i}$ mit gleichen Einheiten in einer GFK berücksichtigt werden. Je kleiner demnach die GFK ist, desto besser stimmen Simulation und Messung überein. Eine Überprüfung zeigt, dass die GFK eine dimensionslose Größe ist, welche nicht mehr unmittelbar durch Belastungstyp, Anzahl der Lastschritte oder die Belastungsintensität "verfälscht" wird. Dadurch können die GFKs verschiedener Versuche quantitativ miteinander verglichen oder zusammengeführt werden. Die quadratische Bestrafung der Abweichung von den Messdaten soll bei der Zusammenführung jedoch ausgeklammert werden, da sonst diejenigen Experimente, deren Informationen zum Materialverhalten am wenigsten den übrigen Versuchen entsprechen würden, einen noch höheren Anteil am Zielfunktionswert hätten. Somit ergibt sich ein übergeordnetes Gütemaß in Form der kombinierten gewichteten Fehlerquadrat-Kennzahl $\overline{\text { GFK}}$, welches auch in der Praxis als Zielfunktionswert genutzt werden kann:

$$
\overline{\mathrm{GFK}}=\left(\frac{1}{n_{e x p}} \cdot \sum_{i=1}^{n_{\exp }} \sqrt{\mathrm{GFK}_{i}}\right)^{2} .
$$

Die abschließende Quadrierung des Terms in Gl. 3.18 ermöglicht es, die berechnete $\overline{\text { GFK }}$ wieder direkt mit den GFK der einzelnen Versuche zu vergleichen.

\subsection{Gradientenverfahren (Verfahren des steilsten Abstiegs)}

Beim Gradientenverfahren entspricht die approximierte Hesse-Matrix der Einheitsmatrix I und führt auf:

$$
B_{k}=I \text {. }
$$

Das heißt, als Schrittrichtung wird immer die Richtung des steilsten Abstieges an der aktuellen Stelle $x_{k}$ gewählt. Der große Vorteil des Verfahrens besteht darin, dass es sicher zum Ziel konvergiert. Dies geschieht allerdings nur langsam. Des Weiteren können ungünstige Zick-Zack-Effekte auftreten, indem die Iterationspunkte hin- und herspringen, obwohl ein einfacher Schritt in eine andere Richtung schon genügen würde.

\subsection{Newton-Verfahren}

Bei Verwendung des Newton-Verfahrens stellt $B_{k}$ den bereits bekannten Term $\nabla^{2} f\left(x_{k}\right)$ dar:

$$
B_{k}=\nabla^{2} f\left(x_{k}\right) \text {. }
$$


Die Nutzung des Newton-Korrekturschrittes kann es ermöglichen, unter Ausnutzung der lokalen quadratischen Konvergenz bei einem Start nahe dem Ziel selbiges sehr schnell zu erreichen. Der Start an einem weit entfernten Punkt verlangsamt das Verfahren jedoch beträchtlich oder lässt es sogar divergieren. Zudem ist, wie bereits erläutert, die Berechnung von $\nabla^{2} f\left(x_{k}\right)$ sehr aufwändig.

\subsection{Gauss-Newton-Verfahren}

Das Gauss-Newton-Verfahren basiert auf einer Vereinfachung des zweiten Terms von Gl. 3.7.

$$
B_{k}=J_{k}^{T} J_{k}
$$

Durch die Vernachlässigung des Terms $\sum_{i=1}^{m} \nabla^{2} r_{i}(x) r_{i}(x)$ ist der Berechnungsaufwand von $B_{k}$ äußerst gering, da lediglich die ersten Ableitungen zur Bestimmung von $J_{k}$ ermittelt werden müssen. Die Berechnung der zweiten Ableitungen wäre nicht nur kostenintensiv, sondern aufgrund von Rundungsfehlern potentiell unbrauchbar. Zudem können in der gleichen Zeit auch mehrere schnelle Iterationen der vereinfachten Variante berechnet werden. Die Vernachlässigung dieses Terms ist in der Nähe der Lösung meist unkritisch, da dort die Residuen entsprechend klein sind. Bei großer Entfernung vom Ziel wird das Ergebnis jedoch stark verfälscht.

\subsection{Levenberg-Marquardt-Verfahren}

Die Idee des Levenberg-Marquardt-Algorithmus (Levenberg, 1944; Marquardt, 1963) basiert auf der Kombination des Verfahrens des steilsten Abstiegs und dem Gauss-Newton-Verfahren in der Form:

$$
B_{k}=J_{k}^{T} J_{k}+\lambda^{L} \cdot I, \quad \lambda^{L} \geq 0 .
$$

Entsprechend ergibt sich der Levenberg-Marquardt-Korrekturschritt zu:

$$
d_{k}^{L M}=-\left(J_{k}^{T} J_{k}+\lambda^{L} \cdot I\right)^{-1} \nabla f\left(x_{k}\right) .
$$

Die Wahl eines großen $\lambda^{L}$ führt zu einer hohen Wichtung des Terms $\lambda^{L} \cdot I$, während $J_{k}^{T} J_{k}$ an Gewicht verliert und entspricht somit näherungsweise einem Gradienten-Korrekturschritt:

$$
d_{k}^{L M} \approx-\frac{1}{\lambda^{L}} \nabla f\left(x_{k}\right)
$$

Im Gegensatz dazu führt ein kleines $\lambda^{L}$ zu einer starken Wichtung von $J_{k}^{T} J_{k}$ bei gleichzeitig kleinem $\lambda^{L} \cdot I$ und stellt somit näherungsweise den Gauss-Newton-Korrekturschritt dar.

Um die Wahl zwischen beiden Möglichkeiten bzw. den Übergang zu automatisieren, wird ein so genannter Fortschrittsquotient (FQ) eingeführt, welcher ein Maß für die Güte des Schrittes darstellen soll. Es wird demzufolge nach einem probeweise gegangenen Schritt erst ausgewertet, ob dieser zu einer Verringerung von $f$ geführt hat, also "gut" ist, und somit 
akzeptiert wird. Andernfalls wird er ignoriert und die Parameter werden dementsprechend neu angepasst.

Der Fortschrittsquotient $\rho$ ergibt sich dabei nach

$$
\rho=\frac{f\left(x_{k}\right)-f\left(x_{k+1}\right)}{m_{k}\left(x_{k}\right)-m_{k}\left(x_{k+1}\right)}
$$

wobei mit $m_{k}(\cdot)$ abermals das quadratische Ersatzmodell an der Stelle $x_{k+1}$ mit der approximierten Hesse-Matrix $J_{k}^{T} J_{k}$ bezeichnet wird. Mittels Gl. 3.23 wird nur ein $d_{k}^{L M}$ gefunden, welches das Ersatzmodell näherungsweise löst. Es stellt sich jedoch die Frage, ob sich dadurch auch der Funktionswert $f(x)$ verringert. Gl. 3.25 beschreibt eben die reale Verbesserung im Vergleich zum durch das Ersatzmodell vorhergesagten $m_{k}$. Falls $\rho$ nahe 1 ist, stimmen Modell und Realität gut überein. Ist $\rho$ jedoch größer als 1 , bedeutet dies eine Verbesserung gegenüber der Erwartung. Bei einem Wert nahe, jedoch größer als 0 hat sich der Funktionswert zwar verbessert, jedoch nicht so gut wie erwartet. Werte kleiner als 0 kennzeichnen eine Verschlechterung.

Praktischerweise wird $\rho$ dabei in drei Bereiche unterteilt:

\begin{tabular}{|c|c|c|c|}
\hline$\rho \geq \eta_{h i}$ & guter Fortschritt & Richtung Newtonschritt & $\lambda_{k+1}^{L}=\lambda_{k}^{L} \cdot \sigma_{1}$ \\
\hline$\eta_{l o} \leq \rho<\eta_{h i}$ & mäßjiger Fortschritt & Schrittart beibehalten & $\lambda_{k+1}^{L}=\lambda_{k}^{L}$ \\
\hline$\rho<\eta_{l o}$ & schlechter Fortschritt & Richtung Gradientenschritt & $\lambda_{k+1}^{L}=\lambda_{k}^{L} \cdot \sigma_{2}$ \\
\hline
\end{tabular}

Dabei sind typisch genutzte Parametersätze: $\eta_{l o}=0.25, \eta_{h i}=0.75, \sigma_{1}=0.5$ und $\sigma_{2}=2$. Die Änderung von $\lambda^{L}$ in dieser Art ergibt sich aus den folgenden Überlegungen:

Wurde ein guter Fortschritt erzielt, wird die Annäherung an den Zielpunkt angenommen. Aufgrund der lokalen Konvergenz des Newtonverfahrens wird dementsprechend der Parameter $\lambda^{L}$ in diese Richtung angepasst.

War der Fortschritt hingegen schlecht, liegt der Schluss nahe, dass die Modellfunktion ungünstig gewählt wurde. Daher wird $\lambda^{L}$ erhöht, um den Anteil des Gradienten-Schrittes stärker zu gewichten und die Schrittweite zur verkürzen. Für ein genügend großes $\lambda^{L}$ wird demnach auf jeden Fall wieder die Bewegung in eine vielversprechende Abstiegsrichtung erwartet, wohingegen das Newtonverfahren schlechter iterieren würde.

War der Schritt mäßig gut, so ist die Wahl von $\lambda^{L}$ zweckmäßig gewesen und wird so lange beibehalten, bis sich der FQ ändert.

Der Schritt $x_{k+1}=x_{k}+d_{k}^{L M}$ wird nur verwendet, falls $\rho>0$ ist. In diesem Fall hat sich der Funktionswert $f(x)$ verringert.

\subsubsection{Levenberg-Marquardt als Trust-Region-Verfahren}

Ein Problem besteht darin, dass das quadratische Ersatzmodell (Gl. 3.15) im Allgemeinen nur innerhalb einer begrenzten Umgebung der aktuellen Entwicklungsstelle $x_{k}$ eine zuverlässige Approximation darstellt. Ohne eine geeignete Begrenzung der Schrittweite würde das Ersatzmodell in Bereichen Anwendung finden, wo es keine gute Approximation darstellt. 
Ein Trust-Region-Verfahren löst dieses Problem, indem ein Vertrauensbereich (Trust Region, TR) eingeführt wird, innerhalb dessen dem Ersatzmodell vertraut wird. Dieses Gebiet wird stellvertretend mit einem Parameter $\Delta$, dem Trust-Region-Radius, charakterisiert. Als Nebenbedingung ergibt sich demnach stets $\left\|d_{k}\right\| \leq \Delta$. Grundsätzlich wird auch bei dieser Variante ein Fortschrittsquotient FQ (wie in Gl. 3.25) gebildet, wobei jener jedoch nur Einfluss auf $\Delta$ hat. Genauer:

\begin{tabular}{|c|c|c|c|}
\hline$\rho \geq \eta_{h i}$ & guter Fortschritt & $\Delta_{k+1}=\Delta_{k} \cdot \sigma_{2}$ & $x_{k+1}=x_{k}+d_{k}$ \\
\hline$\eta_{l o} \leq \rho<\eta_{h i}$ & mäßiger Fortschritt & $\Delta_{k+1}=\Delta_{k}$ & $x_{k+1}=x_{k}+d_{k}$ \\
\hline$\rho<\eta_{l o}$ & schlechter Fortschritt & $\Delta_{k+1}=\Delta_{k} \cdot \sigma_{1}$ & $x_{k+1}=x_{k}$ \\
\hline
\end{tabular}

Im Gegensatz zum Levenberg-Marquardt-Verfahren wird hier direkt der Trust-Region-Radius vergrößert, falls ein guter Fortschritt erzielt wurde bzw. im anderen Fall verkleinert, um in einem kleineren Gebiet eine bessere Lösung finden zu können.

Folgende Aussagen sind äquivalent:

1. Der Vektor $d^{*}$ ist eine globale Lösung von:

$$
\begin{aligned}
& \min _{d} m_{k}(d)=f\left(x_{k}\right)+d_{k}^{T} \nabla f\left(x_{k}\right)+\frac{1}{2} d_{k}^{T} J_{k}^{T} J_{k} d_{k}, \\
& \text { mit } \quad d \in \mathbb{R}^{n} \quad \text { und unter } \quad\|d\| \leq \Delta .
\end{aligned}
$$

2. Es existiert ein eindeutig bestimmtes $\lambda^{L} \geq 0$, sodass gilt:

$$
\begin{aligned}
& \left(J_{k}^{T} J_{k}+\lambda^{L} \cdot I\right) d^{*}=-\nabla f\left(x_{k}\right), \\
& \lambda^{L} \geq 0, \quad\left\|d^{*}\right\| \leq \Delta, \quad \lambda^{L}\left(\left\|d^{*}\right\|-\Delta\right)=0 .
\end{aligned}
$$

Somit ist der Levenberg-Marquardt-Schritt $d_{k}^{L M}$ für ein gegebenes $\lambda^{L}$ die Lösung eines TrustRegion-Problems für einen implizit gewählten Trust-Region-Radius $\Delta_{k}=\left\|d_{k}^{L M}\right\|$. Dies kann der Komplementaritätsbeziehung aus Teil 2 entnommen werden. Falls $\lambda^{L}>0$ ist, führt der Schritt immer zum Rand der TR, bzw. wird der Radius durch den Schritt definiert. Ist $\lambda^{L}=0$, so befindet man sich innerhalb der TR, was einem gesichertem Gauss-Newton-Schritt entspricht.

Neben dem im Levenberg-Marquardt-Algorithmus implizit enthaltenen Trust-Region-Verfahren kann wahlweise auch ein weiterer externer Trust-Region-Radius gewählt werden, welcher den Levenberg-Marquardt-Korrekturschritt $d_{k}^{L M}$ zusätzlich begrenzen kann. Insbesondere bei schlechten Startwerten kann die Nutzung dieser Möglichkeit sinnvoll sein.

Die Ausführungen in diesem Abschnitt stellen einen Vorgriff auf die allgemeine Darstellung der Trust-Region-Verfahren in Kap. 4.1 dar, auf welches bezüglich weiterer Informationen zu dieser Thematik verwiesen wird.

\subsubsection{Modifikation nach Fletcher}

Fletcher sah in (Fletcher, 1971) zwei Nachteile des Levenberg-Marquardt-Verfahrens:

- Die Erhöhung bzw. Verringerung des Parameters $\lambda^{L}$ durch ein konstantes $\sigma_{1}$ und $\sigma_{2}$ ist unflexibel. Sinnvoller erscheint es, den Wachstums- bzw. Dämpfungsfaktor entsprechend der aktuellen Situation zu bestimmen. 
- Bei der Reduktion von $\lambda^{L}$ erfolgt die Näherung an 0 lediglich asymptotisch. Für einen echten Gauss-Newton-Schritt ist jedoch $\lambda^{L}=0$ nötig. Eine Alternative bestünde demnach in einer Schranke für $\lambda^{L}$, welche den Verlauf abschneidet und sofort auf 0 setzt.

Bereits Marquardt hatte die Idee, die Erhöhung bzw. Verringerung von $\lambda^{L}$ durch einen allgemeinen Parameter $\nu^{F}$ zu steuern. Um die Erhöhung zu optimieren, wird $\nu^{F}$ mittels folgender Gleichung berechnet:

$$
\nu^{F}=2+\frac{f\left(x_{k}\right)-f\left(x_{k}+d\right)}{d^{T} \nabla f\left(x_{k}\right)} .
$$

Dabei unterliegt $\nu^{F}$ der Boxbeschränkung $2 \leq \nu^{F} \leq 10$. Bei schlechtem Fortschritt $\left(\rho<\eta_{l o}\right)$ entspricht die Modifikation $\lambda_{k+1}^{L}=\lambda_{k}^{L} \cdot \nu^{F}$ für große Werte von $\lambda^{L}$ somit einer Reduzierung des Schrittes $d$ auf $\frac{1}{\nu^{F}} d$. Grund dafür ist der Übergang zu einem gestauchten Gradientenschritt entsprechend G1. 3.24.

Falls der Fortschrittsquotient $\rho$ größer als Null ist, gilt $f\left(x_{k}\right)>f\left(x_{k+1}\right)$. In diesem Fall ist $\nu^{F}$ kleiner als 2 und wird aufgrund der Boxbeschränkung auf $\nu^{F}=2$ gesetzt. Demnach wird ein Schritt, welcher Verbesserung bringt, nicht übermäßig stark geändert. Gilt hingegen $\rho<0$ und somit $f\left(x_{k}\right)<f\left(x_{k+1}\right)$, ergibt sich $\nu^{F}>2$. Je schlechter der Schritt ist, desto stärker wird $\lambda^{L}$ durch $\nu^{F}$ erhöht.

In diesem Zusammenhang kann auch das Stagnieren der Identifikation verhindert werden, falls $\rho$ die Grenze $\eta_{l o}$ unterschreitet. Die Erhöhung von $\lambda^{L}$ verursacht in einem solchen Fall für einen zu klein gewählten konstanten Faktor unnötige Iterationen. Andererseits kann ein zu groß gewählter Faktor dazu führen, dass der Schritt nicht angenommen wird. Durch die Nutzung von $\nu^{F}$ kann dies verhindert werden, da sowohl die stetige als auch die schleichende Verschlechterung durch den Parameter aufgenommen und somit $\lambda^{L}$ zielführend erhöht wird. Bei gutem Fortschritt $\left(\rho>\eta_{h i}\right)$ fällt nach Fletcher (1971) auf, dass sich bei der Nutzung von Faktoren der Form $\frac{1}{\nu^{F}}$ für sinkende $\lambda^{L}$ die Schrittlänge kaum verändert. Um die daraus folgende geringere Konvergenzrate zu vermeiden, führt er den Cut-Off-Parameter $\lambda_{C}^{L}$ ein:

$$
\lambda_{k+1}^{L}=\left\{\begin{array}{l}
\frac{\lambda_{k}^{L}}{2}, \text { falls } \frac{\lambda_{k}^{L}}{2} \geq \lambda_{C}^{L} \\
0, \text { falls } \frac{\lambda_{k}^{L}}{2}<\lambda_{C}^{L}
\end{array}\right.
$$

Für kleine Werte von $\lambda^{L}$ wird demnach sofort ein reiner Gauss-Newton-Schritt genutzt, wodurch quadratische Konvergenz sichergestellt wird.

Es stellt sich jedoch die Frage, wie $\lambda_{C}^{L}$ gewählt werden muss. Ein zu kleines $\lambda_{C}^{L}$ verhindert den gewünschten Effekt, wohingegen bei einem zu großen Wert der Gauss-Newton-Schritt potentiell zu früh gegangen wird und dadurch Oszillationen entstehen. Zur Lösung dessen wird bei einer Erhöhung von $\lambda^{L}$ der Cut-Off-Parameter $\lambda_{C}^{L}$ automatisch neu berechnet und anschließend $\lambda^{L}$ auf diesen Wert gesetzt. Für den auf diese Weise mittels $\lambda_{C}^{L}$ berechneten Schritt $d^{F}$ soll folgende Gleichung gelten:

$$
\left\|d^{F}\right\|=\frac{1}{2}\left\|d^{G N}\right\|
$$


Unter der Annahme, dass ein reiner Gauss-Newton-Schritt nur für $\lambda^{L}=0$ durchgeführt wird, soll demnach bei der Erhöhung von 0 auf $\lambda_{C}^{L}$ die Schrittlänge der Hälfte eines solchen Gauss-Newton-Schrittes entsprechen. Um dies (näherungsweise) zu erfüllen, wird der CutOff-Parameter $\lambda_{C}^{L}$ wie folgt definiert:

$$
\lambda_{C}^{L}=\frac{1}{\left\|\left(J^{T} J\right)^{-1}\right\|} .
$$

Während der Identifikation ist die Aktualisierung von $\lambda_{C}^{L}$ immer dann sinnvoll, wenn $\lambda^{L}$ von 0 aus erhöht wird. Als Startwerte werden $\lambda_{0}^{L}=0$ sowie $\lambda_{C}^{L}=0.75$ empfohlen. Somit ist das artifizielle Setzen von $\lambda^{L}$ nicht mehr notwendig, da sich dieses auf natürliche Weise entsprechend der vorgenannten Algorithmen verändert. Die Funktionsweise des LevenbergMarquardt-Fletcher-Algorithmus ist symbolisch in Bild 3.1 dargestellt.

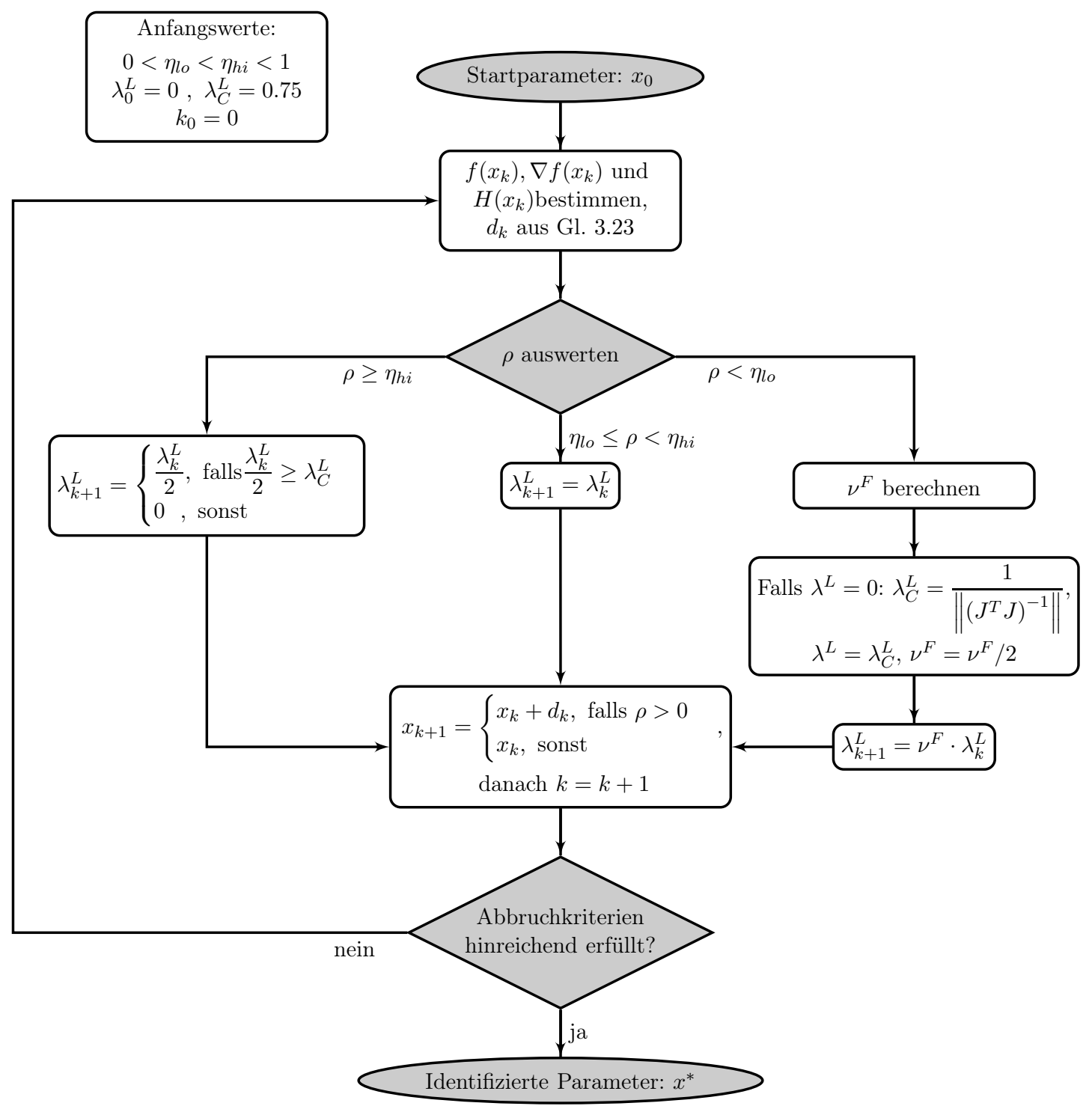

Bild 3.1: Levenberg-Marquardt-Fletcher-Algorithmus. 


\subsection{Berücksichtigung von Restriktionen}

Das in Kap. 3.6 beschriebene Verfahren zur Lösung von FQM-Aufgaben gehört zur Klasse der unrestringierten Optimierung, bei welcher Lösungen im gesamten Raum $\mathbb{R}^{n}$ zulässig sind. Bei der Identifikation von Materialparametern unterliegen deren Werte jedoch häufig Restriktionen. So führen Parameter, welche physikalisch unzulässig sind, oft zum Abbruch der FEM-Simulation. Gleiches kann auch für übermäßig große oder besonders kleine Werte zutreffen. Um dies zu vermeiden, ist für die Parameter statt $\mathbb{R}^{n}$ nur noch eine Teilmenge $U \subset \mathbb{R}^{n}$ als Lösung zugelassen. Es handelt sich somit um ein restringiertes Minimierungsproblem.

Die entsprechenden Randbedingungen werden in zwei Kategorien unterteilt:

- Ungleichungsnebenbedingung: Es liegt eine Funktion $g: \mathbb{R}^{n} \rightarrow \mathbb{R}$ mit der Bedingung $g(x) \leq 0$ vor.

- Gleichungsnebenbedingung: Es liegt eine Funktion $h: \mathbb{R}^{n} \rightarrow \mathbb{R}$ mit der Bedingung $h(x)=0$ vor.

Bei der Bestimmung von Materialparametern sind vor allem Ungleichungsnebenbedingungen relevant. Werden für einen solchen Parameter sowohl eine Ober- als auch Untergrenze definiert, wird dies als Boxbeschränkung bezeichnet und in der Form $a^{i} \leq x^{i} \leq b^{i}, a^{i}, b^{i} \in \mathbb{R}$, $i \in\{1, \ldots, m\}$ mit $m$ als Anzahl der Parameter ausgedrückt. Die Umwandlung in Ungleichungsnebenbedingungen liefert

$$
a^{i} \leq x^{i} \leq b^{i} \Longleftrightarrow\left[\begin{array}{l}
a^{i}-x^{i} \\
x^{i}-b^{i}
\end{array}\right] \leq 0 .
$$

Die Lösung restringierter Probleme ist mit dem dargestellten Levenberg-Marquardt-FletcherAlgorithmus nicht möglich. Es existiert jedoch eine Reihe anderer Verfahren zur Bearbeitung dieser Aufgaben, wobei die Klasse der SQP-Verfahren (Sequential Quadratic Programming) den derzeit erfolgreichsten Ansatz darstellt. Da die Implementierung des SQP-Ansatzes in SPC-Opt zwar weitestgehend abgeschlossen ist, jedoch noch nicht im Rahmen konkreter Aufgabenstellungen genutzt wurde, wird an dieser Stelle auf die mathematische Herleitung verzichtet. Einen Überblick bezüglich der Lösungsverfahren restringierter Optimierungsprobleme und speziell der verschiedenen SQP-Verfahren stellen z.B. Geiger und Kanzow (2002) vor.

Algorithmen wie SQP, welche speziell für restringierte Probleme entwickelt wurden, sind im Gegensatz zu unrestringierten Minimierungsverfahren deutlich aufwändiger. Im Folgenden werden verschiedene in $S P C$ - $O p t$ verfügbare Vorgehensweisen dargestellt, um mittels des Levenberg-Marquardt-Fletcher-Verfahrens auch Optimierungen der Form

$$
\min _{x \in \mathcal{X}} f(x)
$$

lösen zu können. Der Parameterraum $\mathcal{X}$ ist hierbei als Raum mit zweiseitigen Boxbeschränkungen entsprechend Gl. 3.30 gegeben und die möglichen Lösungen sollen für alle Optimierungsschritte innerhalb des zulässigen Bereiches liegen. 


\section{Spiegelung}

Ausgangspunkt der Überlegung ist, dass in einem Optimierungsschritt $k$ die neuen Lösungen $x_{k+1}=x_{k}+d_{k}$ vorliegen, jedoch mehrere Parameter außerhalb von $\mathcal{X}$ liegen können. Ohne entsprechende Überprüfung verbleiben diese außerhalb des zulässigen Raums (Bild 3.2). Für die Lösungen wird deshalb eine Korrektur der Form

$$
x_{k+1}= \begin{cases}a+\left(a-x_{k}-d_{k}\right), & \text { falls } x_{k}+d_{k}<a \\ x_{k}+d_{k}, & \text { falls } x_{k}+d_{k} \in[a, b] \\ b-\left(x_{k}+d_{k}-b\right), & \text { falls } x_{k}+d_{k}>b\end{cases}
$$

vorgenommen. Es erfolgt demnach für jeden Parameter eine Spiegelung desjenigen Schrittanteils an der Grenze $a$ bzw. $b$, welcher außerhalb des durch $a$ und $b$ begrenzten Gebietes liegt (Bild 3.3).

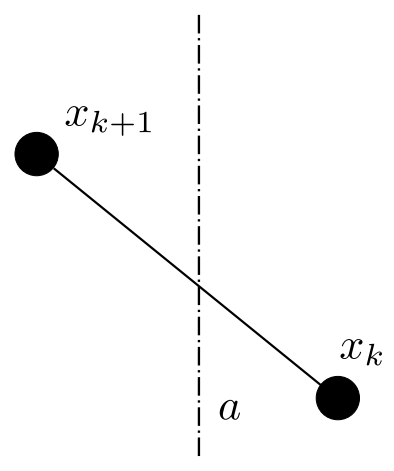

Bild 3.2: Optimierungsschritt ohne Grenzbetrachtung.

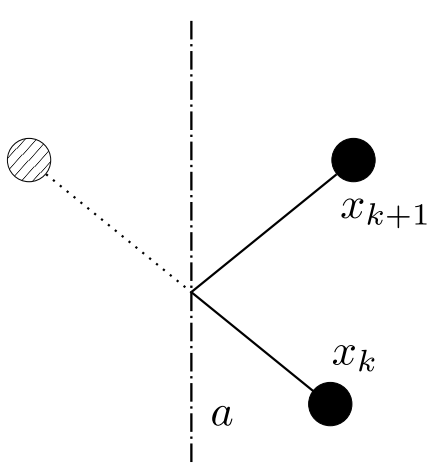

Bild 3.3: Spiegelung an Grenze $a$ der Boxbeschränkung.

Bei dieser Vorgehensweise ist jedoch schlecht abzuschätzen, inwieweit der gespiegelte Punkt eine bessere Lösung als der Ausgangspunkt darstellt bzw. ob diese Wahl günstig bezüglich der Minimierung der Optimierungsschritte ist. Im Extremfall kann die Spiegelung an einer Grenze bei besonders großer Schrittweite sogar dazu führen, dass die gegenüber liegende Grenze verletzt wird.

\section{Projektion}

Sollte eine Lösung $x_{k+1}$ außerhalb von $\mathcal{X}$ liegen, so wird diese im Gegensatz zur Spiegelung bei dieser Vorgehensweise auf die verletzte Grenze projiziert. Die Korrektur des Parametersatzes erfolgt somit entsprechend

$$
x_{k+1}= \begin{cases}a, & \text { falls } x_{k}+d_{k}<a \\ x_{k}+d_{k}, & \text { falls } x_{k}+d_{k} \in[a, b] \\ b, & \text { falls } x_{k}+d_{k}>b .\end{cases}
$$




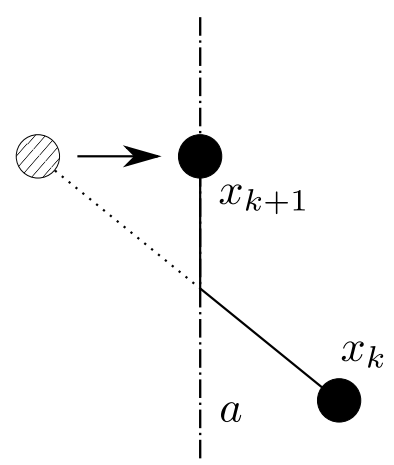

Bild 3.4: Projektion auf Grenze $a$ der Boxbeschränkung.

Da bei dieser in Bild 3.4 dargestellten Variante die neue Lösung auf der Grenze liegt, der Schrittvektor jedoch nach wie vor anhand des ursprünglichen Systems $H\left(x_{k}\right) d_{k}=-\nabla f\left(x_{k}\right)$ ermittelt wird, tritt potentiell das in Bild 3.5 verdeutlichte Verhalten auf. Angenommen wird hierbei, dass sich der optimale Parametersatz $x^{*}$ außerhalb von $\mathcal{X}$ befindet. Die dargestellten Funktionswerte entlang der Grenze verhalten sich linear. Durch die einzelnen Projektionen werden die Schrittvektoren derart verändert, dass sich die Lösungen entlang der Grenze $a$ bewegen. Dies wiederholt sich und die Lösung konvergiert zur Projektion von $x^{*}$ auf $a$. Da die tatsächlich beste Lösung auf der Grenze jedoch durch die vollständige Zielfunktion bestimmt wird, muss diese keineswegs mit der gefundenen Lösung $x_{\text {end }}$ übereinstimmen. Dies führt auf die Idee der Optimierung in Unterräumen.

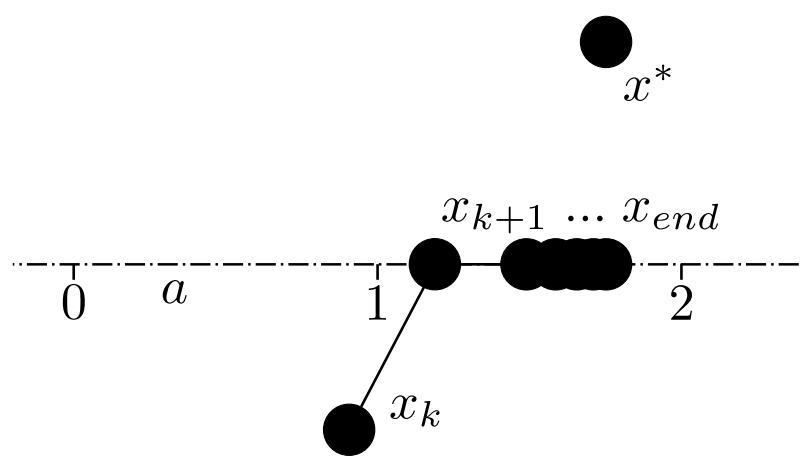

Bild 3.5: Verlauf der Optimierungsschritte bei der Projektionsmethode entlang der Grenze $a$, wobei sich der optimale Parametersatz $x^{*}$ außerhalb von $\mathcal{X}$ befindet.

\section{Unterraummethode}

Der grundlegende Unterschied gegenüber der Vorgehensweise, neue Schrittvektoren stets unter Berücksichtigung der kompletten Zielfunktion zu generieren, ist die Reduzierung des Parameterraum $\mathcal{X}$ bei Erreichen einer Grenze. Hierfür wird die Boxbeschränkung eines Parameters $i$ genau dann als "aktiv" bezeichnet, wenn für die Lösung $x_{k}$ die Gleichung $x_{k}^{i}=a^{i}$ bzw. $x_{k}^{i}=b^{i}$ gilt. Andernfalls wird sie als "inaktiv" angenommen. Diese Einordnung entscheidet, ob eine Lösung im aktuellen Optimierungsschritt modifiziert wird oder unverändert bleibt. Bei aktiver Boxbeschränkung erfolgt die Optimierung für diesen Parameter nicht im 
$m$-dimensionalen Lösungsraum, sondern nur noch innerhalb eines $(m-1)$-dimensionalen Unterraumes von $\mathcal{X}$. Dementsprechend werden die Hesse-Matrix sowie die Gradienten mittels

$$
H^{\text {red }}=A H A \quad \nabla f^{r e d}=A \nabla f
$$

transformiert, wobei $A$ eine Diagonalmatrix der Form

$$
A_{i, i}= \begin{cases}1, & \text { falls Boxbeschränkung } i \text { inaktiv } \\ 0, & \text { falls Boxbeschränkung } i \text { aktiv }\end{cases}
$$

darstellt. Somit werden diejenigen Zeilen bzw. Spalten von $H$ bzw. $\nabla f$ gestrichen, welche zu aktiven Boxbeschränkungen gehören. Wird nun das reduzierte Gleichungssystem $H^{\text {red }} d^{r e d}=-\nabla f^{r e d}$ gelöst, so verläuft $d^{\text {red }}$ nur innerhalb des Unterraumes und verletzt die Grenze nicht. Dies wird für alle Lösungen mit aktiven Boxbeschränkungen durchgeführt und so oft wiederholt, bis der resultierende Schrittvektor bei Lösung des Systems $H d=-\nabla f$ in $\mathcal{X}$ verläuft. Demnach können einzelne Richtungen unabhängig voneinander aktiv bzw. inaktiv sein.

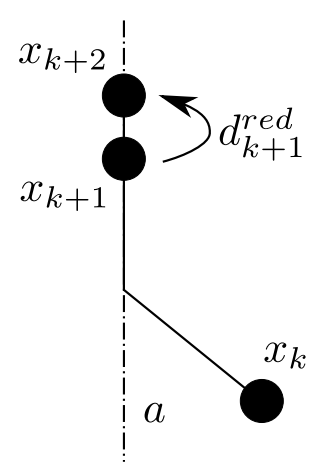

Bild 3.6: Projektion auf eine Grenze $a$ und Bestimmung von $d_{k+1}^{\text {red }}$ im darauf folgenden Schritt mittels Unterraummethode.

Eine gesonderte Betrachtung erfolgt für Punkte, bei denen alle Boxbeschränkungen aktiv sind (im Weiteren als Ecke bezeichnet). Durch die Reduzierung mittels $A$ würde $H$ in eine Nullmatrix übergehen. Da eine solche Ecke nicht zwangsläufig den Abbruch der Optimierung bedeutet, muss es möglich sein, diese wieder verlassen zu können. Dies wird durch den Vergleich der Anteile des Gradienten in der Ecke gewährleistet, wobei der Anteil mit dem größten Abstieg in Richtung von $\mathcal{X}$ gewählt und ein entsprechender Schrittvektor künstlich generiert wird. Ist in einer Ecke keine solche Abstiegsrichtung vorhanden, wird die Optimierung abgebrochen, da keine Verbesserung auffindbar ist.

Bei der Unterraummethode wird demnach zuerst immer geprüft, ob ein berechneter Schritt die Boxbeschränkung verletzt. Ist dies der Fall und befindet sich der Ausgangspunkt nicht auf der betrachteten Grenze, wird die neue Lösung auf diese projiziert. Liegt der Ausgangspunkt hingegen bereits auf dieser Grenze, so wird bei Verletzung der Boxbeschränkung der zulässige Raum $\mathcal{X}$ entsprechend reduziert und das reduzierte System $H^{\text {red }} d^{\text {red }}=-\nabla f^{\text {red }} \mathrm{im}$ Unterraum optimiert. 


\subsection{Abbruchbedingungen}

Einen wichtigen Aspekt der numerischen Optimierung stellt die Wahl der Abbruchbedingungen dar. Diese sind notwendig, da die perfekte Übereinstimmung zwischen Modell und Experiment praktisch nicht möglich ist, die Anzahl der Iterationen jedoch sinnvoll begrenzt werden muss. Ist bereits ein einzelner Optimierungsschritt sehr zeitaufwändig, wie z.B. bei der Identifikation anhand bauteilnaher Probekörper (Kap. 6.7), hat die geschickte Definition von Abbruchkriterien einen besonders hohen Stellenwert.

Grundsätzlich ist der Abbruch einer Optimierung immer dann sinnvoll, wenn die Abweichung zwischen aktueller und idealer Lösung hinreichend klein ist. Ausgehend vom theoretischen Minimum $\nabla f\left(x^{*}\right)=0$ wird deshalb gewöhnlich eine Schranke $\varepsilon^{A}$ definiert, mit welcher die Abbruchbedingung

$$
\left\|\nabla f\left(x_{k}\right)\right\| \leq \varepsilon^{A}
$$

gebildet wird. Ergänzend werden in Gill et al. (1981) weitere Abbruchkriterien vorgeschlagen, welche sich auch auf die Änderung der Funktionswerte $\left\|f\left(x_{k-1}\right)-f\left(x_{k}\right)\right\|$ bzw. der Lösungen $\left\|x_{k-1}-x_{k}\right\|$ beziehen und dabei sowohl absolute als auch relative Änderungen beinhalten.

Bei der Wahl von $\varepsilon^{A}$ ist zu beachten, dass aufgrund der begrenzten Genauigkeit unterhalb eines kritischen Wertes die Ergebnisse unsicher bzw. fehlerbehaftet sind. Ergänzend zu den Abbruchbedingungen ist es sinnvoll, die Anzahl der Optimierungsschritte durch eine Obergrenze zu beschränken.

Da zu Beginn der Optimierung die Wahl des Abbruchkriteriums und der Abbruchgrenze schwierig ist, können in $S P C$-Opt mehrere gewichtete Abbruchbedingungen zu einem Kriterium $\bar{E}^{A}$ zusammengefasst werden. Durch die Einführung einer Wichtung können Abbruchbedingungen, welche für den Anwender eine höhere Priorität besitzen, auch in höherem Maße berücksichtigt werden. Für eine solche Teilbedingung $E_{j}^{A}$ sind folgende Größen relevant:

$$
\begin{array}{ll}
a_{j}(x) & \text { Bildungsvorschrift der Abbruchbedingung } \\
\varepsilon_{j}^{A} & \text { Abbruchschranke } \\
w_{j} & \text { Wichtungsfaktor } .
\end{array}
$$

Der Anteil einer Teilbedingung bezüglich $\bar{E}^{A}$ ergibt sich für eine Lösung $x$ somit zu

$$
E_{j}^{A}(x)=w_{j} \chi_{j}(x)
$$

mit der charakteristischen Funktion

$$
\chi_{j}(x)=\left\{\begin{array}{l}
1, \text { falls } a_{j}(x) \leq \varepsilon_{j}^{A} . \\
0, \text { sonst }
\end{array}\right.
$$

Die Summe aller Abbruchbedingungen für eine Lösung $x$ besitzt demzufolge die Form

$$
\bar{E}^{A}(x)=\sum_{j=1}^{n_{E}} E_{j}^{A}(x)=\sum_{j=1}^{n_{E}} w_{j} \chi_{j}(x),
$$


mit $n_{E}$ als Anzahl der Abbruchbedingungen. Überschreitet diese Summe eine definierte Grenze $\bar{\varepsilon}^{A}$, so wird die Optimierung abgebrochen. Dabei sollte $\bar{\varepsilon}^{A}$ zwischen den Werten 0 (immer erfüllt) und $\sum_{j=1}^{n_{E}} w_{j}$ (alle Bedingungen müssen erfüllt sein) verlaufen. Es ist demnach nicht notwendig, dass die Schranken sämtlicher Teilbedingungen unterschritten werden müssen.

Folgende Abbruchbedingungen sind in SPC-Opt implementiert und können für eine Lösung $x_{k}$ ausgewertet werden:

\section{Schrittweite}

Gilt als erfüllt, falls die Differenz der aktuellen und der letzten akzeptierten Lösung $x_{\mathrm{A}}$ klein genug ist.

$$
a_{j}\left(x_{k}\right)=\left\|x_{k}-x_{\mathrm{A}}\right\|_{2}
$$

\section{Relative Schrittweite}

Gilt als erfüllt, falls die maximale relative Schrittweite aller Koordinaten $i$ zwischen der aktuellen und der letzten akzeptierten Lösung klein genug ist.

$$
\begin{aligned}
& h^{i}\left(x_{k}\right)=\frac{\left|x_{k}^{i}-x_{\mathrm{A}}^{i}\right|}{x_{\mathrm{A}}^{i}}, \forall i \in\{1, \ldots, n\} \\
& a_{j}\left(x_{k}\right)=\max _{i \in\{1, \ldots, n\}} h^{i}\left(x_{k}\right)
\end{aligned}
$$

\section{Funktionswert}

Gilt als erfüllt, falls der Funktionswert klein genug ist (bei FQM-Problemen idealer Weise gleich 0).

$$
a_{j}\left(x_{k}\right)=f\left(x_{k}\right)
$$

\section{Differenz der Funktionswerte}

Gilt als erfüllt, falls die Änderung des Funktionswertes klein genug ist.

$$
a_{j}\left(x_{k}\right)=\left|f\left(x_{k-1}\right)-f\left(x_{k}\right)\right|
$$

\section{Gradient}

Gilt als erfüllt, falls die Länge des Gradienten klein genug ist.

$$
a_{j}\left(x_{k}\right)=\left\|\nabla f\left(x_{k}\right)\right\|_{2}
$$

\section{[NA]-Schrittweite}

Gilt als erfüllt, falls der Schrittvektor zwischen zwei aufeinander folgenden nicht akzeptierten Punkten klein genug ist.

$$
a_{j}\left(x_{k}\right)=\left\|x_{k-1}-x_{k}\right\|_{2}, \text { falls } x_{k} \text { und } x_{k-1} \text { nicht akzeptiert }
$$

Die Erfüllung der [NA]-Schrittweiten-Bedingung ist ein Indiz dafür, dass kein neuer akzeptabler Parametersatz gefunden wird. Es ist deshalb sinnvoll, die Wichtung dieser Bedingung bei Bildung von $\bar{E}^{A}$ (Gl. 3.39) größer als die der übrigen Abbruchbedingungen zu wählen. 
Zwar ist die Implementierung weiterer Abbruchkriterien in SPC-Opt problemlos möglich, jedoch war dies bei der Bearbeitung der bisherigen Aufgaben nicht notwendig.

\subsection{Parameterschätzung}

Unabhängig vom gewählten Verfahren sind bei der Anpassung eines Modells an entsprechende Versuche die zugehörigen Messungen naturgemäß mit Messfehlern verbunden. Somit stellt sich die Frage nach der Vertrauenswürdigkeit der identifizierten Parameter, auch als Konfidenz bezüglich der Messgenauigkeit bezeichnet. Dies äußert sich bei der Lösung von FQM-Problemen wie der Identifikation von Materialparametern dadurch, dass in der Nähe des Minimums der Zielfunktion für verschiedene Parameterkonstellationen nur gering voneinander abweichende Funktionswerte auftreten.

Insbesondere bei der Identifikation von Materialparametern kann beobachtet werden, dass teilweise eine Korrelation zwischen diesen auftritt. Dies ist der Fall, wenn verschiedene Parameter in der Lage sind, in gleicher Weise physikalische Effekte der Versuche darzustellen. Bezogen auf die Zielfunktion manifestieren sich diese Abhängigkeiten als lange, schmale Täler.

Praktische Anwendungen der Parameterschätzung finden sich u.a. in den Arbeiten von Thielecke (1997), Benedix (2000) und Lindner (2010).

Zur Bewertung des Einflusses stochastischer Effekte bei Messungen werden gewöhnlich Schätzfunktionen genutzt (Sorenson, 1980; Bos, 2007). Bezogen auf die Parameteridentifikation besteht im Allgemeinen die Grundannahme darin, dass das Modell $M$ korrekt ist, die fehlerbehafteten gemessenen Größen $\bar{y}$ und die von $p$ abhängigen Modellgrößen $y$ nur geringe Unterschiede aufweisen und die Parameter $p$ fehlerfrei sind - das Modell demnach frei von stochastischen Einflüssen ist. Zur Parameterschätzung werden vor allem die MaximumLikehood-Methode sowie die Methode der kleinsten Quadrate genutzt (Beck und Arnold, 1977). Da letztere keine zusätzlichen Informationen bezüglich der Messfehler benötigt und dies für die meisten Probleme der Parameteridentifikation notwendig ist, erfolgen die weiteren Betrachtungen auf Basis dieser Methode. Weiterhin wird angenommen, dass die Messfehler voneinander unabhängig sowie nicht systematisch sind.

Auf dieser Basis wird zunächst die Varianz $\sigma^{2}$ bestimmt, welche näherungsweise der Stichprobenvarianz $s^{2}$ entspricht (Beck und Arnold, 1977):

$$
s^{2}=\frac{\left(r^{T}\left(x^{*}\right) r\left(x^{*}\right)\right)}{m-n_{p}} .
$$

Hierbei stellt $r\left(x^{*}\right)$ den Residuenvektor im Lösungspunkt, $m$ die Anzahl der Messwerte und $n_{p}$ die Anzahl der freien Parameter dar. Auf Basis von Gl. 3.46 lässt sich mit Hilfe der Jacobimatrix für die Lösung des linearisierten Modells an der Stelle $x^{*}$ die Kovarianzmatrix $P$ berechnen:

$$
P=\operatorname{cov}\left(x^{*}\right) \approx \sigma^{2}\left(J\left(x^{*}\right)^{T} J\left(x^{*}\right)\right)^{-1} .
$$

Die Diagonalelemente von $P$ stellen demnach Schätzwerte für die Varianzen der entsprechenden Parameter dar. Werden mehrere Versuche mit unterschiedlichen Vergleichsgrößen 
oder Messfehlern durchgeführt (vergl. Kap. 3.2), sind die Fehler von $\sigma^{2}$ potentiell nicht mehr konstant bzw. können untereinander korrelieren. In diesem Fall müssen zunächst die verschiedenen Vergleichswerte auf dimensionslose Größen normiert und eine darauf bezogene Matrix $\Omega$ in den Gl. 3.46 und 3.47 berücksichtigt werden. Diese ergeben sich somit zu:

$$
s^{2}=\frac{\left(r^{T}\left(x^{*}\right) \Omega^{-1} r\left(x^{*}\right)\right)}{m-n_{p}}
$$

bzw.

$$
P=\operatorname{cov}\left(x^{*}\right) \approx \sigma^{2}\left(J\left(x^{*}\right)^{T} \Omega^{-1} J\left(x^{*}\right)\right)^{-1} .
$$

Es lässt sich nunmehr (bei nichtlinearen Modellen näherungsweise) das Konfidenzintervall berechnen, dessen Grenzen den Vertrauensbereich im Parameterraum einhüllen:

$$
\operatorname{konf}\left(x_{i}\right)=\left[x_{i}^{*}-\sqrt{P_{i i}} ; x_{i}^{*}+\sqrt{P_{i i}}\right], \forall i \in\left\{1, \ldots, n_{p}\right\} .
$$

Grundsätzlich wird ein möglichst kleines Konfidenzintervall angestrebt. Aus diesem Grund sollten die Messungen sorgfältig durchgeführt werden, um die entsprechenden stochastischen Messfehler möglichst klein zu halten. Zudem bietet sich die mehrfache Wiederholung der Messungen mit anschließender Mittlung an.

Weiterhin kann die Korrelationsmatrix $K$ entsprechend

$$
K_{i j}=\frac{P_{i j}}{\sqrt{P_{i i} P_{j j}}}
$$

bestimmt werden, wobei $K_{i i}=1$ gilt. Die Nebendiagonalelemente der Korrelationsmatrix beschreiben die Korrelation der Parameter untereinander und werden durch das Intervall $[-1 ; 1]$ begrenzt. Werte nahe 1 bzw. -1 weisen hierbei auf eine hohe Korrelation der entsprechenden Parameter hin. Bei der Materialparameteridentifikation kann dies z.B. an der mathematischen Form des Stoffgesetzes, wie bei der Identifikation der OGDEN-Parameter in Kap. 6.6.4 dargestellt, liegen. Eine weitere Ursache ist der Mangel an Informationen bezüglich einzelner Parameter, welcher wiederum häufig die Folge von zu wenigen oder ungeschickt gewählten Versuchen ist. Es ist deshalb sinnvoll, bereits bei der Auswahl der für die Identifikation verwendeten Experimente deren Aussagekraft bezüglich der Materialparameter zu berücksichtigen. Ebenso gilt dies in Fällen, wenn zur Abbildung vorhandener Messdaten aus Versuchen ein entsprechendes Modell, z.B. ein Stoffgesetz, ausgewählt oder entwickelt werden soll.

Die mathematische Betrachtung des Einflusses von Parametern auf das Modell wurde bislang noch nicht in SPC-Opt implementiert. Im Hinblick auf Problemstellungen der Parameteridentifikation und Formoptimierung, welche mit dem Programmpaket bearbeitet wurden, erscheint die entsprechende Erweiterung des Funktionsumfangs aufgrund der nachfolgenden Ausführungen notwendig.

Eine exakte Definition der Sensitivität ist aufgrund des qualitativen Charakters schwierig. Im Allgemeinen dient sie als Maß zur Bewertung des Einflusses der Parameter $x_{i}$ auf die Ausgabegrößen $y_{j}$. Diese werden als sensitiv gegenüber einem Parameter bezeichnet, wenn 
bereits eine kleine Änderung von $x_{i}$ zu einer großen Änderung von $y_{j}$ führt. Dabei wird angenommen, dass neben den Parametern keine weiteren Einflüsse auf die Ausgabegrößen wirken - das System demnach deterministisch ist. Äußere stochastisch verteilte Einflussgrößen dürfen in diesem Kontext existieren, da der Erwartungswert bei einer genügend hohen Anzahl Wiederholungen unverändert bleibt. Die Kenntnis bezüglich des Einflusses von Parametern ist vor allem deshalb interessant, weil dadurch diejenigen mit einem kleinen Einfluss auf den Zielfunktionswert bei der Optimierung vernachlässigt werden können und somit die Dimension des Parameterraumes verringert werden kann. Beispielhaft seien drei konkrete Anwendungsfälle genannt:

\section{Parameteridentifikation}

Bei der in Kap. 6 dargestellten Stoffgesetzanpassung werden unter anderem die acht Materialparameter des von Ihlemann (2003) entwickelten MoRPH-Stoffgesetzes identifiziert. Entsprechend der Ausführungen in Kap. 7.7 seiner Arbeit kann die Zahl der Parameter durch Vernachlässigung des Exponentialteils der Hüllspannungen auf fünf gesenkt werden. Dies ist genau dann sinnvoll, wenn in den zur Anpassung genutzten Experimenten der exponentielle Charakter des Elastomers aufgrund zu geringer Deformationen kaum sichtbar ist oder grundsätzlich nur eine schwache Ausprägung besitzt. Durch eine entsprechende Sensitivitätsanalyse könnte dies quantifiziert werden und als Entscheidungsgrundlage bezüglich der gewählten Form des Stoffgesetzes dienen.

\section{Entwicklung eines Reibgesetzes}

In der Arbeit von Nostitz (2014) wird mit Hilfe eines 3-Skalen-Modells ein in FEMProgrammen nutzbares Reibgesetz erarbeitet, dessen Basis die infolge zwischenatomarer Kräfte auftretende Adhäsion zweier Körper darstellt. Dabei ist es notwendig, die Wirkung und Einflüsse der Adhäsionskennwerte und weiterer charakteristischer Größen der verschiedenen Skalen (z.B. die Oberflächenrauigkeit) zu untersuchen und bewerten. Auf diese Weise ist es möglich, ein für die jeweilige Skala geeignetes Reibmodell zu entwickeln. Eine Sensitivitätsanalyse ist deshalb unerlässlich und Teil der Arbeit.

\section{Formoptimeriung}

Beim Strangfließpressen, einem Massivumformverfahren, beeinflusst die Kontur der Werkzeugschulter maßgeblich die benötigte Umformkraft. Ziel ist es, die Kontur derart zu gestalten, dass die Umformkraft minimal wird. Dies erfolgt in (Schellenberg et al., 2010) durch Lösung einer entsprechenden Optimierungsaufgabe, wobei die rotationssymmetrische Werkzeugkontur mittels NURBS (Non-Uniform Rational B-Splines) abgebildet wird und die Koordinaten der zugehörigen De-Boor-Punkte in radialer Richtung die zu optimierenden Parameter darstellen. Die Wahl der Anzahl dieser Kontrollpunkte sowie die anfängliche Positionierung ist dabei willkürlich und basiert auf Erfahrungswerten. Zwar kann das Optimierungsproblem zufriedenstellend gelöst werden, jedoch würde eine Sensitivitätsanalyse zeigen, ob vernachlässigbare De-Boor-Punkte existieren oder die Positionierung in Strangrichtung ungünstig ist.

Da in der Praxis keine Kenntnis der Zielfunktionslandschaft vorliegt, der Berechnungsaufwand einer großen Menge Funktionswerte zur Approximation jedoch meist zu groß ist, kann 
keine exakte Sensitivitätsanalyse durchgeführt werden. Einen Ausweg stellen vereinfachte Verfahren dar, bei welchen die Funktionslandschaft nur grob approximiert wird bzw. statistische Methoden zur Auswertung genutzt werden. Da eine Vielzahl solcher Verfahren für unterschiedliche Anwendungsbereiche existiert, sei stellvertretend auf die Arbeit von Fiacco (1983) sowie den Überblick in Saltelli et al. (2008) verwiesen.

\subsection{Formidentifikation}

Ziel einer Materialparameteridentifikation ist die Ermittlung desjenigen Parametersatzes, für welchen die einbezogenen Experimente am besten mit den zugehörigen Simulationen übereinstimmen. Die Formidentifikation grenzt sich insoweit davon ab, dass geometrische Kenngrößen gesucht werden, für welche die Abweichung zwischen Experiment und Simulation minimal wird. In beiden Fällen erfolgt die Formulierung einer Zielfunktion, welche auf einer Fehlerquadratsumme basiert und deren Minimum gesucht wird. Da die Parameter im Optimierungsverfahren abstrakte Größen sind, gibt es aus mathematischer Sicht keine formalen Unterschiede zwischen der Identifikation von Materialparametern und Formparametern.

Liegt ein FEM-Netz in parametrisierter Form vor, können die Steuervariablen direkt für die Identifikation genutzt werden. Der Arbeitsaufwand für den Anwender ist dadurch sehr gering, was z.B. bei der Festlegung des Probekörper-Designs in Kap. 6.7.1 der Fall ist. Andernfalls erfordert die Formidentifikation bezüglich der Implementierung in SPC-Opt hingegen eine deutliche Erweiterung der Funktionalität. Dies bezieht sich vor allem auf die Beschreibung der geometrischen Konturen des Modells sowie die automatische Anpassung des FEM-Netzes bei Änderung der Formparameter (Kap. 2.5). Zur Veranschaulichung der entsprechenden Vorgehensweise bei einer Formidentifikation wird diese anhand des nachfolgenden Beispiels eines gestauchten Zylinders dargelegt.

\section{Beispiel Stauchversuch}

Bei der Bestimmung von Fließkurven für große Verformungen wird häufig der Stauchversuch eingesetzt. Infolge der immer vorhandenen Reibung kommt es im Verlauf des Experiments zu einer Ausbauchung der Probe. Darauf basierend wird ein akademisches Beispiel zur Formidentifikation abgeleitet. Das Ziel besteht darin, eine Probenform für den Ausgangszustand (Bild 3.7) so zu bestimmen, dass der Probekörper bei einer vorgegebenen Stauchung möglichst zylindrisch wird. Für die FEM-Simulation kommt das hauseigene FEM-Programm $S P C-P M 2 A d N l$ zum Einsatz. Da in dessen Kontaktalgorithmus kein Reibmodell zur Verfügung steht, mit welchem sich der Wechsel von Haft- zu Gleitreibung modellieren lässt, wird vollständiges Haften am Stempel angenommen. In der Berechnung wird elastisch-plastisches Materialverhalten mit isotroper Verfestigung nach Ulbricht und Röhle (1975) verwendet.

In Vorbereitung der Identifikation wird die Randkontur $\Omega$ des Probekörpers, deren Kontur sich durch $n$ auf dem Rand befindliche FE-Knoten ergibt, durch NURBS approximiert. Die horizontalen Koordinaten der zugehörigen Kontrollpunkte stellen die zu identifizierenden Designvariablen $\underline{p}$ dar. Die Zielfunktion $\Phi$ wird entsprechend 


$$
\Phi(\underline{p})=\sum_{i=0}^{n}\left[A\left(\underline{x}_{i}\left(\underline{X}_{i}, t=t_{\text {end }}\right), \Gamma\right)\right]^{2} \rightarrow \min _{p}
$$

gebildet, wobei $A$ den waagerechten Abstand zwischen der Zielform $\Gamma$ (im Meridianschnitt eine senkrechte Gerade) und den Knoten $\underline{X}_{i}$ auf der Randkontur $\Omega$ verkörpert. Durch die Identifikation sollen die Formparameter derart bestimmt werden, dass bei einer Stauchung um 10\% (zum Zeitpunkt $t=t_{\text {end }}$ ) die zugehörige Randkontur möglichst genau mit $\Gamma$ übereinstimmt. Bei der angegebenen Lösung wurden 4 Designvariablen verwendet.

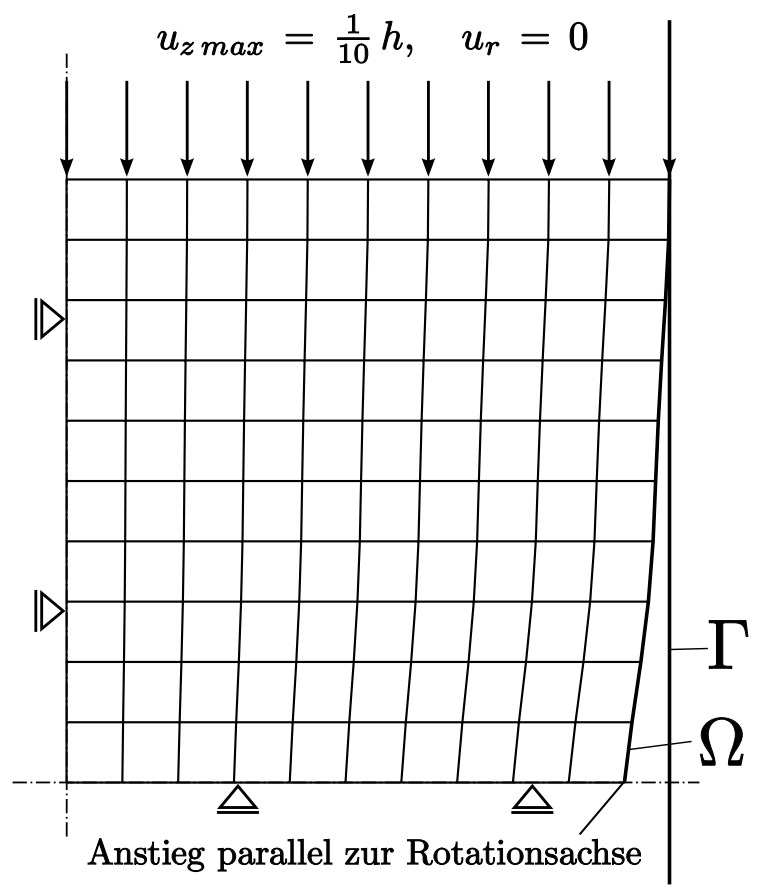

Bild 3.7: Rotationssymmetrische Stauchprobe (ein Viertel des Meridianschnitts), Vernetzung und Randbedingungen, Zustand für $\underline{x}_{0}$.
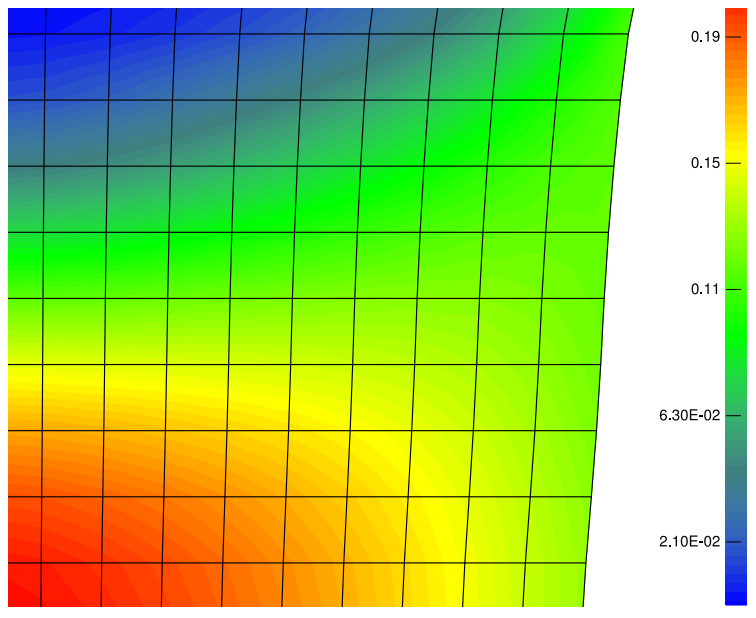

Bild 3.8: Plastische Vergleichsdehnung $\varepsilon_{e q}^{p}$ für $p_{\text {end }}$, dargestellt im Netz des undeformierten $\mathrm{Zu}-$ stands.

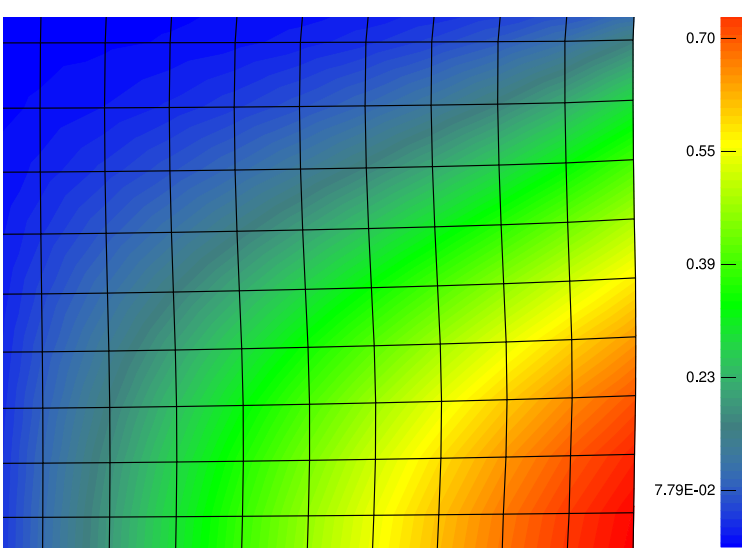

Bild 3.9: Radiale Verschiebung $u_{r}$ für $p_{\text {end }}$, dargestellt im Netz des deformierten Zustands. 
Die nach 6 Optimierungsschritten ermittelten Designvariablen $\underline{p}=\underline{p}_{\text {end }}$ führen, wie in Bild 3.9 dargestellt, nach der Stauchung zu einer annähernd zylindrischen Form der Probe.

Es ist anzumerken, dass die Genauigkeit der Übereinstimmung mit der Zielform maßgeblich durch die Anzahl der Designvariablen beeinflusst wird. Bei Verwendung von wenigen Formparametern ist die Variationsmöglichkeit der Randkontur $\Omega$ beschränkt und damit eine exakte zylindrische Form nicht erreichbar. Eine Vergrößerung der Zahl wirkt dem zwar entgegen, jedoch ist dies mit einem deutlich schlechteren Konvergenzverhalten des Optimierers verbunden. Die Ursache dafür ist, dass mit einer steigenden Zahl von Kontrollpunkten auch stärkere Korrelationen zwischen den entsprechenden Designvariablen auftreten. Zudem sinkt die Wahrscheinlichkeit, dass die gefundene Lösung auch dem globalen Minimum der Zielfunktion entspricht. In diesem Fall wäre eine Kontrolle durch die Nutzung einer Startparametervariation entsprechend Kap. 5 zu erwägen. 


\section{Formoptimierung}

Das Ziel einer Parameteridentifikation besteht darin, auf Basis optimal gewählter Parameter die Differenz zwischen experimentellen Daten und entsprechenden Simulationsergebnissen zu minimieren. Dabei ist es unerheblich, ob es sich um Form- oder Materialparameter handelt. Eine sehr vorteilhafte Eigenschaft der zugehörigen Zielfunktionen ist, dass sich diese auf ein Problem der kleinsten Fehlerquadrate (Gl. 3.5) zurückführen lässt. Aufgaben dieser Art lassen sich, wie in Kap. 3.6 beschrieben, sehr elegant mit dem LMF-Verfahren lösen. Aus mathematischer Sicht gehört dieses zu den Trust-Region-Verfahren, stellt jedoch einen Sonderfall dar. Der Grund dafür ist die Tatsache, dass bei der Approximation der Hesse-Matrix die Terme zweiter Ordnung vernachlässigt werden (vgl. Gl. 3.21), deren Bestimmung sonst sehr schwierig ist.

Im Gegensatz zur Identifikation von Parametern ist bei einer Formoptimierung nicht die Anpassung an bereits vorhandene Messdaten oder Konturen von Interesse. Vielmehr soll die Gestalt eines Bauteils derart verändert werden, dass das definierte Optimierungsziel durch Lösung des entsprechenden Optimierungsproblems bestmöglich erreicht wird. Ein typisches Optimierungsziel stellt die Minimierung oder Maximierung charakteristischer Größen wie Gewicht, Umformarbeit, Bauteilsteifigkeit oder Spannungen im Bauteil dar. Da, analog zur Formidentifikation, während der Optimierung eine oder mehrere geometrische Merkmale (meist Randkonturen) gezielt verändert werden, müssen diese in geeigneter Form parametrisiert werden (Kap. 2.5) . Die entsprechenden Formparameter (Designvariablen) stellen die zu optimierenden Parameter der Zielfunktion dar.

Bei Aufgaben der Formoptimierung ist es nicht möglich, diese auf ein FQM-Problem zurückzuführen. Somit kann auch das in Kap. 3.6 beschriebene LMF-Verfahren nicht verwendet werden. Um Aufgaben dieser Art dennoch lösen zu können, empfiehlt sich die Betrachtung von Trust-Region-Verfahren im Allgemeinen und in diesem Zusammenhang die Nutzung effizienter Algorithmen zur Approximation der Hesse-Matrix. Hierbei ist anzumerken, dass stochastische Verfahren bewusst nicht berücksichtigt werden, da sie eine große Menge Funktionsauswertungen erfordern und deren Berechnung gewöhnlich mit einem hohen numerischen Aufwand verbunden ist.

\subsection{Trust-Region-Verfahren}

Als Einstiegspunkt dient die Idee der Einführung einer Modellfunktion $m_{k}$ an der aktuellen Entwicklungsstelle $x_{k}$, wie sie bereits in Kap. 3 entsprechend Gl. 3.13 erläutert wurde. Die Übereinstimmung der tatsächlichen Funktion $f$ mit dem lokalen Ersatzmodell ist verfahrensbedingt umso schlechter, je weiter weg von der Entwicklungsstelle sie berechnet wird. Aus diesem Grund wird das Minimum von $m_{k}$ nur in einem definierten Vertrauensbereich (trust 
region) um $x_{k}$ gesucht, in welchem die Differenz von $f$ und $m_{k}$ als hinreichend klein angenommen wird. In Bild 4.1 ist das Prinzip des Trust-Region-Verfahrens für ein zweidimensionales Problem dargestellt.

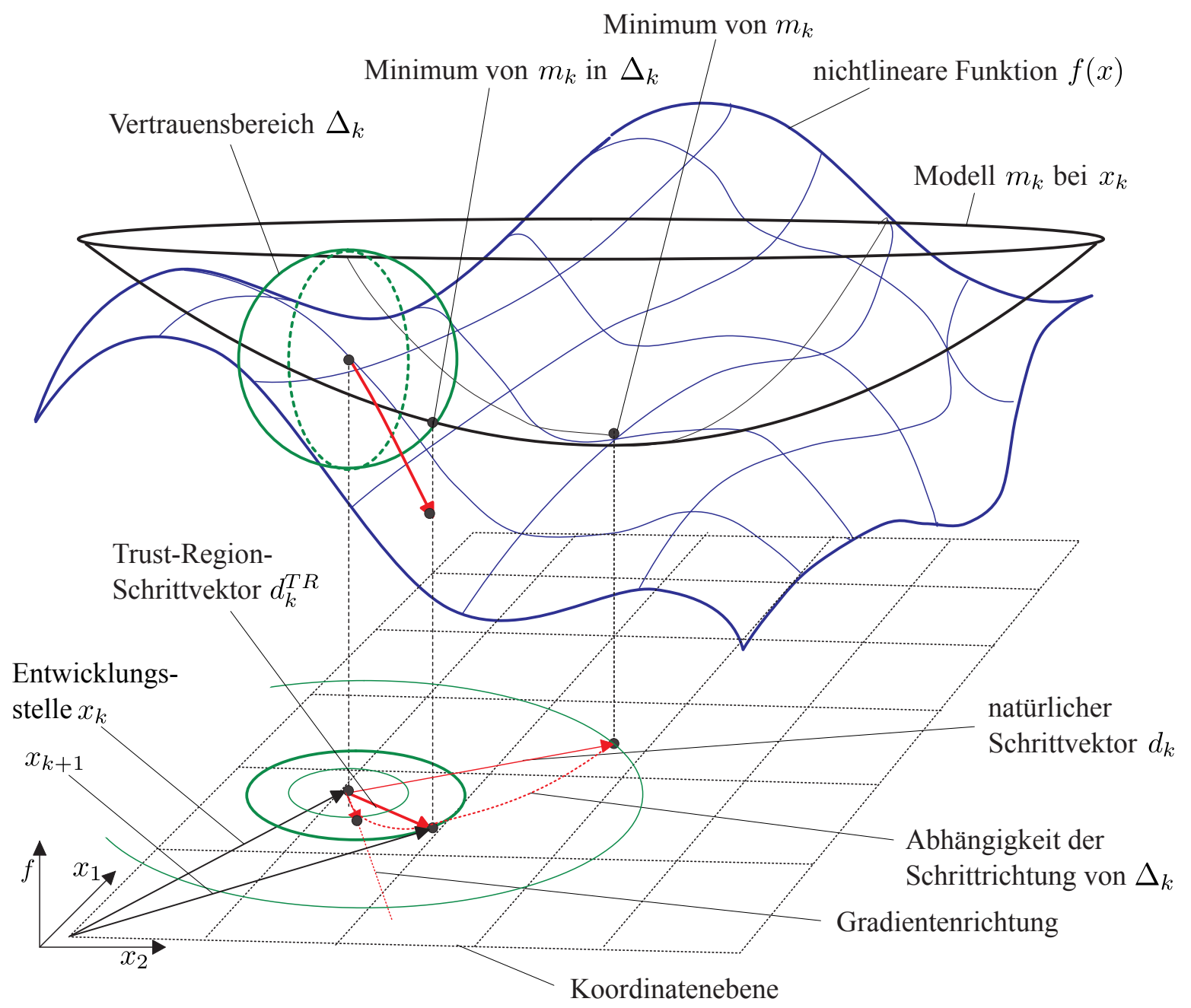

Bild 4.1: Funktionsweise des Trust-Region-Verfahrens.*

Die Schwierigkeit besteht darin, einen geeigneten Vertrauensbereich $\Delta$ an der aktuellen Entwicklungsstelle $x_{k}$ festzulegen. Wird dieser zu klein gewählt, ist die Schrittweite in Richtung des Minimums der Zielfunktion sehr gering und die Zahl der notwendigen Optimierungsschritte steigt. Ist der Wert von $\Delta$ jedoch zu groß, kann die neue Entwicklungsstelle $x_{k+1}$ sogar weiter vom Minimum der Zielfunktion $x^{*}$ entfernt sein als $x_{k}$, falls $m_{k}$ die Zielfunktion in Richtung $x^{*}$ nur schlecht abbildet. Aus diesem Grund wird der Vertrauensbereich in jedem Optimierungsschritt geprüft und bei guter Übereinstimmung von $m_{k}\left(x_{k}\right)$ mit $f\left(x_{k}\right)$ erhöht. Bei einem fehlgeschlagenen Schritt hingegen wird $\Delta$ verkleinert, was meist auch eine Änderung der Schrittrichtung zur Folge hat (Nocedal und Wright (1999), Kap. 4). Je kleiner der Trust-Region-Radius wird, desto mehr tendiert diese von der natürlichen Schrittrichtung weg zur Gradientenrichtung (vgl. Bild 4.1).

${ }^{*}$ Darstellung entstammt (Clausner, 2007$)$ 
Das Anwenden des Trust-Region-Korrekturvektors $d_{k}^{T R}$ auf Gl. 3.8 und Gl. 3.15 führt zu

$$
m_{k}\left(d_{k}^{T R}\right)=f\left(x_{k}\right)+\left(d_{k}^{T R}\right)^{T} \nabla f\left(x_{k}\right)+\frac{1}{2}\left(d_{k}^{T R}\right)^{T} \nabla^{2} f\left(x_{k}\right) d_{k}^{T R}
$$

unter der Bedingung, dass die Suche nach dem Minimum dieser Funktion auf den Vertrauensbereich beschränkt ist:

$$
\min _{d_{k}^{T R}} m_{k}\left(d_{k}^{T R}\right) ;\left\|D d_{k}^{T R}\right\| \leq \Delta_{k}
$$

Da Trust-Region-Verfahren, im Gegensatz zu Line-Search-Verfahren, sehr empfindlich auf unterschiedliche Skalierungen der Komponenten von $x$ reagieren, enthält Gl. 4.2 die Skalierungsmatrix $D$. Schlecht skalierte Probleme treten beispielsweise auf, wenn die Zielfunktion Parameter mit unterschiedlichen physikalischen Bedeutungen enthält. Dies führt dazu, dass die Zielfunktion sehr unterschiedlich sensitiv auf Störungen der verschiedenen Parameter reagiert. Auch die Topologie der Zielfunktion bildet dies ab, da sie in diesem Fall lange, schmale Täler enthält, innerhalb derer sich die Lösung das Minimum befindet. Durch eine geschickte Wahl von $D$ kann das Verfahren deutlich verbessert werden (Rao, 2009). In $S P C$ Opt stehen verschiedene Skalierungsverfahren zur Verfügung, von denen sich für viele Problemstellungen die Skalierung

$$
D_{i i}=\sqrt{\left\|\left(\nabla^{2} f\left(x_{k}\right)\right)_{i i}\right\|} \text { bzw. } \quad D_{i i}=\sqrt{\left\|\left(B_{k}\right)_{i i}\right\|}
$$

bewährt hat. Eine pauschale Aussage, welches Verfahren am besten geeignet ist, lässt dies jedoch nicht $\mathrm{zu}$.

Die Hauptschwierigkeit besteht jedoch in der Ermittlung von $\nabla^{2} f\left(x_{k}\right)$, bzw. der dafür eingeführten approximierten Hesse-Matrix $B_{k}$. Drei Möglichkeiten zur Berechnung wurden bereits vorgestellt und werden nachfolgend bezüglich ihrer Eignung für den Bereich der Formoptimierung bewertet:

\section{Gradientenverfahren (Kap. 3.3)}

Das Verfahren ist zwar global konvergent, jedoch meist außerordentlich langsam und sollte deshalb lediglich zur Absicherung besserer Methoden (z.B. im Rahmen des GaussNewton-Verfahrens) eingesetzt werden (Bonnans et al., 1997).

\section{Newton-Verfahren (Kap. 3.4)}

Diese Methode kann sehr schnell konvergieren, enthält jedoch die echte Hesse-Matrix. Da hierfür die analytischen Ableitungen der Zielfunktion nach den Parametern notwendig wären, diese jedoch nicht zur Verfügung stehen, kann die Methode nicht genutzt werden.

\section{Gauss-Newton-Verfahren (Kap. 3.5)}

Mathematisch betrachtet entspricht das Trust-Region Gauss-Newton-Verfahren dem Levenberg-Marquardt-Verfahren. Wie zu Beginn des Abschnitts erläutert, eignet es sich nur für die Lösung von FQM-Problemen, welches hier jedoch nicht vorliegt.

Eine weitere Möglichkeit zur Approximation der Hesse-Matrix stellen die ebenfalls auf dem Newton-Schritt basierenden Quasi-Newton-Verfahren dar. 


\subsection{Quasi-Newton-Verfahren}

Um eine gute Abstiegsrichtung zu ermitteln, genügt zunächst eine Näherung der HesseMatrix. Ein entsprechender Ansatz ist die Abschätzung auf Basis der Änderung der ersten Ableitungen von der Entwicklungsstelle $x_{k-1} \mathrm{zu} x_{k}$, welche implizit auch Informationen über die zweiten Ableitungen enthalten. Eine einfache dementsprechende Bildungsvorschrift (Miller, 2000) lautet

$$
\nabla^{2} f\left(x_{k}\right) \approx B_{k}=\frac{\nabla f\left(x_{k}\right)-\nabla f\left(x_{k-1}\right)}{x_{k}-x_{k-1}}
$$

und stellt einen rückwärtigen Differenzenquotienten dar, dessen Störung der Schrittweite des vergangenen Schrittes entspricht. Da meist jedoch auch die ersten Ableitungen nur in Form von Differenzenquotienten zur Verfügung stehen, kann die Genauigkeit gering bzw. die Fehlerfortpflanzung problematisch sein. Liegen die Entwicklungsstellen weit auseinander, was insbesondere im Bereich der Formoptimierung der Fall ist, verstärkt sich dies noch.

Ein weiterer Aspekt der Quasi-Newton-Verfahren ist, dass die Approximation von $B_{k}$ nicht in jedem Schritt komplett neu berechnet, sondern lediglich aufgefrischt wird. Ziel ist es, den Unterschied zwischen $B_{k}$ und $B_{k+1}$ möglichst klein zu halten, die Änderung der Gradienten jedoch angemessen zu berücksichtigen. Dabei sollte berücksichtigt werden, dass die Approximation $B$ folgende Eigenschaft der echten Hesse-Matrix erfüllt:

$$
\nabla^{2} f\left(x_{k+1}\right) \cdot\left(x_{k+1}-x_{k}\right) \approx \nabla f\left(x_{k+1}\right)-\nabla f\left(x_{k}\right) .
$$

Durch Einsetzen von $d_{k}=x_{k+1}-x_{k}$ und $y_{k}=\nabla f\left(x_{k+1}\right)-\nabla f\left(x_{k}\right)$ ergibt sich die auch als Sekantenbedingung bezeichnete Formulierung

$$
B_{k+1} d_{k}=y_{k}
$$

Um eine eindeutige Approximationsmatrix zu erhalten, wird zudem üblicher Weise die Symmetrie von $B_{k+1}$ gefordert sowie bei einigen Zugängen die positive Definitheit.

Unabhängig davon, welches Auffrischungsverfahren genutzt wird, muss zu Beginn der Optimierung eine Startmatrix $B_{0}$ gewählt werden. Während die Einheitsmatrix zwar die einfachste Wahl darstellt, jedoch eine schlechte Leistung des gesamten Algorithmus zur Folge hat, stellt eine an der Größenordnung der Zielfunktion orientierte Startmatrix die bessere Wahl dar (Dennis und Schnabel, 1996), zumal dieser beim Start des Optimierungsalgorithmus zur Verfügung steht:

$$
B_{0}=\left|f\left(x_{0}\right)\right| I \text {. }
$$

In der Praxis kann eine noch bessere Startmatrix generiert werden, wenn im Rahmen der Gradientenberechnung in Form von Differenzenquotienten die Formparameter gestört und die zugehörigen Zielfunktionswerte berechnet werden und somit zur Verfügung stehen. Auf deren Basis kann die Startmatrix gemäß Gl. 4.4 bestimmt werden. Die Berücksichtigung der Skalierung der einzelnen Parameter bei der Berechnung von $B_{0}$ bleibt davon unberührt.

Es existieren verschiedene Zugänge zur Auffrischung der approximierten Hesse-Matrix, von denen einige populäre Vertreter in SPC-Opt integriert sind: 


\section{BFGS-Auffrischung}

Die BFGS-Auffrisch-Formel wurde durch die Namensgeber Broyden (1970), Fletcher (1970), Goldfarb (1970) sowie Shanno (1970) unabhängig voneinander vorgeschlagen und lautet

$$
B_{k+1}=B_{k}-\frac{B_{k} d_{k} d_{k}^{T} B_{k}}{d_{k}^{T} B_{k} d_{k}}+\frac{y_{k} y_{k}^{T}}{y_{k}^{T} d_{k}} .
$$

Für diesen ungedämpften BFGS-Algorithmus, muss die Anstiegsbedingung

$$
d_{k}^{T} y_{k}>0
$$

eingehalten werden. Die Auffrischung erzeugt mit einigen Absicherungen auf Basis einer positiv definiten Startmatrix eine stets symmetrische und positiv definite neue Approximation der Hesse-Matrix.

Für den Fall, dass die Anstiegsbedingung Gl.4.9 nicht erfüllt ist, muss eine entsprechende Absicherung implementiert werden. Dies kann z.B. in Form eines Aussetzens der Auffrischung erfolgen. Besser ist jedoch die Ergänzung eines Dämpfungsterms (Nocedal und Wright, 1999):

$$
B_{k+1}=B_{k}-\frac{B_{k} d_{k} d_{k}^{T} B_{k}}{d_{k}^{T} B_{k} d_{k}}+\frac{y_{k} y_{k}^{T}}{y_{k}^{T} d_{k}}+\frac{r_{k} r_{k}^{T}}{d_{k}^{T} r_{k}}
$$

mit

$$
r_{k}=\theta_{k} y_{k}+\left(1-\theta_{k}\right) B_{k} d_{k}
$$

und

$$
\theta_{k}=\left\{\begin{array}{c}
1 \text { für } d_{k}^{T} y_{k} \geq 0,2 d_{k}^{T} B_{k} d_{k} \\
\frac{0,8 \cdot d_{k}^{T} B_{k} d_{k}}{d_{k}^{T} B_{k} d_{k}-d_{k}^{T} y_{k}} \text { für } d_{k}^{T} y_{k}<0,2 d_{k}^{T} B_{k} d_{k} .
\end{array}\right.
$$

Aufgrund seiner guten Eigenschaften stellt das BFGS-Verfahren in SPC-Opt die Standardmethode zur Approximation der Hesse-Matrix dar.

\section{SR1-Auffrischung}

Die Auffrisch-Formel dieses Verfahrens (symmetric-rank-one) ist wie folgt gegeben (Miller, 2000):

$$
B_{k+1}=B_{k}+\frac{\left(y_{k}-B_{k} d_{k}\right)\left(y_{k}-B_{k} d_{k}\right)^{T}}{\left(y_{k}-B_{k} d_{k}\right)^{T} d_{k}} .
$$

Hierbei ist entsprechend (Nocedal und Wright, 1999) eine Absicherung notwendig. Diese besteht darin, dass keine Auffrischung stattfindet, falls mit $r=10^{-8}$ gilt:

$$
\left|d_{k}^{T}\left(y_{k}-B_{k} d_{k}\right)\right|<r\left\|d_{k}\right\|\left\|y_{k}-B_{k} d_{k}\right\| \text {. }
$$

Der Ansatz hat gegenüber der BFGS-Formel den Vorteil, dass die zweite Ableitung genauer berechnet wird. Damit verbunden ist aber auch die Abbildung eventueller negativer Krümmungen, womit $B_{k+1}$ nicht zwangsläufig positiv definit (wenn auch stets symmetrisch) ist. Falls das verwendete Optimierungsverfahren ein positiv definites 
$B_{k+1}$ erfordert, können verschiedene zur Verfügung stehende Regularisierungsverfahren zur nachträglichen Absicherung der positiven Definitheit verwendet werden.

\section{DFP-Auffrischung}

Der Ansatz zu Auffrischung von $B_{k}$ ergibt sich zu (Nocedal und Wright, 1999):

$$
B_{k+1}=\left(I-\gamma_{k} y_{k} d_{k}^{T}\right) B_{k}\left(I-\gamma_{k} d_{k} y_{k}^{T}\right)+\gamma_{k} y_{k} y_{k}^{T} \text { mit } \gamma_{k}=\left(y_{k}^{T} d_{k}\right)^{-1}
$$

Auch hier ist eine Absicherung notwendig, welche äquivalent zum ungedämpften BFGSVerfahren erfolgt und im Aussetzen der Auffrischung besteht, falls $y_{k}^{T} d_{k}<0$ ist.

\subsection{Bewertung der Trust-Region-Verfahren}

Aufgrund der praktischen Erfahrungen bezüglich des Einsatzes des implementierten TrustRegion-Verfahren lässt sich feststellen, dass die Konvergenzgeschwindigkeit maßgeblich davon abhängt,

- ob das Optimierungsproblem gut oder schlecht gestellt ist,

- wie stark die Designvariablen miteinander korrelieren und

- in welcher Art die Verfahrensparameter gewählt werden.

Insbesondere bei der Wahl der Steuergrößen des TR-Verfahrens muss abgeschätzt werden, ob das sichere Auffinden des Minimums der Zielfunktion wichtiger ist als die unbedingte Reduktion der dafür notwendigen Optimierungsschritte. Dies ist besonders dann schwierig, wenn die Berechnung der einzelnen Funktionswerte sehr zeitaufwändig ist und die Optimierung potentiell mehrere Tage zur Lösung benötigt.

Neben den Trust-Region-Verfahren wurden in SPC-Opt auch verschiedene Line-Search-Strategien integriert. Dies erfolgte auf Grundlage der Arbeit von Clausner (2007), in welcher die verschiedenen Verfahren zusammengetragen und hinsichtlich ihrer Eigenschaften untersucht wurden. Zwar ist die grundsätzliche Funktionsweise der beiden Verfahren gleich, jedoch unterschieden sie sich in der Art und Weise, wie von $x_{k}$ zu $x_{k+1}$ gegangen wird. Bei den Trust-Region-Verfahren wird zuerst eine maximale Schrittweite festgelegt und danach die Schrittrichtung mit den besten Ergebnissen gewählt. Für Line-Search-Strategien hingegen stellt die Ermittlung des Schrittvektor die Grundvoraussetzung dar und erst danach wird die Schrittweite festgelegt. Wie die Schrittrichtung bestimmt wird (Gradientenverfahren, newtonbasierte Schritte, Trust-Region-Schritte), ist für einen Line-Search-Algorithmus unerheblich. Es muss lediglich sichergestellt sein, dass es sich beim Schrittvektor $d_{k}$ um eine Abstiegsrichtung handelt.

Die Berechnung der neuen Entwicklungsstelle erfolgt nunmehr nach:

$$
x_{k+1}=x_{k}+\alpha d_{k}
$$

Hierbei stellt $\alpha$ einen Faktor dar, der die Schrittweite in Richtung $d_{k}$ verändert, bis eine akzeptable Schrittweite erreicht ist. Es wird nun eine Funktion $\Phi(\alpha)$ erstellt, welche die 
Abhängigkeit der Funktionswerte von $f$ entlang des Schrittvektors $d_{k}$ mit $\alpha$ als Schrittweite darstellt. Die Herausforderung der Line-Search-Strategie besteht in der Bestimmung eines $\alpha>0$, sodass ein Abstieg in $\Phi$ und somit auch in $f$ erreicht wird. Hier tritt jedoch das Problem auf, dass $\Phi$ praktisch nie in analytischer Form vorliegt. Die einzige Möglichkeit besteht demnach in der Durchführung von Testrechnungen zur näherungsweisen Abbildung dieser Funktion.

Die Testrechnungen müssen für verschiedene $\alpha_{j}$ charakterisiert werden, wofür sie in drei Kategorien eingeteilt werden - akzeptabel, zu groß oder zu klein. Hierfür existieren verschiedene Akzeptanzkriterien, z.B. das Armijo-Kriterium, die Goldstein-Kriterien und die Wolfe-Kriterien (Alt, 2011). Darüber hinaus wird ein Auffrischverfahren benötigt, welches im Falle einer nicht akzeptablen Schrittweite $\alpha_{j}$ vorgibt, auf welche Weise ein vermutlich besseres $\alpha_{j+1}$ bestimmt wird. Beispiele dafür sind Verfahren mit konstanten Faktoren bzw. Summanden, sowie Verfahren mit quadratischen oder kubischen Polynomen. Aufgrund der weit verbreiteten Nutzung von Line-Search-Verfahren existiert eine Reihe guter Übersichten zur Funktionsweise, u.a. (Nocedal und Wright, 1999; Bhatti, 2000; Alt, 2011).

Beim Vergleich der Line-Search-Strategien mit den Trust-Region-Verfahren besitzen erstere den Vorteil, dass deutlich bessere und detailliertere Abbruchkriterien bei der Suche nach $\alpha$ existieren, da diese Mischungen aus Abstiegs- und Krümmungskriterien sein können. Zudem sind Line-Search-Verfahren skalierungsunempfindlicher. Bei einer guten Wahl von $\alpha$ konvergieren sie deutlich schneller als die Trust-Region-Verfahren. Ein großer Schwachpunkt ist jedoch die Abhängigkeit von der Schrittrichtung im ersten Optimierungsschritt. Ist das Ersatzmodell an der Entwicklungsstelle $x_{0}$ nicht gut, bricht die Optimierung ab, da die Schrittweite des ersten Probeschrittes gewöhnlich dem natürlichen Newton- oder Gradientenschritt entspricht. Zudem sind die optimalen Steuerparameter der Line-Search-Verfahren von der Problemstellung abhängig, was sich auch darin äußert, dass dementsprechende Empfehlungen je nach Autor variieren. Dies ist nicht nur für praxisorientierte Anwender ohne umfangreiche Kenntnisse dieser Thematik problematisch, sondern auch für versierte Nutzer, welche Aufgaben der Formoptimierung ohne Kenntnis der Zielfunktionstopologie oder einem guten Startparametersatz bearbeiten. Aus diesem Grund enthält die vorliegende Arbeit keine detaillierte Betrachtung der Line-Search-Verfahren bzw. entsprechende Anwendungsbeispiele. Die Nutzung des Trust-Region-Verfahrens zur Lösung eines Formoptimierungsproblems hingegen wird anhand des nachfolgenden Beispiels demonstriert.

\subsection{Beispiel Lochscheibe}

Für das Beispiel einer biaxial belasteten Scheibe mit einem Loch entsprechend Bild 4.2 im Ausgangszustand soll die Randkontur $\Omega$ derart verändert werden, dass die größte Hauptspannung $\sigma_{1}$ für eine gegebene Belastung am Rand $\Omega$ und eine vorgegebene Lochweite in Richtung der beiden Koordinatenachsen minimal wird. Es handelt sich dabei um einen gleichmäßigen zweiachsigen Zug bei Annahme des ebenen Verzerrungszustandes (EVZ). Das Material verhält sich elastisch-plastisch mit isotroper Verfestigung nach Ulbricht und Röhle (1975). 


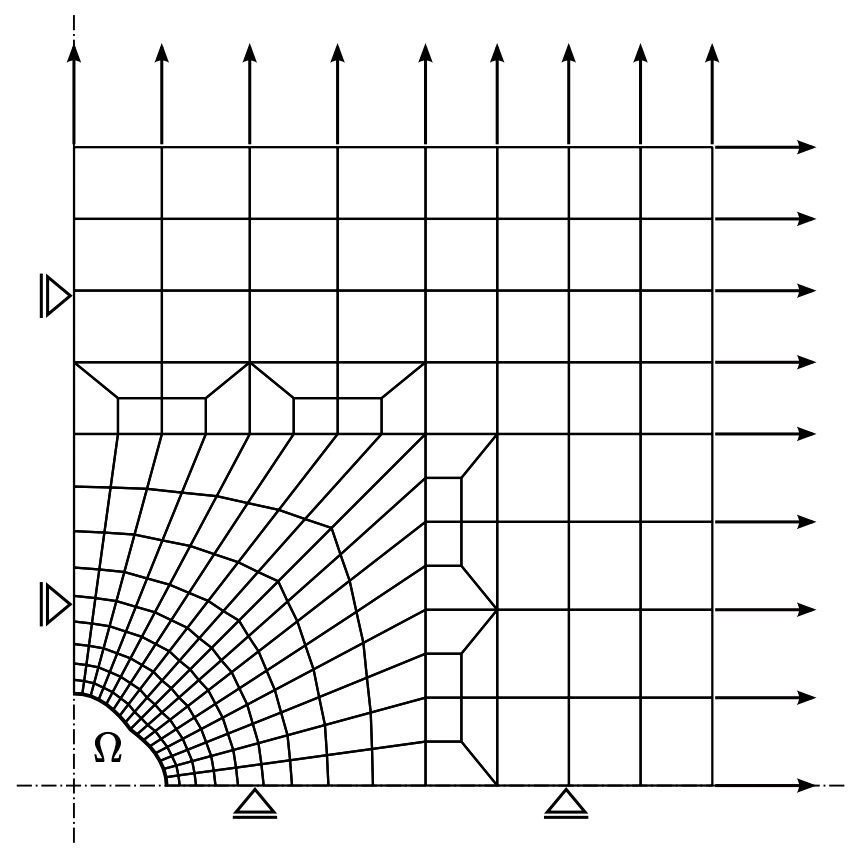

Bild 4.2: Scheibe mit Loch (ein Viertel), Vernetzung und Randbedingungen.

Mit den Designvariablen $\underline{p}$ wird die Form der durch NURBS approximierten Randkontur $\Omega$ festgelegt. Die Lochweite in Richtung der beiden Koordinatenachsen $x$ und $y$ ist identisch und bleibt während der Identifikation konstant. Die entsprechende Zielfunktion $\Phi$ lautet

$$
\Phi(\underline{p})=\sigma_{1}(\underline{X}, \underline{p}) \rightarrow \min _{p}
$$

Da sich die Zielfunktion nicht auf ein FQM-Problem zurückführen lässt, erfolgt die Optimierung durch Nutzung des in Kap. 4.1 beschriebenen Trust-Region-Verfahrens. Die Approximation der Hesse-Matrix erfolgt mit dem implementierten BFGS-Verfahren. Bei Verwendung von 4 Designvariablen endet die Formoptimierung nach 8 Optimierungsschritten und führt auf die in Bild 4.3 dargestellte Kontur des Loches. Es sei darauf hingewiesen, dass es sich bei der optimierten Kontur nicht um einen Kreisabschnitt handelt.
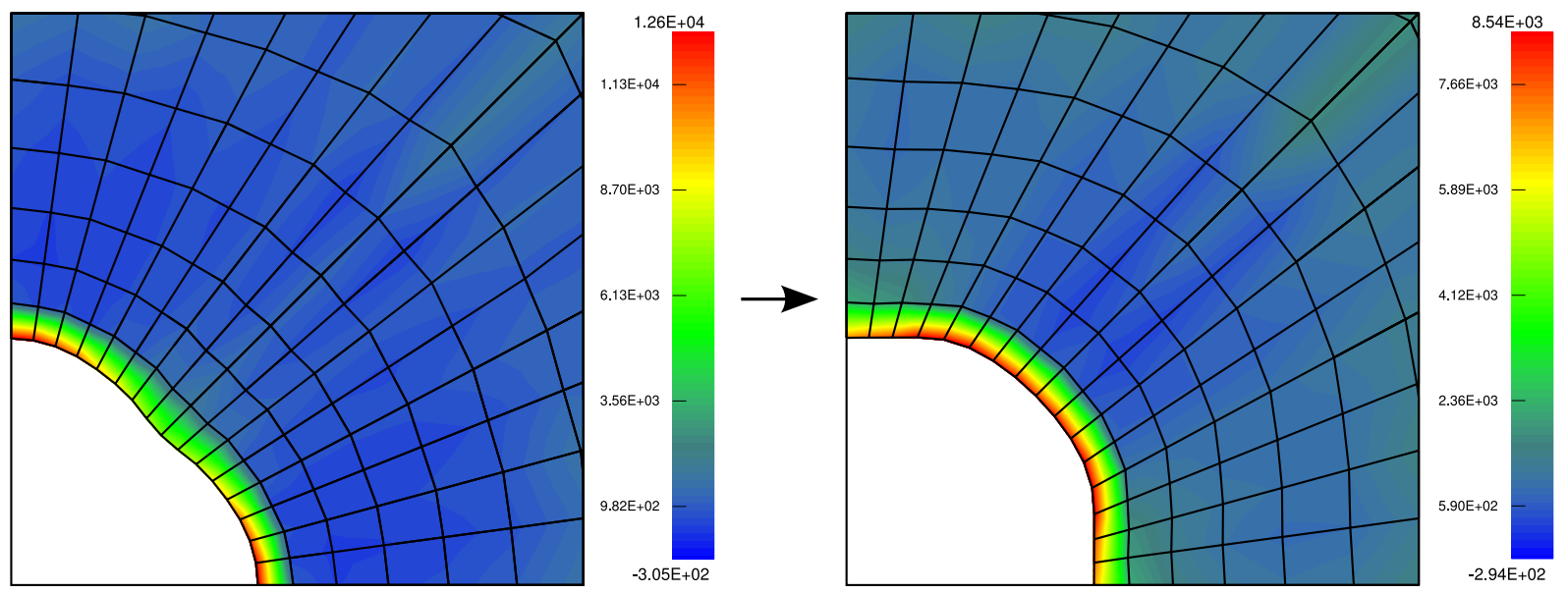

Bild 4.3: Verteilung der Hauptspannung $\sigma_{1}$ für den Startvektor $\underline{p}_{0}$ und den optimalen Vektor $\underline{p}_{\text {end }}$, dargestellt im Netz des undeformierten Zustands. 
Um das Ergebnis quantitativ bewerten zu können, sind die ersten Hauptspannungen der Knoten nach der Simulation auf der Lochkontur in Tabelle 4.1 aufgeführt.

Tabelle 4.1: Hauptspannung $\sigma_{1}$ in den Knoten entlang der Randkontur $\Omega$.

\begin{tabular}{|c|c|c|}
\hline Knotennummer & $\sigma_{1}[\mathrm{MPa}]$ für $\underline{p}=\underline{p}_{0}$ & $\sigma_{1}[\mathrm{MPa}]$ für $\underline{p}=\underline{p}_{\text {end }}$ \\
\hline 362 & 1256 & 696 \\
257 & 1245 & 738 \\
213 & 1154 & 769 \\
246 & 1096 & 853 \\
210 & 961 & 845 \\
235 & 896 & 854 \\
211 & 786 & 812 \\
219 & 743 & 818 \\
212 & 686 & 793 \\
296 & 742 & 818 \\
290 & 785 & 812 \\
308 & 895 & 853 \\
302 & 960 & 845 \\
320 & 1096 & 853 \\
314 & 1154 & 769 \\
332 & 1245 & 738 \\
326 & 1256 & 696 \\
\hline
\end{tabular}

Es ist deutlich sichtbar, dass die ermittelten Designvariablen $\underline{p}=\underline{p}_{\text {end }}$ zu einer deutlichen Verringerung des größten Wertes der Hauptspannung $\sigma_{1}$ auf der Randkontur $\Omega$ führen. Da in jedem Optimierungsschritt der Maximalwert von $\sigma_{1}$ in einem anderen Knoten des Randes $\Omega$ auftreten kann, wird dadurch das Konvergenzverhalten negativ beeinflusst. 


\section{Startparametervariation}

Die bislang vorgestellten und in SPC-Opt integrierten Methoden zur Parameteridentifikation und Formoptimierung gehören zu den gradientenbasierten Verfahren, welche gewöhnlich in das nächstgelegene Minimum führen. In den meisten Fällen ist allerdings das globale Minimum von Interesse, dessen Bestimmung beim Vorhandensein mehrerer unterschiedlicher lokaler Minima jedoch nicht ohne Weiteres möglich ist. Eine übliche Vorgehensweise besteht daher in der Durchführung hinreichend vieler Optimierungen mit unterschiedlichen Startvektoren, von welchen das lokale Minimum mit dem kleinsten Zielfunktionswert als globales Minimum angenommen wird. Beansprucht bereits eine einzelne Optimierung erhebliche Zeit, was bei praktischen Problemstellungen häufig der Fall ist, muss die Zahl der Startvektoren zwangsläufig begrenzt werden. Das naheliegende Interesse gilt daher der optimalen Auswahl von Startvektoren, um so ein Maximum an Informationen über die Topologie der Zielfunktion zu erhalten. Hierfür eignen sich Ansätze aus dem Bereich der Versuchsplanung, auch als Design of Experiments (DOE) bezeichnet (Giunta et al., 2003). Die Grundlage der Implementierung eines Moduls zur Startparametervariation in SPC-Opt stellt die Arbeit von Landgraf (2008) dar, in welcher die Vor- und Nachteile verfügbarer Ansätze zu dieser Thematik untersucht und bewertet wurden.

\subsection{Methoden zur Erzeugung von Startvektoren}

Zur Einordnung der globalen Optimierungsverfahren entsprechend ihrer Eigenschaften existieren unterschiedliche Ansätze, bezüglich derer Törn und Žilinskas (1989) einen guten Überblick darstellen. Für die Implementierung in $S P C$ - $O p t$ sind aus programmiertechnischer Sicht diejenigen Methoden interessant, welche gut in die modulare Programmstruktur passen und der eigentlichen Optimierung vorgeschaltet werden können. Zudem müssen infolge der Verwendung gradientenbasierter Verfahren bei den meisten Aufgabenstellungen nur wenige Parameter identifiziert bzw. optimiert werden. Die Auswertung der Zielfunktion (meist in Form einer FEM-Simulation) ist jedoch potentiell sehr rechenintensiv. Im Hinblick darauf eignen sich Verfahren, welche entsprechend der vorgegebenen Zahl Startvektoren ein möglichst gutes Raum füllendes Design erzeugen (Schumacher, 2005). Dies erfolgt zunächst für einen Entwurfsraum $\mathcal{X}=[0,1]^{d}$, wobei $d$ der Anzahl der Parameter entspricht. Benötigt wird weiterhin die Definition der Unter- und Obergrenzen $p_{i}^{(l)}$ bzw. die $p_{i}^{(u)}$ eines jeden Parameters, welche durch den Anwender festgelegt werden müssen und meist auf Erfahrungswerten, konstruktiven Beschränkungen (Formparameter) oder physikalischen sinnvollen Grenzen (z.B. Materialparameter) basieren. Dadurch ergibt sich der zulässige Parameterraum $\mathcal{P}$ :

$$
\mathcal{P}=\left(\begin{array}{c}
p_{1}^{(l)} \leq p_{1} \leq p_{1}^{(u)} \\
p_{2}^{(l)} \leq p_{2} \leq p_{2}^{(u)} \\
\cdots \\
p_{d}^{(l)} \leq p_{d} \leq p_{d}^{(u)}
\end{array}\right)
$$


Die Rücktransformation aus dem Entwurfsraum in den Parameterraum und die damit verbundene Skalierung erfolgt entsprechend

$$
p_{j}^{i}=\left(p_{j}^{(u)}-p_{j}^{(l)}\right) x_{j}^{i}+p_{j}^{(l)},
$$

wobei $\left(p_{j}^{(u)}-p_{j}^{(l)}\right)$ die Skalierung der jeweiligen Achse $j$ und $x_{j}^{i}$ die Koordinate im Entwurfsraum darstellt. Die durch $x_{j}^{i}$ beschriebenen Vektoren im Entwurfsraum werden auch als "Designpunkte" bezeichnet. Mit Hilfe der sogenannten Sampling-Methoden werden die $m$ Designpunkte ermittelt und deren Koordinaten in der Designmatrix $X^{D}$ gespeichert werden. Dies erfolgt in der Form $X_{i}=\left\{X_{i 1}, X_{i 1}, \ldots, X_{i d}\right\}$, wobei $i=1,2, \ldots, m$ die Designpunkte darstellen.

\section{Gittermethoden}

Bei diesem deterministischen Ansatz wird der Parameterraum $\mathcal{X}$ mit einem Gitter versehen, an welchem die Designpunkte ausgerichtet werden (hier: Ausrichtung an den Mittelpunkten der Zellen des Gitters). Im einfachsten Fall verlaufen die Gitterstränge parallel zu den Achsen des Versuchsraumes (Grossmann, 1997) und werden jeweils in $k$ äquidistante Intervalle aufgeteilt. Ein für $d$ Dimensionen erzeugtes Design enthält demnach $m=k^{d}$ Designpunkte. Diese Methode hat den Nachteil, dass die Anzahl der Startvektoren nicht frei wählbar ist und außerordentlich groß sein kann. Zudem wird für die verschiedenen Designpunkte jeder Parameterwert mehrfach verwendet, was im Hinblick auf das weitestgehend deterministische Verhalten einer Simulation nur wenige neue Informationen liefert und daher für Computerexperimente als ungeeignet angesehen wird (Koehler und Owen, 1996). Eine Modifikation dieses Verfahrens stellt die Methode der Good-Latice-Points (GLP) dar. Gittermethoden sind aufgrund der vorgegebenen Anzahl Designpunkte nicht in SPC-Opt implementiert, finden jedoch Eingang in die nachfolgenden Methoden.

\section{Random Sampling}

Zu den stochastischen Verfahren, welche die Designpunkte zufällig auswählen, gehört die einfache Methode des Random Sampling, auch Monte Carlo Sampling genannt (Giunta et al., 2003). Hierbei werden die Random Designs (RD) zufällig einer gleichverteilten Zahlenmenge $U[0,1]$ entnommen. Der Nachteil der Methode besteht darin, dass die Verteilung der Startvektoren im Entwurfsraum sehr schlecht sein kann. Durch (Burkardt, 2008) wird eine Modifikation des Verfahrens vorgeschlagen, bei welcher die zufällige Auswahl auf diskrete Punkte im Entwurfsraum beschränkt wird. Diese werden auf zuvor entsprechend der Vorgehensweise beim Gitterverfahren generiert.

\section{Latin Hypercube Sampling}

Das von McKav et al. (1979) vorgestellte Verfahren stellt eine Verbesserung gegenüber dem Random Sampling dar. Analog zu den Gitterverfahren wird für die Erzeugung eines Latin Hypercube Design (LHD) zunächst ein Gitter im Entwurfsraum $\mathcal{X}$ erzeugt. Abweichend davon ist die Aufteilung $k$ der Gitterstränge jedoch nicht frei, sondern entspricht der Anzahl der zu erzeugenden Designpunkte $m$. Die Auswahl der Zellen 
erfolgt zufällig und unter der Bedingung, dass deren Projektion auf die Koordinatenachsen eine Gleichverteilung ergibt. Dabei kann die Positionierung der Designpunkte, ausgedrückt durch die $m \times d$ Matrix $U$, innerhalb einer Zelle zufällig oder fest definiert erfolgen. In $S P C$-Opt werden dafür die Zellenmittelpunkte genutzt, wodurch sich für alle Designpunkte $U_{i j}=0,5$ ergibt. Die Berechnungsvorschrift der Designmatrix $X^{D}$ lautet:

$$
X_{i j}^{D}=\frac{\Pi_{i j}-U_{i j}}{m} \quad \text { mit } \quad i=1,2 \ldots, m ; j=1,2, \ldots, d,
$$

wobei $\Pi$ eine Permutationsmatrix darstellt (Park, 1994), deren Spalten Permutationen von $\{1, \ldots m\}$ enthalten.

Im Vergleich zu den RDs sind die Designpunkte von LHDs deutlich besser verteilt. Allerdings stellt nicht jedes LHD zwangsläufig eine gute Raum füllende Verteilung dar. Dies wird durch das zweidimensionale Beispiel in Bild 5.1 veranschaulicht, welches zwei gültige LHDs zeigt, von denen letzteres den Raum nur sehr schlecht ausfüllt.

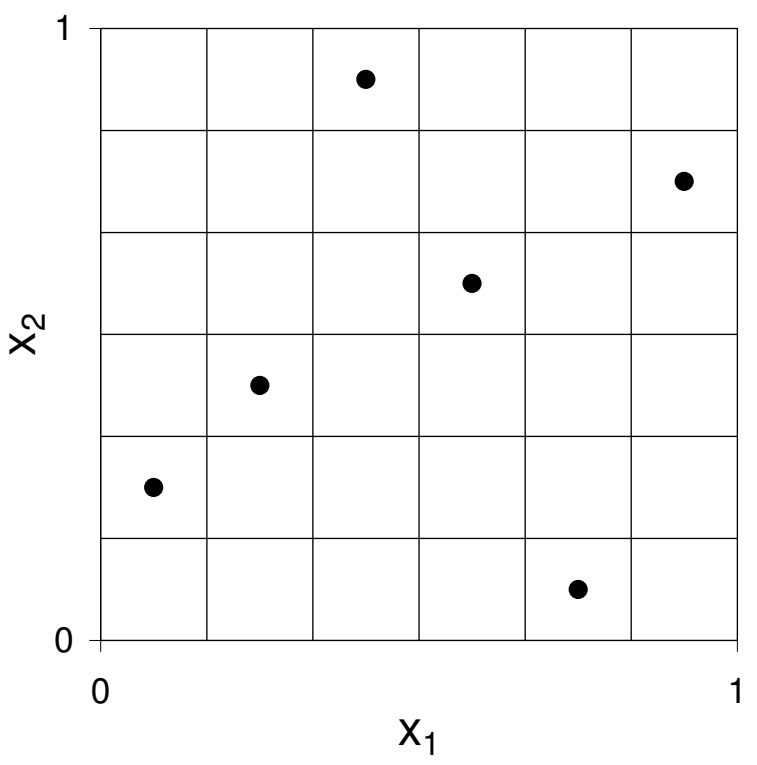

(a) LHD mit guter Verteilung

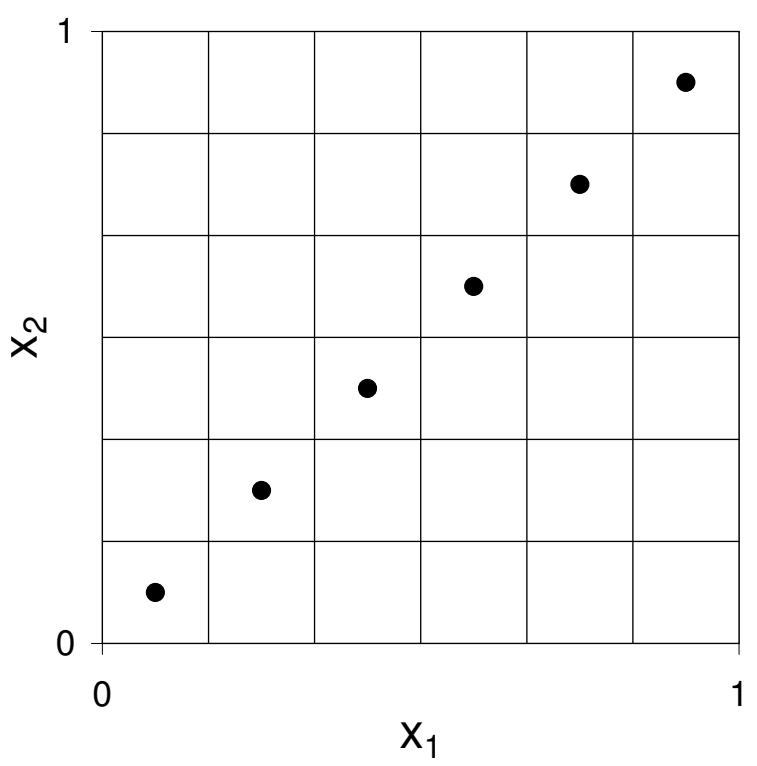

(b) LHD mit schlechter Verteilung

Bild 5.1: Latin Hypercube Designs für $d=2$ und $m=6{ }^{*}$

Für die Verbesserung der Eigenschaften von LHDs können verschiedene Vorgehensweisen genutzt werden (Grary, 2002). Eine gute Möglichkeit stellt das Symmetrische Latin Hypercube Design (SLHD) dar (Ye et al., 2000), welches eine beliebige Anzahl Designpunkte erzeugen kann, jedoch bessere Eigenschaften gegenüber den LHD aufweist. Die Berechnung der SLHD erfolgt analog zum LHD entsprechend G1. 5.3, jedoch unter der Bedingung, dass für eine Zeile $\left(a_{1}, a_{2}, \ldots a_{d}\right)$ der Permutationsmatrix eines SLHD eine andere Zeile der Form $\left(m+1-a_{1}, n+1-a_{2}, \ldots n+1-a_{d}\right)$ ebenfalls Teil dieser Matrix ist. Grafisch ist dies für eine gerade Anzahl Designpunkte in Bild 5.2 dargestellt und zeigt, dass die Einhaltung der Bedingung einer Punktspiegelung am Zentrum von $\mathcal{X}$ entspricht.

*Darstellung entstammt (Landgraf, 2008$)$ 
Falls eine ungerade Anzahl Designpunkte vorliegt, wird eine weitere Bedingung benötigt, da genau ein Punkt keinen zugehörigen Punkt entsprechend obiger Bedingung besitzt. Zur Beibehaltung der Symmetrie wird deshalb per Definition ein Punkt exakt im Zentrum des Entwurfsraumes gesetzt. Für die übrigen Designpunkte gilt die oben genannte Bedingung und führt z.B. auf das in Bild 5.3 dargestellte Design.

$$
\Pi_{\text {even }}=\left(\begin{array}{ll}
1 & 2 \\
2 & 4 \\
3 & 6 \\
4 & 1 \\
5 & 3 \\
6 & 5
\end{array}\right)
$$

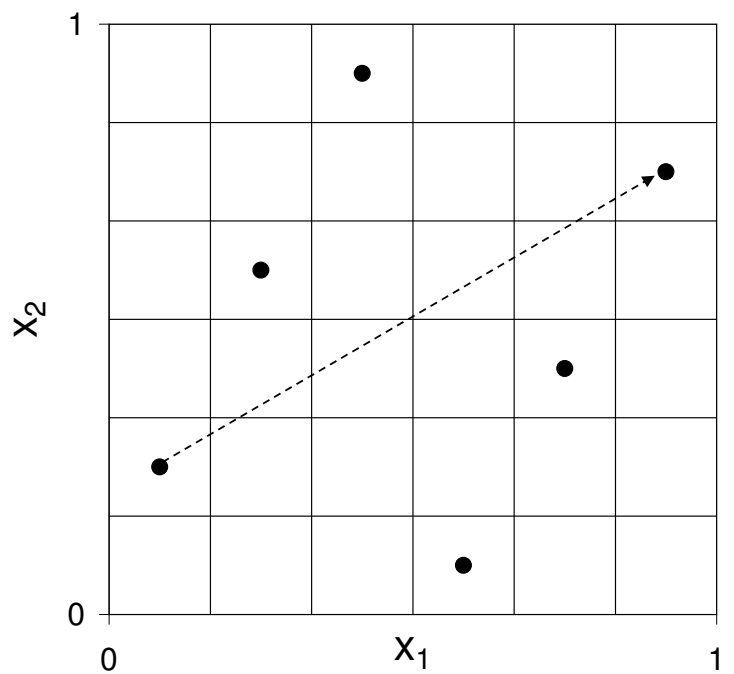

Bild 5.2: Symmetrisches Latin Hypercube Design für $d=2$ und $m=6$.*

$$
\Pi_{\text {odd }}=\left(\begin{array}{cc}
1 & 4 \\
2 & 1 \\
3 & 3 \\
4 & 5 \\
5 & 2
\end{array}\right)
$$

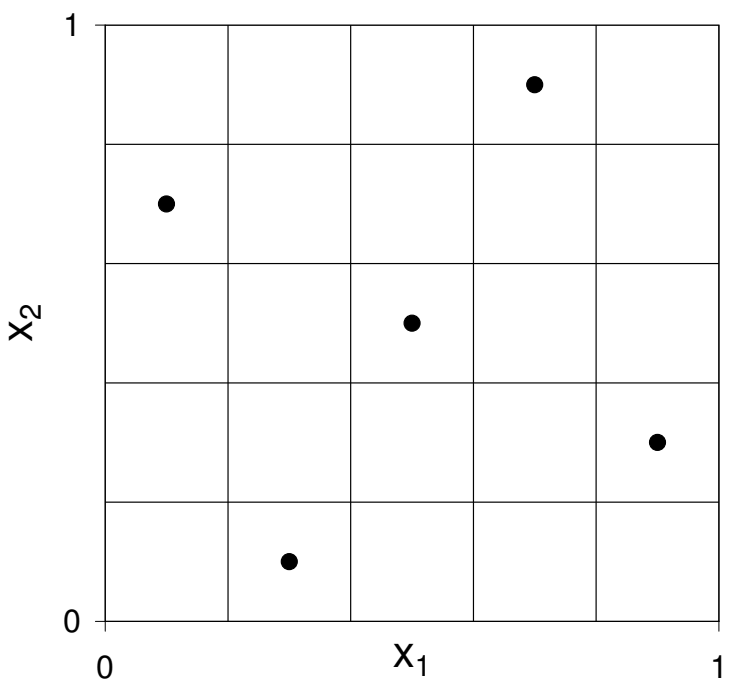

Bild 5.3: Symmetrisches Latin Hypercube Design für $d=2$ und $m=5 . *$

\subsection{Optimierung der Startdesigns}

Bezüglich der Raumfüllung sind die mit den in Kap. 5.1 vorgestellten Sampling-Verfahren erzeugten RDs, LHDs sowie SLHDs nicht zwangsläufig gut. Es erfolgt deshalb eine Optimierung der Startdesign anhand geeigneter Designkriterien, welche nachfolgend vorgestellt werden. Mathematisch ausgedrückt wird das Design $D^{*} \in \mathcal{D}$ mit $\mathcal{D} \subset \mathbb{R}^{d}$ gesucht, für welches eine auf Basis der Designkriterien formulierte Zielfunktion $\phi(D)$ mit $D \in \mathcal{D}$ minimal wird:

$$
\phi\left(D^{*}\right)=\min _{D \in \mathcal{D}} \phi(D) .
$$

*Darstellung entstammt (Landgraf, 2008) 


\subsubsection{Designkriterien}

Die zur Beurteilung der Güte eines Designs verwendeten Größen, im Weiteren Designkriterien genannt, können in drei verschiedene Klassen unterteilt werden. Diese werden nachfolgend in kompakter Form vorgestellt. Für detaillierte Ausführungen sei auf die jeweilig angegebenen Quellen verwiesen.

\section{Designkriterien basierend auf Abstandsmessungen}

Designkriterien dieser Kategorie basieren auf der Messung von Abständen im $d$-dimensionalen euklidischen Raum $\mathbb{R}^{d}$. Hierfür wird für die Berechnung des Abstandes $\delta_{p}$ zweier Punkte $x^{1}, x^{2} \in \mathbb{R}^{d}$ die Metrik

$$
\delta_{p}\left(x^{1}, x^{2}\right)=\left[\sum_{j=1}^{d}\left|x_{j}^{1}-x_{j}^{2}\right|^{p}\right]^{1 / p}, \quad p \geq 1, \quad p \in \mathbb{N}
$$

eingeführt. Typische Werte von $p$ sind $p=1$ für die L1-Metrik, $p=2$ für die euklidische Metrik und $p=\infty$ für die Maximum-Metrik (Bronstein et al., 2005).

Das weit verbreitete, von Johnson et al. (1990) vorgestellte Maximin-Distance-Designkriterium $\phi_{M m}(D)$ basiert auf der Annahme, dass die optimale Punkteverteilung $D^{*}$ vorliegt, wenn der Abstand der beiden am nächsten zueinander liegenden Punkte maximal ist. Benötigt wird demnach der minimale Wert aller ermittelten Abstände eines Designs, welcher gleichzeitig das Designkriterium $\phi_{M m}(D)$ darstellt:

$$
\phi_{M m}(D)=\min _{x^{(i)}, x^{(j)} \in D} \delta_{p}\left(x^{(i)}, x^{(j)}\right) ; \quad i=1, \ldots, n-1 ; \quad j=i+1, \ldots, m
$$

Abweichend von Gl. 5.4 wird auf Basis von $\phi_{M m}(D)$ kein Minimierungsproblem, sondern ein Maximierungsproblem der Form

$$
\phi_{M m}\left(D^{*}\right)=\max _{D \in \mathcal{D}} \phi_{M m}(D)
$$

gelöst.

Ein Vertreter von Abstandskriterien, welche alle Abstände zwischen Designpunkten bei der Bildung eines skalaren Größe einbeziehen, ist das $\phi_{p}$-Kriterium (Morris und Mitchell, 1995). Als Grundlage dient das Maximin-Distance-Designkriterium, jedoch werden alle Abstände und deren Häufigkeiten des Auftretens in die Bildungsvorschrift einbezogen. Dies erfolgt derart, dass in einem Vektor $J=\left[J_{1}, J_{2}, \ldots J_{b}\right]^{T}$ die Anzahl $b$ des Auftretens aller Abstände zwischen den $m$ Punkten eines Designs $D$ zusammengefasst werden. Die Berechnung des Designkriteriums erfolgt nunmehr nach

$$
\phi_{\phi_{p}}(D)=\left[\sum_{i=1}^{m} J_{i} \delta_{i}^{-p}\right]^{1 / p}, \quad p \geq 1, \quad p \in \mathbb{N},
$$

wobei sich für $p \rightarrow \infty$ das $\phi_{p}$-Kriterium zum Maximin-Distance-Designkriterium ergibt. 
Das Grundprinzip des Audze-Eglais-Designkriterium $\phi_{A F}(D)$ beruht auf dem Prinzip der gegenseitigen Abstoßung physikalischer Teilchen (Ausze und Eglais, 1977; Husslage, 2006). Deren Anordnung in einem System erfolgt naturgemäß derart, dass die potentielle Energie $U$ minimal wird. Dieses Phänomen wird auf die Designpunkte übertragen und $\phi_{A E}(D)$, welches das Äquivalent der potentiellen Energie darstellt, ergibt sich zu

$$
\phi_{A E}(D)=U=\sum_{i=1}^{m-1} \sum_{j=i+1}^{m} \frac{1}{\delta_{2}^{2}\left(x^{(i)}, x^{(j)}\right)}, \quad x^{(i)}, x^{(j)} \in D .
$$

Dabei stellt $\delta_{2}^{2}\left(x^{(i)}, x^{(j)}\right)$ entsprechend Gl. 5.5 das Quadrat der euklidischen Metrik dar.

Von Santer et al. (2003) wurde das Average-Distance-Designkriterium vorgestellt. Auch mit diesem werden alle Abstände eines Designs berücksichtigt. Die Besonderheit besteht hier in der Normierung der Abstände $\delta_{p}\left(x_{i}, x_{j}\right)$ auf einen maximal möglichen Abstand. Dafür wird der Ausdruck $\rho^{1 / p}$ eingeführt, welcher den Abstand zwischen den Eckpunkten von $\mathcal{X}$ darstellt und es ermöglicht, die Abstände von Punkten auch über verschiedenen Dimensionen miteinander zu vergleichen. Die Berechnungsvorschrift von $\phi_{a v}(D)$ lautet

$$
\phi_{a v}(D)=m_{(p, \lambda)}(D)=\left(\frac{1}{\left(\begin{array}{c}
m \\
2
\end{array}\right)} \sum_{x^{(i)}, x^{(j)} \in D}\left[\frac{\rho^{1 / p}}{\delta_{p}\left(x_{i}, x_{j}\right)}\right]^{\lambda}\right)^{1 / \lambda}, \lambda \geq 1 .
$$

\section{Designkriterien zur Beurteilung der Gleichverteilung}

Der Grundgedanke bei diesen Ansätzen besteht darin, den Unterschied zwischen der Punktverteilung eines betrachteten Designs $D$ zu einer absoluten Gleichverteilung in einem Kriterium zu erfassen. Die Messgrößen zur Bestimmung dieser Abweichung werden als $L_{p^{-}}$ Diskrepanzen bezeichnet. Ein auf diese Weise optimiertes Design wird auch als Uniform Design bezeichnet. Ein Überblick zu diesen Kriterien findet sich unter anderem in (Fang et al., 2006) und (Hickernell, 1998). Nachfolgend wird das Grundprinzip anhand der so genannten Stern $L_{p}$-Diskrepanz erläutert.

Den Ausgangspunkt der Betrachtung stellt analog zu den Abstandskriterien ein erzeugtes Design $D$ im Entwurfsraum $\mathcal{X}$ dar. Um die Diskrepanz eines Punktes $x \in D$ zu bestimmen, wird zunächst ein Teil des Entwurfsraumes als Teilraum $\mathcal{U}$ mit dem Hypervolumen $\operatorname{Vol}(\mathcal{U})$ definiert. Für die Stern $L_{p}$-Diskrepanz entspricht dieser Raum der Aufspannung zwischen dem Koordinatenursprung von $\mathcal{X}$ und dem Punkt $x$, also $\mathcal{U}=[0, x]$. Alle Designpunkte von $D$ können nun innerhalb oder außerhalb von $\mathcal{U}$ liegen, wobei $N(D,[0, x])$ die Punkte innerhalb des Teilraumes bezeichnet. Bei einer absoluten Gleichverteilung aller Designpunkte würde der Quotient $N(D,[0, x]) / m$ dem Volumen des zugehörigen Teilraumes entsprechen. In allen übrigen Fällen ist die Differenz zwischen den beiden Werten größer als 0 und wird auch als Diskrepanz am Punkt $x$ bezeichnet:

$$
D_{l}(D, x)=\left|\frac{N(D,[0, x])}{m}-\operatorname{Vol}([0, x])\right|
$$


Die Mittelung aller Diskrepanzen der Punkte eines Designs mit Hilfe der sogenannten $L_{p^{-}}$ Norm führt auf die $L_{p}$-Diskrepanz $D_{p}(D)$ eines Designs $D$. Im Fall der Stern $L_{p}$-Diskrepanz lautet diese:

$$
D_{p}(D)=\left\{\int_{\mathcal{X}}\left|\frac{N(D,[0, x)))}{m}-\operatorname{Vol}([0, x))\right|^{p}\right\}^{1 / p} \quad \text { mit } p \geq 1
$$

Sehr üblich ist die Wahl von $p=2$ (Fang et al., 2003), welche als Stern $L_{2}$-Diskrepanz $D_{2}(D)$ bezeichnet wird und deren Quadrat das Designkriterium $\phi_{D_{2}}(D)$ darstellt. In ausgeschriebener Form und durch Einsetzen in Gl. 5.4 erhält man:

$$
\begin{aligned}
\left(D_{2}(D)\right)^{2} & =\left(\frac{1}{3}\right)^{d}-\frac{2^{1-d}}{m} \sum_{i=1}^{m} \prod_{k=1}^{d}\left(1-x_{i k}^{2}\right)+\frac{1}{m^{2}} \sum_{i=1}^{m} \sum_{j=1}^{m} \prod_{k=1}^{d}\left[1-\max \left(x_{i k}, x_{j k}\right)\right] \\
& =\phi_{D_{2}}(D) .
\end{aligned}
$$

Da die beispielhaft in Bild 5.5 dargestellte $L_{2}$-Diskrepanz nicht invariant gegenüber einer Koordinatenrotation ist, wird sie als eher ungeeignet zur Findung von Uniform-Designs erachtet (Fang et al., 2000). Aus diesem Grund existieren Modifikationen der $L_{2}$-Diskrepanz, von denen die nachfolgenden in SPC-Opt zur Verfügung stehen und sich gegenüber der $L_{2^{-}}$ Diskrepanz hinsichtlich der Definition des Teilraumes $\mathcal{U}$ unterscheiden:

- zentrierte $L_{2}$-Diskrepanz $C D_{2}(D)$ (Aufspannung von $\mathcal{U}$ zwischen $x$ und nächstgelegenem Eckpunkt von $\mathcal{X}$ )

- symmetrische $L_{2}$-Diskrepanz $S D_{2}(D)$ (Aufspannung von zwei Teilräumen $\mathcal{U}_{1}=[0, x]$ und $\mathcal{U}_{2}=[x, 1]$ Kombination zu $\left.\mathcal{U}=\mathcal{U}_{1} \cap \mathcal{U}_{2}\right)$

- wrap-around $L_{2}$-Diskrepanz $W D_{2}$ (Es erfolgt zunächst eine Aufspannung zwischen zwei Punkten $x_{1}$ und $x_{2}$. Der Berechnung von $W D_{2}$ liegt jedoch nicht dieser Raum, sondern die Vereinigung derjenigen Räume zugrunde, die durch Projektion von $\mathcal{U}=\left[x^{(1)}, x^{(2)}\right]$ auf die Ränder von $\mathcal{X}$ entstehen (vgl. Bild 5.5)

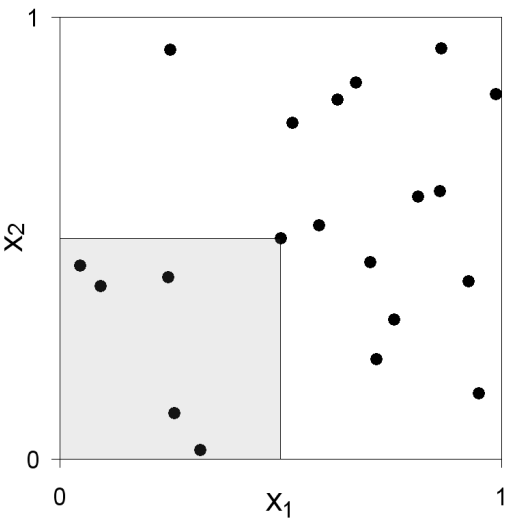

Bild 5.4: Stern $L_{2}$-Diskrepanz.*

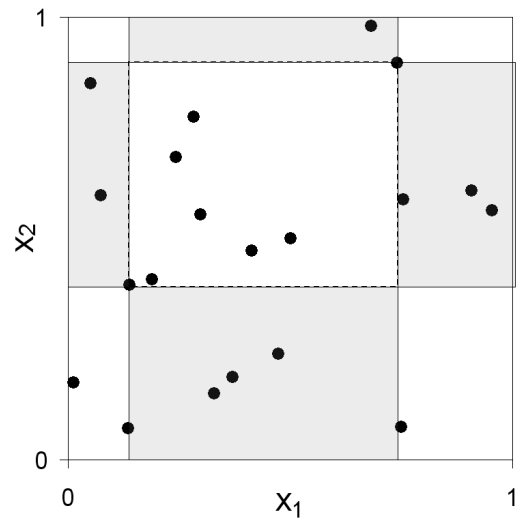

Bild 5.5: Wrap-around $L_{2}$-Diskrepanz.*

${ }^{*}$ Darstellung entstammt (Landgraf, 2008) 
Durch die Modifikation sind die Werte dieser $L_{2}$-Diskrepanzen unabhängig von der Reihenfolge der Punkte in der Designmatrix, invariant gegenüber Koordinatenrotationen und sie betrachten auch die Verteilung von Punkten in nieder-dimensionalen Räumen. Für nähere Ausführungen sei auf Hickernell (1998) verwiesen.

\section{Designkriterien zur Beurteilung statistischer Eigenschaften}

Die abschließend vorgestellte Klasse von Designkriterien basiert auf der Ermittlung derjenigen Punktverteilung, mit welcher die Zielfunktion durch eine Modellfunktion $Y(x)$ bestmöglich approximiert werden kann. Als allgemeiner Ansatz für $Y(x)$ dient (Fang et al., 2006):

$$
Y(x)=\sum_{j=1}^{k} \beta_{j} f_{j}(x)+Z(x)
$$

wobei $f_{j}(x)$ bekannte Polynomfunktionen mit den jeweils noch zu bestimmenden Koeffizienten $\beta_{j}$ darstellen und $Z(x)$ einen stochastischen Prozess abbildet. Dafür wird häufig das KRIGING-Modell verwendet, für dessen Polynomfunktionen ein linearer Ansatz gewählt wird. Der zugehörige stochastische Prozess $Z(x)$ stellt einen GAussschen Zufallsprozess mit dem Erwartungswert $E(Z(x))=0$ und der Kovarianz nach G1. 5.15 dar (Morris und Mitchell, 1995).

$$
\operatorname{Cov}\left(Z\left(f x^{(i)}\right), Z\left(x^{(j)}\right)\right)=\sigma^{2} R\left(x^{(i)}, x^{(j)}\right)
$$

Hierbei verkörpert $\sigma^{2}$ die Varianz des Approximationsfehlers und $R\left(x^{(i)}, x^{(j)}\right)$ stellt die als bekannt vorauszusetzende Korrelation dar, welche für den Fall des Kriging-Modells der GAUsSschen Korrelationsfunktion entspricht (Fang et al., 2006):

$$
R\left(x^{(i)}, x^{(j)}\right)=\exp \left\{-\theta_{k} \sum_{j=1}^{l}\left|x_{k}^{(i)}-x_{k}^{(j)}\right|^{q}\right\}, \quad 1 \leq q \leq 2
$$

In den meisten Fällen ist die Korrelationsstruktur jedoch nicht bekannt, weshalb zunächst die beiden Parameter $\theta_{k}$ und $q$ bestimmt werden müssen. Dies stellt einen deutlichen Nachteil des Ansatzes dar (Husslage, 2006).

Stellvertetend für diese Klasse steht in SPC-Opt das Maximum-Entropy-Designkriterium zur Verfügung. Es ist ursprünglich ein von Shannon (1948) vorgestelltes Maß für den Informationsgehalt einer Verteilung von Punktdaten und wurde u.a. in (Shewry und Wynn, 1987) auf das Problem der Ermittlung eines optimalen Designs angewendet. Bei Nutzung des KRIGING-Modells entspricht die Maximierung der Entropie der Maximierung der Determinante der Korrelationsmatrix $R$, welche entsprechend Gl. 5.16 berechnet wird (Fang et al., 2006). Die Optimierungsaufgabe lautet demnach:

$$
\max _{D \in \mathcal{D}} \log |R|
$$




\subsubsection{Stochastische Optimierungsverfahren}

Unabhängig vom verwendeten Designkriterium wird ein Optimierungsverfahren zur Ermittlung des optimalen Designs entsprechend Gl. 5.4 benötigt. Für praktische Aufgaben haben sich hierfür stochastische Verfahren bewährt, mit denen bei begrenztem Berechnungsaufwand gute Näherungen des global optimalen Designs gefunden werden können. Einen entsprechenden Überblick und Verweise auf entsprechende Literatur bietet z.B. (Fang et al., 2003). Von den verschiedenen Verfahren werden nachfolgend die drei in SPC-Opt implementierten Ansätze vorgestellt. Da diese lediglich eine Auswahl der verfügbaren Verfahren zur Findung optimaler Designs darstellen, sei an dieser Stelle auf den in Kap. 4 von (Landgraf, 2008) enthaltenen Überblick zu weiteren Verfahren verwiesen. Zudem werden darin Verfahren zur Erzeugung neuer Designs auf der Basis bereits vorhandener Design sowie geeignete Abbruchkriterien für die nachfolgenden Ansätze vorgestellt.

\section{Random-Search-Verfahren}

Dieses Verfahren entspricht der in Kap. 5.1 beschriebenen Monte-Carlo-Methode zur Erzeugung eines Startdesigns. Dabei wird wiederkehrend der Zielfunktionswert $\phi\left(D^{c}\right)$ des aktuellen Designs $D^{c}$ mit $\phi\left(D^{N e w}\right)$ eines zufällig neu erzeugten Designs $D^{\text {new }}$ verglichen. Falls $\phi\left(D^{n e w}\right)<\phi\left(D^{c}\right)$ gilt, wird $D^{c}=D^{n e w}$ gesetzt. Dieser Prozess wird bis zum Erreichen eines definierten Abbruchkriteriums wiederholt. Das am Ende des Prozesses aktuell beste Design $D^{c}$ wird als global optimales Design $D^{*}$ betrachtet, auch wenn es im Allgemeinen nur eine Näherung und nicht das beste Design aller $D \in \mathcal{D}$ darstellt. Da mit dem Random-SearchVerfahren keine lokale Suche erfolgt, werden meist nur schlechte Näherungen des optimalen Designs gefunden.

\section{Local-Search-Verfahren}

Eine bessere Möglichkeit stellt das heuristische Verfahren der lokalen Suche (Local-SearchVerfahren) dar. Grundsätzlich funktioniert es nach dem gleichen Schema wie das RandomSearch-Verfahren. Abweichend davon erfolgt jedoch keine zufällige Wahl des neuen Designs aus dem gesamten Raum $\mathcal{D}$, sondern nur aus der Umgebung $N\left(D^{c}\right)$ des aktuell besten Designs $D^{c}$.

Bei der Betrachtung der Verfahren zur Definition einer Umgebung $N\left(D^{c}\right)$ und der Auswahl eines aktuellen Designs $D^{c}$ genügt die Beschränkung auf die implementierten Designtypen RD, LHD und SLHD. Die Umgebung wird hierbei durch zwei Bedingungen definiert. Einerseits müssen alle Designs der Umgebung $N\left(D^{c}\right)$ eine Teilmenge aller Designs $D \in \mathcal{D}$ darstellen. Andererseits dürfen zwischen den Eigenschaften der Designs der Umgebung $N\left(D^{c}\right)$ und $D^{c}$ nur geringe Unterschiede vorliegen. Zudem wird empfohlen, eine kleine Umgebung zu definieren und damit die Wahrscheinlichkeit zum Finden eines global optimales Design zu erhöhen (Fang et al., 2006). Dies erfolgt für die aufgeführten Designtypen in nachfolgender Form: 
RD

Es wird lediglich eine Komponente der aktuellen Permutationsmatrix geändert. Dabei muss gewährleistet sein, dass der neue Wert dem Bereich $[1, k]$ entstammt und durch die Veränderung kein doppelter Punkt entsteht. Falls die Änderungsumgebung vergrößert werden soll, können z.B. alle Komponenten einer zufälligen Zeile der Permutationsmatrix geändert werden, was einer Lageänderung des Designpunkts in allen Dimensionen entspricht. Zusätzlich können auch mehrere Designpunkte parallel verändert werden.

\section{LHD}

Ergänzend zum RD muss für ein LHD die Bedingung eingehalten werden, dass die Spalten von $\Pi$ eine Permutation von $\{1, \ldots, m\}$ bleiben müssen. Aus diesem Grund erfolgt kein willkürliches Neusetzen einer Komponente, sondern eine Vertauschung von zwei Komponenten einer Spalte ("column-exchange approach") Fang et al. (2006). Eine Vergrößerung der Umgebung kann analog zum RD durch gleichzeitiges Vertauschen mehrerer Komponentenpaare einer Spalte erfolgen oder durch die Auswahl verschiedener Spalten und dem parallelen Tausch des jeweiligen Komponentenpaares.

\section{SLHD}

Als Basis dient die Vorgehensweise des LHD. Es muss jedoch gesichert werden, das die Symmetrie des Designs erhalten bleibt (Ye et al., 2000). Dies erfolgt, indem für jeden Tausch eines Komponentenpaares auch das korrespondierende symmetrische Komponentenpaar getauscht wird. Notwendig ist dies nur dann nicht, wenn die Vertauschung bereits $\pi_{i}$ und $\pi_{n+1-i}$ betrifft. Bei SLHD mit einer ungeraden Anzahl Designpunkte ist die Vertauschung des Punktes im Zentrum nicht gestattet.

Bei der Nutzung des Local-Search-Verfahrens ist zu beachten, dass in Abhängigkeit des Startdesigns nicht die globale Lösung, sondern das nächstgelegene optimale Design gefunden wird. Diese stellt jedoch gewöhnlich nur eine lokal optimale Lösung dar.

\section{Simulated Annealing}

Eine Erweiterung des Local-Search-Verfahrens stellt der Algorithmus des Simulated Annealing (SA) dar. Es gehört zur Klasse der Metaheuristiken und wird auch als "Verfahren des simulierten Ausglühens" bezeichnet. Diesem Begriff entsprechend basiert das Grundprinzip auf dem physikalischen Prozess der langsamen Abkühlung eines Körpers vom flüssigen zum festen Zustand und wurde von Kirkpatrick et al. (1983) vorgestellt. Analog zum LocalSearch-Verfahren wird in der Umgebung eines aktuellen Designs gesucht und ein Design mit besserem Zielfunktionswerten stets übernommen. Allerdings können hier entsprechend eines Zufallsalgorithmus auch schlechtere Designs als das aktuelle akzeptiert werden, womit das Verlassen eines bereits gefundenen Minimums ermöglicht wird. Die Wahrscheinlichkeit $\pi_{S A}$ für diese Akzeptanz ergibt sich zu:

$$
\pi_{S A}=\exp \left(-\frac{\phi\left(D^{c}\right)-\phi\left(D^{\text {new }}\right)}{T}\right)
$$


Daraus geht hervor, dass die Akzeptanzwahrscheinlichkeit umso höher ist, desto kleiner die Differenz zwischen dem aktuellen und dem neuen Design ist. Der Prozessparameter $T$ wird in Anlehnung an den physikalischen Prozess als Temperatur bezeichnet und verringert sich, ausgehend von einem Startwert $T_{0}$, iterativ mit jeder neuen Suche. Die Abnahme von $T$ wird durch einen Abkühlungsfaktor $\alpha$ gesteuert, wodurch sich $T$ im k-ten Schritt entsprechend

$$
T_{k}=T_{k-1} \cdot \alpha ; \quad 0<\alpha<1
$$

ergibt. Da für alle schlechteren Designs $\phi\left(D^{c}\right)>\phi\left(D^{\text {new }}\right)$ gilt, kann $\pi_{S A}$ entsprechend Gl. 5.18 Werte zwischen 0 und 1 annehmen. Die Übernahme des schlechteren Designs erfolgt immer dann, wenn $\pi_{S A}$ größer als ein zufälliger Wert aus einer gleichverteilten Menge $U[0,1]$ ist, d.h. wenn das Kriterium

$$
p\left(T, D^{c}, D^{\text {new }}\right)=\left\{\begin{array}{ll}
\text { true } & \text { falls } \\
\text { false } & \text { sonst }
\end{array} \pi_{S A} \geq u ; \quad u \in U[0,1]\right.
$$

den Wert "true" zurück gibt.

Da $S P C$-Opt verschiedene Designkriterien enthält und dabei unterschiedliche Größenordnungen für $\phi(D)$ und $\nabla \phi$ auftreten, ist eine Normierung der Starttemperatur $T_{0}$ erforderlich. Dies erfolgt durch die Erzeugung einer bestimmten Anzahl Designpaare $D^{c}$ und $D^{\text {new }} \in N\left(D^{C}\right)$ zu Beginn des Algorithmus' und die Bestimmung der zugehörigen Differenzen $\nabla \phi$. Der Mittelwert $\nabla \phi_{\text {average }}$ aller Differenzen wird zur Skalierung von $T_{0}$ nach

$$
\left(T_{0}\right)_{\text {scale }}=T_{0} \cdot \nabla \phi_{\text {average }}
$$

verwendet. Zur effektiven Suche mit dem SA-Verfahren müssen geeignete Werte für $T_{0}$ und $\alpha$ gewählt werden. Bei zu hoher Starttemperatur oder zu großem Abkühlungsfaktor konvergiert der Algorithmus nur langsam. Werden die Prozessparameter jedoch zu niedrig gewählt, so erfolgt keine effiziente globale Suche und das Verfahren geht frühzeitig in eine lokale Suche über. 


\section{Identifikation von Materialparametern anhand bauteilnaher Probekörper}

\subsection{Problemstellung}

Gummiwerkstoffe finden aufgrund ihrer besonderen Materialeigenschaften in vielen technischen Produkten Verwendung. Dazu gehören beispielsweise Reifen, elastische Aufhängungen, Transportbänder, Ummantelungen bzw. Isolationen sowie eine breite Palette Dichtungs-, Dämpfer- und Lagerelemente. Aufgrund steigender Anforderungen an diese Bauteile und reduzierter Entwicklungszeiten sind FEM-Simulationen auch in diesem Bereich der Produktentwicklung praktisch unverzichtbar geworden. Die Aussagekraft der Simulationen wird jedoch durch die Eignung sowohl der verwendeten Stoffgesetze als auch der zugeordneten Materialparameter limitiert. Insbesondere das inelastische Materialverhalten, welches zu charakteristischen Effekten wie z.B. Hysterese, Entfestigung oder bleibender Verformung führt, hat im Bereich der Materialmodellierung mittlerweile zu einer Reihe hochwertiger Stoffgesetze geführt. Beispiele dafür sind unter anderem die Arbeiten von Dargazanv und Itskov (2009), Lion (1996), Klüppel und Schramm (2000) und Besdo und Ihlemann (2003).

Die Identifikation von Stoffparametern erfolgt im allgemeinen anhand von standardisierten Laborprüfkörpern und ist bereits Stand der Technik (Hohl, 2007). Die Probekörper sind meist durch eine sehr einfache Geometrie gekennzeichnet und die Beanspruchungen können als näherungsweise homogen charakterisiert werden. Die entsprechenden Experimente liefern deshalb Informationen über den direkten Zusammenhang von Spannungen und Verzerrungen. Demzufolge kann das Materialverhalten unmittelbar charakterisiert werden und die notwendige Rechenkapazität ist sehr gering. Die Identifikation der Materialparameter erfolgt gewöhnlich durch die Lösung eines Fehlerquadratminimumproblems (Luenberger, 1997).

Technische Bauteile und zugehörige Laborprüfkörper haben in der Regel sehr verschiedene Geometrien und werden zudem häufig in unterschiedlicher Weise hergestellt. Dies bedingt in vielen Fällen gravierende Abweichungen im Materialverhalten. Bauteilsimulationen mit Stoffgesetzen, die an Messungen an solchen Prüfkörpern angepasst wurden, sind somit bereits von vornherein fehlerbehaftet. Die Beschränkung auf homogene Referenzmessungen führt zu zusätzlichen Einbußen bei der Qualität und der Verlässlichkeit der Stoffgesetzanpassung. Einerseits ist die Zahl der realisierbaren, näherungsweise homogenen Belastungsverteilungen gering (gerade die häufig ausgeführte einachsige Zugbelastung tritt in technischen Bauteilen kaum auf). Zudem bleiben die zwangsläufigen Abweichungen von der Homogenität gänzlich unberücksichtigt und in ihrer Ausprägung weitgehend unbekannt. Insbesondere letzteres ist häufig, z.B. beim Druckversuch, eine Folge unklarer Randbedingungen.

Ein Lösungsansatz, welcher im Projekt "Stoffgesetzanpassung anhand bauteilnaher Probekörper" umgesetzt wurde, besteht in der effizienten Verwendung von Messdaten aus Versu- 
chen an bauteilnahen Prüfkörpern mit inhomogen verteilten Spannungen und Verzerrungen zur Identifikation von Materialparametern. Auf diesem Weg werden die genannten Nachteile der Beschränkung auf homogene Referenzmessungen vermieden und es eröffnet sich die Möglichkeit, spezifische Besonderheiten von Produktgruppen und Belastungsprozessen bei der Anpassung der Stoffgesetze zu berücksichtigen. Die für die Referenzdaten untersuchten Prüfkörper sollten den später zu simulierenden Bauteilen ähneln, aber möglichst einfach zu modellieren sein. Zudem sollten die Randbedingungen sowohl experimentell als auch in der virtuellen Nachbildung eindeutig und mit hoher Genauigkeit einstellbar bzw. erfassbar sein. Mit diesem Ansatz geht unweigerlich eine Erhöhung der Rechenzeiten einher. Dies ist beim Leistungsumfang heutiger Standardcomputer jedoch von untergeordneter Bedeutung, sofern das Potential effizienter Algorithmen und geschickter Programmierung voll ausgeschöpft wird.

\subsection{Bauteilgeometrie und Belastungen}

Die Umsetzung des gewählten Lösungsansatzes erfolgt im Rahmen eines durch verschiedene Firmen finanzierten Forschungsvorhabens anhand eines konkreten Bauteils. Dabei handelt es sich um eine Fahrwerksbuchse aus der Serienproduktion der Anvis Deutschland GmbH, welche für das Projekt adaptiert wurde. Diese besteht aus einer metallischen Innen- und Außenbuchse sowie einer dazwischen liegenden Gummischicht.
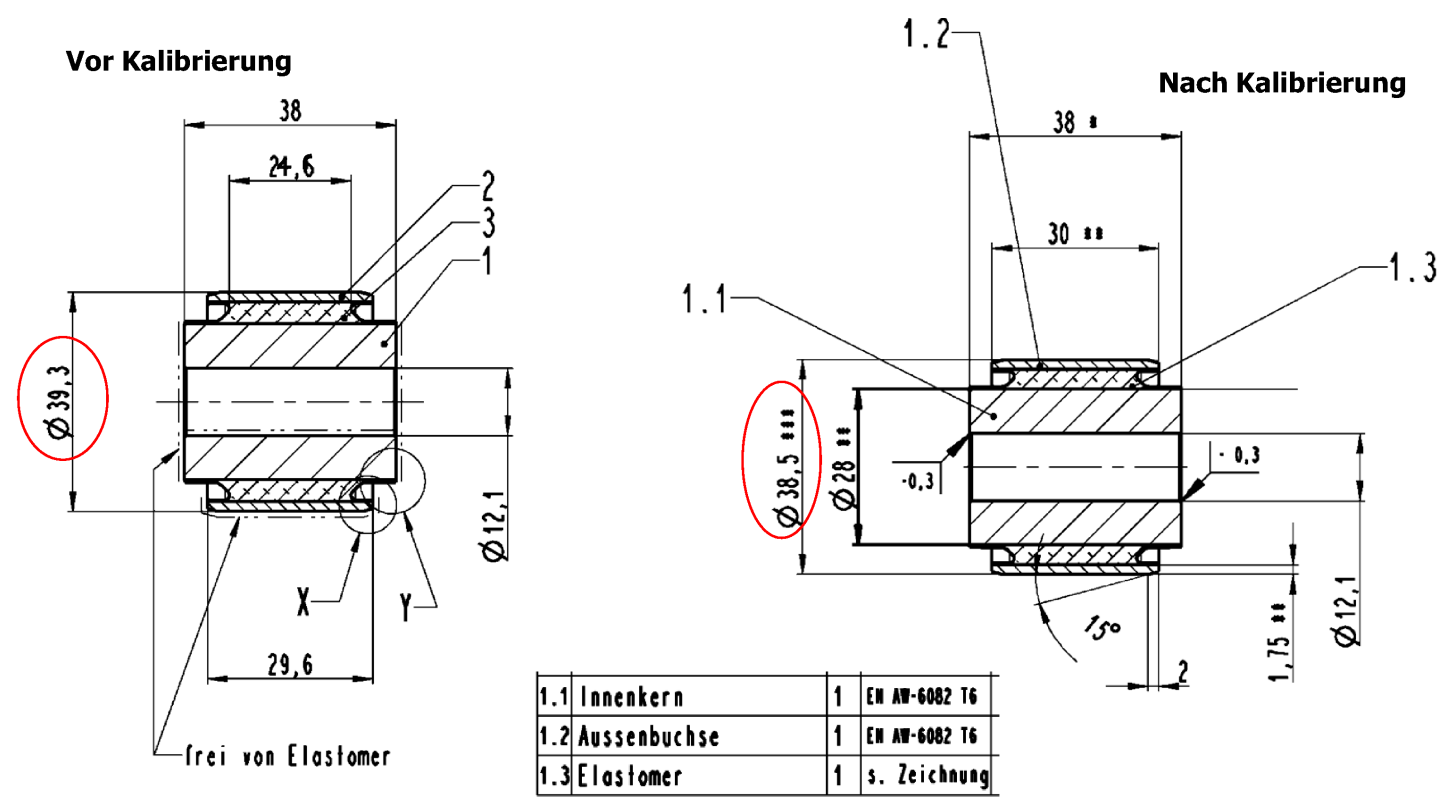

Bild 6.1: Technische Zeichnung der adaptierten Fahrwerksbuchse.*

Die Adaption der Buchse besteht darin, dass die eigentlich konturierte Innenhülse durch eine gerade Hülse mit einem Außendurchmesser von $d=28 \mathrm{~mm}$ ersetzt (Bild 6.1) wird. Der Innendurchmesser der äußeren Hülse beträgt $D=35,8 \mathrm{~mm}$ und die Länge der Gummischicht $L=24,6 \mathrm{~mm}$. Dies resultiert in einem Formfaktor $s=2 L /(D-d) \approx 6,3$, welcher aufgrund

*Anvis Deutschland GmbH 
seiner Höhe charakteristisch für eine dünne Gummischicht ist. Die Herstellung der Buchse erfolgt mit Hilfe eines bereits vorhandenen Prototypenwerkzeugs. Nach dem Abkühlprozess und der abgeschlossenen Vulkanisation verbleiben aufgrund der thermischen und chemischen Schrumpfung im Inneren der Gummischicht Eigenspannungen, welche zu hydrostatischen Zugspannungsanteilen führen. Da dies negative Auswirkungen auf die Dauerfestigkeit der Buchse hat, wird die Buchse in einer entsprechenden Kalibriervorrichtung, welche ebenfalls zur Verfügung steht, in Umfangsrichtung gestaucht. Während dieses Kalibriervorgangs werden die vorhandenen Zugspannungen nicht nur ausgeglichen, sondern sogar überkompensiert. Bild 6.2 zeigt die adaptierte Fahrwerksbuchse im kalibrierten Zustand.

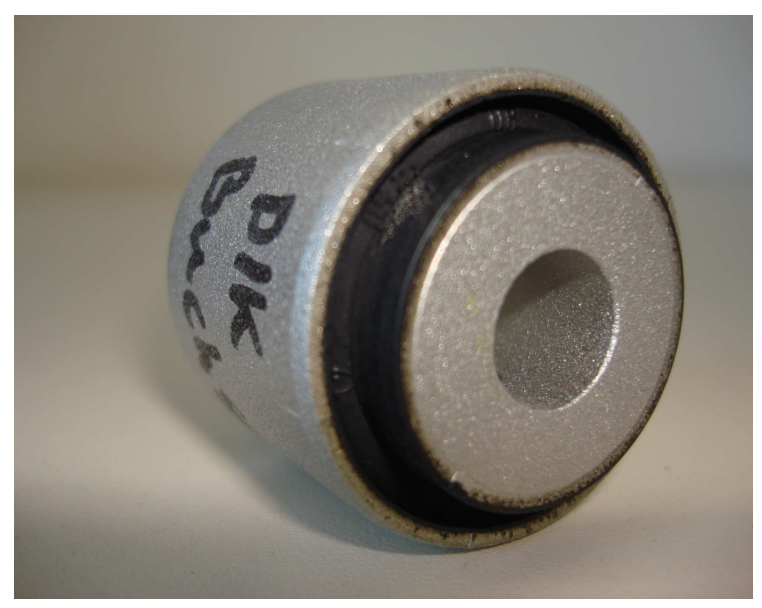

Bild 6.2: Kalibrierte Fahrwerksbuchse.

Bei der Erstellung des zugehörigen FE-Modells werden die CAD-Daten der Fahrwerksbuchse zugrunde gelegt (Bild 6.3). Die Abweichungen von der Rotationssymmetrie können für die Modellierung des 3D-Modells vernachlässigt werden. Ein entsprechender Meridianschnitt durch die Gummischicht ist in Bild 6.4 dargestellt.
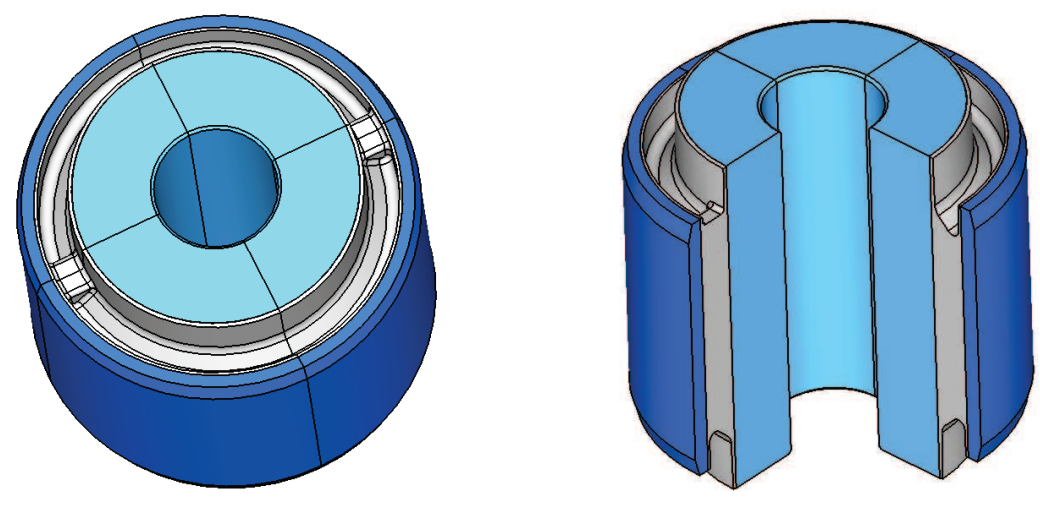

Bild 6.3: CAD-Modell der Fahrwerksbuchse.

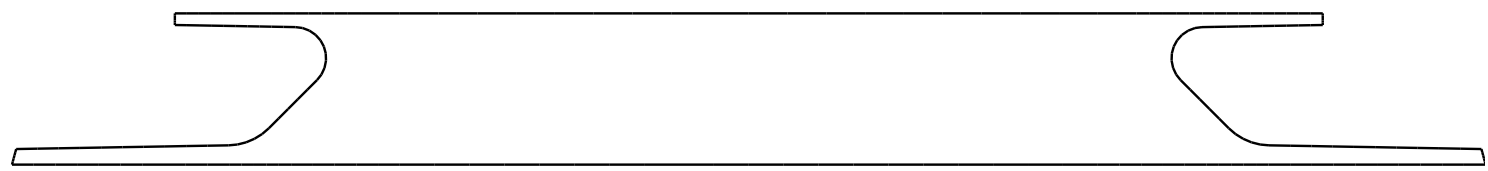

Bild 6.4: Meridianschnitt der Gummischicht der Fahrwerksbuchse. 
Für die originale Fahrwerksbuchse sind im Lastenheft typische Belastungsarten sowie die zugehörigen Amplituden bereits vorgegeben. In den entsprechenden Versuchen am Bauteil werden diese jeweils in einem Belastungszyklus aufgebracht. Dieser wird solange wiederholt, bis ein quasistationärer Zustand erreicht ist. Die im Lastenheft definierten Beanspruchungen des Bauteils werden im Folgenden vorgestellt.

\section{Axiale Belastung}

Die axiale Belastung stellt den einfachsten Lastfall dar. Dabei wird das Bauteil in axialer Richtung mit einer Maximalkraft von $F^{\text {axial }}=1 \mathrm{KN}$ belastet (Bild 6.5).

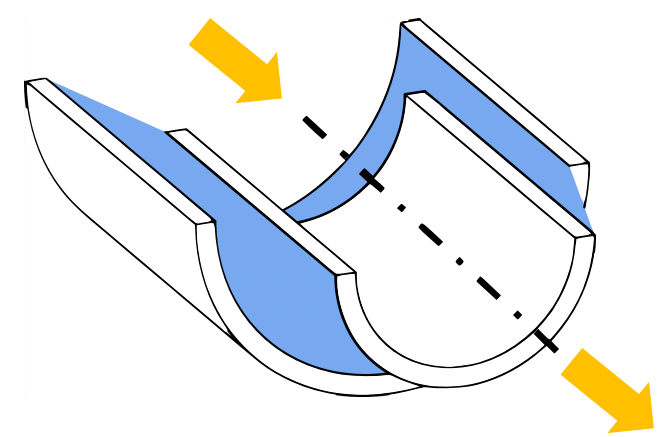

(a) Schematische Darstellung

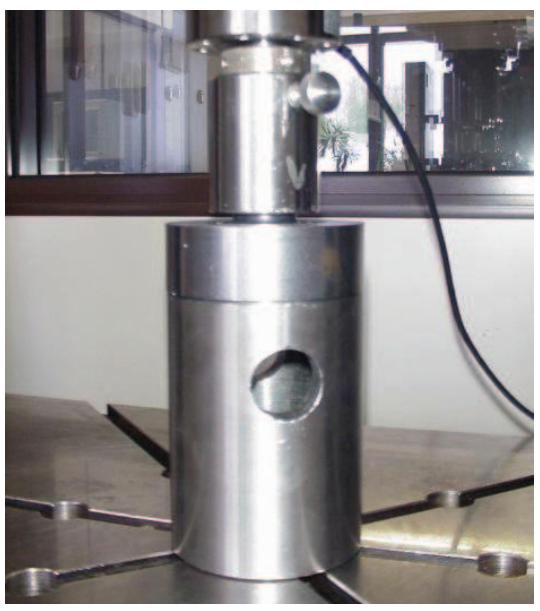

(b) Versuchsaufbau*

Bild 6.5: Axiale Belastung.

\section{Radiale Belastung}

Bei der radialen Belastung wird das Bauteil in radialer Richtung mit einer Maximalkraft von $F^{\text {rad }}=10 \mathrm{KN}$ belastet. Das zugehörige Belastungsschema sowie der Versuchsaufbau sind in Bild 6.6 dargestellt.

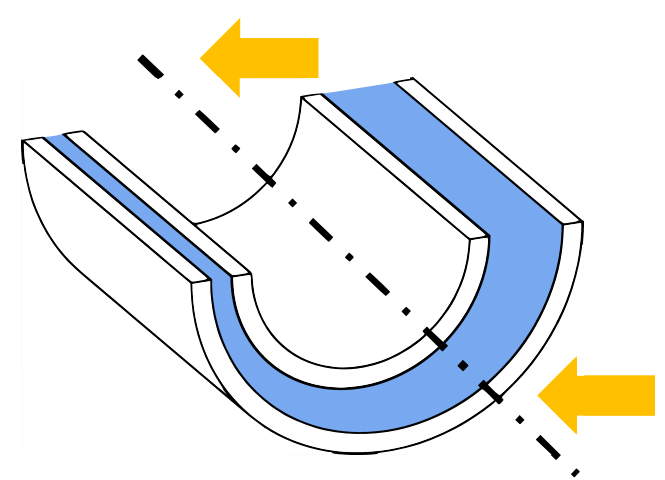

(a) Schematische Darstellung

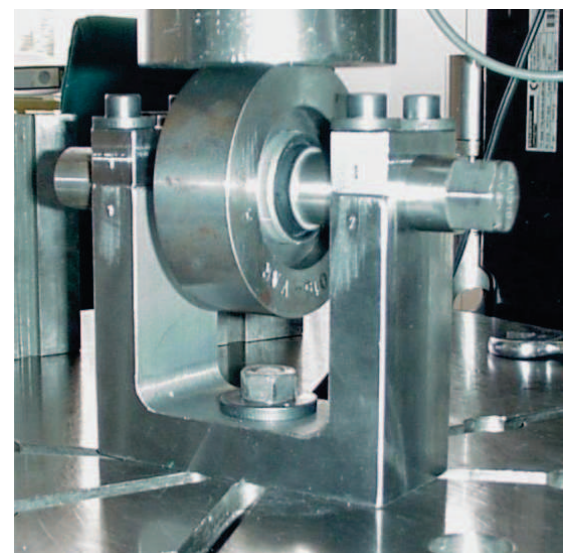

(b) Versuchsaufbau*

Bild 6.6: Radiale Belastung.

*Anvis Deutschland GmbH 


\section{Kardanische Belastung}

Das Belastungsschema und der Versuchsaufbau für die kardanische Belastung sind in Bild 6.7 dargestellt. Der Maximalwinkel der Verdrehung für das Bauteil beträgt $\varphi^{\text {kard }}=3^{\circ}$.

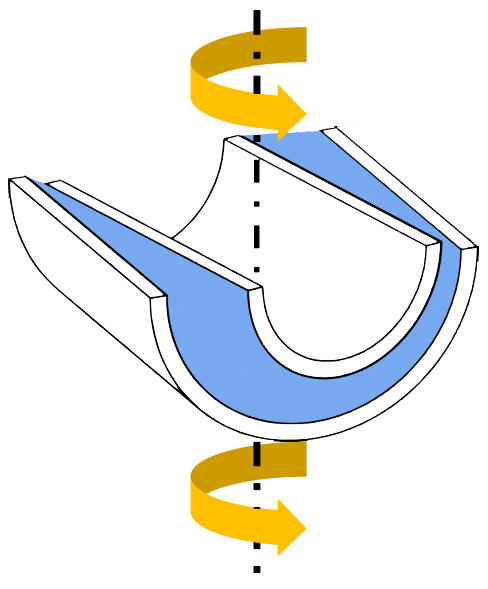

(a) Schematische Darstellung

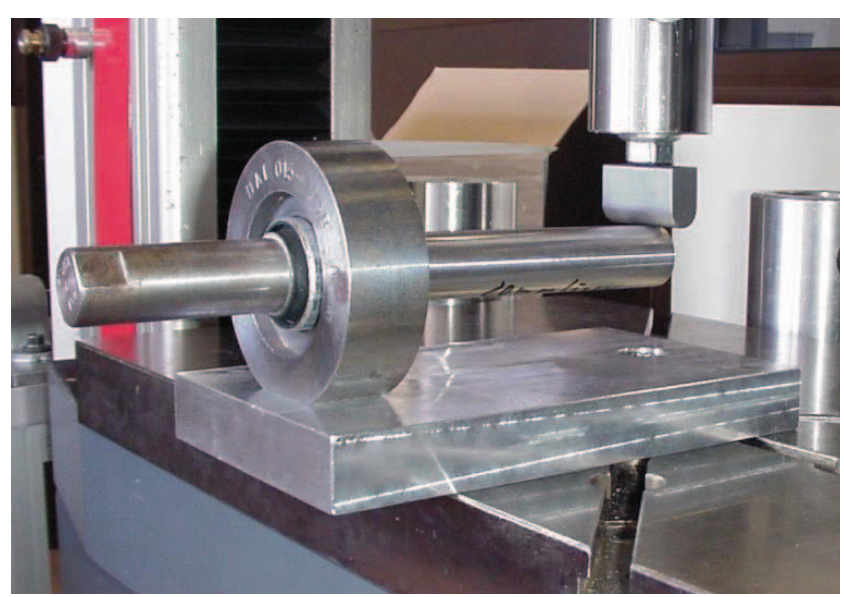

(b) Versuchsaufbau*

Bild 6.7: Kardanische Beanspruchung.

\section{Torsionsbelastung}

Bei der Torsionsbelastung wird die Innenbuchse gegenüber der Außenbuchse in axialer Richtung um einen Maximalwinkel von $\varphi^{\text {Tors }}=15^{\circ}$ verdreht (Bild 6.8).

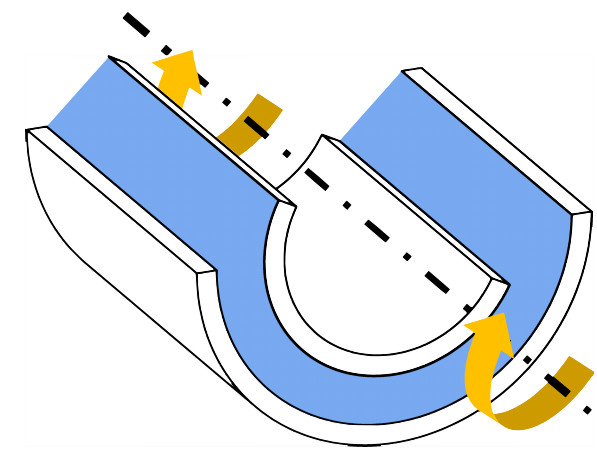

(a) Schematische Darstellung

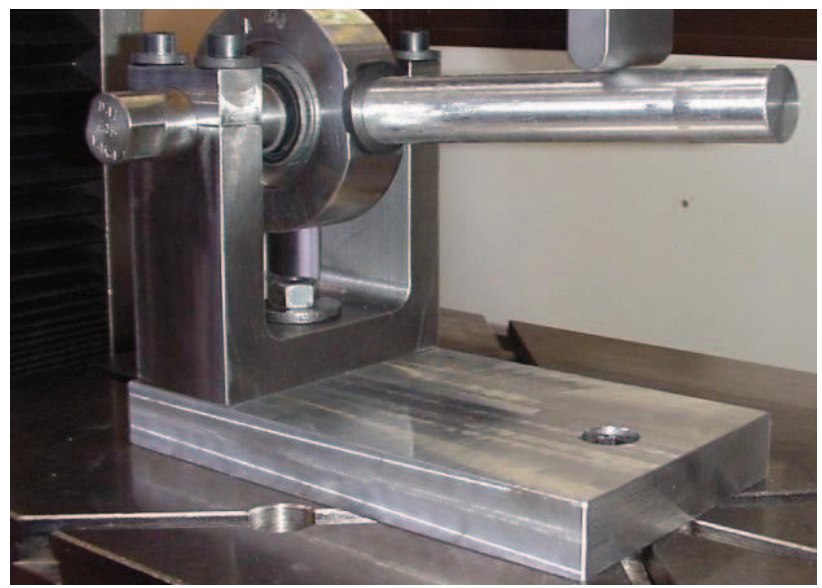

(b) Versuchsaufbau*

Bild 6.8: Torsionsbeanspruchung.

Die Darstellung der verschiedenen Belastungen erfolgt in entsprechenden FEM-Simulationen, für welche neben der Erstellung des FE-Modells auch die Auswahl eines geeigneten Stoffgesetzes vorgenommen werden muss. Hierfür ist jedoch die genauere Betrachtung der für die Fahrwerksbuchsen verwendeten Gummimischung notwendig. 


\subsection{Gummimischung}

Der hauptsächliche Bestandteil bei der Herstellung von Elastomeren ist entweder Naturkautschuk (NR) oder ein Synthesekautschuk. Letzteres umfasst eine ganze Reihe verschiedener Gummimischungen, welche sich in ihren Bestandteilen und Eigenschaften sehr stark voneinander unterscheiden, z.B. Styrol-Butadien-Kautschuk (SBR) oder Ethylen-PropylenDien-Kautschuk (EPDM). Weiterhin beinhalten Elastomere diverse Zusätze wie Füllstoffe, Aktivatoren, Beschleuniger oder Haftmittel, welche vor der Vulkanisation mit dem Kautschuk zur Elastomermischung (elastomer compound) verarbeitet werden. Bezüglich eines Überblicks zu den verschiedenen Gummimischungen und zugehörigen Herstellungsprozessen sei an dieser Stelle auf (Röthemeyer und Sommer, 2001; Hofmann und Gupta, 2010; Nagdi, 2004) und (Elias, 2009) verwiesen.

Das Verhalten eines Bauteils hängt demnach maßgeblich von der gewählten Gummimischung ab. Für das Materialverhalten ist jedoch nicht nur die chemische Zusammensetzung, sondern auch der Herstellungsprozess entscheidend. Wie im betrachteteten Beispiel unterscheiden sich Geometrie und Herstellungsprozess von technischen Bauteilen und den zugehörigen Laborprüfkörpern in der Regel deutlich voneinander. Dies bedingt trotz gleicher Gummimischung deutliche Abweichungen im Materialverhalten, welche sich für ein gewähltes Stoffgesetz in Form jeweils unterschiedlicher Materialparameter manifestieren.

Tabelle 6.1: Chemische Zusammensetzung der verwendeten Gummimischung.

\begin{tabular}{|c|c|}
\hline \multicolumn{2}{|c|}{ NR-Mischungsvariation N2M } \\
\hline Komponente & pphr \\
\hline \multicolumn{2}{|c|}{ Grundmischung } \\
\hline NR (TSR CV 60) & 100 \\
\hline Ruß N-33024 & $24 / 28$ \\
\hline IPPD & 2 \\
\hline TMQ & 1 \\
\hline Antilux 500 & 2 \\
\hline $\mathrm{ZnO} \mathrm{RS}$ & 5 \\
\hline Stearinsäure & 2 \\
\hline \multicolumn{2}{|c|}{ Vernetzungssystem } \\
\hline CBS & 2 \\
\hline Schwefel & 1,5 \\
\hline TMTD & 0,2 \\
\hline
\end{tabular}

Um die Ergebnisse der Identifikation auf Basis von Standardprobekörpern mit denen der Identifikation anhand bauteilnaher Probekörper vergleichen zu können, wurde die Gummimischung für Standardprobekörper, bauteilnahe Probekörper und Bauteile nach dem gleichen Rezept und in einer Charge hergestellt. Die Materialeigenschaften orientieren sich beim betrachteten Bauteil an denen derjenigen Gummimischung, welche normaler Weise zur Herstellung der Fahrzeugbuchsen bei der Anvis Deutschland GmbH verwendet wird. Es handelt 
sich dabei um einen mit Ruß gefüllten Naturkautschuk, dessen chemische Zusammensetzung in Tabelle 6.1 dargestellt ist.

Bei Lagerung einer fertigen, jedoch unvulkanisierten, Kautschukmischung verändern sich im Laufe der Zeit deren Eigenschaften. Um dies zu vermeiden, wurde die fertige Kautschukmischung nach der Herstellung zeitnah verarbeitet - sowohl für die Standardprobekörper als auch für die Bauteile und bauteilnahen Probekörper. Temperatur und Dauer der Vulkanisierung wurden auf Basis der in Bild 6.9] dargestellten Vulkameterkurve gewählt.

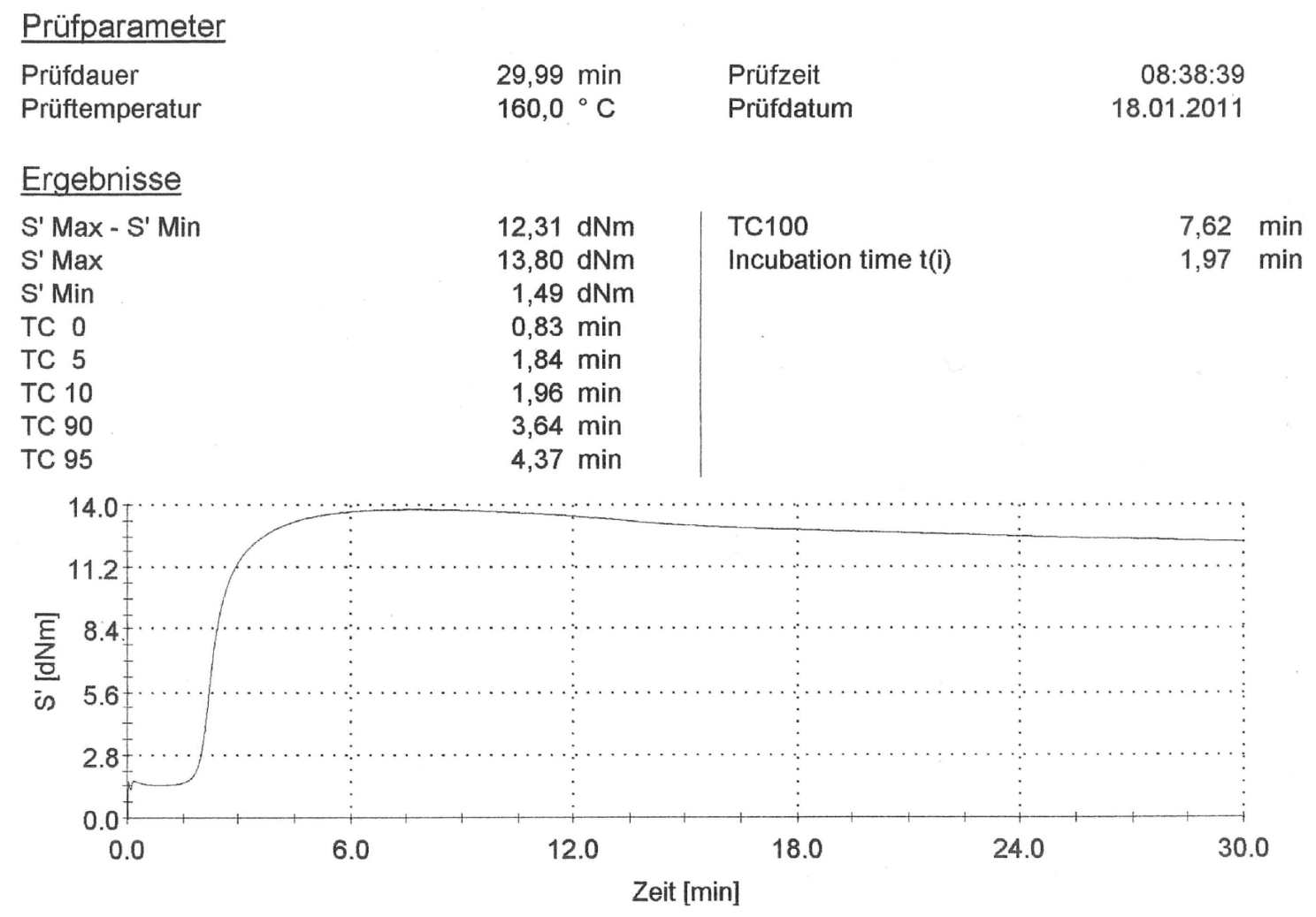

Bild 6.9: Vulkameterkurve, aufgenommen mit Rheometer MDR 2000 E.

\subsection{Stoffgesetze}

In der industriellen Anwendung hat ein geeignetes Materialmodell praktisch nie die Aufgabe, das reale Werkstoffverhalten möglichst genau und vollständig nachzubilden. Im Vordergrund für die Praxistauglichkeit eines Stoffgesetzes steht vielmehr der ausgewogene Kompromiss zwischen problemspezifischen Anforderungen an Geltungsbereich, Genauigkeit und Eigenschaftskombination der Materialbeschreibung auf der einen Seite und wirtschaftlichen Beschränkungen bezüglich erforderlicher Computerkapazitäten, Berechnungszeiten und Charakterisierungsaufwand auf der anderen Seite. Für FEM-Simulationen von ElastomerBauteilen werden deshalb vorwiegend hyperelastische Materialgesetze genutzt, welche in den meisten kommerziellen FEM-Programmen verfügbar sind. Kennzeichnend ist die ausschließliche Abhängigkeit des Spannungszustands von der aktuellen Deformation und nicht von deren Vorgeschichte, wodurch die Darstellung von Hysterese-Effekten unmöglich ist. Die Abbildung des realen Materialverhaltens ist dadurch zwar begrenzt, im Gegenzug ist die Zahl der Materialparameter aber gering. Dies ist insbesondere im Hinblick auf die notwen- 
digen FEM-Simulationen für gestörte Parameter im Rahmen einer Identifikation günstig. Ein weiterer Vorteil ist das bessere Konvergenzverhalten gegenüber mächtigeren, und damit einhergehend meist komplexeren, Materialmodellen.

Da es bislang in vielen Aspekten noch nicht gelungen ist, die tatsächlichen physikalischen Vorgänge innerhalb von Elastomeren in entsprechenden Stoffgesetzen umzusetzen, wird das Materialverhalten meist rein phänomenologisch nachgebildet. Darüber hinaus wird gewöhnlich inkompressibles Materialverhalten angenommen. Für die im Folgenden vorgestellten Stoffgesetze wird die Formulierung der Energiedichte $\hat{W}$ in Abhängigkeit der Hauptinvarianten des Rechts-CAUCHY-GREEN-Tensors verwendet.

Das einfachste hyperelastische Stoffgesetz ist das NEO-HoOKEsche Gesetz, welches in inkompressibler Formulierung nur einen Parameter $\alpha$ beinhaltet:

$$
\hat{W}=\alpha\left(I_{1}-3\right) \quad \text { mit: } \quad I_{3} \stackrel{!}{=} 1 .
$$

Es stellt eine Sonderform des Mooney-Rivlin-Ansatzes (Mooney, 1940) dar, welcher zusätzlich einen von $I_{2}$ abhängigen Term und somit einen weiteren Parameter $\beta$ besitzt:

$$
\hat{W}=\alpha\left(I_{1}-3\right)+\beta\left(I_{2}-3\right) \quad \text { mit: } \quad I_{3} \stackrel{!}{=} 1 .
$$

Um die Nichtlinearität besser abbilden zu können, kann das YEOH-Stoffgesetz (Yeoh, 1990) genutzt werden, welches eine Erweiterung des NEO-HoOKE-Gesetzes um zwei weitere von $I_{1}$ abhängige Terme darstellt:

$$
\hat{W}=\alpha_{1}\left(I_{1}-3\right)+\alpha_{2}\left(I_{1}-3\right)^{2}+\alpha_{3}\left(I_{1}-3\right)^{3} \quad \text { mit: } \quad I_{3} \stackrel{!}{=} 1 .
$$

Im Gegensatz zu den vorgenannten Ansätzen ist das OGDEN-Gesetz (Ogden, 1972) auf Basis der Hauptstreckungen $\lambda_{i}$ formuliert:

$$
\hat{W}=\sum_{n=1}^{N} \frac{\mu_{n}}{\alpha_{n}}\left(\lambda_{1}^{\alpha_{n}}+\lambda_{2}^{\alpha_{n}}+\lambda_{3}^{\alpha_{n}}-3\right) \text { mit: } I_{3} \stackrel{!}{=} 1 .
$$

Die Anzahl der Materialparameter $\alpha_{n}$ und $\mu_{n}$ kann durch den Anwender festgelegt werden. Bei passender Wahl von $\alpha_{n}$ geht das OGDen-Gesetz in das NeO-Hookesche Gesetz $\left(N=1, \alpha_{1}=2\right)$ bzw. in den Mooney-Rivlin-Ansatz $\left(N=2, \alpha_{1}=2, \alpha_{2}=-2\right)$ über. Beim Setzen der Parameter ist zu beachten, dass die Verwendung mehrerer Potenz-Terme zwar die Anpassung an ein Experiment verbessert, die damit einhergehende steigende Parameterzahl jedoch zu ungewünschten Korrelationen zwischen diesen führen kann (Kap. 6.6.4). Zudem ist für eine zuverlässige Bestimmung der Parameter eine umfangreichere Basis experimenteller Daten notwendig.

Weitere, an dieser Stelle nicht näher betrachtete, hyperelastische Stoffgesetze stammen z.B. von Gent und Thomas (1958), Hart-Smith (1966) und Alexander (1968). Aufgrund ihrer Verfügbarkeit in den üblichen FEM-Programmen (hier: $M S C . M A R C^{*}$ und $A B A Q U S^{*}$ ) sowie der guten Praxistauglichkeit werden für das vorliegende Beispiel die Ansätze von YEOH und OGDEN verwendet.

\footnotetext{
*Detaillierte Angaben sind im Anhang A.1 aufgeführt.
} 
Ein entscheidender Nachteil der hyperelastischen Stoffgesetze besteht in der sehr begrenzten Abbildungsmöglichkeit typischer Effekte von Elastomeren. In den vergangenen Jahren wurden deshalb verschiedene inelastische Ansätze entwickelt, um einige dieser Effekte beschreiben zu können. Für die in der Fahrzeugbuchse verwendete NR-Mischung sind vor allem Entfestigung, Hystereseerscheinungen sowie verbleibende Restverformungen von Bedeutung. Zur Verdeutlichung sind in Bild 6.10 die Ergebnisse eines mehrstufigen uniaxialen Zugversuches an einem S2-Zugstab aus dieser NR-Mischung dargestellt.
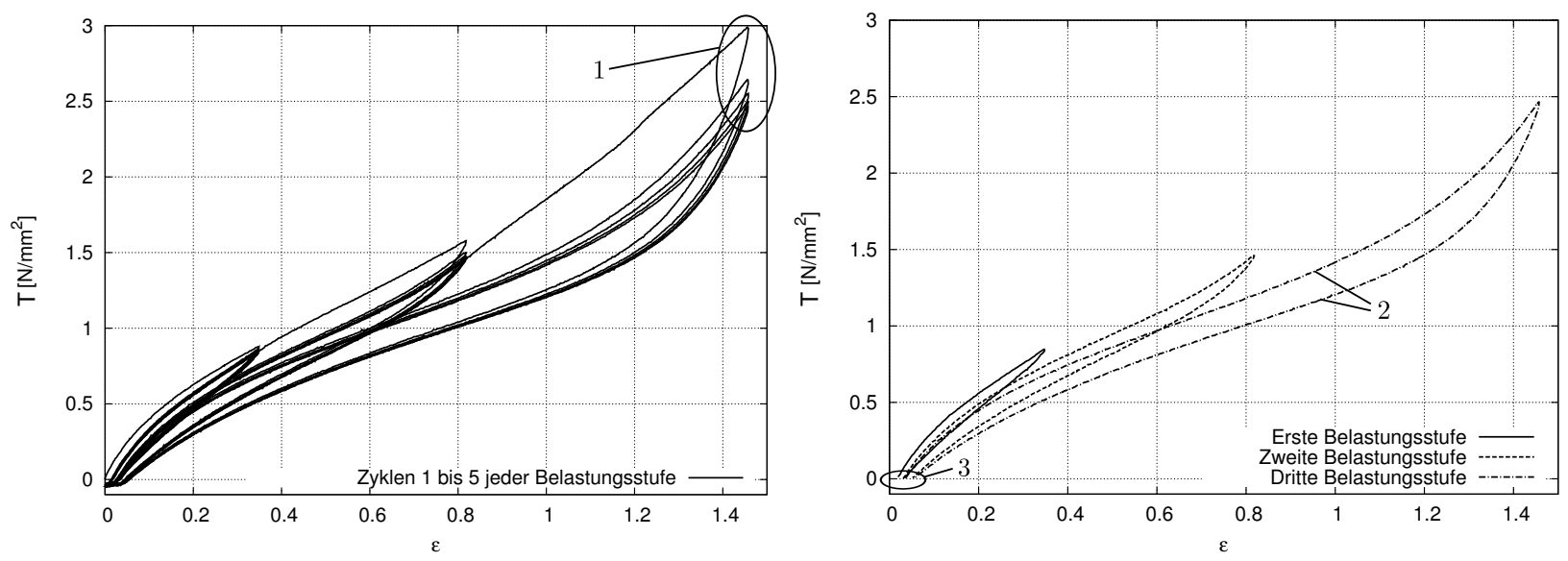

Bild 6.10: Spannungs-Dehnungs-Verlauf für einen mehrstufigen einachsigen S2-Zugversuch mit jeweils 5 Zyklen (links) sowie der für die Identifikation verwendeten (quasi)stationären Zyklen (rechts). Zusätzlich Kennzeichnung der Entfestigung durch Lastwiederholung (1), Hysterese (2) und Restverformung (3).

Infolge von wiederholten Lastamplituden kommt es zu der für Gummi typischen Entfestigung, welche auch als Mullins-Effekt bezeichnet wird. Einen weiteren Mullins-Effekt stellt die Vorreckung eines Materials dar, welchem vor allem die verbleibende Restverformung zugeordnet werden kann. Zur Abbildung dieser Effekte in einem Materialmodell existieren verschiedene Ansätze, z.B. von Klüppel und Schramm (2000), Miehe et al. (2004), Miehe und Göktepe (2005) oder Besdo und Ihlemann (2003). Weiter entwickelte Stoffgesetze bilden darüber hinaus auch die belastungsinduzierte Anisotropie (Göktepe und Miehe, 2005; Dargazany und Itskov, 2009; Freund und Ihlemann, 2010) ab. Obwohl die Entfestigungserscheinungen zumindest teilweise reversibel sind, spielt dies bei der Wahl des Materialmodells für die betrachtete Fahrwerksbuchse aufgrund der langen Erholungszeit keine Rolle.

Vor allem gefüllte Gummiwerkstoffe wie die verwendete NR-Mischung weisen teils erhebliche Hystereseerscheinungen auf. Der damit verbundene Unterschied zwischen Be- und Entlastung ähnelt viskoelastischem Materialverhalten. Dies spiegeln auch einige Ansätze, z.B. von Bergström und Boyce (1998) oder Lion (2000), wider. Für die hier betrachtete Anwendung steht nicht der Einspielvorgang, sondern vor allem der quasistationäre Belastungszustand im Vordergrund. Parallel dazu müssen bei der Identifikation anhand bauteilnaher Probekörper (Kap. 6.7) viele FEM-Simulationen durchgeführt werden, weshalb die Reduzierung der Anzahl simulierter Belastungszyklen außerordentlich wichtig ist. Demnach sind vor allem diejenigen Materialmodelle interessant, bei denen sich möglichst schnell eine Gleichgewichtshysterese einstellt. 
Eine weitere Eigenschaft von Elastomeren ist Relaxation als Sonderform der Viskoelastizität. In welcher Form sich die Materialspannung in gefüllten Gummiwerkstoffen unter einer vorgegebenen und konstant gehaltenen Deformation verringert, wird zum Beispiel in den Arbeiten von Gent (1962), Lion (1997) und Bergström und Boyce (1998) betrachtet. Die im Lastenheft für die betrachtete Fahrwerksbuchse definierten Belastungen werden jedoch in einem Zeitraum aufgebracht, welcher deutlich unterhalb typischer Relaxationszeiten liegt. Weiterhin würden Terme eines inelastischen Stofgesetzes, welche das relaxierende Verhalten beschreiben, die Anzahl der zu identifizierenden Parameter erhöhen. Darüber hinaus müssten zusätzliche Versuche definiert werden, anhand derer diese Parameter identifiziert werden können. Bei der Wahl des im Projekt genutzten Stoffgesetzes wird deshalb auf die Darstellungsmöglichkeit von Relaxation verzichtet.

Bei der Wahl eines geeigneten Stoffgesetzes ist nicht nur die Möglichkeit zur Abbildung typischer Gummieigenschaften relevant, sondern im Hinblick auf die industrielle Anwendung auch die Verfügbarkeit in den verwendeten FEM-Programmen MSC.MARC und ABAQUS und ein möglichst robustes Konvergenzverhalten. Leider ist eine gleichzeitige Erfüllung aller Kriterien zum aktuellen Zeitpunkt mit den eingebauten Stoffgesetzen nur mit Abstrichen möglich. Beispielsweise ist der Ansatz von Ogden und Roxburgh (1999) recht robust, umfasst wenige Materialparameter und steht als implementiertes Materialmodell bereits zur Verfügung, jedoch können damit die Hysterese-Effekte nicht abgebildet werden. Die Möglichkeit zur Überlagerung herkömmlicher hyperelastischer Materialmodelle mit viskoelastischen Termen ist nicht praktikabel, da der damit einhergehende Einfluss der Belastungsgeschwindigkeit mit den verfügbaren Experimenten nicht ausreichend berücksichtigt werden kann. Da moderne FEM-Programme jedoch die Nutzung eigener Stoffgesetze mit Hilfe von Nutzerschnittstellen erlauben (Kap. 2.4), liegt die Wahl eines modernen, noch nicht standardmäßig implementierten, Stoffgesetzes nahe. Üblicherweise werden in der Literatur bereits identifizierte Parametersätze für die vorgestellten inelastischen Materialmodelle angegeben. Es existieren jedoch selbst in Publikationen, welche Bezug auf die Identifikation der Parameter nehmen (z.b. Dargazany und Itskov (2009)), oft nur wenige Angaben darüber, wie sich das Stoffgesetz im Identifikationsprozess verhält. Gleiches gilt auch für die Robustheit der Simulationen bei ungünstigen Parameterkonstellationen, welche während der Identifikation entstehen können. Die Auswahlkriterien lassen sich demnach wie folgt zusammenfassen:

- Abbildung von Hysterese, Restdeformation und Mullins-Effekt

- Stabilität des Stoffgesetzes bei starker Deformation der finiten Elemente

- Robustes Konvergenzverhalten auch bei ungünstigen Parameterkonstellationen

- Verfügbarkeit in $M S C . M A R C$ sowie $A B A Q U S$ bzw. Aufwand der Implementierung in eine Nutzerschnittstelle

Die Entscheidung fiel zugunsten des von Ihlemann (2003) entwickelten Model of Rubber Phenomenology (MORPH) in Kombination mit dem Konzept repräsentativer Raumrichtungen (Ihlemann, 2007). Für das Stoffgesetz lag zu diesem Zeitpunkt bereits eine Implementierung für die beiden verwendeten FEM-Programme in Form einer entsprechenden Nutzerschnittstelle vor und es war möglich, auf Erfahrungen bezüglich der Anpassung an experimentelle Daten (Hohl, 2007) zurück zu greifen. 


\subsubsection{MORPH in repräsentativen Richtungen}

Das MORPH-Stoffgesetz (Model of Rubber Phenomenology) wurde in erster Linie zur Darstellung der nichtlinearen, inelastischen Effekte von gefüllten Gummiwerkstoffen entwickelt. Mit nur 8 Parametern sowie dem Kompressionsmodul ist die Abbildung typischer Eigenschaften wie Hystereseerscheinungen, Entfestigung und verbleibender Restverformung auch bei großen Verzerrungen möglich. Im Folgenden werden die wesentlichen Teile des Materialmodells in kompakter Form dargestellt, wobei die ausführliche Herleitung Ihlemann (2003) entnommen werden kann.

Entsprechend der LAGRANGEschen Formulierung setzen sich die Gesamtspannungen (2. Piola-Kirchhoff-Spannungstensor $\underline{\underline{T}}$ ) des Stoffgesetzes aus folgenden Teilen zusammen:

$$
\underline{\underline{\widetilde{T}}}=2 \alpha \underline{\underline{C}}^{\prime} \cdot \underline{\underline{C}}^{-1}+\left(\underline{\underline{\widetilde{T}}}^{Z} \cdot \underline{\underline{C}}\right)^{\prime} \cdot \underline{\underline{C}}^{-1}-p J_{3} \underline{\underline{C}}^{-1}
$$

Der erste Teil beschreibt die Grundspannungen, welche für konstante $\alpha$ (was im Allgemeinen nicht der Fall ist) der NEO-HoOKE-Formulierung entsprechen. Die Variable $\alpha$ ist in Gl. (6.10) definiert und beinhaltet verschiedene Materialparameter $p_{i}$ sowie den Schleppzeiger $C_{\mathrm{T}}^{\mathrm{S}}$, welcher das Maximum der TRESCA-Invariante von $\stackrel{G}{\underline{G}}$ während der gesamten Belastungsgeschichte darstellt:

$$
\begin{gathered}
C_{\mathrm{T}}^{\mathrm{S}}(t)=\max \left[\stackrel{G}{C}_{\mathrm{T}}(\tau) ; 0 \leq \tau \leq t\right] \\
\operatorname{mit}: \stackrel{G}{C}_{\mathrm{T}}=\max \left[\left|G_{I}^{G}-\stackrel{G}{C}_{J}\right| ; I, J=1,2,3\right]
\end{gathered}
$$

Das bedeutet, dass nach Erreichen der maximalen Verzerrung die Grundspannungen ein rein hyperelastisches Materialverhalten beschreiben. Im zweiten Teil ist die Entwicklung von Zusatzspannungen $\underline{\underline{T}}^{Z}$ durch eine Differentialgleichung definiert:

$$
\underline{\underline{\underline{T}}}^{Z}=\beta \stackrel{G}{L}_{\mathrm{T}}\left(\underline{\underline{\widetilde{T}}}^{H}-\underline{\underline{T}}^{Z}\right)+\frac{1}{3}\left(\underline{\underline{T}}^{Z} \cdot . \underline{\underline{C}}\right) \underline{\underline{\underline{C}}}^{-1}-\frac{1}{3}\left(\underline{\underline{C}}^{-1} \cdot . \stackrel{\underline{\underline{C}}}{\underline{\underline{T}}} \underline{\underline{T}}^{Z} \quad \text { mit } \quad \underline{\underline{T}}_{0}^{Z}=0 .\right.
$$

Hierbei werden die begrenzenden Hüllspannungen $\underline{\underline{T}}^{H}$ mittels einer Exponentialfunktion berechnet:

$$
\stackrel{\underline{\widetilde{T}}}{ }^{H}=\left(\gamma \exp \left(\left(p_{7} \frac{\underline{\underline{\underline{L}}}}{\bar{L}_{\mathrm{T}}} \frac{\stackrel{G}{C_{\mathrm{T}}}}{C_{\mathrm{T}}^{\mathrm{S}}}\right)\right)+p_{8} \frac{\underline{\underline{\underline{L}}}}{L_{\mathrm{T}}}\right) \cdot \underline{\underline{C}}^{-1} .
$$

Der Tensor $\stackrel{G}{\underline{G}}$ kann als LAGRAnGEsches Äquivalent zur ZAREMBA-JAUmAnN-Zeitableitung des isochoren Links-CAUCHY-GREEn-Tensor interpretiert werden. Die entsprechende TRESCA-Invariante wird mit $\stackrel{G}{L}$ T ausgedrückt:

$$
\stackrel{G}{\underline{L}}=\frac{1}{2}\left(\underline{\underline{C}}^{-1} \cdot \underline{\underline{C}}+\underline{\underline{C}} \cdot \underline{\underline{C}}^{-1}\right)^{\prime} \cdot \underline{\underline{C}} .
$$


Die Größen $\alpha, \beta$ und $\gamma$ werden wir folgt dargestellt:

$$
\begin{aligned}
& \alpha=p_{1}+p_{2} f\left(p_{3} C_{\mathrm{T}}^{\mathrm{S}}\right) \\
& \beta=p_{4} f\left(p_{3} C_{\mathrm{T}}^{\mathrm{S}}\right) \\
& \gamma=p_{5} C_{\mathrm{T}}^{\mathrm{S}}\left(1-f\left(C_{\mathrm{T}}^{\mathrm{S}} / p_{6}\right)\right)
\end{aligned}
$$

mit

$$
f(x)=\frac{1}{\sqrt{1+x^{2}}}
$$

Darüber hinaus wurde die uniaxiale Form des tensoriellen Stoffgesetzes in das Konzept der repräsentativen Richtungen (RepRicht) (Ihlemann, 2007; Freund und Ihlemann, 2010) integriert. Für die Verwendung dieses Prinzips werden anhand eines vorgegebenen dreidimensionalen Verzerrungszustandes die Streckungen gedachter materialfester Linien in diskreten, möglichst gleichmäßig verteilten Richtungen bestimmt. Für jede dieser "repräsentativen Richtungen" lässt sich mit der uniaxialen Materialbeschreibung eine einachsige Spannungsantwort angeben. Der Gesamtheit aller Richtungen wird anschließend über die Forderung gleicher Formänderungsleistung durch numerische Integration über die Kugeloberfläche ein vollständiger Spannungstensor zugeordnet. Dieser wird wiederum als Antwort auf die vorgegebene Deformation interpretiert. Durch die Anwendung des Konzeptes auf das MoRPH-Stoffgesetz kann günstigerweise automatisch eine weitere Eigenschaft technischer Gummiwerkstoffe, die belastungsinduzierte Anisotropie, abgebildet werden. Zudem verhält sich das Stoffgesetz in FEM-Simulationen stabiler, sodass auch komplizierte Bauteilsimulationen konvergieren.

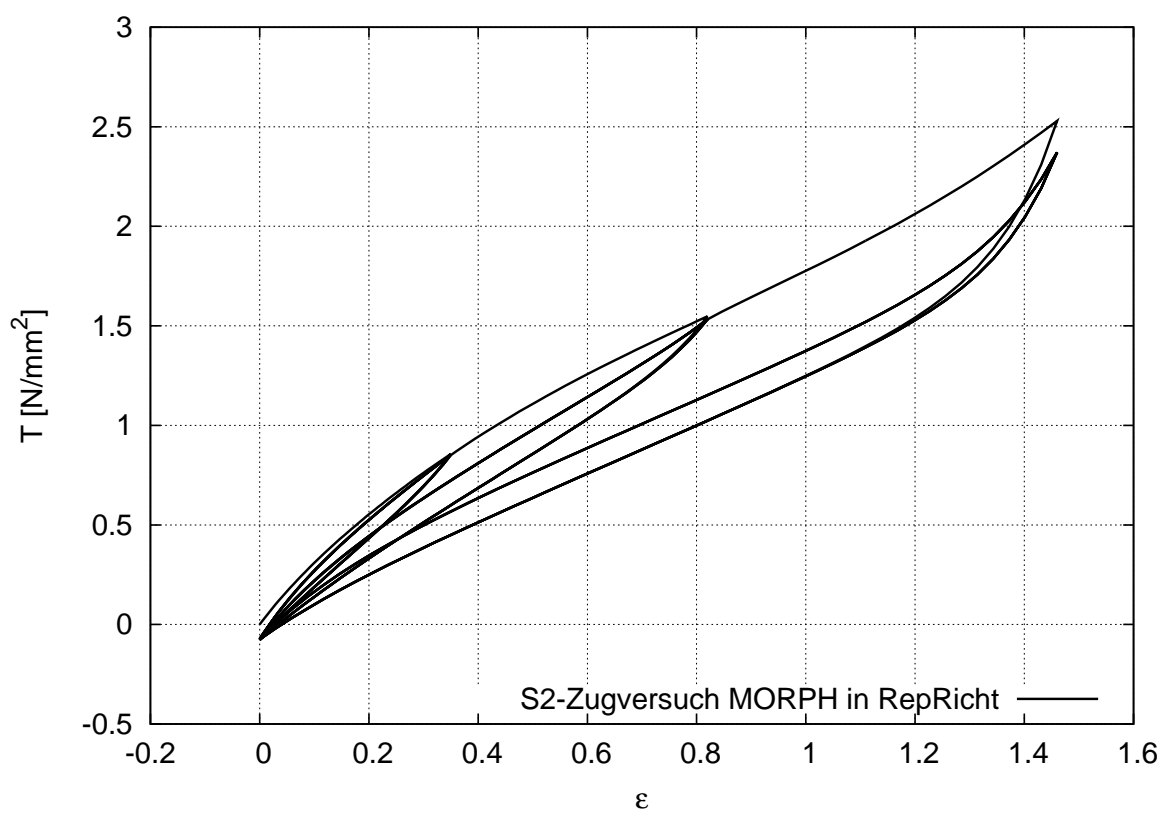

Bild 6.11: Spannungs-Dehnungs-Verlauf für einen mehrstufigen einachsigen Zugversuch mit jeweils 5 Zyklen bei Nutzung von MORPH in RepRicht.

In Bild 6.11 ist beispielhaft der Verlauf eines mehrstufigen Zugversuches mit dem in Tabelle 6.12 angegebenen Startparametersatz zur Beschreibung eines gefüllten Elastomers dargestellt. Die Belastung erfolgt verschiebungsgesteuert und sowohl der Hysterese-Effekt als auch die Entfestigung und die verbleibende Restdeformation sind gut sichtbar. Aufgrund 
der Abhängigkeit der Größe der Entfestigung bzw. der Restdeformation vom Schleppzeiger verhält sich jeder Belastungszyklus ab der zweiten Wiederholung quasistationär. Dies ist im Hinblick auf die Identifikation sehr vorteilhaft, da in den Experimenten zwar fünf oder mehr Wiederholungen jeder Lastamplitude zum Erreichen der Gleichgewichtshysterese benötigt werden, diese aber direkt dem quasistationären Zustand der entsprechenden Lastamplitude in der Simulation nach der zweiten Wiederholung zugeordnet werden können. Der notwendige Rechenaufwand kann somit deutlich gesenkt werden.

Die zum Zeitpunkt des Projektstarts "Identifikation anhand bauteilnaher Probekörper" vorliegende Implementierung des Stoffgesetzes in den Nutzerschnittstellen von MSC.MARC und $A B A Q U S$ war für Anwendungen im wissenschaftlichen Bereich konzipiert worden. Bezüglich einer nutzerfreundlichen Verwendung im industriellen Umfeld sowie einer effizienten Nutzung innerhalb von Parameteridentifikationen mussten deshalb noch einige Modifikationen vorgenommen werden. So existierte je eine separate Implementierung für MSC.MARC und ABAQUS, weswegen sämtliche Änderungen oder Erweiterungen des Materialmodells in zwei voneinander abweichenden Nutzerschnittstellen vorgenommen werden mussten. Durch Einführung gemeinsamer Namenskonventionen und Datenstrukturen konnten alle für das Stoffgesetz relevanten Routinen zusammengeführt werden, sodass für jedes FEM-Programm nur noch eine einzelne ummantelnde Funktion notwendig ist. Die Schnittstelle wurde zudem derart erweitert, dass bei Einhaltung einfacher Namenskonventionen während der Modellerstellung auch mehrere unterschiedliche Materialbereiche definiert werden können, welche sich sowohl bezüglich des gewählten Materialmodells als auch der zugehörigen Parametersätze unterscheiden können. Weitere umfangreiche Änderungen betrafen die Art der Verwaltung und Variation der Materialparameter im Rahmen der Identifikation sowie die Ausgabesteuerung der für die Identifikation wichtigen Simulationsergebnisse (Kap. 2.4).

\subsubsection{Verwendung von Stabilisierungselementen}

Im Gegensatz zu üblichen FEM-Simulationen im wissenschaftlichen Bereich müssen in der Industrie oft Belastungen an komplexen Bauteilgeometrien, welche zudem oft zu sehr großen Deformationen führen, durch FEM-Simulationen dargestellt werden. Bereits bei der Simulation der Belastungen des Bauteils unter Verwendung des robusten YEOH-Stoffgesetzes traten teilweise Konvergenzschwierigkeiten auf (Kap. 6.2). Der Grund dafür ist, dass infolge der Bauteilgeometrie und verstärkt durch den vorhandenen Selbstkontakt einige Elemente dazu tendieren, aufgrund der großen lokalen Verzerrungen zu entarten. Nähern sich einzelne Innenwinkel der Grenze von $180^{\circ}$, ist ein Abbruch der Simulation sehr wahrscheinlich. Aufgrund der Komplexität des inelastischen Stoffgesetzes sind derartige Innenwinkel umso kritischer. Da eine gezielte Vernetzung zur Vermeidung dieser Effekte nicht für alle Belastungsarten erfolgreich war, wurde ein anderer Ansatz zur Stabilisierung der Simulation entwickelt. Zu diesem Zweck werden in jeder Ecke von Elementen, welche potentiell entarten können, zusätzliche sogenannte "Stabilisierungselemente " eingebaut. Ein solches Stabilisierungselement wird erst dann aktiv, wenn der zugehörige Winkel sich einer vom Nutzer gewählten kritischen Grenze (z.B. $180^{\circ}$ ) nähert und reagiert auf die drohende Entartung mit einer zunehmenden Versteifung der Ecke. Ein Überschreiten der kritischen Grenze ist nicht 
möglich und die Stabilität der Simulation bleibt somit gewahrt. Die Stabilisierung erfolgt, indem für jeden Gausspunkt der Stabilisierungselemente ein Wichtungsfaktor $q$ auf Basis der Volumenänderung $J_{3}$ berechnet wird:

$$
q=\alpha \cdot \beta \cdot\left(\ln J_{3}\right)^{\beta-1} \quad \text { mit } \quad J_{3}=\sqrt{I_{3}(\underline{\underline{C}})} .
$$

Hierbei wird das Ansprechverhalten des Wichtungsfaktors auf die Volumenänderung durch die Steuerparameter $\alpha$ und $\beta$ gesteuert. In Bild 6.12 sind die Verläufe des Wichtungsfaktors für verschiedene Kombinationen der Steuerparameter beispielhaft dargestellt. Davon ausgehend wird die zugehörige Spannung $\underline{\underline{T}}$ ermittelt, welche für große $q$ zu einer zunehmenden Versteifung im Gausspunkt führt:

$$
\underline{\underline{T}}=q \cdot \underline{\underline{C}}^{-1}
$$

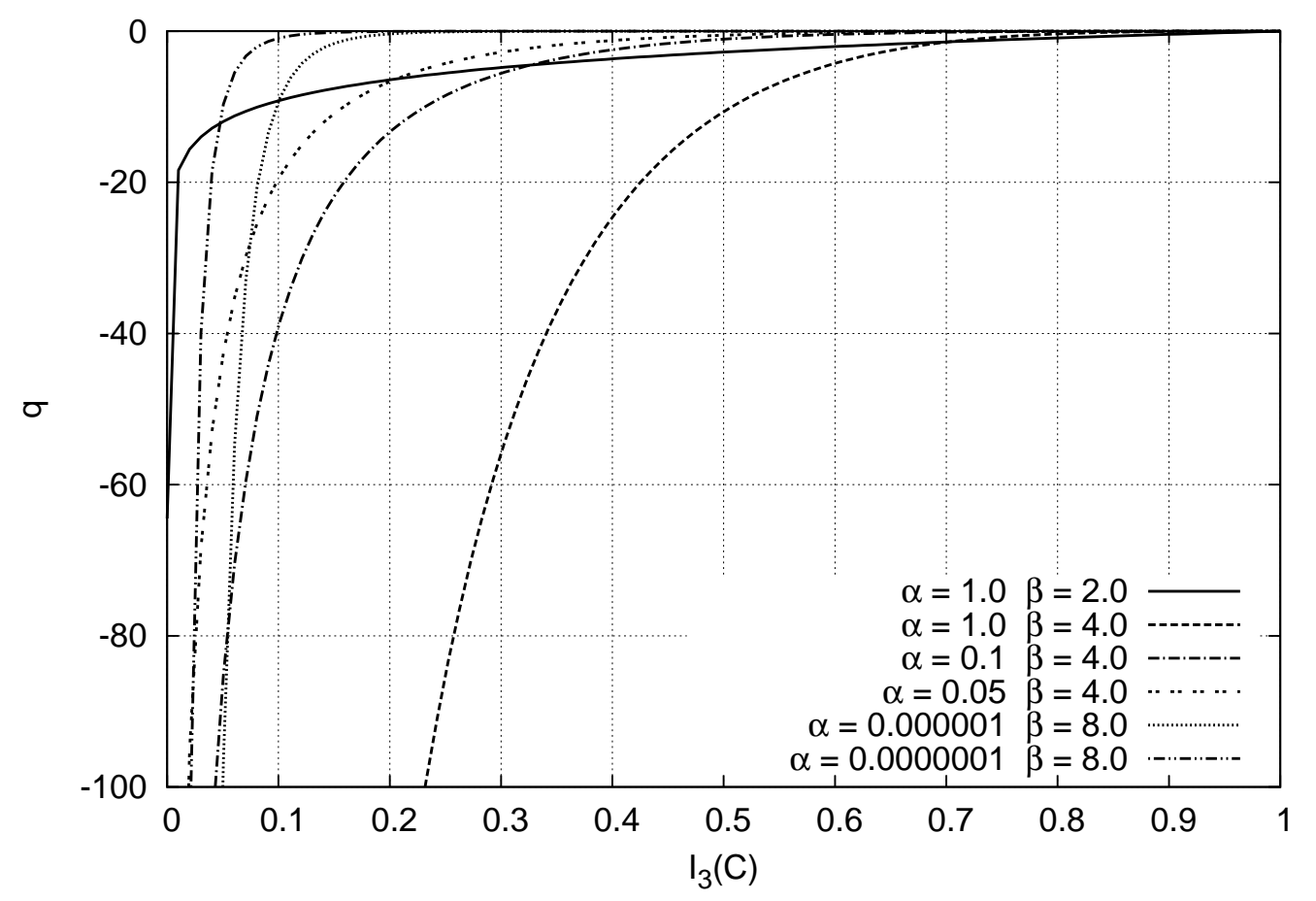

Bild 6.12: Verlauf des Wichtungsfaktors $q$ in Abhängigkeit der 3. Hauptinvariante des Rechts-CAUCHY-GreEn-Tensors.

Zwar verfälscht eine solche lokale Versteifung die Abbildungsgenauigkeit des Bauteilverhaltens, jedoch hat dies auf die globalen, integralen Größen nur einen geringen Einfluss. Dies belegt beispielhaft der Vergleich der axialen Belastung des bauteilnahen Probekörpers mit und ohne Stabilisierung in Bild 6.13. Bis zum Zeitpunkt des Abbruches der Simulation aufgrund entarteter Elemente verhalten sich beide FE-Modelle praktisch identisch. Diese Aussage ist nur insoweit einzuschränken, dass kein beträchtlicher Teil des FE-Gebietes von Entartung betroffen sein sollte und die daraus folgende Versteifung großer Bereiche auch die integralen Größen maßgeblich beeinflusst. 


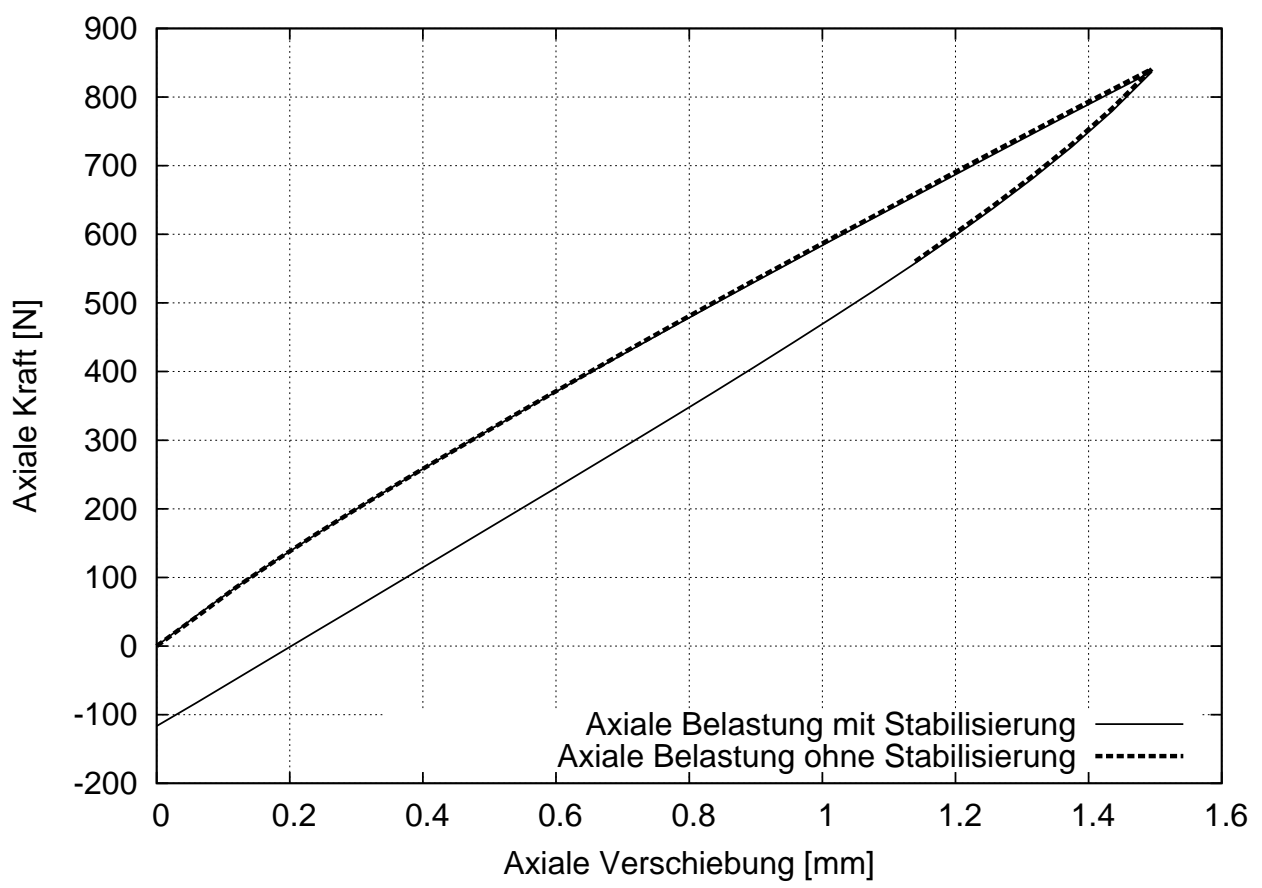

Bild 6.13: Simulationsvergleich der axialen Belastung eines bauteilnahen Probekörpers ohne und mit Verwendung von Stabilisierungselementen.

Die Verwendung des MORPH-Stoffgesetzes in repräsentativen Richtungen in Kombination mit der Nutzung zusätzlicher Stabilisierungselemente in FE-Gebieten mit potentiellen Konvergenzschwierigkeiten erweist sich durchweg als vorteilhaft. Selbst anspruchsvolle Simulationen am Bauteil wie die kardanische Belastung der Fahrzeugbuchse konvergieren erfolgreich, obwohl dies bei Verzicht auf die Stabilisierungselemente und Nutzung des robusten YEOH-Ansatzes aufgrund entartender Elemente nicht der Fall ist.

\subsection{Bauteilsimulation}

Zur Darstellung der verschiedenen Belastungen aus Kap.6.2 in diesbezüglichen FEM-Simulationen ist zunächst eine Vernetzung des Bauteils notwendig. Im Rahmen des Forschungsvorhabens wurden dafür, ebenso wie für die anschließenden Simulationen, die kommerziellen FEM-Programme MSC.MARC* und $A B A Q U S^{*}$ genutzt. Aufgrund der Tatsache, dass die prinzipielle Vorgehensweise unabhängig vom gewählten FEM-Programm ist, wird im Folgenden nur auf die mittels MSC.MARC durchgeführten Simulationen Bezug genommen.

Da lediglich das Verhalten der Gummischicht von Interesse ist, wird auch nur diese als FE-Modell benötigt - die Berücksichtigung der Außen- und Innenhülse erfolgt durch Definition entsprechender Randbedingungen. Die Vernetzung der Gummischicht des realen Bauteils ist in Bild 6.14 dargestellt, wobei Abweichungen von der Rotationssymmetrie vernachlässigt wurden.

${ }^{*}$ Detaillierte Angaben sind im Anhang $[$ A.1 aufgeführt. 


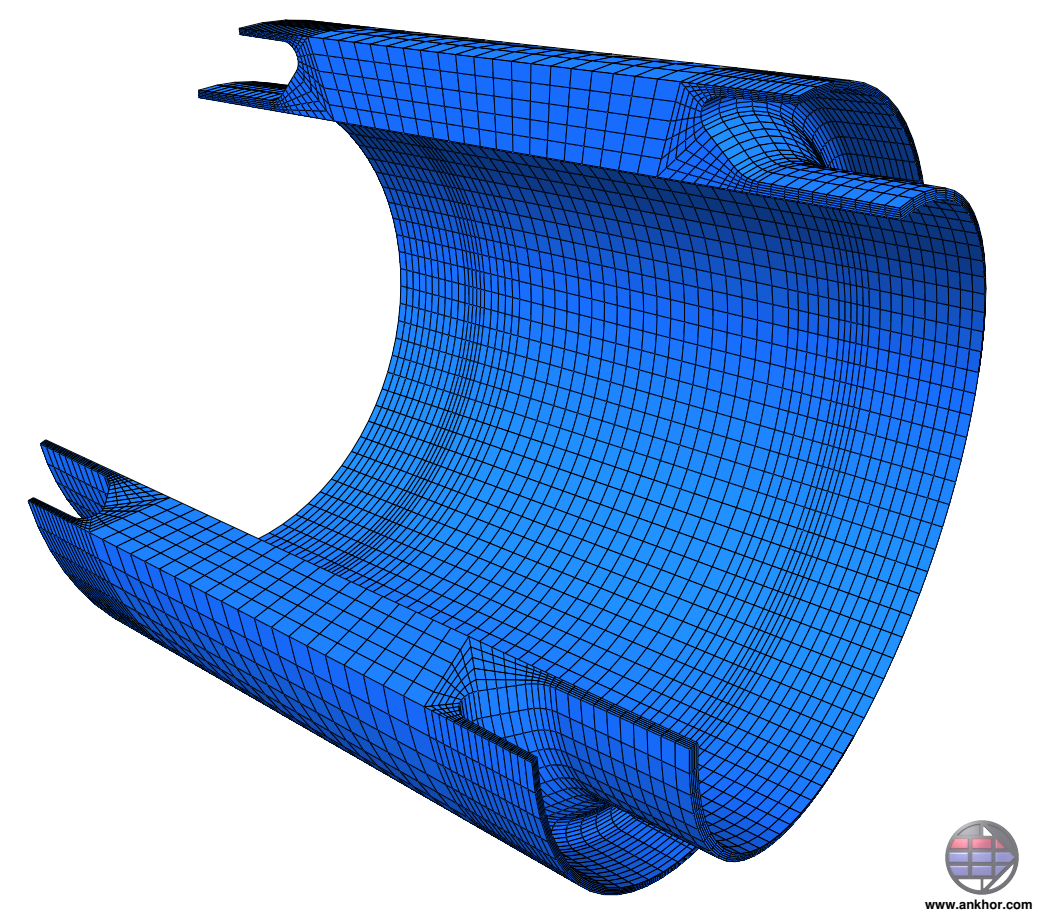

Bild 6.14: FE-Modell des Originalbauteils der Anvis-Buchse.

Zwar ist die Geometrie der Gummischicht nicht übermäßig kompliziert, jedoch stellen die durch Kalibrierung und Belastung bedingten großen Verzerrungen in einigen Bereichen des Modells sowie der entstehende Selbstkontakt eine große Herausforderung dar. Durch die Nutzung der in Kap. 6.4.2 beschriebenen Stabilisierungselemente können dennoch alle Beanspruchungen des Lastenhefts simuliert werden.

Zur Darstellung der Simulationsergebnisse bietet sich ein geometrisches Vergleichsmaß an, da vor allem die erheblichen Verzerrungen für Elastomere im Allgemeinen und für die hier betrachteten Lastfälle im Besonderen typisch und somit zur Bewertung besonders hilfreich sind. Zudem ist die Aussagekraft von Spannungsgrößen, gerade in Gebieten mit potentieller Entartung, nur begrenzt zuverlässig (siehe Kap. 6.4.2). Gewählt wird deshalb, analog zur Berechnung der plastischen Vergleichsverzerrung (z.B. nach Gross (1992)), die auf Basis des

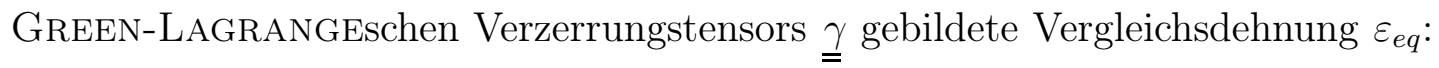

$$
\varepsilon_{e q}=\sqrt{\frac{2}{3} \underline{\underline{\gamma}} \cdot \underline{\underline{\gamma}}} \quad \text { mit } \quad \underline{\gamma}=\frac{1}{2}\left(\underline{\underline{F}}^{T} \underline{\underline{F}}-\underline{\underline{I}}\right)
$$

Die Simulationen, welche den nachfolgenden Darstellungen zugrunde liegen, wurden unter Verwendung von MORPH in RepRicht mit den in Tabelle 6.18 angegeben Parameter durchgeführt. Zusätzlich sind in den FE-Modellen (in den Abbildungen nicht sichtbare) Stabilisierungselemente enthalten, um das Auftreten entarteter finite Elemente, speziell im Bereich des Selbstkontaktes, zu vermeiden. Bevor die Bauteilbeanspruchungen aufgebracht werden, müssen vorher auch in der Simulation der Abkühlprozess sowie die Kalibrierung der Buchse berücksichtigt werden. In Bild 6.15 ist das deformierte Bauteil nach dem Abkühlen um $150 K$ abgebildet. Der anschließende Kalibriervorgang führt zu dem in Bild 6.16 dargestellten 
Zustand. Obwohl die verschiedenen Belastungen noch nicht aufgebracht wurden, sind einige Bereiche des Bauteils bereits stark deformiert.

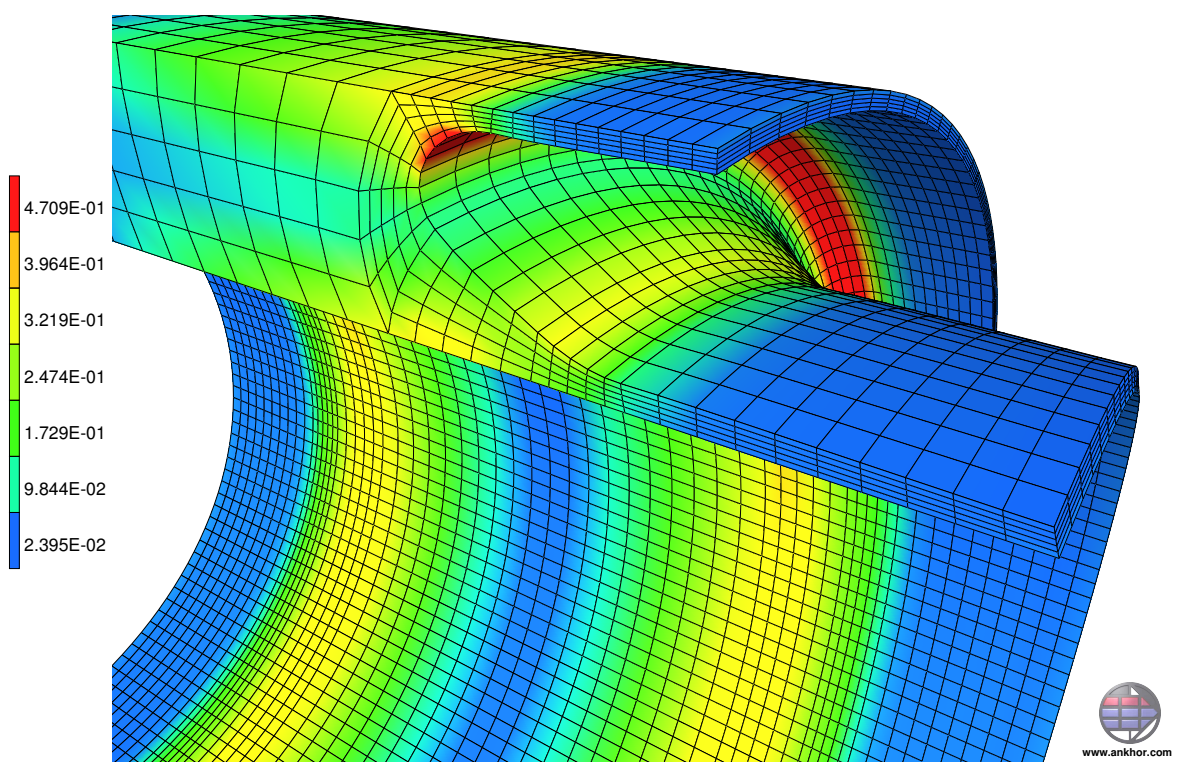

Bild 6.15: Vergleichsdehnungen im Bauteil nach dem Abkühlen.

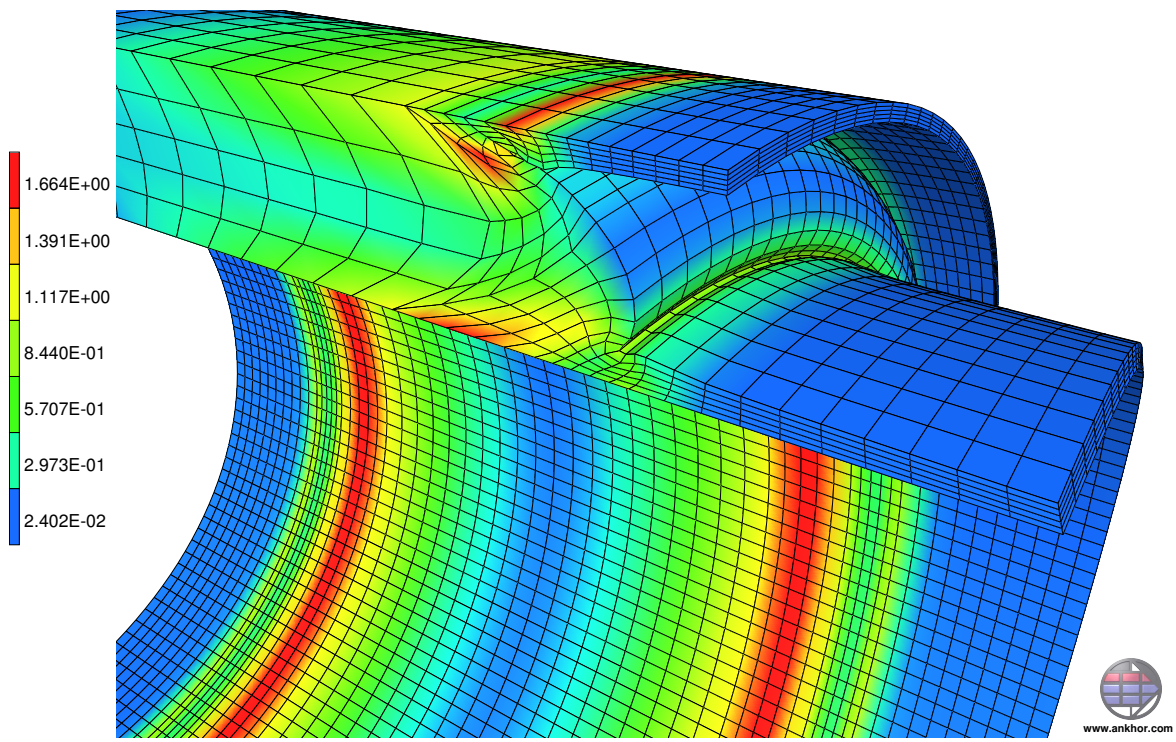

Bild 6.16: Vergleichsdehnungen im Bauteil nach dem Kalibrieren.

Die verschiedenen Beanspruchungen werden jeweils nach Abkühlung und Kalibrierung der Buchse simuliert. Um den quasistationären Zustand zu erreichen, werden die Belastungszyklen zwei Mal in Folge mit der gleichen Amplitude aufgebracht.

Bei der Simulation der axialen Bauteilbelastung kann aufgrund des axialsymmetrischen Charakters ein rotationssymmetrisches FE-Modell genutzt werden. Die wiederholte axiale Belastung des Bauteils mit $F^{\text {axial }}=1 \mathrm{KN}$ führt auf die in Bild 6.17 dargestellten Vergleichsdehnungen. 


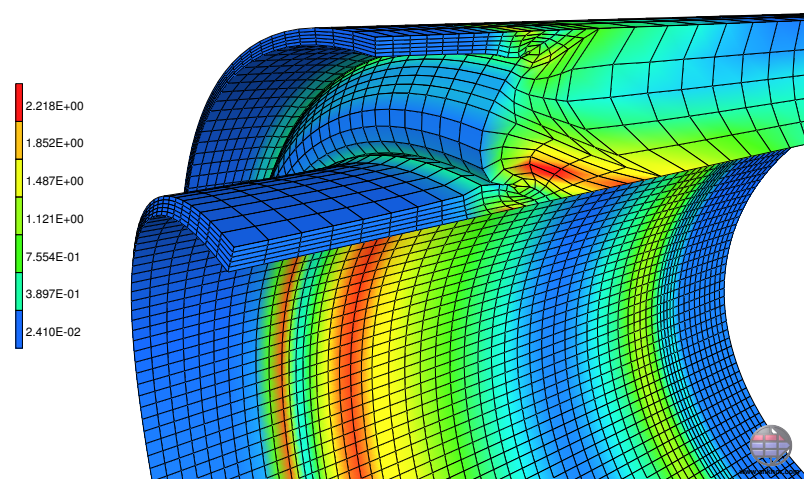

(a) Rückseite

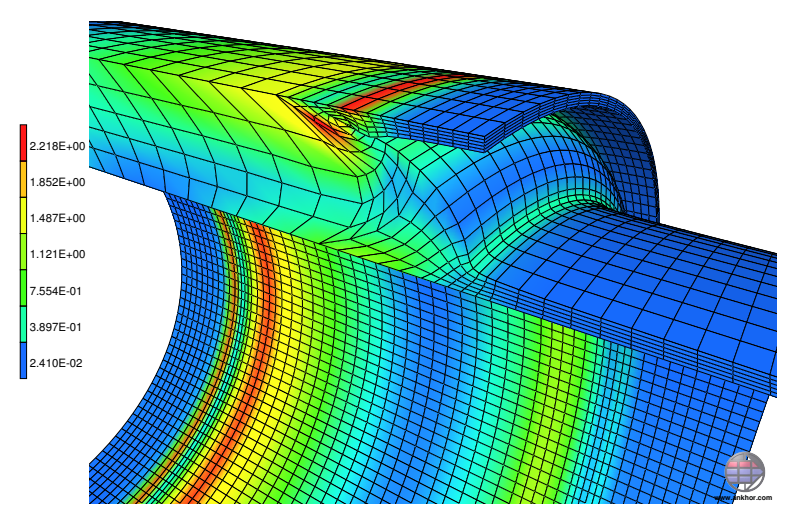

(b) Vorderseite

Bild 6.17: Vergleichsdehnungen bei axialer Belastung (1 KN).

Da die radiale Belastung nicht rotationssymmetisch ist, muss ein 3D-Netz für die FEMSimulation verwendet werden. Allerdings kann die Größe des Modells durch Ausnutzung der Symmetrieebenen in axialer Richtung sowie der Symmetrieebene, welche den mittigen Meridianschnitt darstellt, geviertelt werden. Die Simulation für die radiale Maximalbelastung von $F^{r a d}=10 \mathrm{KN}$ führt auf die in Bild 6.18 dargestellten Vergleichsdehnungen. Dabei treten in einigen Elementen sehr ungünstige Innenwinkel auf, was zu einer entsprechenden Versteifung der dort befindlichen Stabilisierungselemente führt. Dies führt zwar lokal zu Fehlern, hat auf die globalen Reaktionsgrößen jedoch keinen signifikanten Einfluss.

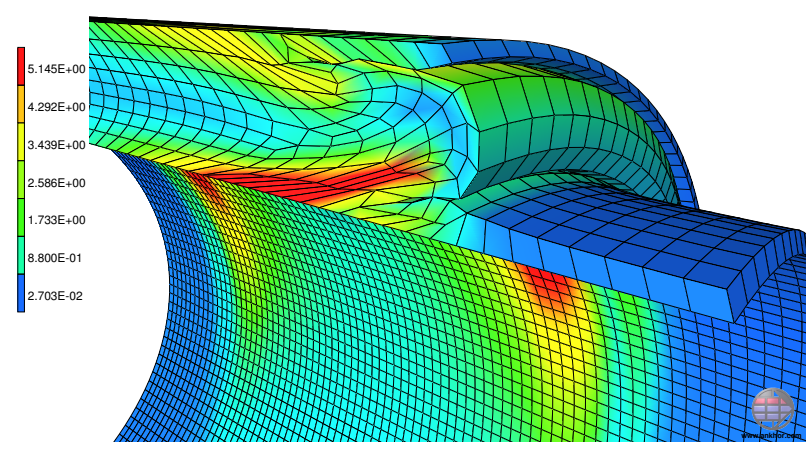

(a) Oberseite

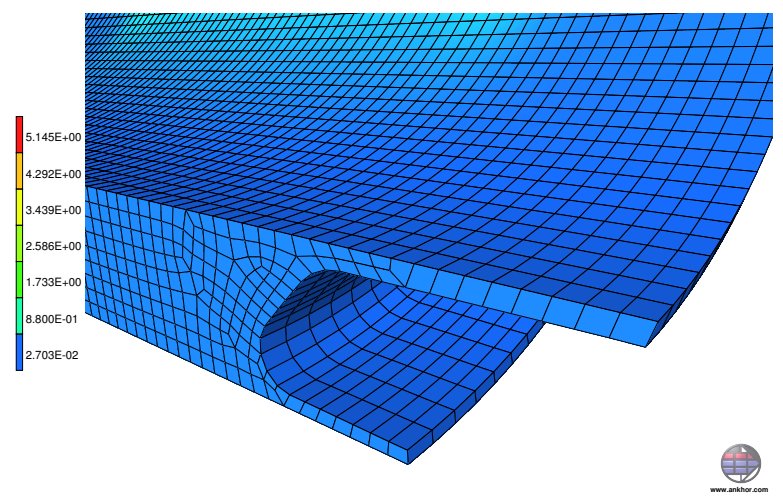

(b) Unterseite

Bild 6.18: Vergleichsdehnungen bei radialer Belastung (10 KN).

Auch bei der kardanischen Beanspruchung handelt es sich nicht um eine rotationssymmetrische Belastung, womit ebenfalls ein 3D-Netz für die FEM-Simulation notwendig ist. Zur Reduktion der Größe des Modells kann lediglich die Symmetrieebene, welche den mittigen Meridianschnitt darstellt, genutzt werden. Die Simulation für die kardanische Maximalverdrehung von $\varphi^{\text {kard }}=3^{\circ}$ führt auf die in Bild 6.19 dargestellten Vergleichsdehnungen. Analog zur radialen treten auch bei der kardanischen Belastung in einigen Bereichen durch Stabilisierungselemente induzierte Spannungen auf, welche das Ergebnis lokal beeinflussen. 


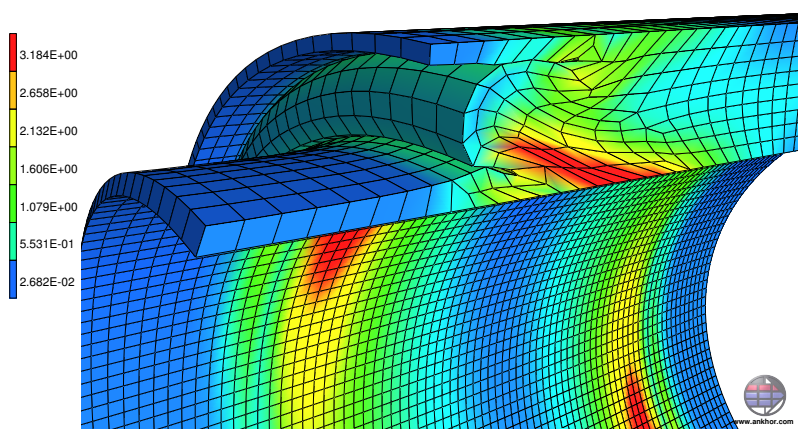

(a) Vorderseite

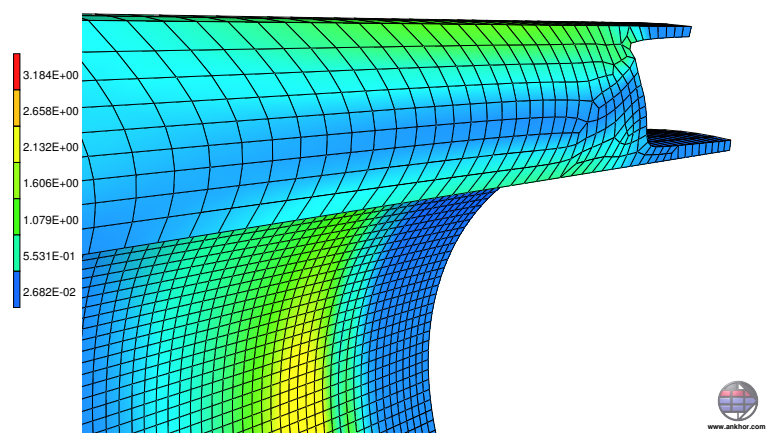

(b) Rückseite

Bild 6.19: Vergleichsdehnungen bei kardanischer Belastung $\left(3^{\circ}\right)$.

Für die Simulation von Torsionsbelastungen steht im FEM-Programm MSC.MARC ein spezieller Elementtyp (Twist \& Herrmann Formulation) zur Verfügung. Bei Verwendung dieses Typs würde ein rotationssymmetrisches FE-Modell für die Berechnung genügen. Leider beschränkt sich die Darstellungsgenauigkeit der entsprechenden Simulationen nur auf kleine Verdrehungen, weshalb eine Nutzung an dieser Stelle nicht möglich ist. Um dennoch kein 3D-Modell nutzen zu müssen, wird der Meridianschnitt der Gummischicht durch Extrusion in Umfangsrichtung in ein dünnes Schichtmodell umgewandelt und mit zyklischen Randbedingungen versehen. Der Extrusionswinkel beträgt 1,25 und stellt einen Kompromiss zwischen gutem Konvergenzverhalten (je kleiner der Extrusionswinkel, desto schlechter) und hoher Abbildungsgenauigkeit (je größer der Extrusionswinkel, desto ungenauer) dar. Die Simulation für die Maximalverdrehung von $\varphi^{\text {Tors }}=15^{\circ}$ führt auf die in Bild 6.20 dargestellten Vergleichsdehnungen, wobei zur Verdeutlichung der starken Verwölbung des Meridianschnitts nochmals der kalibrierte Zustand zum Vergleich aus dem gleichen Blickwinkel zu sehen ist.

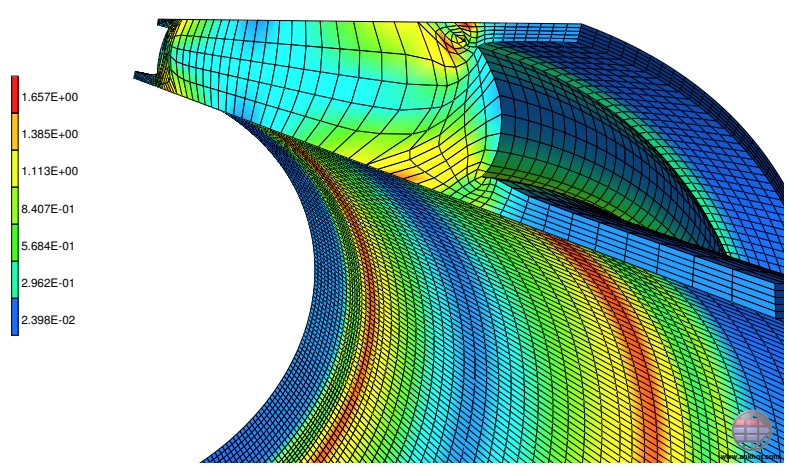

(a) Nach Kalibrierung

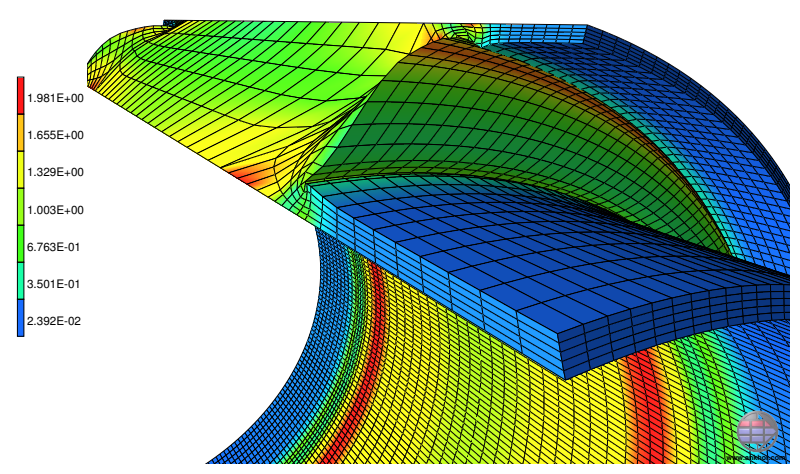

(b) Nach Torsionsbeanspruchung

Bild 6.20: Vergleichsdehnungen bei Torsionsbeanspruchung $\left(15^{\circ}\right)$.

Aufgrund der großen Elementzahl in den 3D-Modellen der radialen und der kardanischen Belastung sind diese FEM-Simulationen sehr rechenintensiv. Hinzu kommt, dass die bereits durch Kalibrierung entstandenen Gebiete mit Selbstkontakt durch die Belastungen nochmals größer werden und das Konvergenzverhalten deutlich beeinflussen. Nur durch eine geschickte 
Kontaktformulierung können hier unerwünschte Effekte wie die Durchdringung gegenüber liegender Flächen oder das "Hängenbleiben" einzelner Knoten vermieden werden.

Die Materialparameter, welche in den dargestellten Simulationen verwendet wurden, sind zu Beginn eines Projektes gewöhnlich nicht bekannt. Zwar ist es legitim, einen bereits bekannten Parametersatz des Materialmodells von einer vergleichbaren Gummimischung zu nutzen, um vorab eine grobe Bewertung der Simulationen vornehmen zu können. Jedoch ist angesichts der Tatsache, dass neben der gewählten Gummimischung sowohl die Geometrie als auch der Fertigungsprozess des Bauteils die Materialparameter maßgeblich beeinflussen, die Identifikation der Parameter des Stoffgesetzes absolut notwendig.

\subsection{Identifikation mit Standardprobekörpern}

Die Identifikation von Materialparametern wird üblicher Weise mittels standardisierter Laborprüfkörper durchgeführt. Diese sind gewöhnlich durch eine sehr einfache Geometrie gekennzeichnet und die Belastungen sind derart gewählt, dass sie als näherungsweise homogen charakterisiert werden können. Für die Nutzung solcher Standardprobekörper sprechen mehrere Fakten:

- Die notwendige Rechenkapazität zur Ermittlung der für die Identifikation notwendigen Zielfunktionswerte ist außerordentlich gering.

- Konvergenzschwierigkeiten spielen unabhängig vom gewählten Stoffgesetz praktisch keine Rolle, da häufig die Betrachtung eines einzelnen Materialpunktes mit einem aufgebrachten Belastungszustand genügt.

- Das Materialverhalten wird durch die Beschränkung auf homogene Belastungen unmittelbar charakterisiert.

- Standardprobekörper können preisgünstig und in großer Zahl hergestellt werden. Da sich deren Geometrie nicht an den Bauteilen orientiert, können sowohl die Herstellungswerkzeuge als auch die Prüfvorrichtungen für jede Identifikation erneut verwendet werden.

Vor allem der letzte Punkt stellt für industrielle Anwendungen einen großen Vorteil dar, da bei der Entwicklung neuer Bauteile oder Gummimischungen im ersten Schritt die exakte Abbildung des Materialverhaltens oft gar nicht im Vordergrund steht. Vielmehr genügt meist erst einmal eine grobe Abschätzung, welche dafür aber möglichst schnell zur Verfügung stehen muss. Unter anderem deshalb ist die Identifikation anhand von Standardprobekörpern in der Industrie sehr gebräuchlich und stellt auch hier die Grundlage weiterer Betrachtungen dar. Sowohl die Herstellung der Standardprobekörper als auch die Durchführung der entsprechenden Versuche erfolgten am DIK Hannover. 


\subsubsection{Auswahl der Probekörpergeometrien}

Nach wie vor werden in vielen Bereichen der Industrie Stoffgesetzanpassungen noch ausschließlich anhand der Messdaten aus einachsigen Zugversuchen (S2-Zugversuch) durchgeführt. Die eigentliche Anpassung, meist durch hyperelastische Stoffgesetze, stellt dabei gewöhnlich kein Problem dar. Dies ist beispielhaft für das OGDEN-Stoffgesetz mit zwei Potenztermen in Bild 6.21 dargestellt. Hierbei, wie auch im Folgenden, stellt $\varepsilon$ die technische Dehnung und $T$ die 1. Piola-KIrChHOFF-Spannung (technische Spannung) dar.

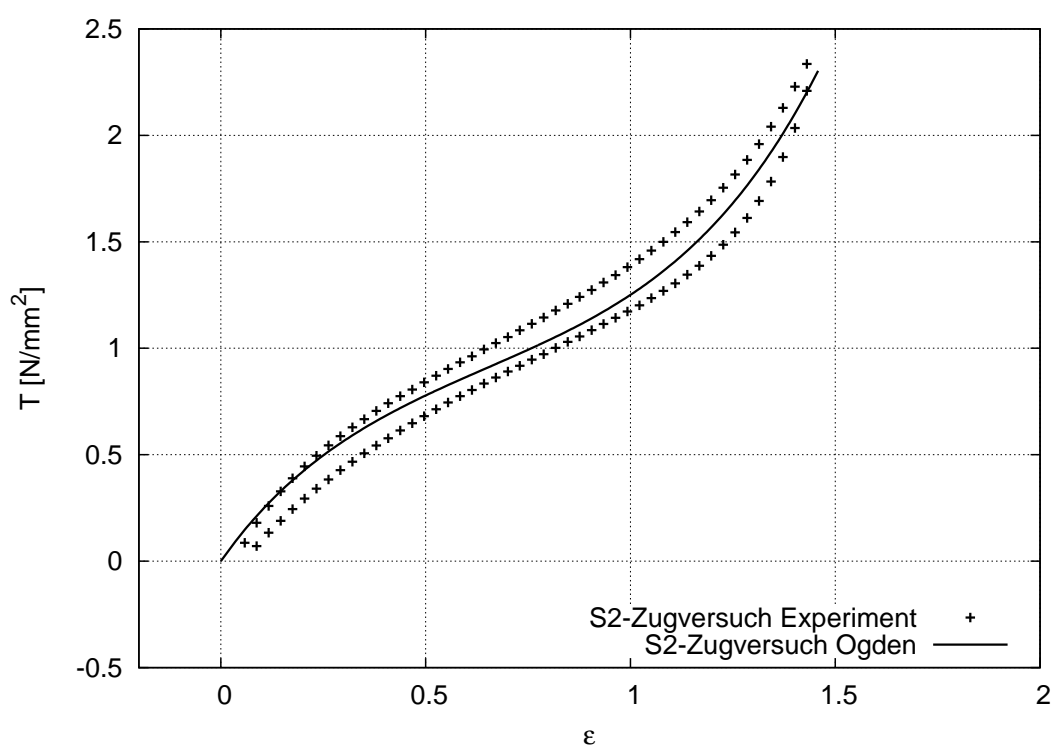

Bild 6.21: Spannungs-Dehnungs-Diagramm auf Basis des S2-Zugversuches Kurvenverlauf für identifizierte OGDEN-Parameter $(n=2)$.

Die Anwendung auf die Simulation anderer homogener Belastungsarten (Bild 6.22) zeigt jedoch eine deutliche Abweichung gegenüber den zugehörigen Messungen.

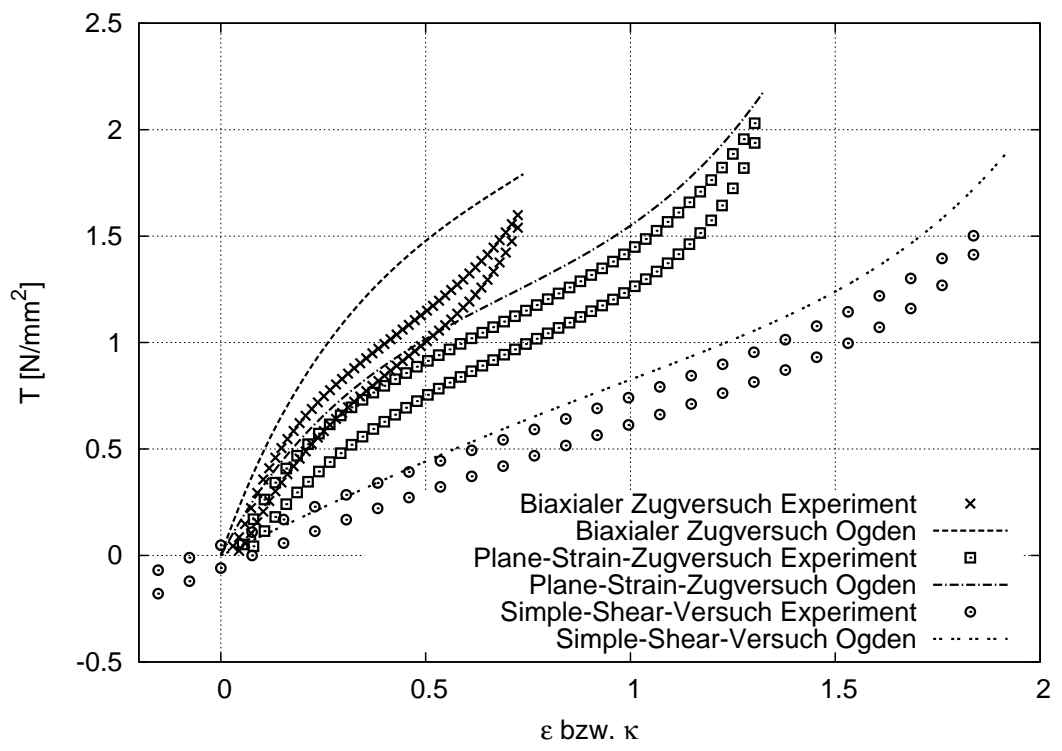

Bild 6.22: Spannungs-Dehnungs-Diagramm auf Basis des S2-Zugversuches Kurvenverläufe für identifizierte OGDEN-Parameter $(n=2)$. 
Der Grund dafür liegt nicht nur in den beschränkten Abbildungsmöglichkeiten der elastischen Stoffgesetze, sondern darin, dass der S2-Zugversuch eine sehr spezielle Belastungsart darstellt. In Bild 6.23 sind die möglichen Beanspruchungen eines Materialpunktes bei angenommener Volumenkonstanz $\left(I_{3}=1\right)$ im $I_{1}-I_{2}$-Raum gekennzeichnet. Daraus wird ersichtlich, dass insbesondere der S2-Zugversuch nur den Rand des möglichen Belastungsgebietes darstellt.

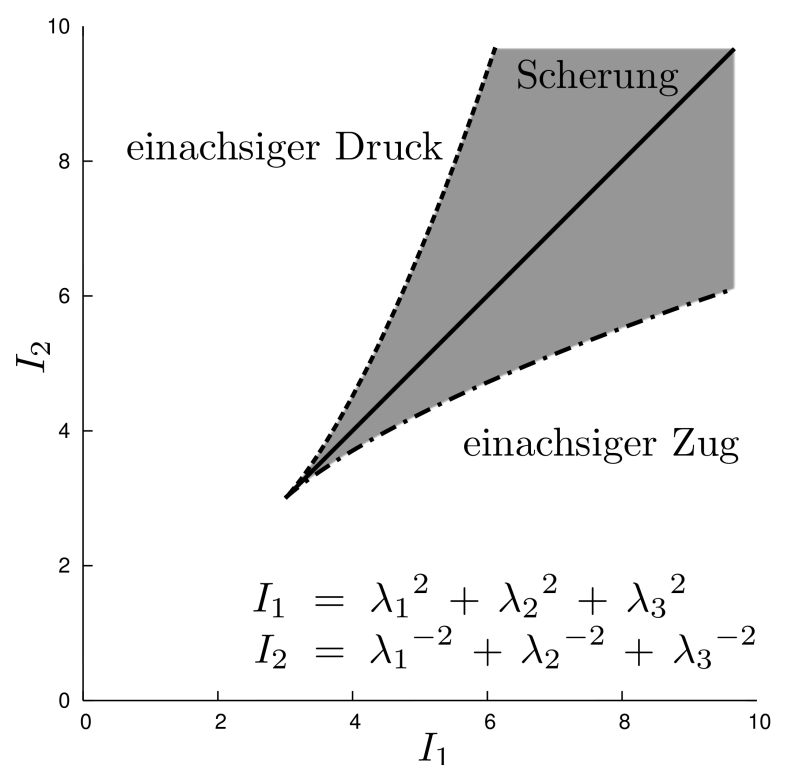

Bild 6.23: $I_{1}-I_{2}$-Diagramm für mögliche Kombinationen der Hauptstreckungen.

Um eine möglichst repräsentative Abbildung des gesamten Lösungsraumes zu erhalten, sollten möglichst viele unterschiedliche Belastungsarten in die Identifikation einbezogen werden. Im vorliegenden Projekt wurden deshalb vier verschiedene näherungsweise homogene Experimente durchgeführt:

- einachsiger Zugversuch an einem S2-Zugstab (Bild 6.24)

- biaxaler Zugversuch (Bild 6.25)

- Plane-Strain-Zugversuch (Bild 6.26)

- Simple-Shear-Versuch (Bild 6.27)

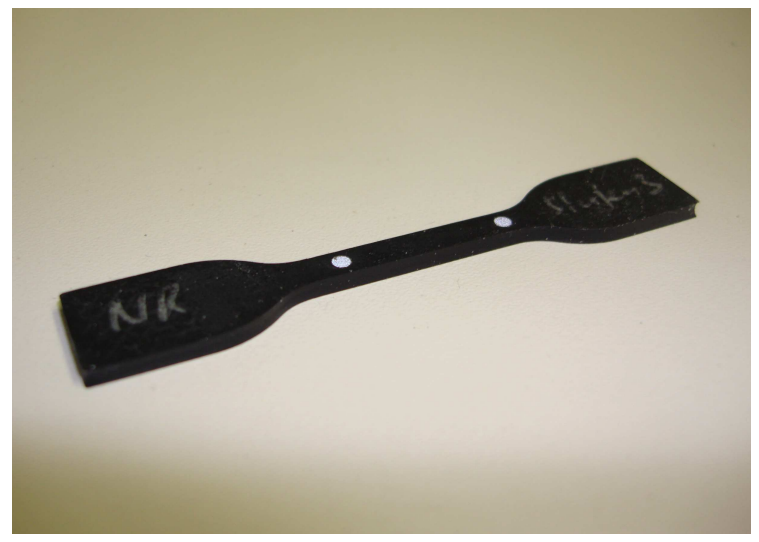

Bild 6.24: S2-Zugstab.

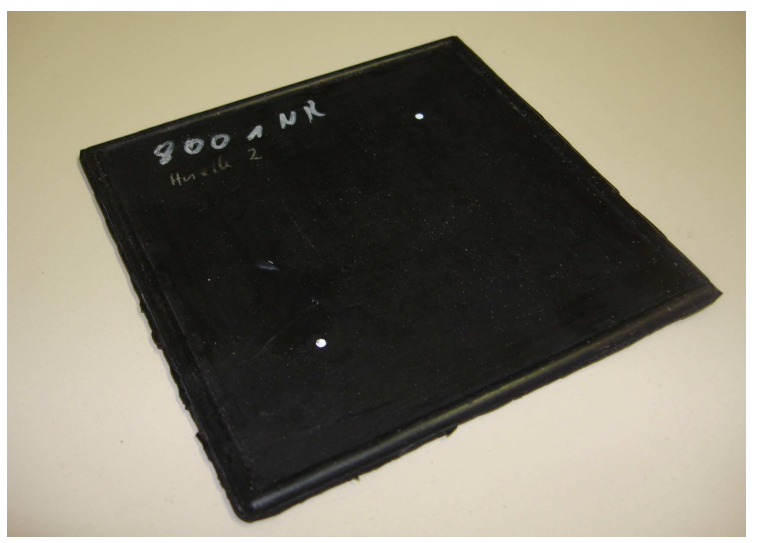

Bild 6.25: Biaxiale Zugprobe. 


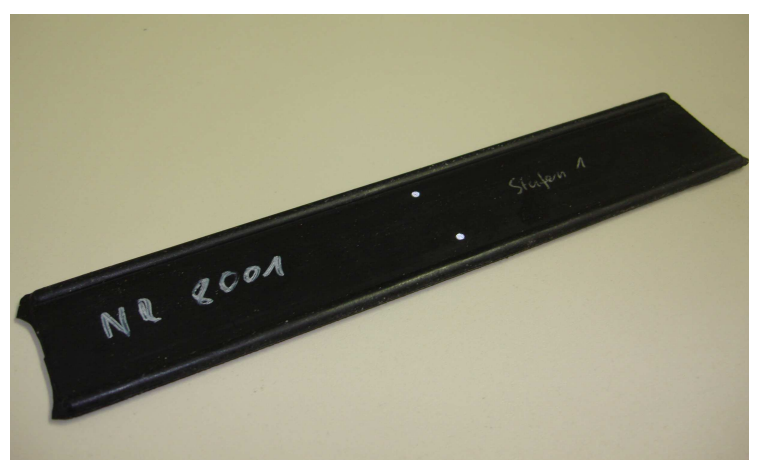

Bild 6.26: Plane-Strain-Probekörper.

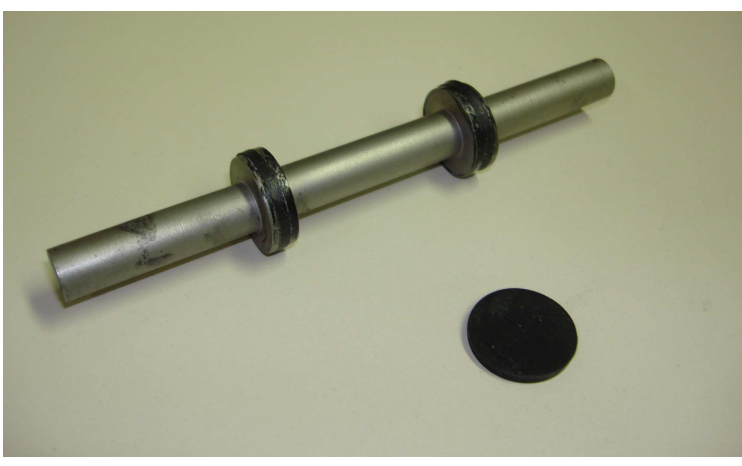

Bild 6.27: Simple-Shear-Probekörper.

Die Belastung erfolgte dabei jeweils weggesteuert, wobei in den Experimenten für jede Lastamplitude jeweils fünf Zyklen durchgeführt wurden. Um die Auswirkung von Messfehlern zu verringern, wurden die Experimente jeweils drei Mal wiederholt und die gewonnenen Messergebnisse anschließend gemittelt.

Da die Materialparameter nicht für den anfänglichen Zustand des Materials bestimmt werden sollen, werden die transienten Zyklen der Experimente nicht berücksichtigt. Lediglich der jeweils fünfte Zyklus, welcher bereits näherungsweise stationär ist,wird für die Identifikation verwendet. Dies ist am Beispiel des S2-Zugversuchs in Bild 6.28 dargestellt.

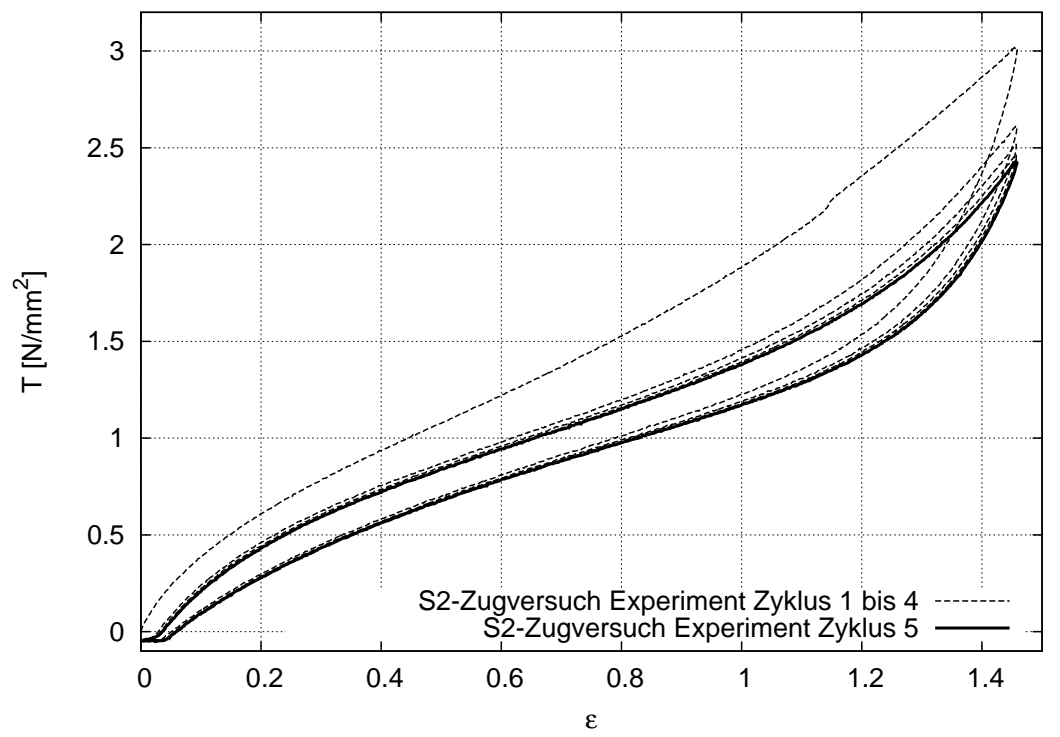

Bild 6.28: Spannungs-Dehnungs-Diagramm des S2-Zugversuches.

Darüber hinaus wurden auch mehrstufige Versuche durchgeführt. Die dabei gewonnen Ergebnisse werden benötigt, wenn statt eines hyperelastischen Stoffgesetzes ein komplexeres, inelastisches Stoffgesetz verwendet wird. Die Belastung wurde hierfür in drei Stufen unterteilt, wobei die Belastungszyklen jeder Stufe so oft wiederholt wurden, bis der jeweilige stationäre Zyklus näherungsweise erreicht wurde. Die Aufteilung der drei Stufen erfolgte linear bezüglich der dem S2-Zugversuch zugeordneten HENKY-Dehnung $h_{1}^{Z u g}$, was einer logarithmischen Aufteilung der entsprechenden Streckung $\lambda_{1}^{Z u g}$ entspricht und für den S2-Zugversuch in Bild 6.29 dargestellt ist:

$$
\lambda_{i}=e^{h_{i}} .
$$




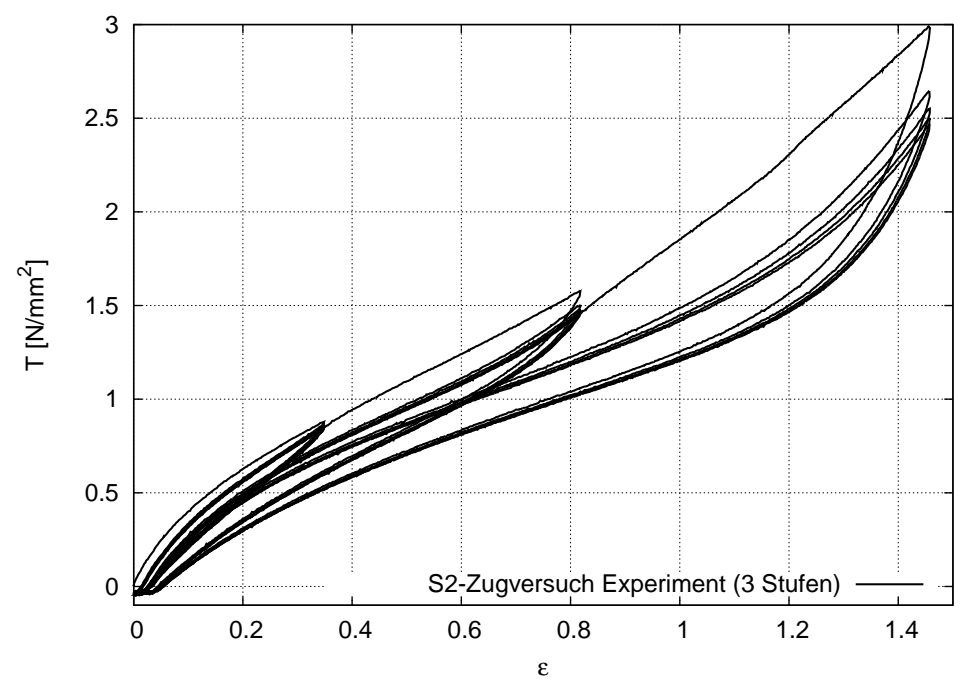

Bild 6.29: Spannungs-Dehnungs-Diagramm bezüglich des mehrstufigen S2-Zugversuches.

Die Durchführung der Versuche erfolgte ganz bewusst verschiebungsgesteuert. Dies bietet gegenüber der kraftgesteuerten Belastung den Vorteil, dass im Hinblick auf die Eigenschaft der belastungsinduzierten Entfestigung des verwendeten Materials schneller ein quasistationärer Zyklus erreicht wird. Viel wichtiger ist jedoch ein weiterer Aspekt. Bei der Verwendung von MORPH in RepRicht genügt für einen Belastungszyklus entsprechend Kap. 6.4.1 eine einzige Wiederholung zum Erreichen des quasistationären Zustands. Für einen dreistufigen Versuch genügt demnach die Darstellung von insgesamt sechs Belastungszyklen, um jedem quasistationären Zyklus aus dem Experiment einen entsprechend simulierten gegenüberstellen zu können. Gerade im Hinblick auf die rechenintensive Identifikation anhand bauteilnaher Probekörper ist dies außerordentlich wichtig. Der Nachteil dieses Vorgehens besteht darin, dass bei den Zugversuchen an den Standardprobekörpern am Ende der Rückverformung (Entlastungszyklen) infolge der Entfestigung bereits vor Erreichen des Ausgangspunktes die Spannungen Null werden und die nachfolgenden Messdaten bis zum erneuten Überschreiten dieser Grenze unbrauchbar, was z.B. Bild 6.28 deutlich zeigt. Eine mögliche Lösung bestünde darin, nur die Hinverformung weggesteuert durchzuführen und die Rückverformung kraftgesteuert. Dies war aus messtechnischer Sicht jedoch nicht möglich. Zudem ist es in einigen FEMProgrammen (z.B. bei MSC.MARC nur durch Nutzung der User-Subroutine "FORCDT") aufwändig, während eines Simulation zwischen den Belastungsarten zu wechseln - was im Hinblick auf die industrielle Anwendung nicht zu vernachlässigen ist. Im vorliegenden Projekt wurden die Zugversuche deshalb zwar vollständig verschiebungsgesteuert durchgeführt, die unbrauchbaren Messdaten bei der Anpassung jedoch nicht berücksichtigt.

Bei der Festlegung der Maximalbelastung für die Versuche muss berücksichtigt werden, dass die Zuverlässigkeit der identifizierten Materialparameter stark von der Abstimmung der homogenen Experimente auf die später typische Belastung in den FEM-Simulationen des Bauteils abhängig ist. In Bild 6.31 ist die Anpassung an das YEOH-Stoffgesetz bei unterschiedlichen Maximaldehnungen für den S2-Zugversuch dargestellt. Dabei wird deutlich, dass nahe 
dem Koordinatenursprung die Messdaten von der simulierten Kurve abweichen. Noch drastischer ist jedoch die Extrapolation des Materialverhaltens auf höhere Dehnungen - sie kann zu katastrophalen Ergebnissen führen. Es ist daher notwendig, die Höhe der Belastungen in den Experimenten auf die später typischen Belastungsintensitäten im Bauteil abzustimmen.

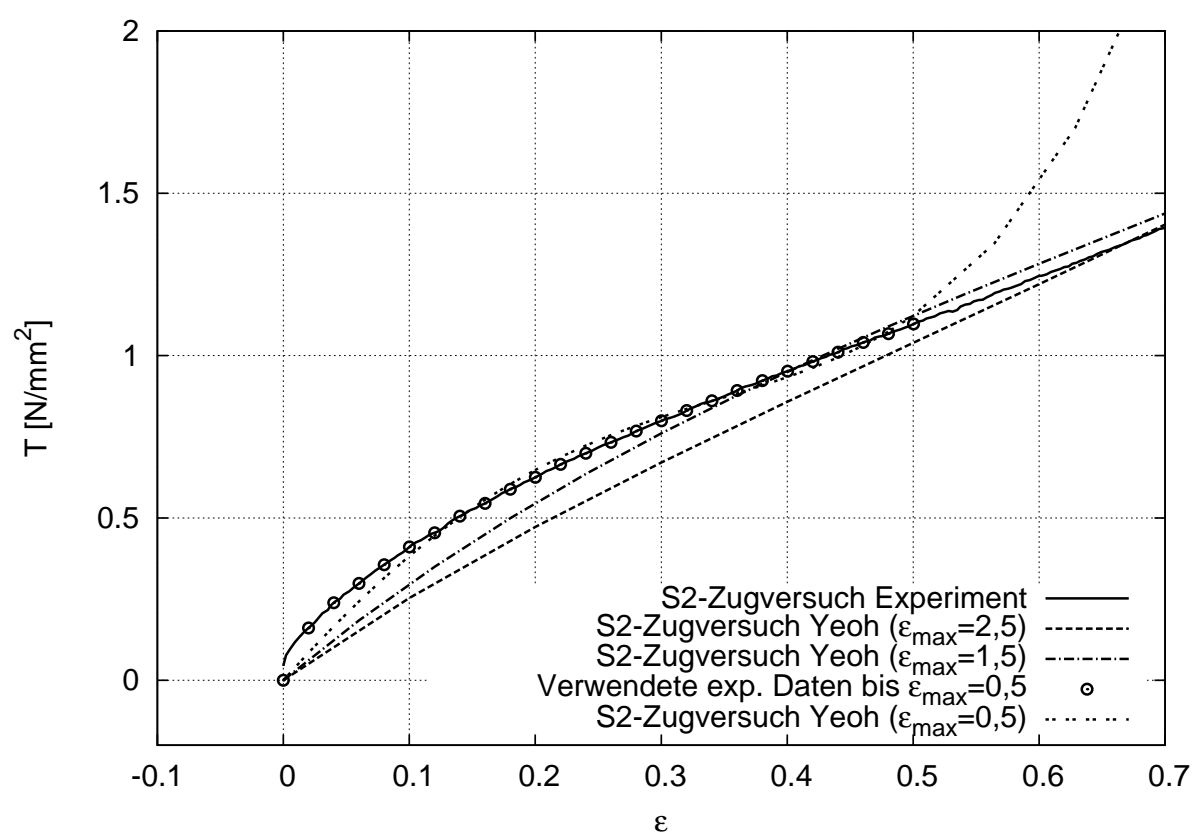

Bild 6.30: Spannungs-Dehnungs-Diagramm für den S2-Zugversuch bei Nutzung unterschiedlicher Maximaldehnungen.

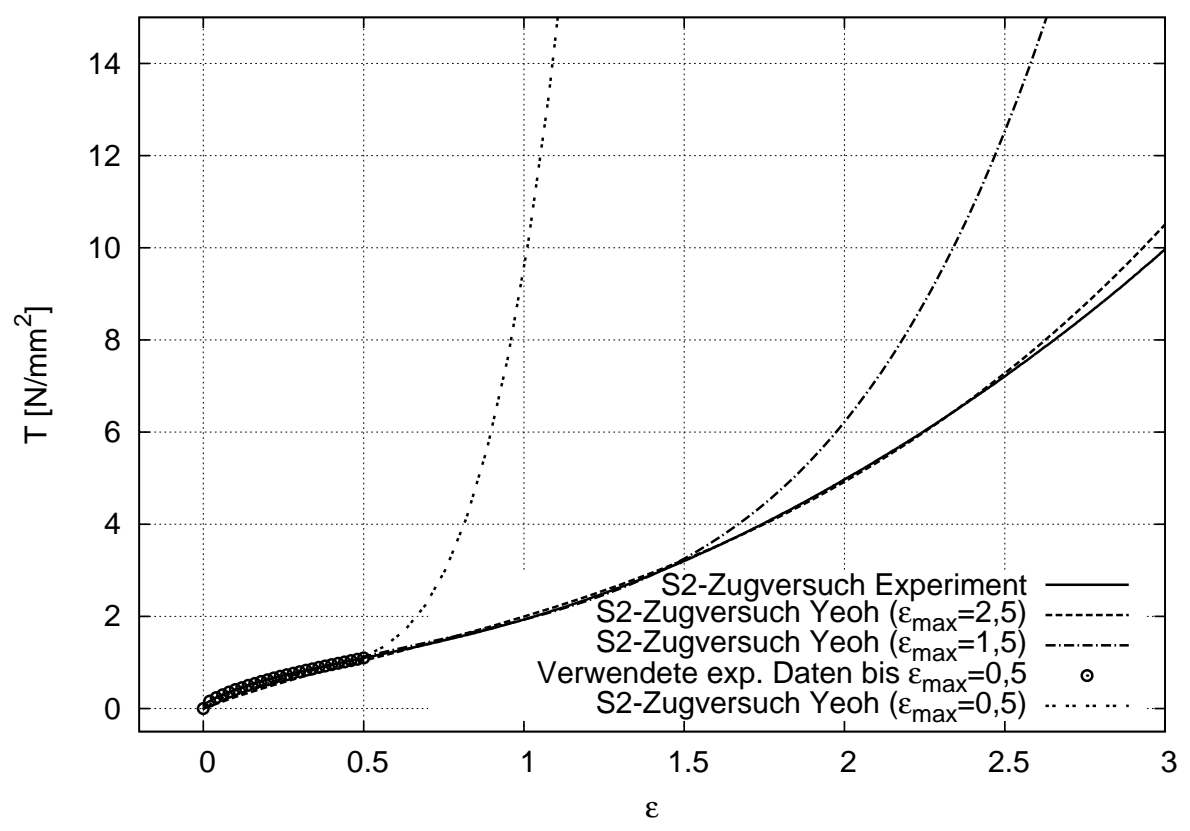

Bild 6.31: Spannungs-Dehnungs-Diagramm für den S2-Zugversuch bei Nutzung unterschiedlicher Maximaldehnungen. 


\subsubsection{Nullpunktverschiebung von Messdaten}

Im industriellen Umfeld werden entsprechend Kap. 6.4 vor allem hyperelastische Stoffgesetze genutzt. Diese sind jedoch nicht in der Lage, belastungsinduzierte Restverformungen darzustellen. Eben diese treten jedoch bei drei der vier hier betrachteten Versuche an Standardprobekörpern auf, da diese einsinnige Zugversuche darstellen. Das heißt, dass sich nach einem Belastungszyklus eine Restdehnung einstellt, welche bei mehrfacher Wiederholung der Lastamplitude einem quasistationären Wert entgegen strebt. Sollen nun Materialparameter identifiziert werden, stellt sich die Frage nach dem Umgang mit dieser Restdehnung. In der Dokumentation von $M S C . M A R C$ wird beispielsweise empfohlen, die erhaltenen Messkurven waagerecht in den Nullpunkt zu verschieben. Bei Rücksprachen mit den Projektpartnern wurde deutlich, dass dies auch in der Industrie gängige Praxis ist. Im Folgenden soll diese Vorgehensweise näher beleuchtet werden.

Hierfür werden die Hauptstreckungen der Versuche an den Standardprobekörpern (Bild 6.24 - Bild 6.27) benötigt, wobei $\lambda_{1}$ jeweils die Hauptstreckung in Belastungsrichtung kennzeichnet:

- S2-Zugversuch (Zug) mit $\lambda_{1}$ und $\lambda_{2}=\lambda_{3}=\frac{1}{\sqrt{\lambda_{1}}}$.

- Biaxial-Zugversuch (Biax) mit $\lambda_{1}, \lambda_{2}=\lambda_{1}$ und $\lambda_{3}=\frac{1}{\lambda_{1}^{2}}$.

- Plane-Strain-Zugversuch (Plane) mit $\lambda_{1}, \lambda_{2}=1$ und $\lambda_{3}=\frac{1}{\lambda_{1}}$.

- Simple-Shear-Versuch (Shear) mit dem Schermaß $\gamma$ bzw. $\kappa$, aus welchem sich die Hauptstreckungen ergeben.

$$
\begin{aligned}
& \lambda_{1}=\sqrt{1+\frac{\kappa^{2}}{4}}+\frac{\kappa}{2} \\
& \lambda_{2}=\sqrt{1+\frac{\kappa^{2}}{4}}-\frac{\kappa}{2} \\
& \lambda_{3}=1
\end{aligned}
$$

Für die prinzipielle Betrachtung der Nullpunktverschiebung soll der Anstieg eines an dieser Stelle abstrakten Versuches als linear angenommen werden. Die Zahlenwerte sind willkürlich gewählt und die Umrechnung der Messdaten soll beispielhaft für einen einachsigen Zugversuch betrachtet werden (Bild 6.32). 


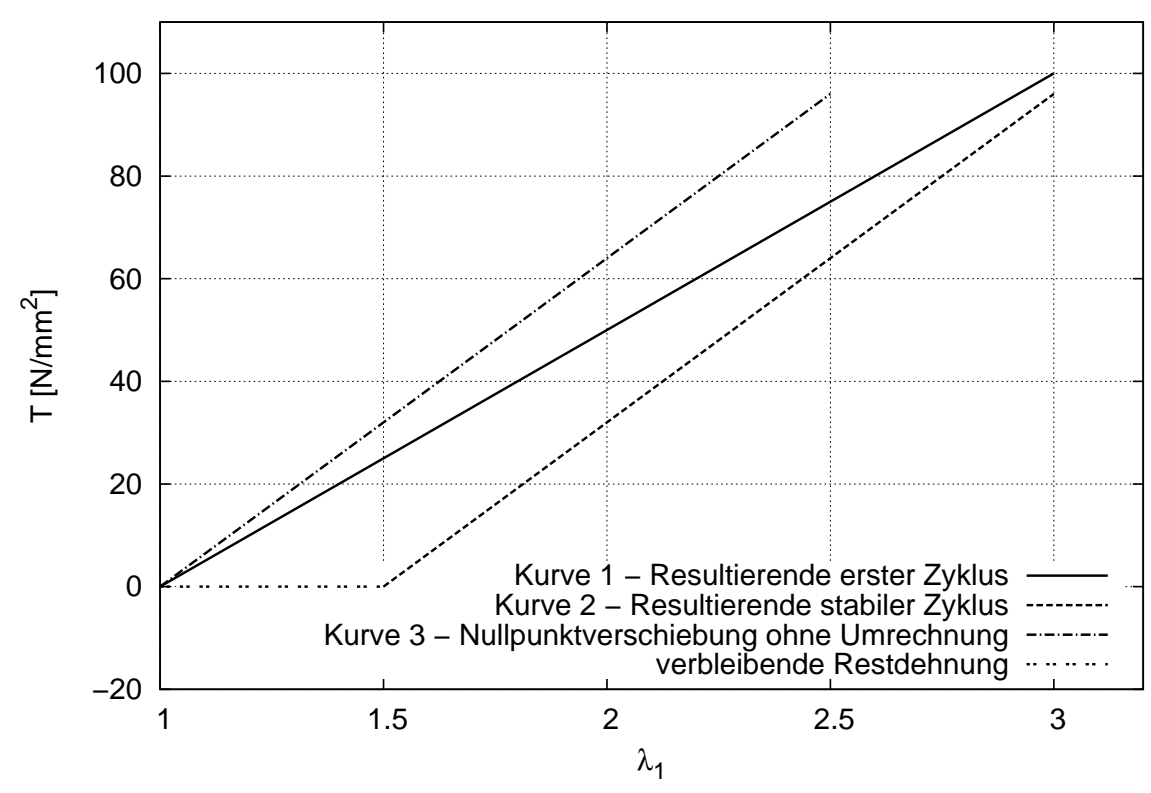

Bild 6.32: Nullpunktverschiebung der Messdaten ohne Umrechnung.

Die bei dem Versuch ermittelte 1. PiolA-KIRCHHOFF-Spannung $T$ kann mit der Ausgangsfläche $A_{0}$ sowie der gemessenen Kraft $F$ wie folgt ausgedrückt werden:

$$
T=\frac{F}{A_{0}} .
$$

Die zugehörige Streckung $\lambda$ ergibt sich aus der Ausgangslänge $l_{0}$ und der aktuellen Länge $l$ :

$$
\lambda=\frac{l}{l_{0}} .
$$

Der Fußpunkt von Kurve 2 liegt in Abhängigkeit der Restdehnung bei der Streckung $\lambda^{*}$ und ergibt sich zu

$$
\lambda^{*}=\frac{l^{*}}{l_{0}}>1
$$

Wird nun angenommen, dass eine Verschiebung von Kurve 2 in den Nullpunkt das gleiche Materialverhalten widerspiegelt, kann man dies mit einem weiteren Zugversuch prüfen. Praktisch bedeutet dies, dass Person A einen S2-Zugversuch 1 durchführt, die zugehörige Probe liegen lässt und eine Person B diese findet und ohne Vorkenntnis ihrerseits in Versuch 2 prüft. Um keine erneute Restdehnung zu erhalten, sei angenommen, dass die maximale gemessene Kraft in Versuch 2 der gemessenen Kraft im letzten Zyklus aus Versuch 1 entspricht. Somit ist die Messkurve bereits ab dem ersten Zyklus stationär.

Der Fußpunkt der Kurve liegt aus Sicht von Person B bei $\lambda_{\text {neu }}=1$, wobei sich die Streckung aufgrund der Vorbelastung auf $l^{*}$ bezieht:

$$
\lambda_{n e u}=\frac{l}{l^{*}} .
$$


Durch Erweiterung des Termes lässt sich der Bezug zu $\lambda$ aus dem 1. Versuch herstellen:

$$
\lambda_{\text {neu }}=\frac{l}{l^{*}}=\frac{l}{l_{0}} \frac{l_{0}}{l^{*}}=\lambda \frac{l_{0}}{l^{*}} .
$$

Die Spannung $T_{\text {neu }}$ bezieht sich nun auf die Querschnittsfläche $A^{*}$, welche aufgrund des nahezu inkompressiblen Materialverhalten durch die Gleichung

$$
l^{*} A^{*}=l_{0} A_{0}
$$

charakterisiert werden kann. Dies führt zu

$$
T_{\text {neu }}=\frac{F}{A^{*}}=\frac{F}{A_{0}} \frac{A_{0}}{A^{*}}=T \frac{A_{0}}{A^{*}}=T \frac{l^{*}}{l_{0}} .
$$

Betrachtet man den Anstieg der Messkurve aus Versuch 2, so wird diese steiler:

$$
\frac{\mathrm{d} T_{n e u}}{\mathrm{~d} \lambda_{\text {neu }}}=\frac{\mathrm{d} T}{\mathrm{~d} \lambda} \frac{l^{*} l^{*}}{l_{0} l_{0}}=\frac{\mathrm{d} T}{\mathrm{~d} \lambda}\left(\frac{l^{*}}{l_{0}}\right)^{2} .
$$

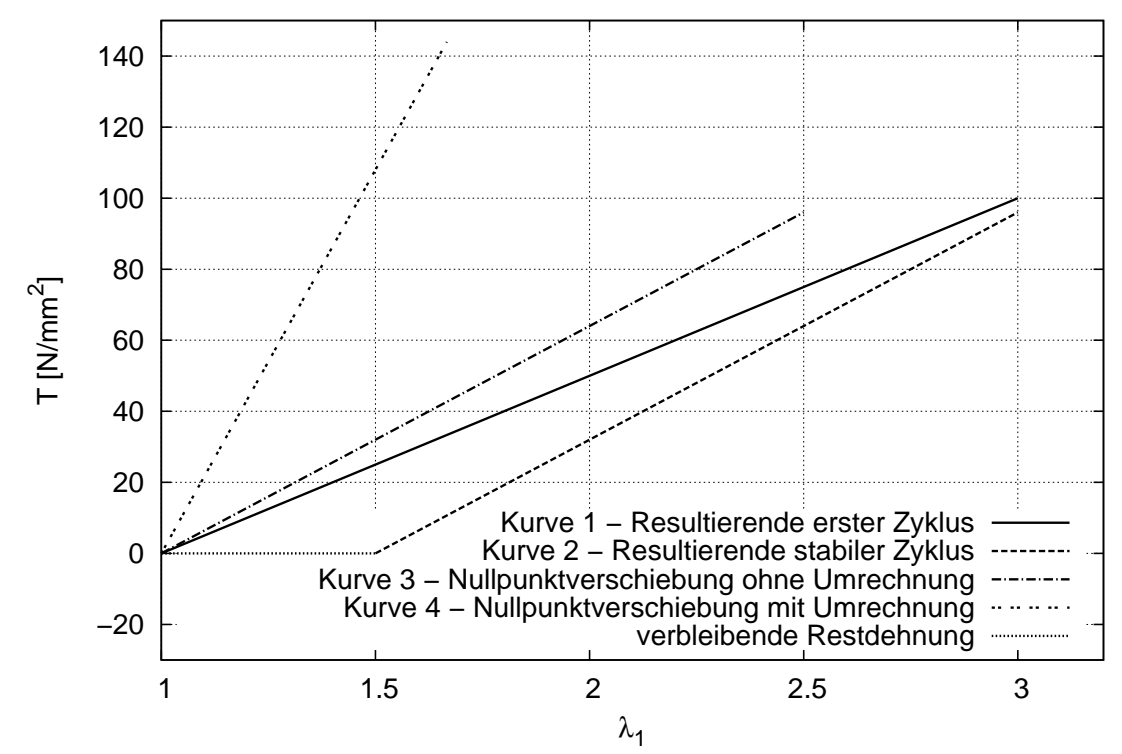

Bild 6.33: Nullpunktverschiebung der Messdaten mit Umrechnung.

Entsprechend Bild 6.33 bilden die Messkurve 2 aus Versuch 1 und die Messkurve 4 aus Versuch 2 zwar den gleichen Materialzustand ab, haben jedoch einen unterschiedlichen Anstieg. Eine bloße Nullpunktverschiebung ist demnach aus physikalischer Sicht nicht zulässig. Bei Umrechnung der Zugversuche entsprechend dem hier gezeigten Vorgehen muss beachtet werden, dass die anschließende Identifikation nicht für das unbelastete Material erfolgt. Vielmehr erhält man Parameter, welche das Verhalten des bereits vorbelasteten Werkstoffes beschreiben. Zudem gelten die Parameter nur dann, wenn die Belastungsintensität des Bauteils derjenigen im Versuch ähnelt, da andernfalls die der Umrechnung zugrunde liegende Restdehnung nicht mehr passen würde. 
Im Gegensatz zu den Zugversuchen genügt bei Simple-Shear-Versuchen eine bloße Verschiebung der Daten in den Nullpunkt. Die Schubspannung $\tau$ bezieht sich auch bei einer vorhandenen Restscherung $\kappa^{*}$ auf die gleiche Querschnittsfläche. Das zugeordnete Schermaß $\kappa$ ergibt sich aus

$$
\kappa=\frac{u}{d},
$$

wobei $d$ den Probendurchmesser und $u$ die Verschiebung in Querrichtung darstellen. Der Nulldurchgang $\kappa^{*}$ von Kurve 1, der Resultierenden des letzten Zyklus, ergibt sich aus

$$
\kappa^{*}=\frac{u^{*}}{d}
$$

Bei Messung der Kurve 2 gilt nun

$$
u_{\text {neu }}=u_{\text {alt }}-u^{*},
$$

welches auf Basis von

$$
\kappa_{\text {neu }}=\frac{u_{\text {neu }}}{d}
$$

$\mathrm{Zu}$

$$
\kappa_{\text {neu }}=\frac{u_{a l t}-u^{*}}{d}=\kappa-\kappa^{*}
$$

führt.

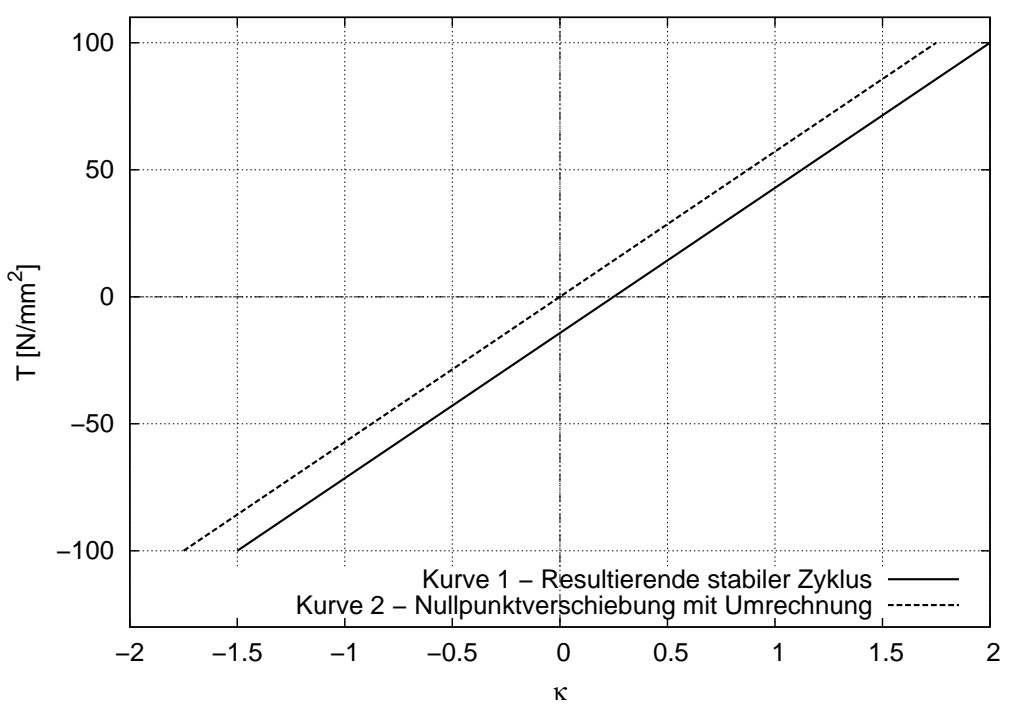

Bild 6.34: Nullpunktverschiebung der Messdaten bei Scherung.

Wie in Bild 6.34 verdeutlicht wird, ist demzufolge auch der Anstieg der beiden Kurven identisch. Dies stellt eine grundsätzliche Abgrenzung zur korrekten Umrechnung der Messdaten von Zugversuchen dar.

Im Hinblick auf die im Projekt verwendeten Standardversuche kann nunmehr die Auswirkung der Messdatenmanipulation auf die Identifikation für hyperelastische Stoffgesetze bewertet werden. Hierbei ist zu berücksichtigen, dass lediglich der Simple-Shear-Versuch einen Scherversuch darstellt, während die übrigen Belastungen den Zugversuchen zuzuordnen sind. Entsprechend des Umgangs mit den Messdaten sollen die Fälle 
- keine Nullpunktverschiebung,

- Nullpunktverschiebung ohne Umrechung, sowie

- Nullpunktverschiebung mit Umrechnung gemäß Gl. 6.20 und Gl. 6.22

miteinander verglichen werden.

1. Unabhängig von der Anpassungsgenauigkeit ist die Nullverschiebung von Messdaten ohne entsprechende Umrechnung physikalisch nicht korrekt und sollte deshalb grundsätzlich vermieden werden.

2. Bei Verwendung der Messkurven ohne Nullpunktverschiebung tritt gegenüber den anderen beiden Varianten im ersten Viertel der Kurvenverläufe zwangsläufig eine größere Abweichung zu den Messdaten auf. Dies ist jedoch unkritisch, da der ermittelte Parametersatz in diesem Bereich ohnehin nur eingeschränkte Gültigkeit besitzt (siehe Kap. 6.6.3).

3. Erfolgt die Anpassung anhand aller vier Standardversuche, führt die Anpassung an die Messdaten nach Nullpunktverschiebung mit Umrechnung zu einem vergleichsweise schlechten Identifikationsergebnis. Der Grund dafür liegt darin, dass die umgerechneten Messkurven der Zugversuche einen anderen Materialzustand repräsentieren als die umgerechneten Daten des Simple-Shear-Versuches. Beim Simple-Shear-Versuch findet ausschließlich eine Änderung der Eigenrichtungen statt. Zudem ist die Belastung im Gegensatz zu den Zugversuchen nicht einsinnig, weshalb sich eine andere Form der Anisotropie ausbildet. Um Vergleichbarkeit zwischen den umgerechneten Zugversuchen und dem Simple Shear-Versuch zu erreichen, müsste eine bereits durch den Zugversuch vorbelastete Probe geschert werden. Infolgedessen würde sich $\kappa$ ändern, da sich die Probendicke $d$ entsprechend Gl. 6.24 ändern würde. Da entsprechende Messungen aber nicht vorliegen, ist es günstiger, auf die Simple-Shear-Versuche zu verzichten.

4. Ein Verzicht auf die Simple-Shear-Versuche wiederum hätte einen merklichen Informationsverlust zur Folge, da eben dieser Versuch einen deutlichen Mehrwert an Information bezüglich des Materialverhaltens darstellt (vergl. Bild 6.23).

\subsubsection{Kriterien zum Vergleich der Lastfälle}

Aufgrund der begrenzten Gültigkeit identifizierter Parameter bezüglich der Beanspruchungsintensität eines Materialpunkts ist die Abstimmung der Belastungsamplituden der Standardversuche auf die im Bauteil typischen Belastungsintensitäten außerordentlich wichtig. In diesem Zusammenhang muss jedoch auch die Abstimmung der Belastungsamplituden der unterschiedlichen Experimente aufeinander betrachtet werden. Zu diesem Zweck sollen unterschiedliche Vergleichsmaße betrachtet und im Folgenden vorgestellt werden. 


\section{Vergleichsmaß Energiedichte}

Entsprechend des ODGEN-Ansatzes ergibt sich die Energiedichte $\hat{W}$ aus den Materialkennwerten und den Hauptstreckungen $\lambda_{i} \mathrm{zu}$ :

$$
\hat{W}=\sum_{n} \frac{\mu_{n}}{\alpha_{n}}\left(\lambda_{1}^{\alpha_{n}}+\lambda_{2}^{\alpha_{n}}+\lambda_{3}^{\alpha_{n}}-3\right) .
$$

Dabei stellen $\lambda_{1}, \lambda_{2}$ und $\lambda_{3}$ die Eigenwerte des rechten Strecktensors $\underline{\underline{U}}=\sqrt{\underline{\underline{C}}}$ dar. Die Zahlenwerte der folgenden Berechnungen basieren auf Materialdaten, welche durch die Firma Anvis bereitgestellt wurden. Um bei den Messungen die grobe Übereinstimmung zwischen der Belastungsintensität der verschiedenen Versuche zu gewährleisten, wird von einer jeweils gleichen Energiedichte ausgegangen. Zudem wird das Material als ideal inkompressibel betrachtet. Die Hauptstreckung $\lambda_{1}$ wird immer als Hauptzugrichtung angenommen. Auf deren Basis lässt sich die Energiedichte für die verschiedenen Belastungen ermitteln, welche im Folgenden angegeben sind.

\section{S2-Zugversuch}

Da es sich um einachsigen Zug handelt, ergeben sich

$$
\lambda_{2}=\lambda_{3}=\frac{1}{\sqrt{\lambda_{1}}} .
$$

Somit lässt sich die Energiedichte wie folgt zusammenfassen:

$$
\hat{W}=\sum_{n} \frac{\mu_{n}}{\alpha_{n}}\left(\lambda_{1}^{\alpha_{n}}+2\left(\frac{1}{\sqrt{\lambda_{1}}}\right)^{\alpha_{n}}-3\right) .
$$

\section{Biaxialer Zugversuch}

Beim biaxialen Zugversuch gilt aufgrund $\lambda_{2}=\lambda_{1}$ :

$$
\lambda_{3}=\frac{1}{\lambda_{1}^{2}} .
$$

Somit lässt sich die Energiedichte wie folgt zusammenfassen:

$$
\hat{W}=\sum_{n} \frac{\mu_{n}}{\alpha_{n}}\left(2 \lambda_{1}^{\alpha_{n}}+\left(\frac{1}{\lambda_{1}^{2}}\right)^{\alpha_{n}}-3\right) .
$$

\section{Plane-Strain-Zugversuch}

Der Plane-Strain-Zugversuch gleicht dem S2-Zugversuch, wobei $\lambda_{2}=1$ erzwungen wird. Demnach ergibt sich $\lambda_{3}$ zu:

$$
\lambda_{3}=\frac{1}{\lambda_{1}}
$$

Somit lässt sich die Energiedichte wie folgt zusammenfassen:

$$
\hat{W}=\sum_{n} \frac{\mu_{n}}{\alpha_{n}}\left(\lambda_{1}^{\alpha_{n}}+1+\left(\frac{1}{\lambda_{1}}\right)^{\alpha_{n}}-3\right) .
$$




\section{Simple-Shear-Versuch}

Beim Simple-Shear-Versuch muss auf das Schermaß $\kappa$ zurückgegriffen werden:

$$
\begin{aligned}
& \lambda_{1}=\sqrt{1+\frac{\kappa^{2}}{4}}+\frac{\kappa}{2}, \\
& \lambda_{2}=\sqrt{1+\frac{\kappa^{2}}{4}}-\frac{\kappa}{2}, \\
& \lambda_{3}=1 .
\end{aligned}
$$

Somit lässt sich die Energiedichte wie folgt zusammenfassen:

$$
\hat{W}=\sum_{n} \frac{\mu_{n}}{\alpha_{n}}\left(\left(\sqrt{1+\frac{\kappa^{2}}{4}}+\frac{\kappa}{2}\right)^{\alpha_{n}}+\left(\sqrt{1+\frac{\kappa^{2}}{4}}-\frac{\kappa}{2}\right)^{\alpha_{n}}+1-3\right) .
$$

Für die Ermittlung der Hauptstreckungen $\lambda_{1}$ wird eine gleichmäßige Aufteilung der zugehörigen logarithmischen Hauptdehnung $h$ gewählt, womit sich $\lambda_{1}$ wie folgt ergibt:

$$
\lambda_{1 i}=e^{h_{i}} \quad \text { mit } \quad i=1,2,3 .
$$

Ausgangsbasis ist der S2-Zugversuch, bei welchem für den mehrstufigen Versuch die Hauptdehnungen $h_{1}=0,3, h_{2}=0,6$ und $h_{3}=0,9$ angefahren werden.

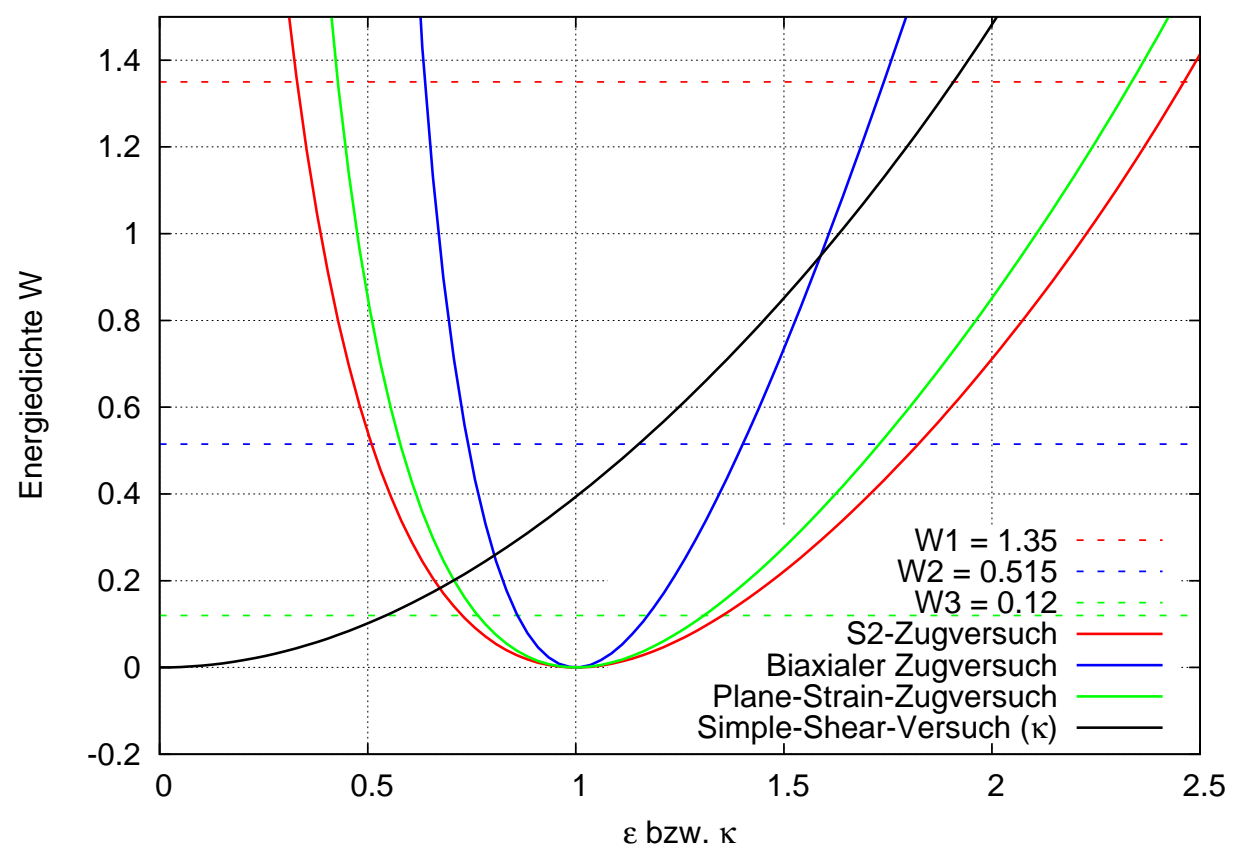

Bild 6.35: Streckungen für die unterschiedlichen Belastungen.

Die den angefahrenen Hauptdehnungen zugeordneten Streckungen für die unterschiedlichen Belastungen können Tabelle 6.2 entnommen werden. Zudem sind die entsprechenden Verläufe in Bild 6.35 grafisch veranschaulicht. 
Tabelle 6.2: Streckungen für die unterschiedlichen Belastungen.

\begin{tabular}{|c|c|c|c|c|c|}
\hline$h_{1}^{\text {Zug }}$ & $\hat{W}$ & $\lambda_{1}^{\text {Zug }}$ & $\lambda_{1}^{\text {Biax }}$ & $\lambda_{1}^{\text {Plane }}$ & $k^{\text {Simple }}$ \\
\hline 0,9 & 1,35 & 2,46 & 1,74 & 2,33 & 1,91 \\
0,6 & 0,52 & 1,82 & 1,40 & 1,73 & 1,15 \\
0,3 & 0,12 & 1,35 & 1,17 & 1,31 & 0,54 \\
\hline
\end{tabular}

\section{Geometrisches Vergleichsmaß}

Für den Fall, dass bezüglich der Materialparameter des zu identifizierenden Werkstoffes keinerlei Kenntnisse vorliegen, bietet sich die Verwendung eines geometrischen Vergleichsmaßes an. Im Folgenden wird ein Vergleichsmaß erläutert, welches auf dem Umformgrad $\bar{\lambda}$ basiert.

Die Zeitableitung des Umformgrades $\bar{\lambda}$ ergibt sich entsprechend Haupt (2002) nach:

$$
\begin{aligned}
\dot{\bar{\lambda}} & =\frac{1}{2} \sqrt{S p\left(\underline{\underline{C}} \cdot \underline{\underline{C}}^{-1}\right)^{2}} \\
& =\sqrt{\underline{\underline{D}} \cdot \underline{\underline{D}}}
\end{aligned}
$$

wobei $\underline{\underline{C}}$ wieder den Rechts-CAUCHY-GREEN-Tensor darstellt, welcher sich auf Basis des Deformationsgradienten $\underline{\underline{F}}$ entsprechend

$$
\underline{\underline{C}}=\underline{\underline{F}}^{T} \cdot \underline{\underline{F}} \quad \text { sowie } \quad \underline{\underline{D}}=\frac{1}{2}\left(\underline{\underline{L}}+\underline{\underline{L}}^{T}\right) \quad \text { mit } \quad \underline{\underline{L}}=\left(\underline{\underline{\dot{F}}} \cdot \underline{\underline{F}}^{-1}\right)
$$

ergibt. Auf Basis von Gl.6.39 lässt sich nach Haupt die akkumulierte Verzerrungsgeschwindigkeit $\dot{s}$ darstellen:

$$
\dot{s}=\sqrt{\frac{2}{3} \underline{\underline{D}} \cdot \cdot \underline{\underline{D}}}=\sqrt{\frac{2}{3}} \dot{\bar{\lambda}} \quad \text { bzw. } \quad s=\sqrt{\frac{2}{3}} \bar{\lambda} .
$$

Im Weiteren werden die vier verschiedenen Versuche in Bezug auf den Umformgrad $\bar{\lambda}$ betrachtet.

\section{S2-Zugversuch}

Aus

$$
\left[F_{a b}\right]=\left[\begin{array}{ccc}
\lambda_{1} & 0 & 0 \\
0 & \lambda_{1}^{-\frac{1}{2}} & 0 \\
0 & 0 & \lambda_{1}^{-\frac{1}{2}}
\end{array}\right]
$$

folgt mit

$$
\left[\dot{C}_{a b}\right]=\dot{\lambda}_{1}\left[\begin{array}{ccc}
2 \lambda_{1} & 0 & 0 \\
0 & -\lambda_{1}^{-2} & 0 \\
0 & 0 & -\lambda_{1}^{-2}
\end{array}\right] \quad \text { und } \quad\left[C_{a b}^{-1}\right]=\left[\begin{array}{ccc}
\lambda_{1}^{-2} & 0 & 0 \\
0 & \lambda_{1} & 0 \\
0 & 0 & \lambda_{1}
\end{array}\right]
$$


die Gleichung

$$
\dot{\bar{\lambda}}=\sqrt{\frac{3}{2}} \lambda_{1}^{-1} \dot{\lambda}_{1}
$$

Durch Integration erhält man somit

$$
\bar{\lambda}=\sqrt{\frac{3}{2}} \ln \lambda_{1} \quad \text { bzw. } \quad s=\ln \lambda_{1}
$$

\section{Biaxialer Zugversuch}

Aus

$$
\left[F_{a b}\right]=\left[\begin{array}{ccc}
\lambda_{1} & 0 & 0 \\
0 & \lambda_{1} & 0 \\
0 & 0 & \lambda_{1}^{-2}
\end{array}\right]
$$

folgt mit

$$
\left[\dot{C}_{a b}\right]=\dot{\lambda}_{1}\left[\begin{array}{ccc}
2 \lambda_{1} & 0 & 0 \\
0 & 2 \lambda_{1} & 0 \\
0 & 0 & -4 \lambda_{1}^{-5}
\end{array}\right] \quad \text { und } \quad\left[C_{a b}^{-1}\right]=\left[\begin{array}{ccc}
\lambda_{1}^{-2} & 0 & 0 \\
0 & \lambda_{1}^{-2} & 0 \\
0 & 0 & \lambda_{1}^{4}
\end{array}\right]
$$

die Gleichung

$$
\dot{\bar{\lambda}}=\sqrt{6} \lambda_{1}^{-1} \dot{\lambda}_{1}
$$

Durch Integration erhält man somit

$$
\bar{\lambda}=\sqrt{6} \ln \lambda_{1}
$$

\section{Plane-Strain-Zugversuch}

Aus

$$
\left[F_{a b}\right]=\left[\begin{array}{ccc}
\lambda_{1} & 0 & 0 \\
0 & 1 & 0 \\
0 & 0 & \lambda_{1}^{-1}
\end{array}\right]
$$

folgt mit

$$
\left[\dot{C}_{a b}\right]=\dot{\lambda}_{1}\left[\begin{array}{ccc}
2 \lambda_{1} & 0 & 0 \\
0 & 0 & 0 \\
0 & 0 & -2 \lambda_{1}^{-3}
\end{array}\right] \quad \text { und } \quad\left[C_{a b}^{-1}\right]=\left[\begin{array}{ccc}
\lambda_{1}^{-2} & 0 & 0 \\
0 & 1 & 0 \\
0 & 0 & \lambda_{1}^{2}
\end{array}\right]
$$

die Gleichung

$$
\dot{\bar{\lambda}}=\sqrt{2} \lambda_{1}^{-1} \dot{\lambda}_{1}
$$

Durch Integration erhält man somit

$$
\bar{\lambda}=\sqrt{2} \ln \lambda_{1}
$$




\section{Simple-Shear-Versuch}

Aus

$$
\left[F_{a b}\right]=\left[\begin{array}{ccc}
1 & \kappa & 0 \\
0 & 1 & 0 \\
0 & 0 & 1
\end{array}\right]
$$

folgt mit

$$
\left[\dot{C}_{a b}\right]=\dot{\kappa}\left[\begin{array}{ccc}
0 & 1 & 0 \\
1 & 2 \kappa & 0 \\
0 & 0 & 0
\end{array}\right] \quad \text { und } \quad\left[C_{a b}^{-1}\right]=\left[\begin{array}{ccc}
1+\kappa^{2} & -\kappa & 0 \\
-\kappa & 1 & 0 \\
0 & 0 & 1
\end{array}\right]
$$

die Gleichung

$$
\dot{\bar{\lambda}}=\frac{1}{\sqrt{2}} \dot{\kappa}
$$

Durch Integration erhält man somit

$$
\bar{\lambda}=\frac{1}{\sqrt{2}} \kappa=\frac{1}{\sqrt{2}} \frac{K}{h} .
$$

\section{Vergleich der Versuche mittels des Umformgrades}

Durch die Verwendung des Umformgrades lassen sich die Hauptstreckungen für die Biaxial-, Plane-Strain- und Simple-Shear-Versuche auf Basis des Zugversuches sehr leicht ermitteln, wenn für alle Versuche ein identischer Umformgrad gefordert wird:

$$
\begin{array}{ll}
\bar{\lambda} & =\sqrt{\frac{3}{2}} \ln \lambda_{1}^{\text {Zug }}, \\
\lambda_{1}^{\text {Biax }} & =\sqrt{\lambda_{1}^{Z u g}}, \\
\lambda_{1}^{\text {Plane }} & =\left(\lambda_{1}^{\text {Zug }}\right)^{\frac{\sqrt{3}}{2}}, \\
\kappa^{\text {Simple }} & =\sqrt{3} \ln \lambda_{1}^{\text {Zug }} .
\end{array}
$$

Für die in den Versuchen genutzten Zugdehnungen ergeben sich die in Tabelle 6.3 aufgeführten Werte.

Tabelle 6.3: Streckungen für die unterschiedlichen Belastungen.

\begin{tabular}{|c|c|c|c|c|c|c|c|c|}
\hline$h_{1}^{\text {Zug }}$ & $\bar{\lambda}$ & $\lambda_{1}^{\text {Zug }}$ & $\lambda_{2}^{\text {Zug }}$ & $\lambda_{1}^{\text {Biax }}$ & $\lambda_{3}^{\text {Biax }}$ & $\lambda_{1}^{\text {Plane }}$ & $\lambda_{3}^{\text {Plane }}$ & $\kappa^{\text {Simple }}$ \\
\hline 0,9 & 1,1023 & 2,4596 & 0,6376 & 1,5683 & 0,4066 & 2,1802 & 0,4587 & 1,5588 \\
0,6 & 0,7348 & 1,8221 & 0,7408 & 1,3499 & 0,5488 & 1,6814 & 0,5947 & 1,0392 \\
0,3 & 0,3674 & 1,3499 & 0,8607 & 1,1618 & 0,7408 & 1,2967 & 0,7712 & 0,5196 \\
\hline
\end{tabular}




\section{Vorteile eines Beanspruchungsmaßes}

Falls für den zu prüfenden Gummiwerkstoff keinerlei Abschätzungen der Materialparameter auf Basis ähnlicher Werkstoffe mit bereits bekannten Materialparametern zur Verfügung stehen, sollte ein Verfahren mit einem ausschließlich auf Verformung basierenden Vergleichsmaß genutzt werden. Ist jedoch eine grobe Abschätzung der Parameter möglich, bietet ein Vergleichsmaß wie die Energiedichte durchaus Vorteile.

Der Spannungsverlauf in Bild 6.36 zeigt deutlich, dass die identifizierten Materialparameter stark von der gewählten Streckung abhängen. Gleiches gilt für die verbleibende Restdehnung, welche mit steigender Streckungsamplitude ebenfalls zunimmt. Bei der Nutzung unterschiedlicher Experimente muss deshalb darauf geachtet werden, dass diese miteinander vergleichbar sind. Eine Möglichkeit dafür stellt, wie vorab beschrieben, die Energiedichte $\hat{W}$ dar. Es liegt deshalb nahe, die Intensität der Werkstoffbelastung in Form der Energiedichte als vergleichendes Maß zu nutzen. Denn nicht die Streckung an sich, sondern die auf Basis der Materialparameter daraus resultierende Beanspruchung des Werkstoffes lässt diesen versagen.

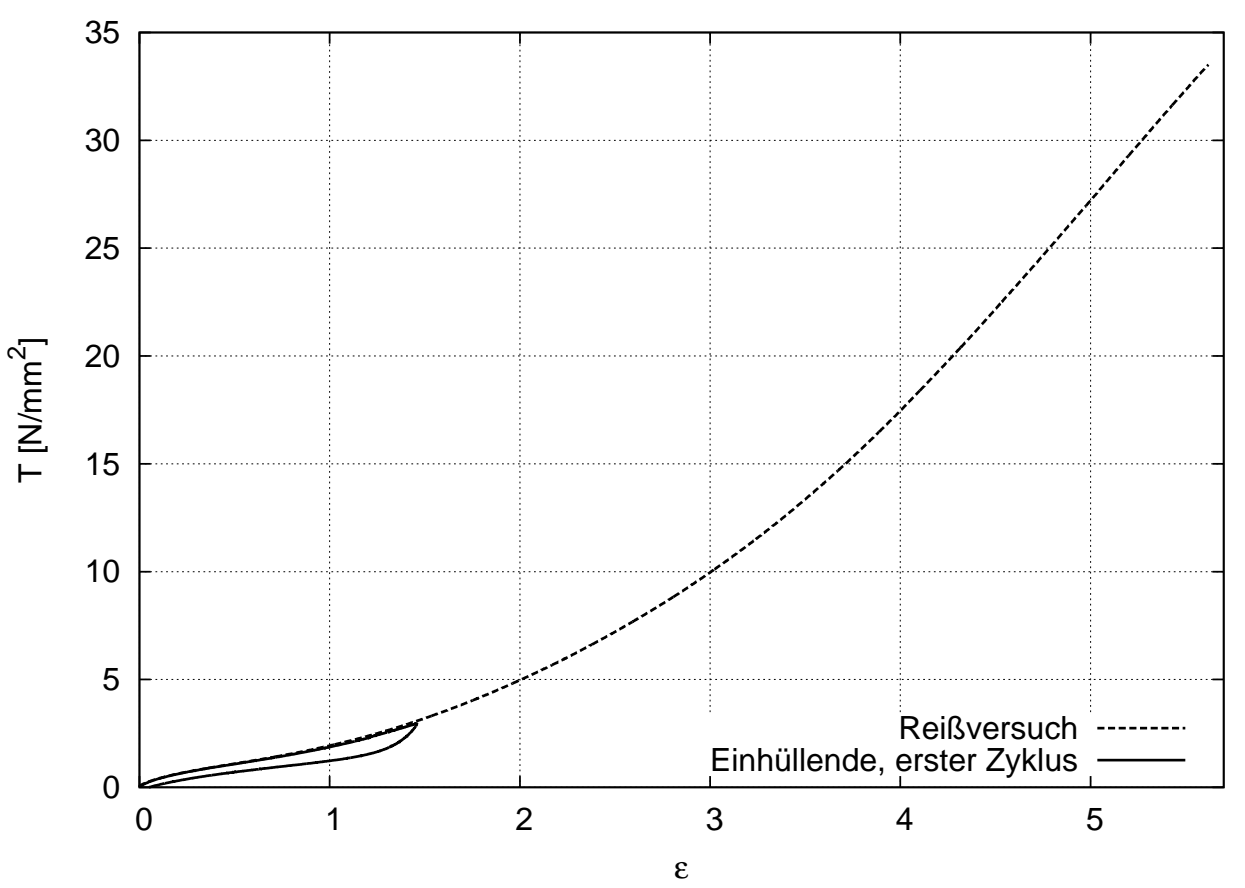

Bild 6.36: Vergleich des Reißversuches und des ersten Zyklus der Einhüllenden.

Aufgrund des nichtlinearen Materialverhaltens führt die Verwendung der Energiedichte als vergleichende Größe bezüglich der Versuche zu unterschiedlichen Verhältnissen zwischen den zugeordneten Hauptstreckungen verschiedener Belastungsstufen. Dies ist jedoch nicht zwangsläufig ein Nachteil, da wie oben beschrieben nicht die Streckungen allein entscheidend sind. 


\subsubsection{Durchführung der Identifikation}

Basierend auf den Vorbetrachtungen lassen sich die Eckpunkte für die Identifikation mit Standardprobekörpern wie folgt zusammenfassen:

- Die Identifikation erfolgt für das YEOH-Materialgesetz (Yeoh, 1993), das OGDENMaterialgesetz (Ogden, 1972) und MORPH in RepRicht (Freund und Ihlemann, 2010).

- Für die Identifikation der YeOH- und OGDEn-Parameter werden die einstufigen Versuche der vier Belastungsarten verwendet, wobei jedes Experiment drei Mal wiederholt und dann gemittelt wird. Abweichend davon werden bei der Anpassung von MORPH in RepRicht die dreistufigen Versuche benötigt. In allen Fällen finden dabei jeweils ausschließlich die stationären Zyklen Berücksichtigung. Die Belastung erfolgt weggesteuert.

- Eine Nullpunktverschiebung oder andere Modifikationen der Messdaten finden nicht statt.

- Als Zielfunktionswert wird $\overline{\text { GFK }}$ (vergl. Kap. 3.2) genutzt.

\section{Identifikation für das YEOH-Stoffgesetz}

Unter Berücksichtigung der vorgenannten Rahmenbedingungen werden die Materialparameter des YEOH-Stoffgesetzes mittels der Standardprobekörper identifiziert. Ausgehend von einem willkürlich gewählten Startparametersatz führt die Minimierung der kombinierten gewichteten Fehlerquadrat-Kennzahl GFK auf die in Tabelle 6.4 dargestellten Parameter.

Tabelle 6.4: Ergebnis der YEOH-Parameter-Identifikation nach 4 Schritten.

\begin{tabular}{|l|l|l|c|c|}
\hline & $\mathrm{C}_{10}[\mathrm{MPa}]$ & $\mathrm{C}_{20}[\mathrm{MPa}]$ & $\mathrm{C}_{30}[\mathrm{MPa}]$ & $\overline{\mathrm{GFK}}$ \\
\hline Startparameter & 1,0 & 1,0 & 1,0 & 27,328 \\
Identifizierte Parameter & 0,352 & $-0,0071$ & 0,00386 & $2,240 \cdot 10^{-3}$ \\
\hline
\end{tabular}

In diesem Kontext soll das Kriterium der gewichteten Fehlerquadrat-Kennzahl bezüglich der Anpassung der Simulationsdaten an die Messdaten für die einzelnen Experimente der Homogen-Identifikation bewertet werden. Die dafür notwendigen Daten werden den LogDateien entnommen, welche im Rahmen der Identifikation durch SPC-Opt angelegt werden.

Tabelle 6.5: GFK-Ermittlung für identifizierte YEOH-Parameter.

\begin{tabular}{|l|c|c|c|c|}
\hline & $\sum_{i=1}^{n_{L}}\left(\bar{y}_{i}-M_{i}\right)^{2}$ & $n_{L}$ & $\max (\bar{y})-\min (\bar{y})$ & $\mathrm{GFK}$ \\
\hline S2-Zugversuch & 1,025 & 95 & 2,34 & $1,971 \cdot 10^{-3}$ \\
Biaxialer Zugversuch & 1,108 & 95 & 1,60 & $4,554 \cdot 10^{-3}$ \\
Plane-Strain-Zugversuch & 0,909 & 95 & 2,03 & $2,323 \cdot 10^{-3}$ \\
Simple-Shear-Versuch & 0,765 & 98 & 3,02 & $0,856 \cdot 10^{-3}$ \\
\hline
\end{tabular}



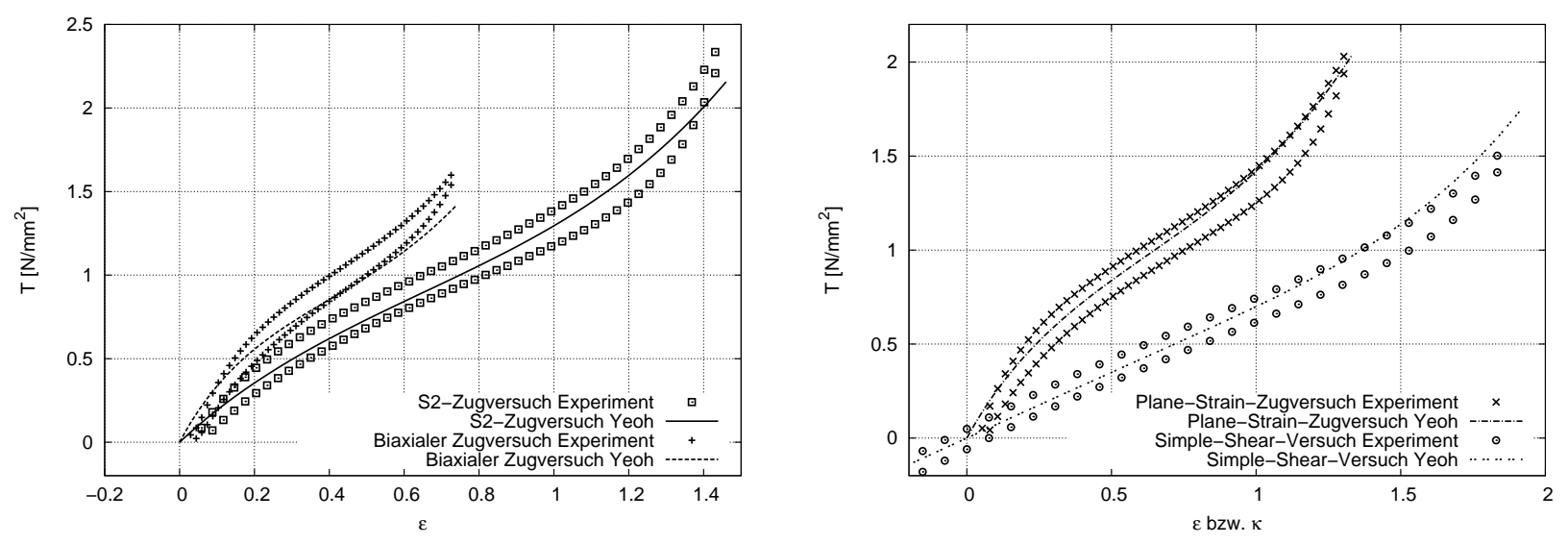

Bild 6.37: Vergleich der Experimente mit den zugehörigen Simulationen bei Verwendung der anhand homogener Standardversuche identifizierten YEOH-Parameter.

Die Berechnung der GFK der verschiedenen Versuche, mit denen nach Gl. 3.18 der Zielfunktionswert in Form von GFK gebildet wird, geht aus Tabelle 6.5 hervor. Beim Vergleich der einzelnen GFK für die verschiedenen Experimente mit der visuellen Einordnung der Anpassungsqualität entsprechend Bild 6.37 wird eine gute Übereinstimmung ersichtlich. Während die Simple-Shear-Versuche besonders gut angepasst werden, ist die Diskrepanz zwischen den Messdaten und der Simulation für den biaxialen Zugversuch deutlich höher.

In SPC-Opt besteht die Möglichkeit zur Ausgabe der Korrelationsmatrix. Sie basiert für Probleme der kleinsten Quadrate auf der approximierten Hessematrix und kann bei der Detektion voneinander abhängiger Parameter hilfreich sein. Ein Hinweis auf starke Korrelation liegt dann vor, wenn der Betrag eines Elementes, welches nicht auf der Hauptdiagonalen liegt, nahe eins ist. Da die in Tabelle 6.6 dargestellte Korrelationsmatrix keine kritischen Werte beinhaltet, kann die Korrelationen einzelner Parameter ausgeschlossen werden.

Tabelle 6.6: Korrelationsmatrix für identifizierte YEOH-Parameter.

\begin{tabular}{|l|l|c|c|}
\hline & $\mathrm{C}_{10}$ & $\mathrm{C}_{20}$ & $\mathrm{C}_{30}$ \\
\hline $\mathrm{C}_{10}$ & 1 & $-0,757$ & 0,489 \\
$\mathrm{C}_{20}$ & & 1 & $-0,919$ \\
$\mathrm{C}_{30}$ & & & 1 \\
\hline
\end{tabular}

Aufgrund der hohen Stabilität der FEM-Simulationen, auch bei ungünstigen Startparametervariationen, sowie der schnellen Konvergenz der Identifikation, eignet sich das YEOHStoffgesetz für die Identifikation anhand bauteilnaher Probekörper.

\section{Identifikation für das OGDEN-Stoffgesetz}

Die Anpassung des OGDEN-Stoffgesetzes erfolgt analog zur Vorgehensweise bei der Identifikation der YEOH-Parameter. Entsprechend der grundsätzlichen Betrachtungen wird die Identifikation für zwei OGDEN-Terme durchgeführt. Als Startwerte stehen hier die OGDENParameter eines bereits bekannten Materials mit vergleichbaren Eigenschaften zur Verfügung. In Tabelle 6.7 sind die identifizierten Parameter dargestellt. Diese unterscheiden sich 
offenbar nur geringfügig von den Startparametern, weshalb auch die Verringerung des Zielfunktionswertes recht klein ist. Der Zielfunktionswert am Ende der Identifikation entspricht etwa dem der YEOH-Identifikation (Tabelle 6.4).

Tabelle 6.7: Ergebnis der OGDEN-Parameter-Identifikation nach 7 Schritten.

\begin{tabular}{|l|c|c|c|c|c|}
\hline & $\mu_{1}[\mathrm{MPa}]$ & \multicolumn{1}{|c|}{$\alpha_{1}$} & $\mu_{2}[\mathrm{MPa}]$ & $\alpha_{2}$ & $\overline{\mathrm{GFK}}$ \\
\hline Startparameter & 0,03 & 5,0 & 12,0 & 0,1 & $3,298 \cdot 10^{-3}$ \\
Identifizierte Parameter & 0,0586 & 4,59 & 11,74 & 0,0894 & $2,185 \cdot 10^{-3}$ \\
\hline
\end{tabular}

Bild 6.38 zeigt den Vergleich der Experimente mit den Verläufen der zugehörigen Simulationen. Bereits während der Vorbetrachtungen zeigte sich, dass die Verwendung von mehr als zwei OGDEn-Termen keine deutliche Verbesserung der Anpassung liefert. Eine entsprechende Parameteridentifikation ist deshalb an dieser Stelle nicht zielführend.
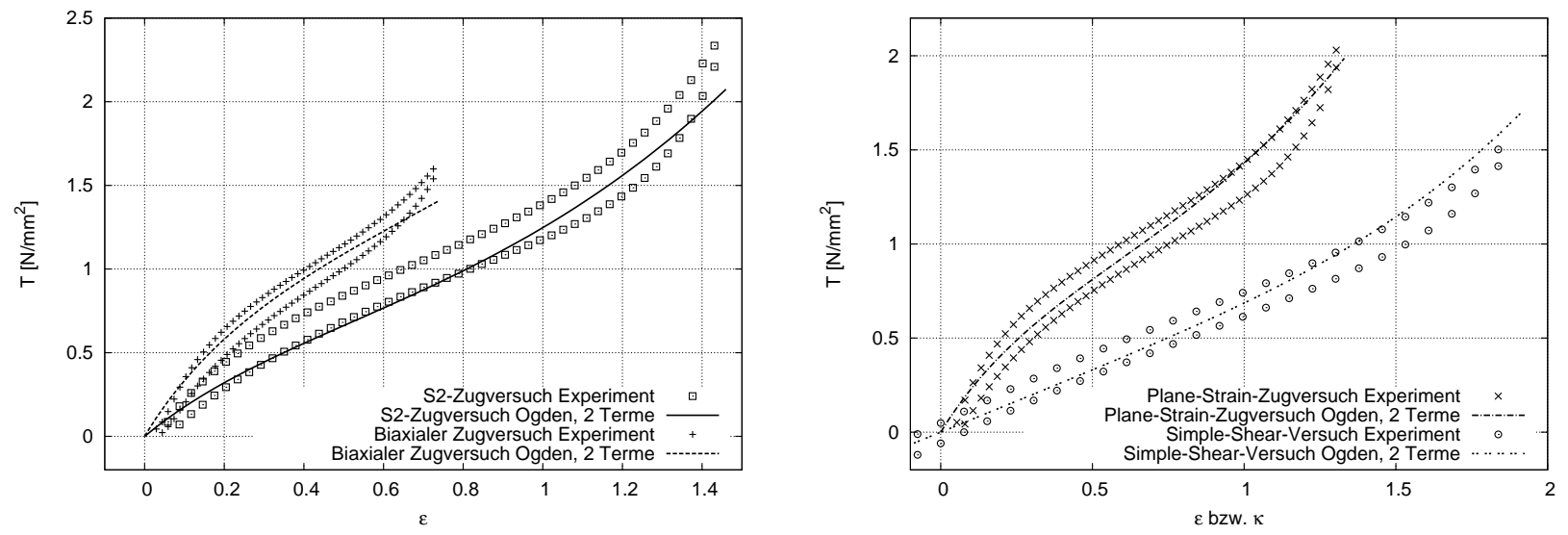

Bild 6.38: Vergleich der Experimente mit den zugehörigen Simulationen bei Verwendung der anhand homogener Standardversuche identifizierten OGDEN-Parameter.

Im Gegensatz zur YEOH-Identifikation beinhaltet die Korrelationsmatrix in Tabelle 6.8 durchaus kritische Werte nahe eins. Das bedeutet, dass für die identifizierten Parameter offenbar zwischen den Basen der OGDEN-Terme und ihren zugehörigen Exponenten eine starke Korrelation auftritt. Um diese Annahme validieren zu können, soll im Weiteren eine Startparametervariation durchgeführt werden, um die identifizierten Parametervektoren bei unterschiedlichen Startparametervektoren miteinander vergleichen zu können.

Tabelle 6.8: Korrelationsmatrix für identifizierte OGDEN-Parameter.

\begin{tabular}{|c|c|c|c|c|}
\hline & $\mu_{1}$ & $\alpha_{1}$ & $\mu_{2}$ & $\alpha_{2}$ \\
\hline$\mu_{1}$ & 1 & $\mathbf{- 0 , 9 8 8}$ & 0,317 & 0,387 \\
$\alpha_{1}$ & & 1 & $-0,265$ & 0,330 \\
$\mu_{2}$ & & & 1 & $-\mathbf{0 , 9 9 7}$ \\
$\alpha_{2}$ & & & & 1 \\
\hline
\end{tabular}


Um die Startparametervariation durchführen zu können, müssen zunächst Bereichsgrenzen der OGDEN-Parameter definiert werden, innerhalb derer die Parameter variiert werden (Tabelle 6.9).

Tabelle 6.9: Gewählter Bereich für die Startparametervariation der OGDEN-Parameter.

\begin{tabular}{|c|c|c|}
\hline & Untergrenze & Obergrenze \\
\hline$\mu_{1}[\mathrm{MPa}]$ & $-1,0$ & 2,0 \\
$\alpha_{1}$ & $-8,0$ & 8,0 \\
$\mu_{2}[\mathrm{MPa}]$ & $-5,0$ & 20,0 \\
$\alpha_{2}$ & $-1,0$ & 2,0 \\
\hline
\end{tabular}

Da für jede Parametervariation eine vollständige Identifikation durchgeführt werden muss, wird die Anzahl der Variationen auf 10 beschränkt. Entsprechend Kap. 5 bietet sich die Nutzung des Latin-Hypercube-Verfahrens in Kombination mit dem Simulated-AnnealingVerfahren als Sampling-Methode an. Als zugehöriges Design-Kriterium wird das auf Abstandsmessungen basierende Kriterium nach AudzE-EGLAIS gewählt. Die auf dieser Basis erzeugten Startparameter sind in Tabelle 6.10 dargestellt.

Tabelle 6.10: Startwerte der OGDEN-Parameter bei Startparametervariation.

\begin{tabular}{|l|r|r|r|r|c|}
\hline & \multicolumn{1}{|c|}{$\mu_{1}[\mathrm{MPa}]$} & \multicolumn{1}{c|}{$\alpha_{1}$} & $\mu_{2}[\mathrm{MPa}]$ & \multicolumn{1}{c|}{$\alpha_{2}$} & $\overline{\mathrm{GFK}}$ \\
\hline Parametersatz 1 & 1,25 & $-4,0$ & $-3,75$ & 0,65 & 41,20 \\
Parametersatz 2 & 1,85 & 0,8 & 8,75 & 1,55 & 13,92 \\
Parametersatz 3 & $-0,25$ & 5,6 & 1,25 & $-0,25$ & 5,622 \\
Parametersatz 4 & $-0,85$ & 2,4 & 11,25 & 1,25 & 7,007 \\
Parametersatz 5 & 0,95 & 7,2 & 16,25 & 0,35 & 526,6 \\
Parametersatz 6 & 0,35 & 4,0 & $-1,25$ & 1,85 & 0,161 \\
Parametersatz 7 & $-0,55$ & $-5,6$ & 3,75 & 0,05 & 80,11 \\
Parametersatz 8 & 1,55 & $-0,8$ & 6,25 & $-0,85$ & 5,434 \\
Parametersatz 9 & 0,05 & $-2,4$ & 18,75 & $-0,55$ & 11,06 \\
Parametersatz 10 & 0,65 & $-7,2$ & 13,75 & 0,95 & NN \\
\hline
\end{tabular}

Die Auswertung der identifizieren Parameter entsprechend Tabelle 6.11 zeigt, dass mehrere Startparameterkombinationen nicht in ein lokales Minimum innerhalb des zulässigen Bereiches führen, sondern an den Grenzen (GW) hängen bleiben. Des Weiteren gibt es Parameterkombinationen, bei denen selbst die Simulation der homogenen Belastung an nur einem Element nicht konvergiert (Parametersatz 10). In den Fällen, bei denen ein lokales Minimum erreicht wird, liegen die entsprechenden Zielfunktionswerte zwar nah beieinander, die zugehörigen identifizierten Parametervektoren unterscheiden sich jedoch deutlich. Aufgrund der Korrelation zwischen den Basen und Exponenten der OGDEN-Terme und auftretenden Konvergenzproblemen bei der Simulation der radialen und kardanischen Belastung am Bauteil wird bei der Identifikation anhand bauteilnaher Probekörper das OGDEN-Materialgesetz nicht betrachtet. 
Tabelle 6.11: Identifizierte OGDEN-Parameter bei Startparametervariation.

\begin{tabular}{|l|c|c|c|c|c|}
\hline & $\mu_{1}[\mathrm{MPa}]$ & $\alpha_{1}$ & $\mu_{2}[\mathrm{MPa}]$ & $\alpha_{2}$ & $\overline{\mathrm{GFK}}$ \\
\hline Parametersatz 1 & 0,3648 & $-2,0230$ & $-5,0(\mathrm{GW})$ & $-0,5437$ & $19,42 \cdot 10^{-3}$ \\
Parametersatz 2 & 0,08335 & 4,1958 & 7,5551 & 0,1290 & $2,221 \cdot 10^{-3}$ \\
Parametersatz 3 & 0,08552 & 4,1761 & 10,029 & 0,0961 & $2,221 \cdot 10^{-3}$ \\
Parametersatz 4 & $-1,0(\mathrm{GW})$ & 0,99467 & 1,9511 & 1,3939 & $5,778 \cdot 10^{-3}$ \\
Parametersatz 5 & 0,09501 & 4,0694 & 16,959 & 0,05489 & $2,232 \cdot 10^{-3}$ \\
Parametersatz 6 & 0,06143 & 4,4781 & 3,2430 & 0,3312 & $2,219 \cdot 10^{-3}$ \\
Parametersatz 7 & 0,45201 & $-0,2395$ & 0,8054 & $2,0(\mathrm{GW})$ & $3,726 \cdot 10^{-3}$ \\
Parametersatz 8 & 1,9993 & $-0,0462$ & 0,7994 & $2,0(\mathrm{GW})$ & $3,674 \cdot 10^{-3}$ \\
Parametersatz 9 & $2,0(\mathrm{GW})$ & $-0,8303$ & 18,091 & 0,2015 & $11,91 \cdot 10^{-3}$ \\
Parametersatz 10 & $\mathrm{NN}$ & $\mathrm{NN}$ & $\mathrm{NN}$ & $\mathrm{NN}$ & $\mathrm{NN}$ \\
\hline
\end{tabular}

\section{Identifikation für MORPH in RepRicht}

Im Unterschied zu den Ansätzen nach YEOH und OGDEN können durch MORPH in RepRicht auch inelastische Effekte abgebildet werden. Um die entsprechenden acht Parameter erfolgreich zu identifizieren, genügen die Informationen der einstufigen Versuche nicht mehr. Stattdessen werden die quasistationären Zyklen der dreistufigen Versuche herangezogen (vergl. Bild 6.29). Bezüglich der Festsetzung der Startparameter wurde auf die Arbeit von Ihlemann (2003) zurückgegriffen. In dieser wurde für ein gefülltes NR-basiertes Material ein entsprechender Parametersatz identifiziert, wenn auch für die tensorielle Variante von Morph. Da die Materialantworten von MORPH in RepRicht und dem tensoriellen MoRPH bei Verwendung des gleichen Parametersatzes voneinander abweichen, wurden diese in (Freund, 2013) entsprechend umgerechnet. Die Parameter werden auch im Weiteren den Ausgangspunkt der Stoffgesetzanpassungen für MORPH in RepRicht darstellen.

Das Ergebnis der Anpassung sowie die entsprechenden GFK sind in Tabelle 6.12 und Tabelle 6.13 angegeben. Die in den Experimenten gemessenen Spannungen der für die Identifikation verwendeten quasistationären Zyklen sind in Bild 6.39 grafisch dargestellt. Darüber hinaus sind auch die Verläufe der entsprechenden FEM-Simulationen bei Verwendung der identifizierten Parameter $\underline{p}_{\text {Homogen }}$ enthalten. Auch hier zeigt sich beim Vergleich der Zahlenwerte der GFK mit den zugehörigen visuellen Abbildungen, dass das Kriterium zur Bewertung der Anpassungsgenauigkeit gut geeignet ist.

Tabelle 6.12: Startparameter und anhand von Standardprobekörpern bei Minimierung von $\overline{\text { GFK }}$ für MORPH in RepRicht identifizierte Parameter.

\begin{tabular}{|l|c|c|c|c|c|c|c|c|}
\hline & $p_{1}[\mathrm{MPa}]$ & $p_{2}[\mathrm{MPa}]$ & \multicolumn{1}{|c|}{$p_{3}$} & $p_{4}$ & $p_{5}[\mathrm{MPa}]$ & $p_{6}$ & $p_{7}$ & $p_{8}[\mathrm{MPa}]$ \\
\hline$\underline{p}_{\text {Start }}$ & 0,0109 & 0,408 & 0,421 & 6,850 & 0,00560 & 5,54 & 5,84 & 0,1170 \\
$\underline{p}_{\text {Homogen }}$ & 0,1366 & 0,3271 & 0,6341 & 11,208 & 0,000979 & 9,052 & 8,088 & 0,04861 \\
\hline
\end{tabular}


Tabelle 6.13: GFK für die Identifikation anhand von Standardprobekörpern bei Minimierung von $\overline{\text { GFK }}$ für MoRPH in RepRicht nach 26 Schritten.

\begin{tabular}{|l|c|c|}
\hline & $\operatorname{GFK}\left(\underline{p}_{\text {Start }}\right)$ & $\mathrm{GFK}\left(\underline{p}_{\text {Homogen }}\right)$ \\
\hline S2-Zugversuch & $2,455 \cdot 10^{-3}$ & $0,306 \cdot 10^{-3}$ \\
Biaxialer Zugversuch & $2,689 \cdot 10^{-3}$ & $1,264 \cdot 10^{-3}$ \\
Plane-Strain-Zugversuch & $2,452 \cdot 10^{-3}$ & $0,374 \cdot 10^{-3}$ \\
Simple-Shear-Versuch & $2,687 \cdot 10^{-3}$ & $0,600 \cdot 10^{-3}$ \\
Kombiniert & $2,570 \cdot 10^{-3}$ & $0,587 \cdot 10^{-3}$ \\
\hline
\end{tabular}

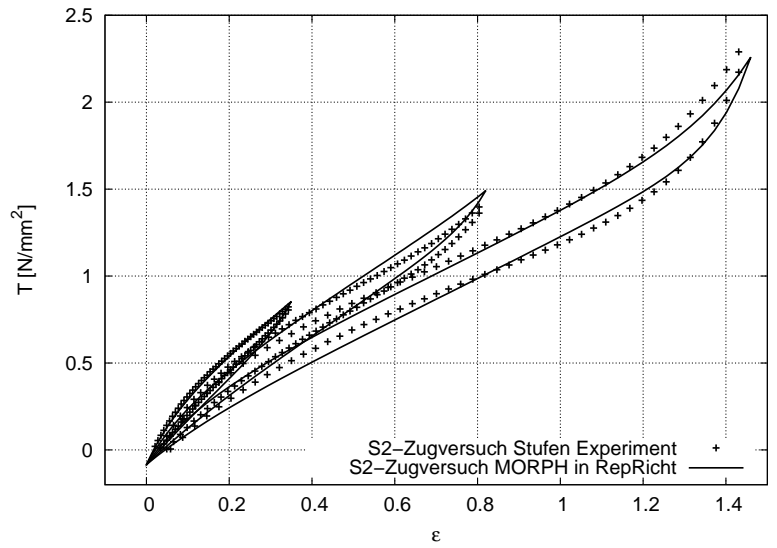

(a) S2-Zugversuch

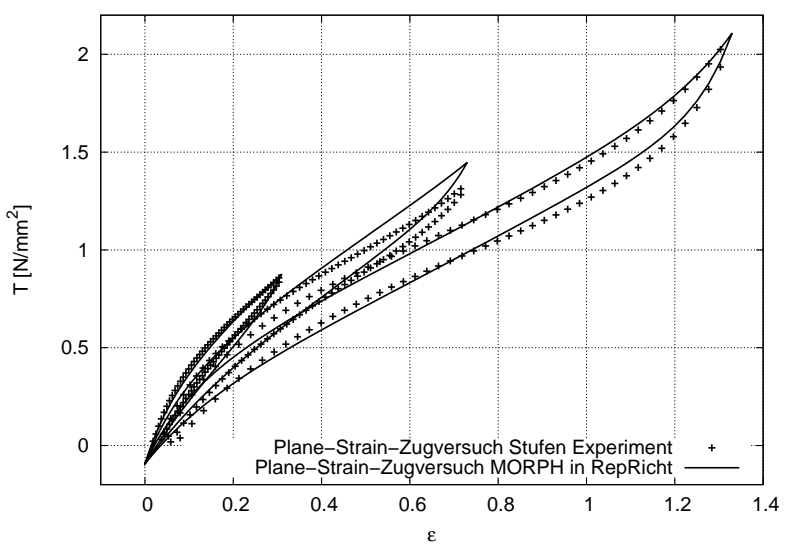

(c) Plane-Strain-Zugversuch

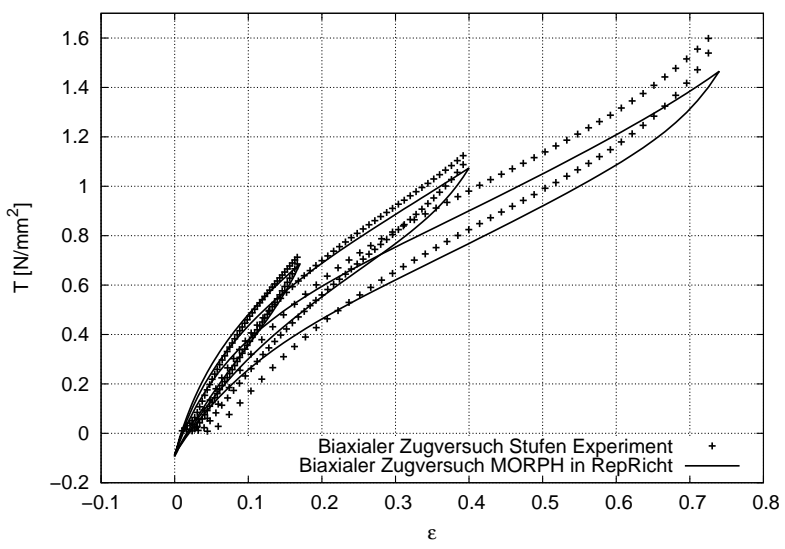

(b) Biaxialer Zugversuch

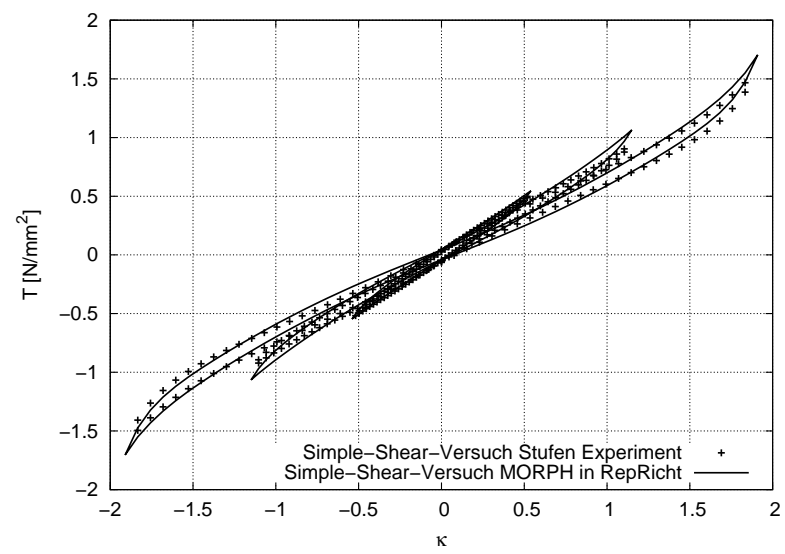

(d) Simple-Shear-Versuch

Bild 6.39: Vergleich der Experimente mit den zugehörigen Simulationen bei Verwendung der anhand homogener Standardversuche identifizierten Parameter für MORPH in RepRicht.

Bei Betrachtung aller vier Versuche wird sichtbar, dass das Materialverhalten aus dem biaxialen Zugversuch deutlich von dem der anderen Versuche abweicht. Dies erklärt sich höchstwahrscheinlich durch Messungenauigkeiten, welche infolge der Art des Versuchsaufbaus entstehen. Aufgrund dessen ist zu überlegen, ob lediglich die übrigen drei Belastungsarten bei der Identifikation verwendet werden sollten. Eine solche Vorgehensweise würde zu den in Tabelle 6.14 abgebildeten GFK der drei für die Anpassung verwendeten Versuche sowie der entsprechenden kombinierten GFK führen. Die Anpassungsgenauigkeit bei Auslassen des biaxialen Zugversuches verbessert sich demnach deutlich. Es ist jedoch zu beachten, dass 
der Verzicht auf diese Informationen die Wahrscheinlichkeit für eine Korrelation zwischen zwei oder mehr Parametern bzw. die Anzahl lokaler Minima der Zielfunktion erhöht. Eine stichprobenartige Kontrolle ergab, dass insbesondere bei der Wahl schlechter Startparameter das Minimum ohne Berücksichtigung der biaxialen Belastung nicht immer gefunden wird, während demgegenüber die Identifikation anhand aller vier Versuche für die geprüften Parametervariationen in jedem Fall das Minimum trifft.

Tabelle 6.14: GFK für die Identifikation anhand von Standardprobekörpern bei Minimierung von $\overline{\text { GFK }}$ für MORPH in RepRicht.

\begin{tabular}{|l|c|c|}
\hline & $\mathrm{GFK}\left(\underline{p}_{\text {Start }}\right)$ & $\mathrm{GFK}\left(\underline{p}_{\text {Homogen }}\right)$ \\
\hline S2-Zugversuch & $2,452 \cdot 10^{-3}$ & $0,199 \cdot 10^{-3}$ \\
Plain-Strain-Zugversuch & $2,464 \cdot 10^{-3}$ & $0,198 \cdot 10^{-3}$ \\
Simple-Shear-Versuch & $2,682 \cdot 10^{-3}$ & $0,517 \cdot 10^{-3}$ \\
Kombiniert & $2,532 \cdot 10^{-3}$ & $0,288 \cdot 10^{-3}$ \\
\hline
\end{tabular}

Die Kontrolle der Korrelationsmatrix in Tabelle 6.15zeigt keine Auffälligkeiten. Es ist jedoch nicht auszuschließen, dass es bei der doch erheblichen Anzahl von acht Parametern zwar keine direkte Korrelation zwischen zwei Parametern, sehr wohl aber eine komplexere Abhängigkeit zwischen mehreren Parametern gibt.

Tabelle 6.15: Korrelationsmatrix der identifizierten Parameter für MORPH in RepRicht.

\begin{tabular}{|c|c|c|c|c|c|c|c|c|}
\hline & $p_{1}$ & $p_{2}$ & $p_{3}$ & $p_{4}$ & $p_{5}$ & $p_{6}$ & $p_{7}$ & $p_{8}$ \\
\hline$p_{1}$ & 1 & $-0,829$ & 0,919 & 0,337 & $-0,403$ & $-0,402$ & 0,038 & $-0,105$ \\
\hline$p_{2}$ & & 1 & $-0,563$ & $-0,155$ & 0,388 & 0,356 & $-0,136$ & $-0,061$ \\
\hline$p_{3}$ & & & 1 & 0,350 & $-0,362$ & $-0,400$ & $-0,095$ & $-0,182$ \\
\hline$p_{4}$ & & & & 1 & $-0,265$ & $-0,225$ & 0,174 & $-0,586$ \\
\hline$p_{5}$ & & & & & 1 & 0,962 & $-0,262$ & 0,138 \\
\hline$p_{6}$ & & & & & & 1 & 0,011 & 0,191 \\
\hline$p_{7}$ & & & & & & & 1 & 0,163 \\
\hline$p_{8}$ & & & & & & & & 1 \\
\hline
\end{tabular}

Im Gegensatz zu den elastischen Stoffgesetzen kann das Materialverhalten mit MORPH in RepRicht deutlich besser abgebildet werden. Dies wird beim Vergleich der kombinierten GFK in Tabelle 6.13 mit Tabelle 6.4 bzw. Tabelle 6.7 deutlich.

\subsection{Identifikation anhand bauteilnaher Probekörper}

Während der frühen Phase eines Entwicklungsprozesses steht die exakte Abbildung des Materialverhaltens häufig nicht an vorderster Stelle. Sobald jedoch die möglichst genauen Wiedergabe der Realität durch FEM-Simulationen im Mittelpunkt steht, können die Nachteile der Standardprobekörper nicht mehr vernachlässigt werden. Wie in Kap. 6.3 beschrieben, 
wirkt sich neben dem Herstellungsprozess auch die geometrische Form der Bauteile auf deren Materialeigenschaften aus. Im Hinblick auf die Fahrwerksbuchse muss berücksichtigt werden, dass infolge der unterschiedlichen Abkühlungszeiten der Materialpunkte der eingespritzten Gummimischung während des Fertigungsprozesses bereits nach dem Abkühlen keine homogenen Materialeigenschaften vorliegen. Dementsprechend wäre für jeden Materialpunkt ein gesonderter Materialparametersatz zu ermitteln, um auf Basis des gewählten Stoffgesetzes das Materialverhalten möglichst gut nachzubilden. Da dies in der Praxis jedoch nicht möglich ist, werden diejenigen Materialparameter gesucht, welche einen guten Kompromiss für das Verhalten aller Materialbereiche darstellen. Die Anpassung an Standardprobekörper zur Identifikation dieses "gemittelten" Parametersatzes ist vor diesem Hintergrund nur eingeschränkt nutzbar, da sich der Vulkanisationsprozess bezüglich Druck und Temperaturverlauf grundlegend vom Bauteil unterscheidet. Vielmehr erscheint es sinnvoll, die Identifikation für die jeweilige Problemstellung separat zu betrachten und sowohl hinsichtlich des Abkühlprozesses als auch der Belastungen am später zu simulierenden Bauteil zu orientieren.

Neben dem Herstellungsprozess spielen auch die Belastungsarten und Randbedingungen für die Stoffgesetzanpassung eine entscheidende Rolle. Bei der Identifikation anhand von Standardprobekörpern werden deshalb die verwendeten Versuche entsprechend Bild 6.3 in Kap. 6.6.3 aufeinander abgestimmt. Zur Verdeutlichung wird wieder das I1-I2-Diagramm auf Basis der Hauptstreckungen herangezogen, wobei zusätzlich die Beanspruchung bei Annahme der maximalen Amplituden jeder Stufe gekennzeichnet sind (Bild 6.40 a). Da die Anpassung anhand der quasistationären Zyklen bis zu den eingezeichneten Punkten erfolgt, stellt der identifizierte Parametersatz unabhängig vom gewählten Stoffgesetz einen Kompromiss zwischen den vier Belastungsarten dar. Für die Simulation am Bauteil bedeutet dies, dass vor allem Beanspruchungsszenarien innerhalb desjenigen Gebietes, welches durch die Standardversuche abgedeckt wird, im Rahmen der Simulation wirklichkeitsgetreu dargestellt werden.

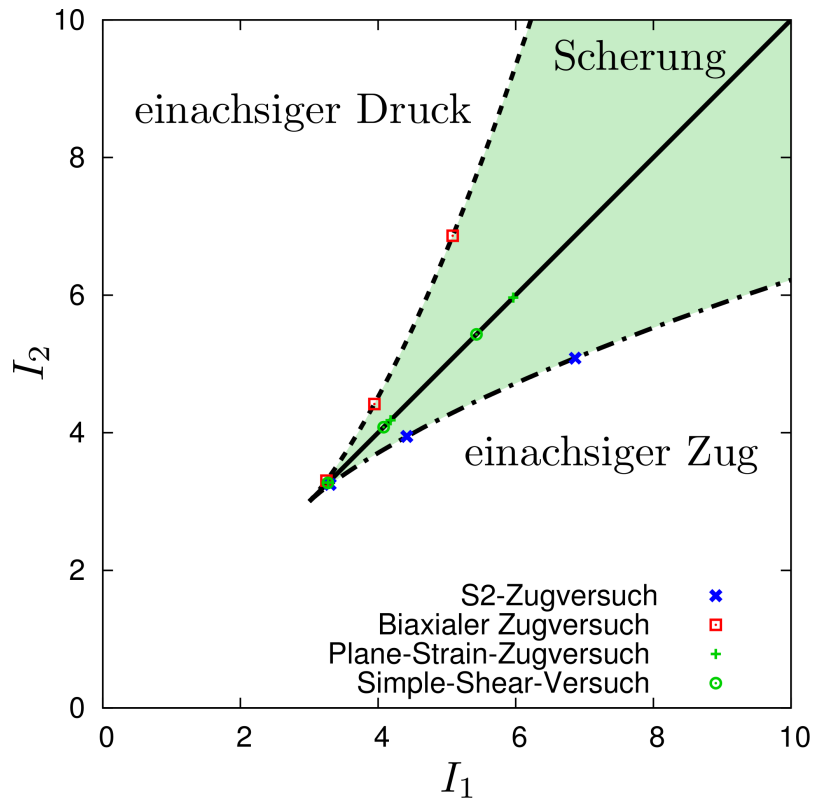

(a) für die maximalen Amplituden der mehrstufigen Versuche.

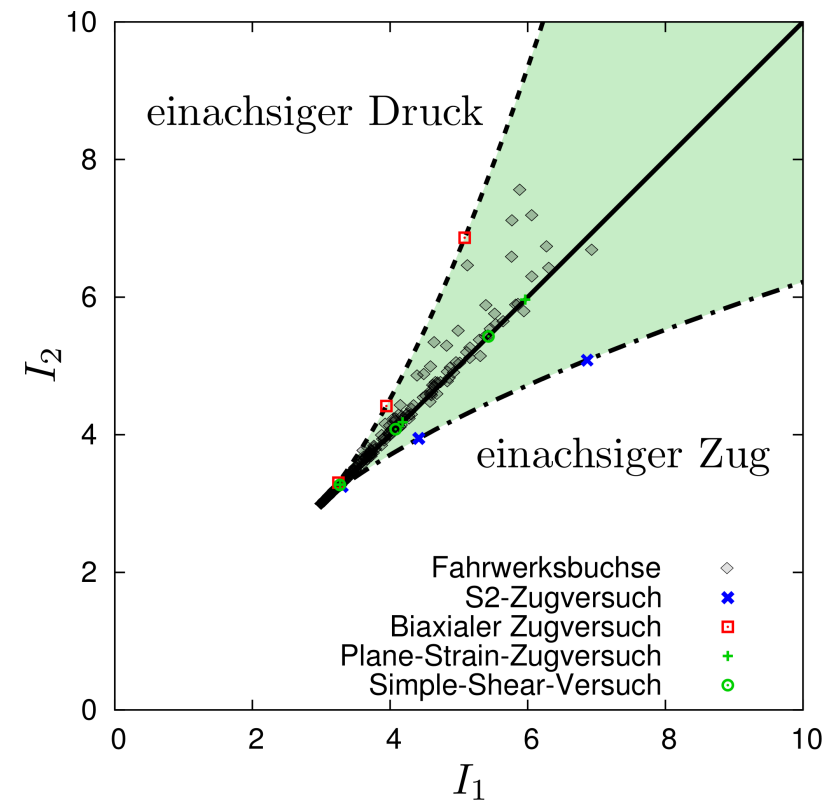

(b) für die Materialpunkte in der Gummischicht (Meridianschnitt) nach Kalibrieren und Abkühlen.

Bild 6.40: I1-I2-Diagramm mit Kennzeichnung der ersten und zweiten Hauptinvarianten. 
In Bild 6.40 b ist der Zustand innerhalb der Gummischicht der Fahrwerksbuchse nach dem Abkühlen und Kalibrieren dargestellt. Es sei darauf hingewiesen, dass lediglich alle Materialpunkte eines Meridianschnitts abgebildet sind, da sowohl Abkühlung als auch Kalibrierung axialsymmetrisch sind. Der überwiegende Teil der Materialpunkte befindet sich bezüglich der Hauptinvarianten innerhalb des durch die homogenen Versuche abgedeckten Gebietes, wobei sich der Schwerpunkt im Anfangsbereich des durch die Invarianten aufgespannten Trichters befindet.

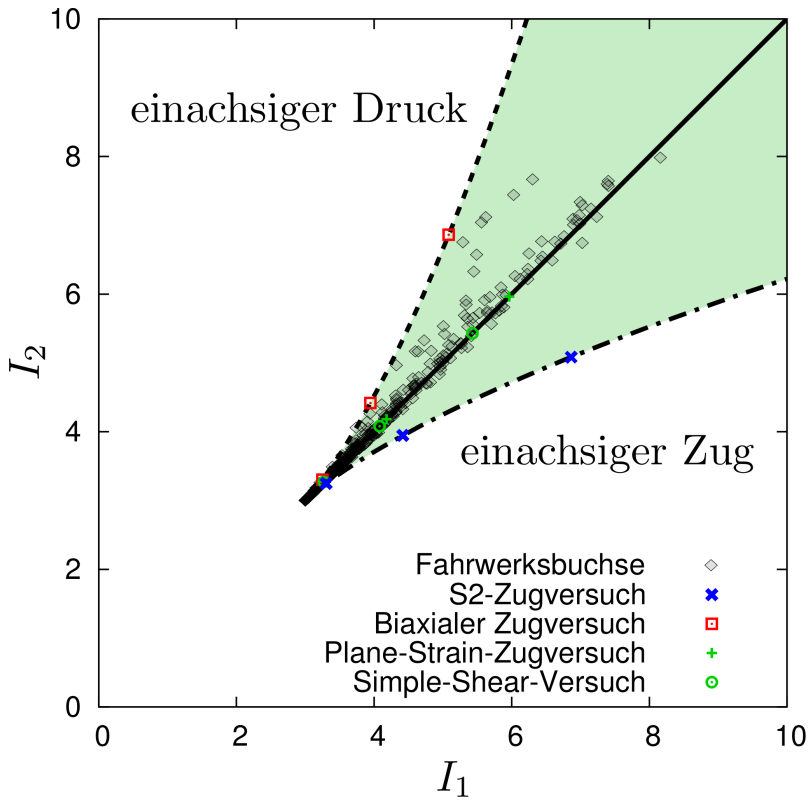

(a) axiale Belastung (Meridianschnitt) mit $1 \mathrm{KN}$.

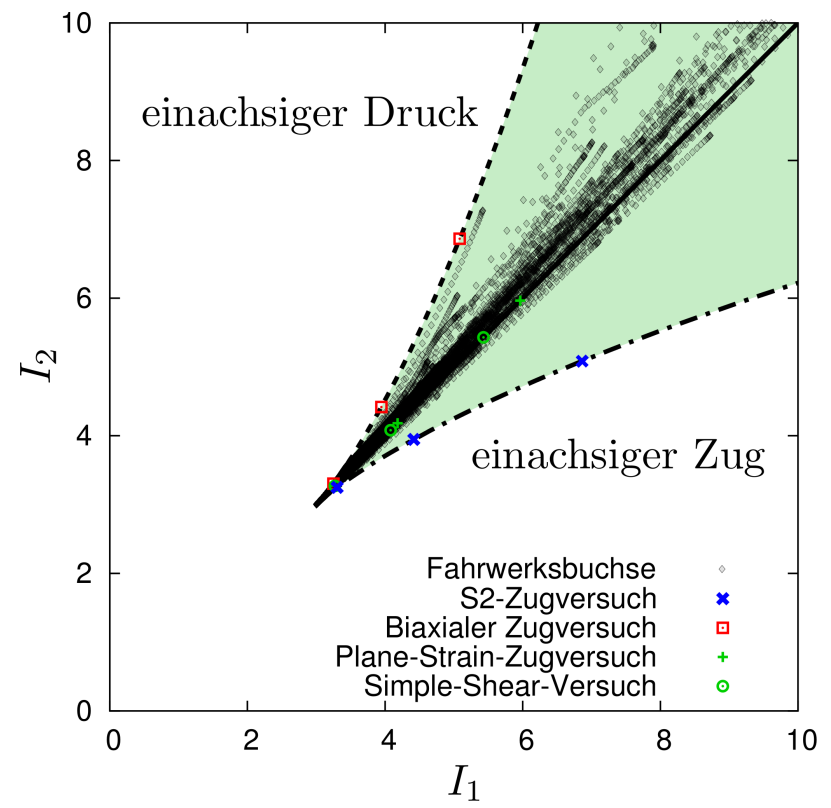

(c) radiale Belastung (Viertelmodell) mit $10 \mathrm{KN}$.

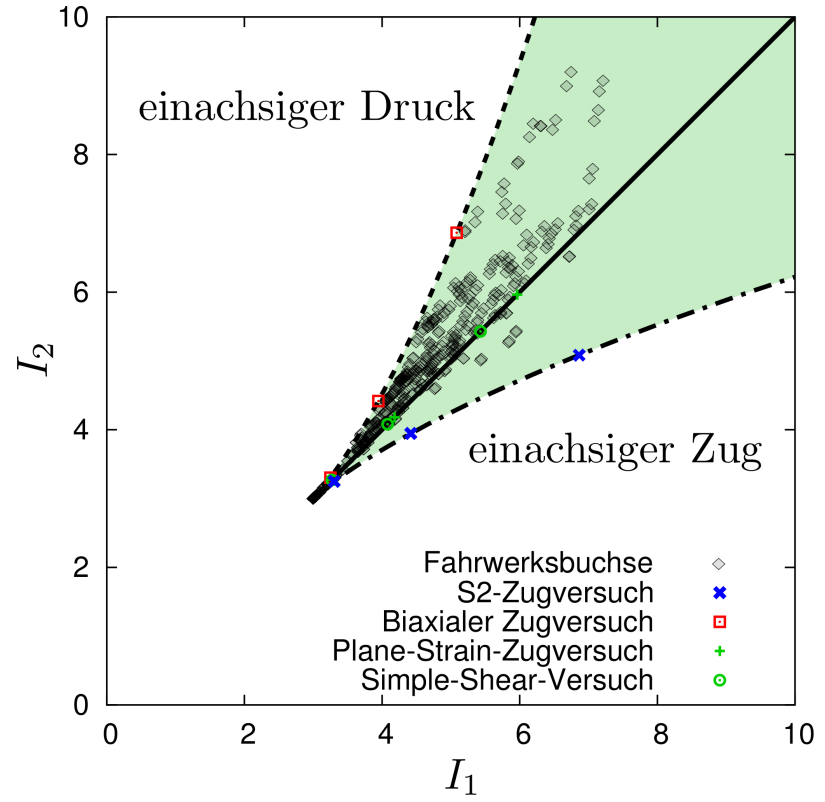

(b) Torsion (halber Meridianschnitt) mit $15^{\circ}$.

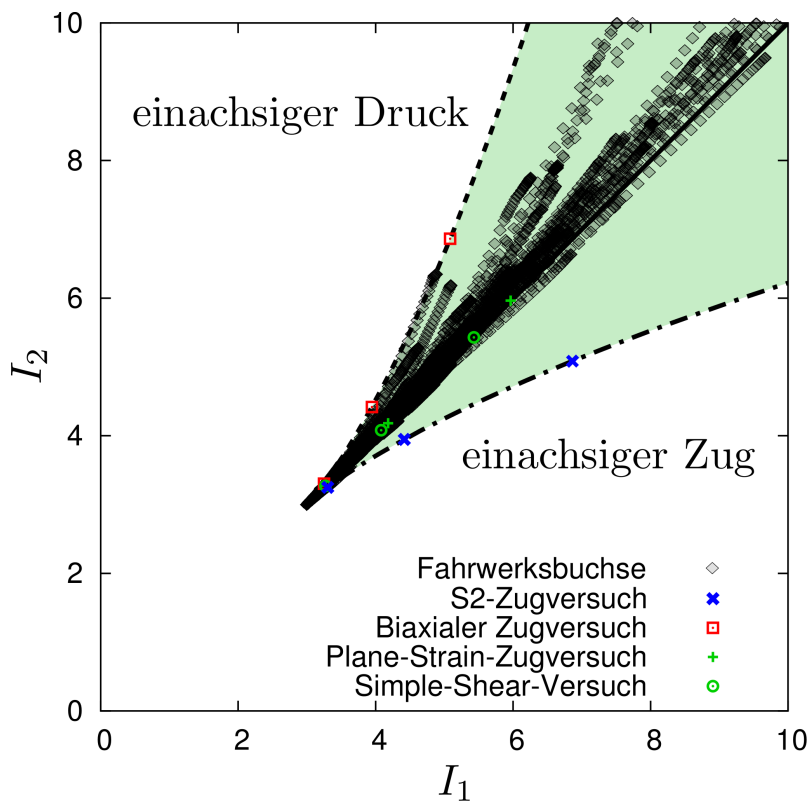

(d) kardanische Beanspruchung (Halbmodell) mit $3^{\circ}$.

Bild 6.41: I1-I2-Diagramm mit Kennzeichnung der ersten und zweiten Hauptinvarianten aller Materialpunkte in der Gummischicht.

Werden nun die im Lastenheft definierten Belastungen aufgebracht, ändert sich dies sehr deutlich (Bild 6.41). Während bei der axialen Beanspruchung der Unterschied zum kali- 
brierten Zustand noch moderat ausfällt, befindet sich bei Torsionsbeanspruchung bereits eine ganze Reihe Materialpunkte oberhalb des durch die Standardversuche abgedeckten Bereiches. Zudem bewegt sich der Beanspruchungsschwerpunkt deutlich in Richtung der Trichteröffnung. Bei radialer und kardanischer Belastung erfolgt dies noch in weitaus größerem Maße.

Dass die Materialpunkte vorwiegend auf der Druckseite liegen, erscheint schlüssig, da durch das Kalibrieren der hydrostatische Zug in der Buchse überkompensiert wird und die Belastungen diesen Effekt in großen Bereichen zusätzlich verstärken (vergl. Bild 6.17- Bild 6.20). Auf der Entlastungsseite hingegen (z.B. auf der "Unterseite" bei radialer Belastung) müssen die durch den Kalibriervorgang erzeugten hydrostatischen Druckspannungen erst kompensiert werden, sodass nur ein Teil der entsprechenden Materialpunkte dem Zugbereich zuzuordnen ist.

Bei der Spannungsberechnung in Materialpunkten, deren Verzerrungszustand sich weit außerhalb des Bereiches der Standardversuche im I1-I2-Diagramm befinden, kann die Extrapolation des verwendeten Stoffgesetzes mit den identifizierten Parametern zu außerordentlich großen Fehlern führen. Dies gilt insbesondere bei der Verwendung hyperelastischer Stoffgesetze (vergl. Bild 6.31). Als valider Lösungsansatz erscheint die Substitution der Standardprobekörper durch Probekörper, an welchen sich die in der Bauteilsimulation aufgebrachten Belastungen bereits im Rahmen der Parameteridentifikation darstellen lassen.

\subsubsection{Der bauteilnahe Probekörper}

Infolge der Vernetzung der Gummischicht entsteht eine Vielzahl von Elementen, insbesondere für das 3D-Modell (vergl. Kap. 6.5). Erste Simulationen zeigten zudem, dass bereits während des Kalibriervorgangs Selbstkontakt zu erwarten ist. Diese Tatsache ist auch aus dem tatsächlichen Fertigungsprozess der Fahrzeugbuchsen bekannt.

Die Identifikation von Materialparametern erfordert eine Vielzahl von FEM-Simulationen, welche zudem auch für ungünstige Parameterkonstellationen stabil konvergieren müssen. Die Verwendung des eigentlichen Bauteils zur Identifikation ist deshalb zwar bezüglich der Anpassungsgenauigkeit der Materialparameter ideal, jedoch nicht realisierbar. Benötigt wird vielmehr ein sogenannter "bauteilnaher Probekörper", welcher das Verhalten des Bauteils gut approximiert, dabei jedoch eine einfache Geometrie besitzt und stabile FEM-Simulationen ermöglicht.

Für die vorliegende Fahrwerksbuchse wird festgelegt, dass die radialen Abmessungen der metallische Innen- und Außenhülse beibehalten werden. Zudem sollen die Volumen der Gummischicht von Bauteil und Probekörper näherungsweise übereinstimmen, um die Steifigkeit der originalen Buchse möglichst wenig zu verändern.

Den Ausgangspunkt für sämtliche Belastungsszenarien stellt die kalibrierte Buchse dar. Um ein gutes FE-Netz für die unterschiedlichen Belastungen zu erhalten, sollte die Randkontur der Gummischicht im Ausgangszustand derart geformt sein, dass sie nach dem Kalibrieren eine möglichst geringe Krümmung aufweist. Aufgrund der Tatsache, dass der Kalibriervorgang die thermische und chemische Schrumpfung überkompensiert, kann von einer konkaven Randkontur ausgegangen werden. Um die Fertigung des Prototypenwerkzeugs 
zu vereinfachen, wird für die Randkontur ein Kreisabschnitt angenommen. Zur Ermittlung des Radius wurde eine Formidentifikation durchgeführt, wobei als Zielform ein Rand ohne Krümmung nach dem Kalibiervorgang zugrunde gelegt wurde. Die Materialparameter und der Wärmeausdehnungskoeffizient wurden einer früheren Identifikation für eine Buchse mit vergleichbarer Geometrie und Härte entnommen. Auf dieser Basis wurde ein Radius von $r_{\text {Rand }}=2,5 \mathrm{~mm}$ ermittelt (Bild 6.42) und zur Herstellung der Werkzeugmatrize verwendet.

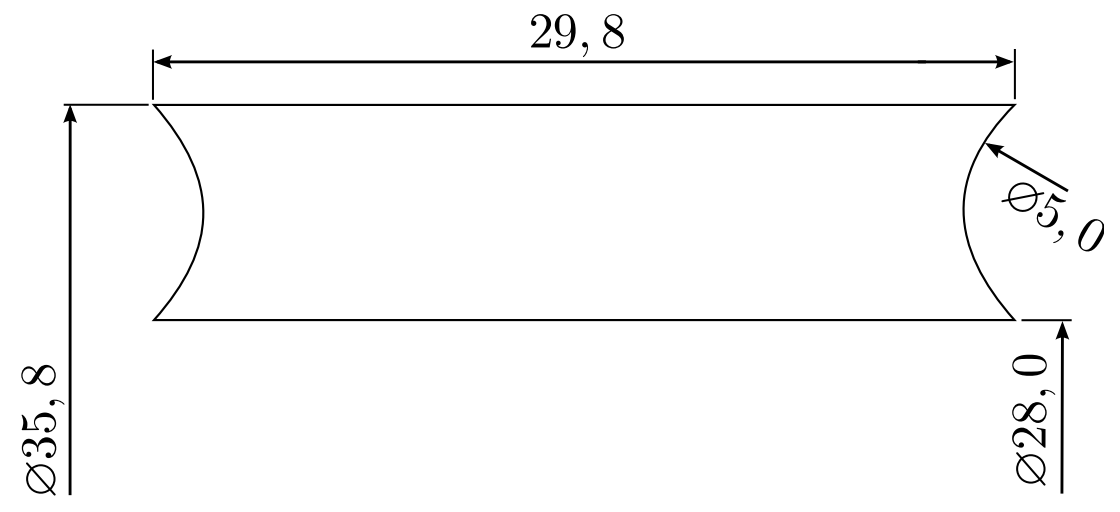

Bild 6.42: Meridianschnitt der Gummischicht des bauteilnahen Probekörpers.

Sowohl der Herstellungsprozess als auch der Kalibriervorgang sind für das Bauteil und den bauteilnahen Probekörper identisch. Bild 6.43 und Bild 6.44 stellen den bauteilnahen Probekörper im abgekühlten Zustand vor bzw. nach der Kalibrierung dar.

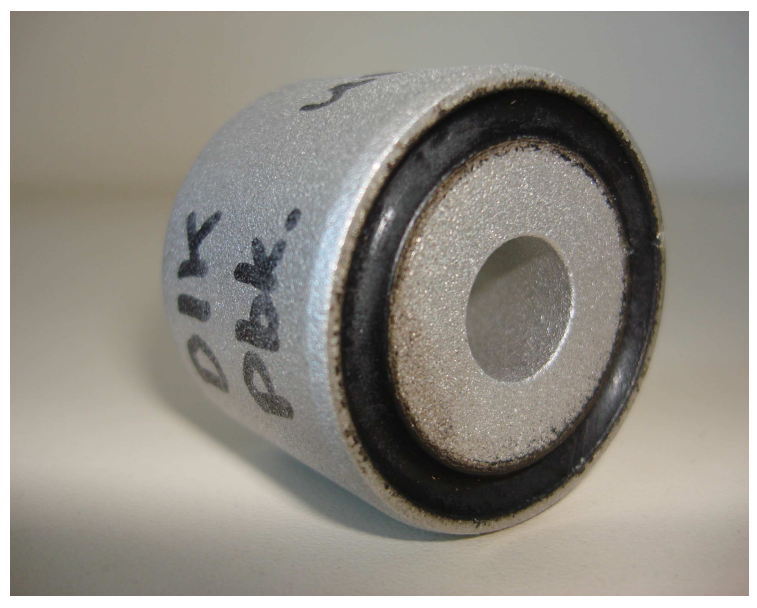

Bild 6.43: Probekörper nach dem Abkühlen.

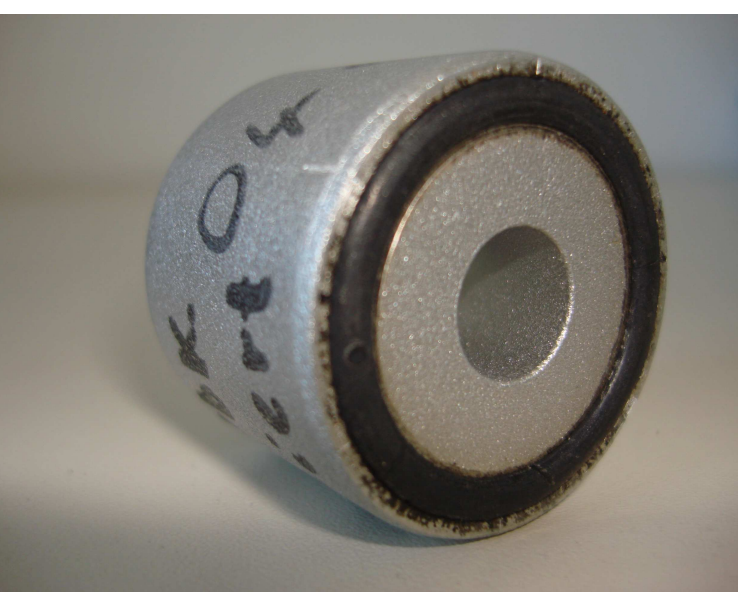

Bild 6.44: Probekörper nach dem Kalibrieren.

Die Vermessung des bauteilnahen Probekörpers nach dem Abkühlen und Kalibrieren zeigt deutliche Unterschiede gegenüber der Simulation. Bei der Festlegung der Probekörpergeometrie war aufgrund der Erfahrungen mit vergleichbaren Gummimischungen ein Wärmeausdehnungskoeffizient von $\alpha_{t}=0,00014 \mathrm{~K}^{-1}$ angenommen worden. Die Volumenverringerung infolge des Abkühlens um 150K ist in der Simulation deutlich größer als bei der Messung des thermischen und chemischen Schrumpfes des bauteilnahen Probekörpers. Die Auswertung der Volumenverringerung beim Kalibrieren infolge der Kompression der Gummischicht zeigt zudem, dass diese in der Simulation 0,63\%, bei der Messung jedoch 1,75\% beträgt. 
Möglicherweise ist der in der Simulation angenommene Kompressionsmodul $K=5000 \mathrm{MPa}$ zu hoch gewählt.

Der tatsächliche Zustand nach dem Abkühlen spielt für die FEM-Simulation nur eine untergeordnete Rolle. Vielmehr ist der Zustand nach dem Kalibrieren entscheidend, da dieser die Basis der verschiedenen Beanspruchungen darstellt. Dementsprechend wird die Übereinstimmung von Messung und Simulation zu diesem Zeitpunkt angestrebt. Dabei wird der Kompressionsmodul als konstant angenommen und lediglich der Wärmeausdehnungskoeffizient identifiziert. Die Lösung des Formidentifikationsproblems durch die Anpassung der Simulation die Messergebnisse nach dem Kalibriervorgang führt auf $\alpha_{t}=9,87 \cdot 10^{-5} \mathrm{~K}^{-1}$ und wird für alle folgenden FEM-Simulationen verwendet.

\subsubsection{Simulation der Lastfälle am bauteilnahen Probekörper}

Um möglichst zuverlässige Materialparameter für die FEM-Simulationen des Originalbauteils zu erhalten, entsprechen die zur Identifikation verwendeten Experimente den Belastungen im Lastenheft. Demnach ist die Nutzung eines energetischen oder geometrischen Vergleichsmaßes zur Abstimmung der verschiedenen Belastungen aufeinander von vornherein nicht möglich. Dies stellt jedoch kein Problem dar, da auch hier die Verwendung der GFK als Zielfunktionswert eine gute Wahl darstellt. Bei der Vernetzung soll sichergestellt werden, dass für alle Belastungen die Topologie der FE-Netze nicht voneinander abweicht. Entsprechende Testsimulationen zeigen jedoch, dass bei Nutzung einer regelmäßigen Netztopologie die FEM-Simulationen nicht für alle Beanspruchungen stabil konvergieren und die FE-Netze teilweise stark verzerrt werden. Die Netztopologie wurde daher gezielt modifiziert und ist in Bild 6.45 dargestellt.

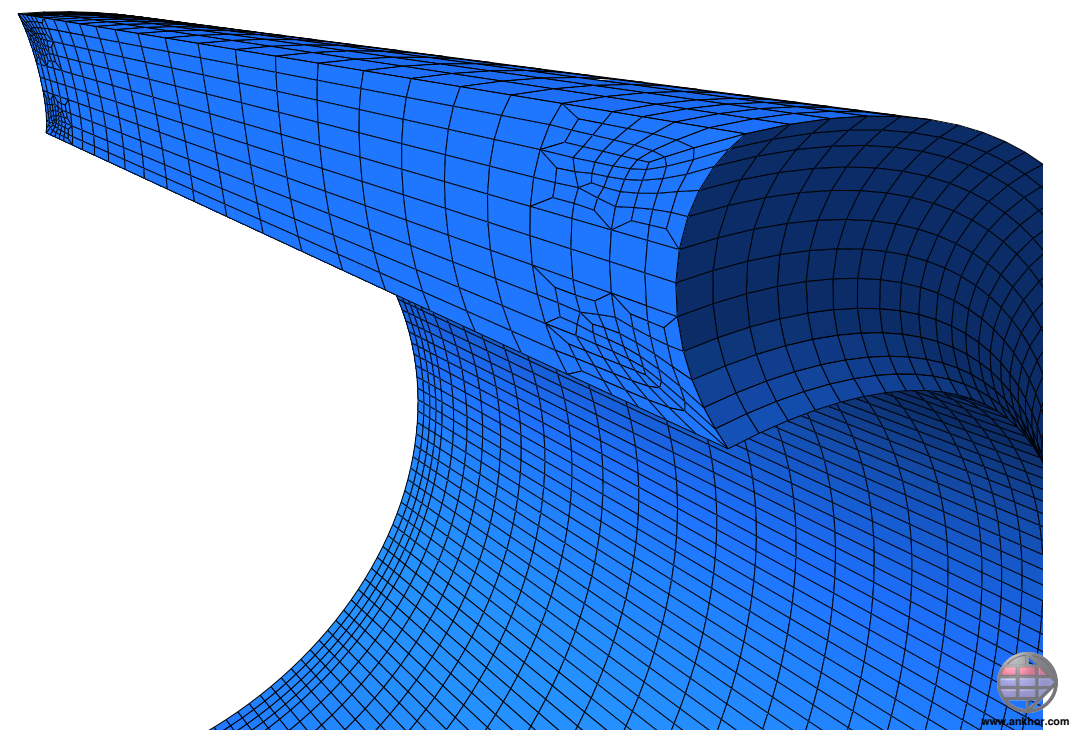

Bild 6.45: FE-Netz des undeformierten bauteilnahen Probekörpers.

In Bild 6.46 ist der deformierte Probekörper nach dem Abkühlen um $150 \mathrm{~K}$ dargestellt. Der anschließende Kalibriervorgang führt zu den in Bild 6.47 dargestellten Vergleichsdehnungen. Obwohl die verschiedenen Belastungen noch nicht aufgebracht wurden, sind einige Bereiche des Probekörpers bereits stark deformiert. 


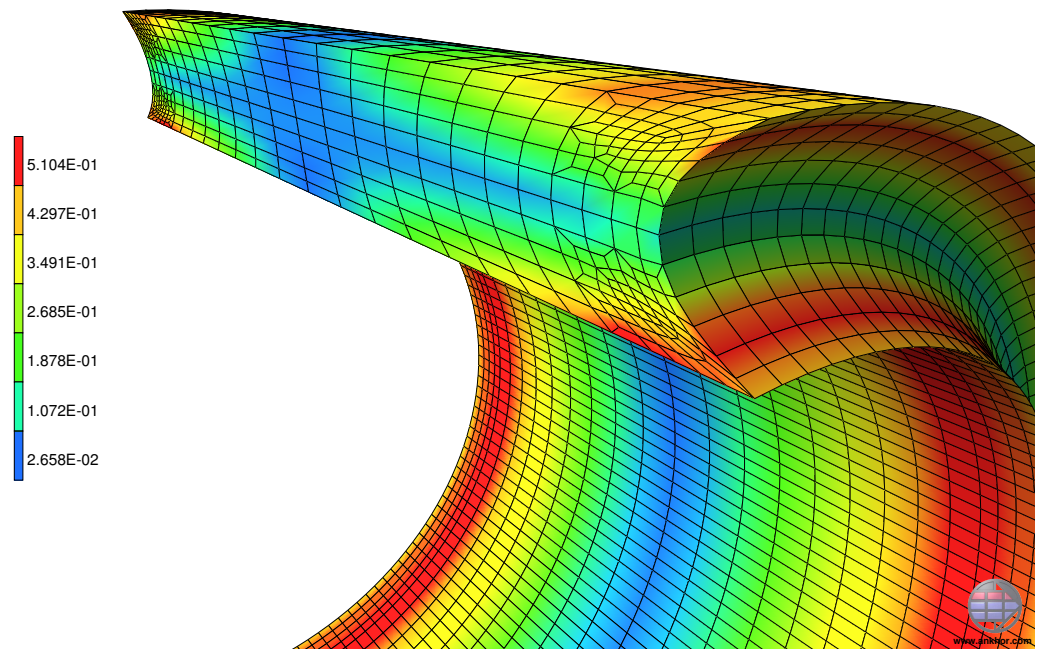

Bild 6.46: Vergleichsdehnungen im bauteilnahen Probekörper nach dem Abkühlen.

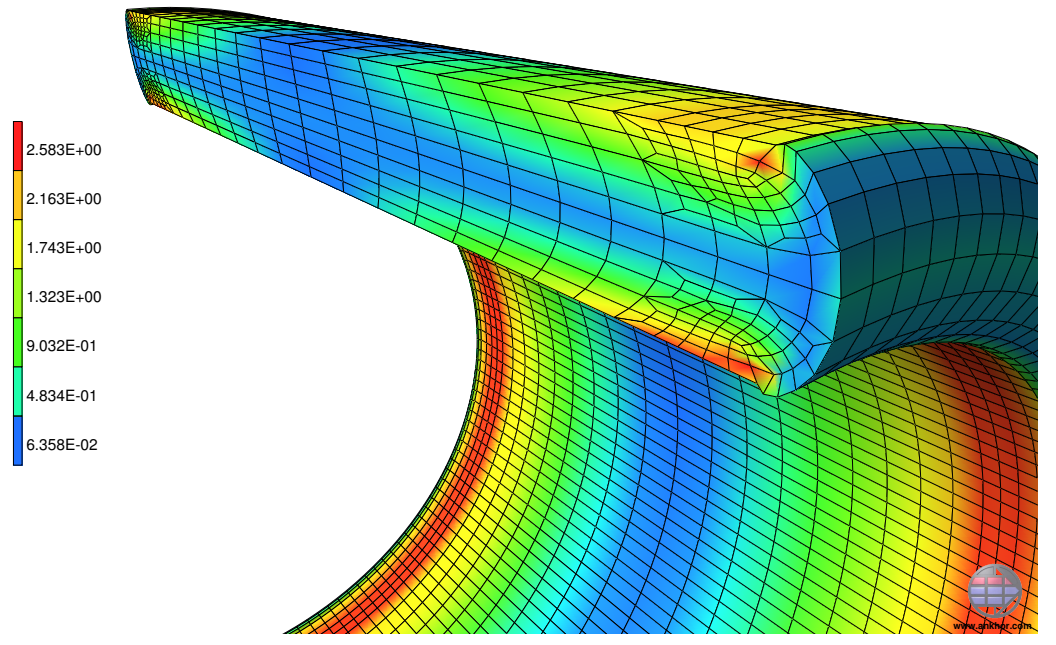

Bild 6.47: Vergleichsdehnungen im bauteilnahen Probekörper nach dem Kalibrieren.

Interessant ist an dieser Stelle die Auswertung der hydrostatischen Spannungen in der Gummischicht nach dem Kalibriervorgang. Diese sind für das Bauteil in Bild 6.48 a sowie für den bauteilnahen Probekörper in Bild 6.48 b dargestellt.

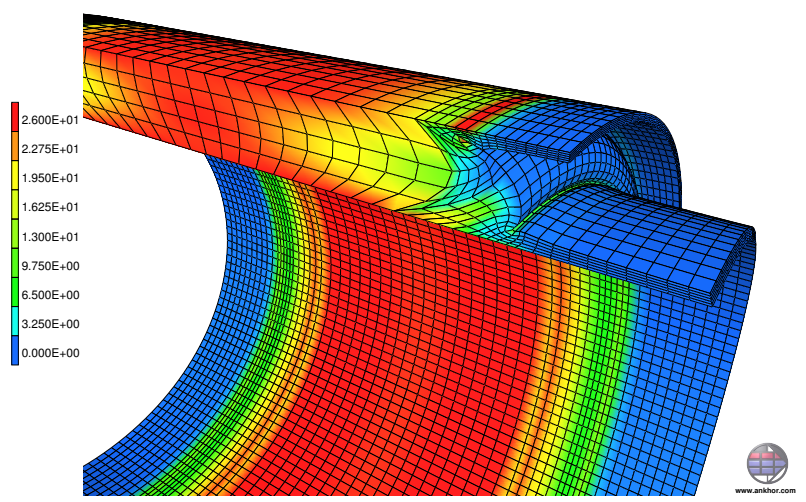

(a) für das Bauteil.

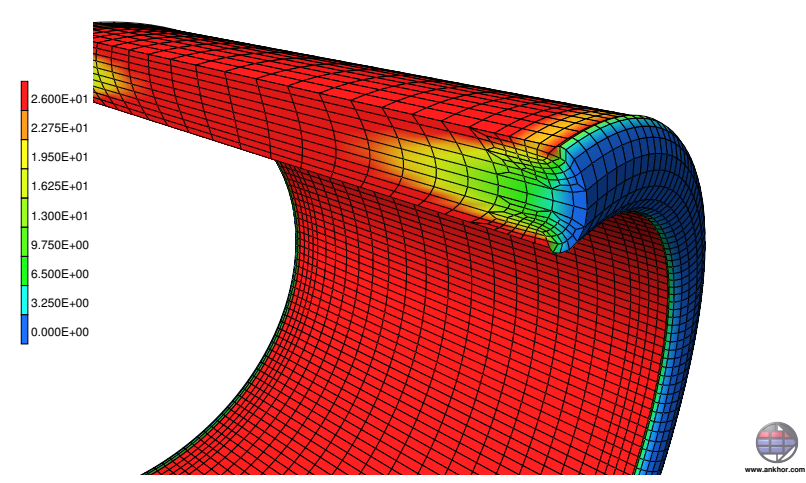

(b) für den bauteilnahen Probekörper.

Bild 6.48: Hydrostatischer Spannungsanteil nach dem Abkühlen und Kalibrieren. 
Anhand der Abbildungen ist gut sichtbar, dass das Kalibrieren der Fahrwerksbuchse bzw. des zugehörigen Probekörpers in beiden Fällen die nach dem Abkühlen vorhandenen hydrostatischen Zugspannungen nicht nur kompensiert, sondern zu beträchtlichem hydrostatischen Druck führt. Unter der Annahme, dass eine Fahrwerksbuchse in der Praxis tendenziell erst nach mehreren Tagen oder Wochen im Fahrzeug verbaut wird, ist der Abbau eines Teils dieser hydrostatischen Druckspannungen infolge von Relaxation entsprechend Kap. 6.4 sehr wahrscheinlich. Da Relaxation jedoch mit den hier betrachteten Stoffgesetzen nicht darstellbar ist, wird dies weder bei der Identifikation, noch bei der eigentlichen Simulation berücksichtigt.

Im Anschluss an die Kalibrierung werden analog zum Bauteil die Belastungen aus dem Lastenheft aufgebracht. Im Folgenden werden die entsprechenden FEM-Simulationen am bauteilnahen Probekörper betrachtet.

\section{Axiale Belastung}

Analog zum Bauteil genügt für die entsprechende Simulation ein rotationssymmetrisches FEModell. Aufgrund des dadurch bedingten geringen Rechenaufwandes soll anhand dessen die Simulation des Abkühl- und Kalibriervorgangs für unterschiedliche Vernetzungsdichten untersucht werden. Während für die grobe Vernetzung nach dem Kalibrieren eine rein konvexe Randkontur vorliegt, werden bei feiner werdender Vernetzung in der Randkontur Bereiche mit konkaver Form sichtbar (Bild 6.49 a). Bereits während der Kalibrierung kommt es zum kompletten Einklappen der Randkontur (Bild 6.49 b) und anschließend zu Selbstkontakt.

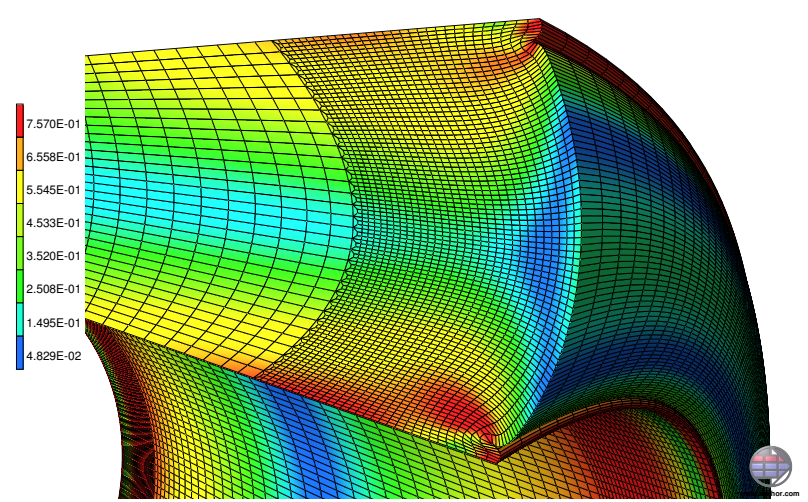

(a) Nach dem Kalibrieren

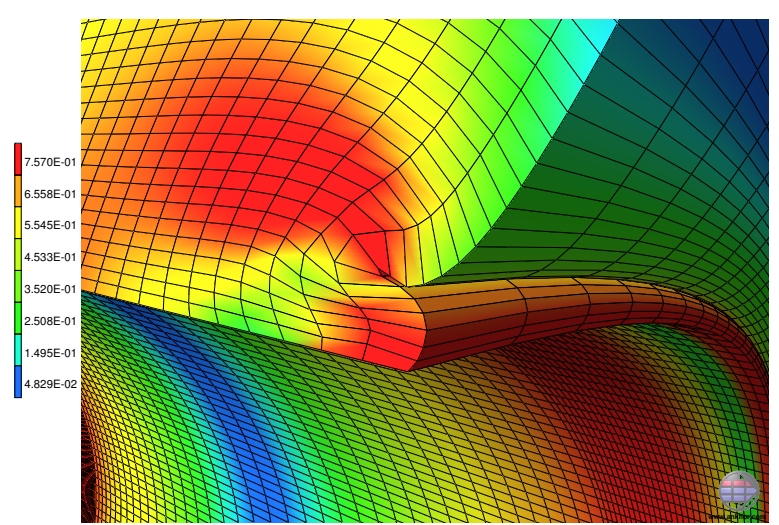

(b) Unmittelbar vor Selbstkontakt

Bild 6.49: Vergleichsdehnungen im Probekörper mit extrem feiner Vernetzung.

Da in den für die Identifikation verwendeten Simulationen aus Rechenzeitgründen auf Kontakt verzichtet werden soll und das Einklappen die Konvergenz der Simulationen stark beeinträchtigt, ist die Prüfung der Auswirkungen des lokalen Einklappens auf die globalen Reaktionsgrößen notwendig. Zu diesem Zweck werden die Kraft-Verschiebungs-Kurven für die axiale Belastung bei grober und feiner Vernetzung miteinander verglichen (Bild 6.50). Dabei ist zu beobachten, dass das lokale Einklappen nahezu keinen Einfluss auf die globale Reaktionskraft hat. Es ist somit zulässig, die grobe Vernetzung zu nutzen, auch wenn dabei dieses lokale Verhalten nicht berücksichtigt wird. Zudem war in den tatsächlichen Versuchen dieses Einklappen nicht zu beobachten, wobei der Grund für den Unterschied zwischen Ex- 
periment und Simulation mit sehr feiner Vernetzung noch genauer betrachtet werden sollte.

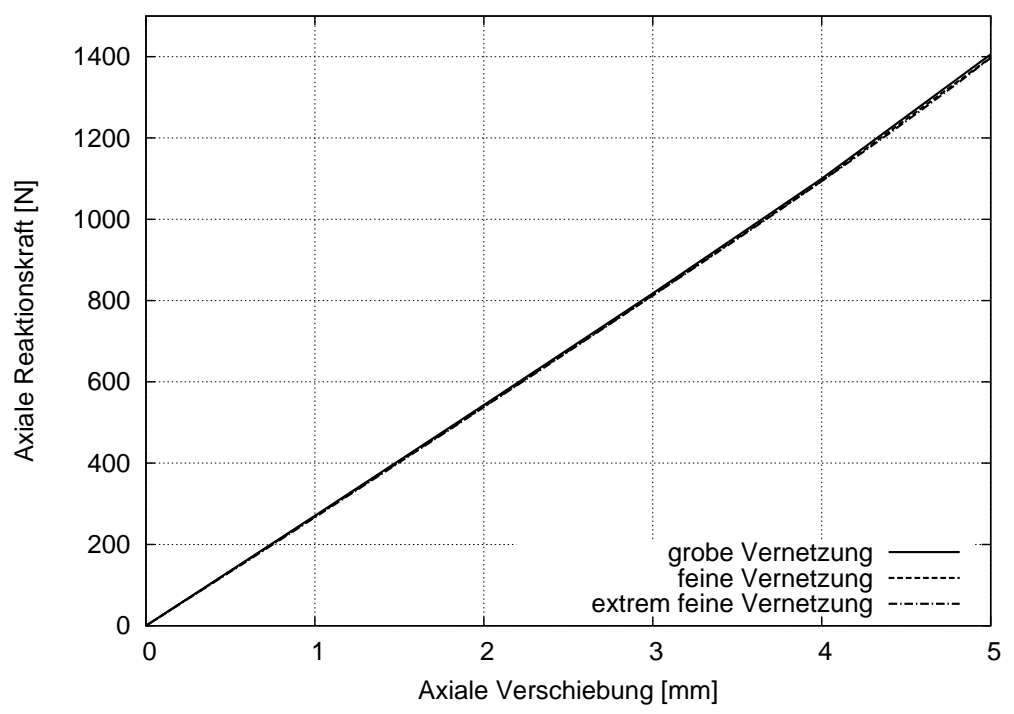

Bild 6.50: Vergleich der Kraft-Verschiebungs-Kurven bei axialer Belastung für unterschiedliche Vernetzungen.

Ein weiterer interessanter Aspekt ist der Vergleich der Simulation bei Variation des Kompressionsmoduls. Allgemein wird für Gummiwerkstoffe von quasi inkompressiblem Materialverhalten ausgegangen. Um dies auf FEM-Simulationen übertragen zu können, wird ein möglichst hoher Kompressionsmodul $K$ angenommen. Allerdings verschlechtert sich das Konvergenzverhalten der Simulation bei steigendem Kompressionsmodul zunehmend. Sowohl bei der Homogen-Identifikation als auch bei der Identifikation anhand bauteilnaher Probekörper wird zunächst $K=5000 \mathrm{MPa}$ angenommen. Da die Konvergenz der Simulationen für die Belastung der eigentlichen Fahrwerksbuchse bei Nutzung eines derart hohen $K$ jedoch teilweise schlecht ist, erfolgt zu Testzwecken die Simulation der axialen Belastung des bauteilnahen Probekörpers für unterschiedliche $K$. Dabei wird sichtbar, dass die Verringerung auf $K=500 \mathrm{MPa}$ bzw. $K=50 \mathrm{MPa}$ zu einem drastisch anderen Deformationszustand führt (Bild 6.51 - Bild 6.531).

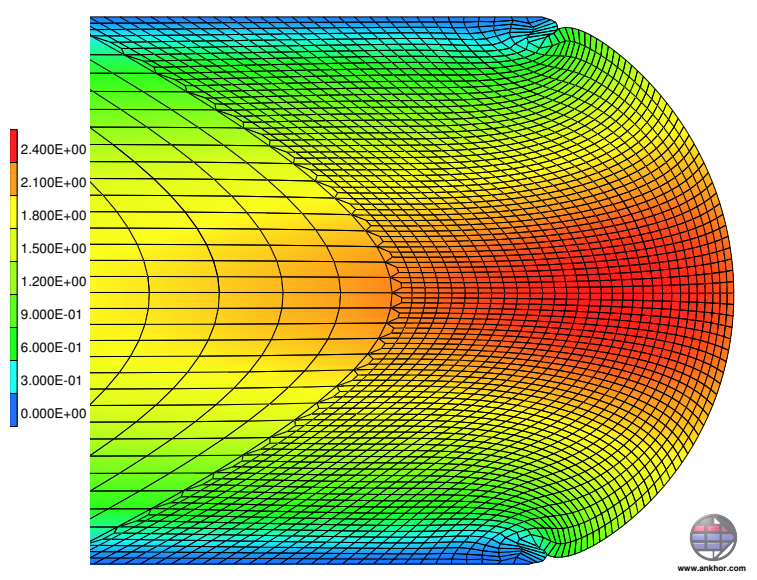

Bild 6.51: Axiale Verschiebung für $K=5000 \mathrm{MPa}$.

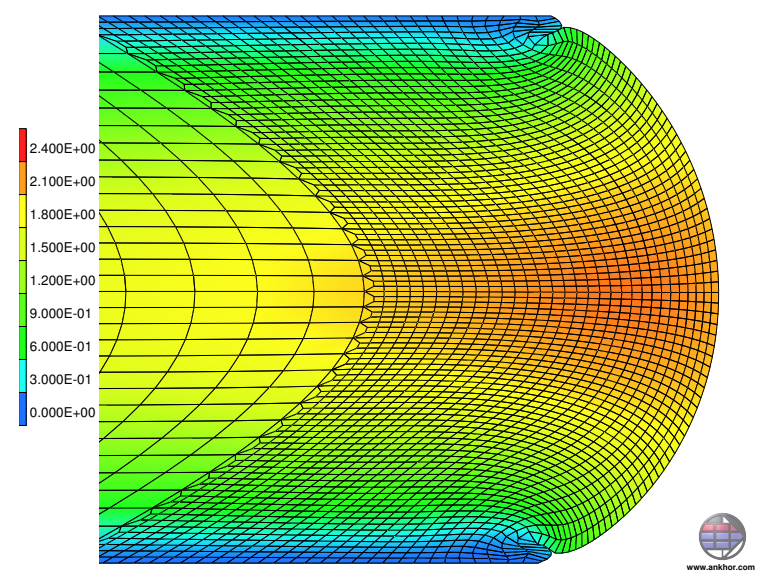

Bild 6.52: Axiale Verschiebung für $K=500 \mathrm{MPa}$. 


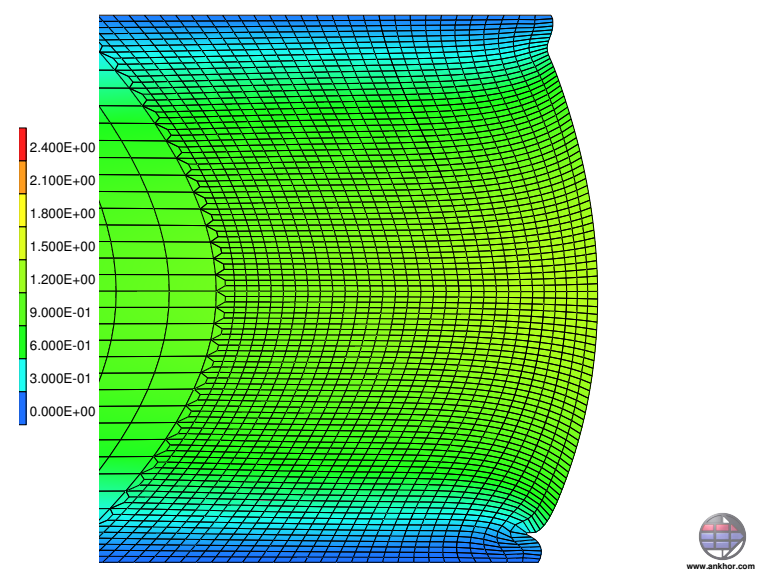

Bild 6.53: Axiale Verschiebung für $K=50 \mathrm{MPa}$.

Bei zusätzlicher Berücksichtigung der deutlichen Unterschiede zwischen Experiment und entsprechender FEM-Simulation des Abkühlens bzw. des Kalibrierens liegt der Schluss nahe, dass die Annahme eines sehr hohen, temperatur- und belastungsunabhängigen Kompressionsmoduls fragwürdig ist. Dies erscheint auch deshalb schlüssig, da die Abhängigkeit des Kompressionsmoduls vom Umgebungsdruck bereits länger bekannt ist (z.B. Adams und Gibson (1930)). Der beim Kalibriervorgang entstehende hohe hydrostatische Druck innerhalb der Gummischicht der Fahrwerksbuchse hat demnach potentiell einen deutlichen Einfluss auf den Kompressionsmodul, was auch Stommel und Zimmermann (2012) darlegen. Da darüber hinaus auch die verschiedenen Bauteilbelastungen Einfluss auf den hydrostatischen Druck haben und diese Problematik unabhängig vom verwendeten Stoffgesetz ist, erscheint eine detaillierte Untersuchung im Rahmen eines eigenständigen Forschungsprojektes sehr sinnvoll.

Die Simulation der axialen Belastung unter Verwendung der groben Vernetzung mit $K=5000 \mathrm{MPa}$ für die Maximalbelastung von $F^{\text {axial }}=1 \mathrm{KN}$ führt auf die in Bild 6.54 dargestellten Vergleichsdehnungen.

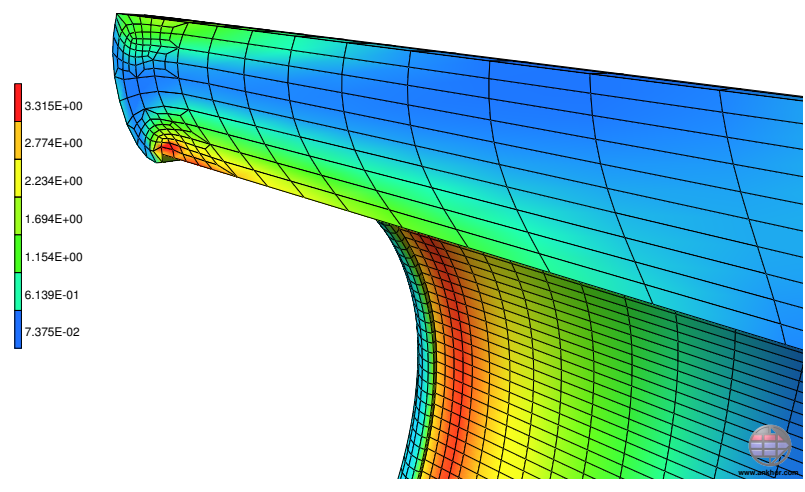

(a) Rückseite

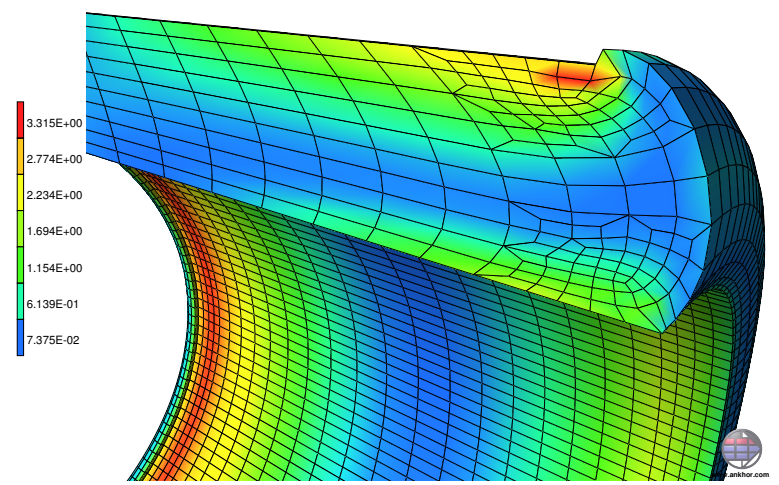

(b) Vorderseite

Bild 6.54: Vergleichsdehnungen bei axialer Belastung (1 KN). 


\section{Radiale Belastung}

Die radiale Belastung des bauteilnahen Probekörpers ist ebenso wie beim Bauteil nicht rotationssymmetrisch. Somit wird auch hierfür ein 3D-Netz unter Ausnutzung der Symmetrieebenen in axialer Richtung sowie der Symmetrieebene, welche den mittigen Meridianschnitt darstellt, für die FEM-Simulation verwendet. Aus der radialen Maximalbelastung von $F^{r a d}=10 \mathrm{KN}$ resultieren die in Bild 6.55 dargestellten Vergleichsdehnungen. Dabei treten in einigen Elementen sehr ungünstige Innenwinkel auf, welche zwar lokal zu Fehlern führen, auf die globalen Reaktionsgrößen jedoch keinen signifikanten Einfluss haben.

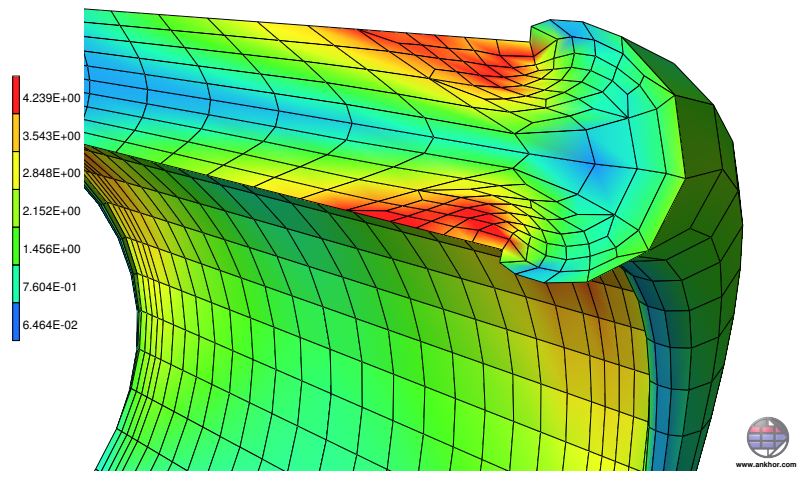

(a) Oberseite

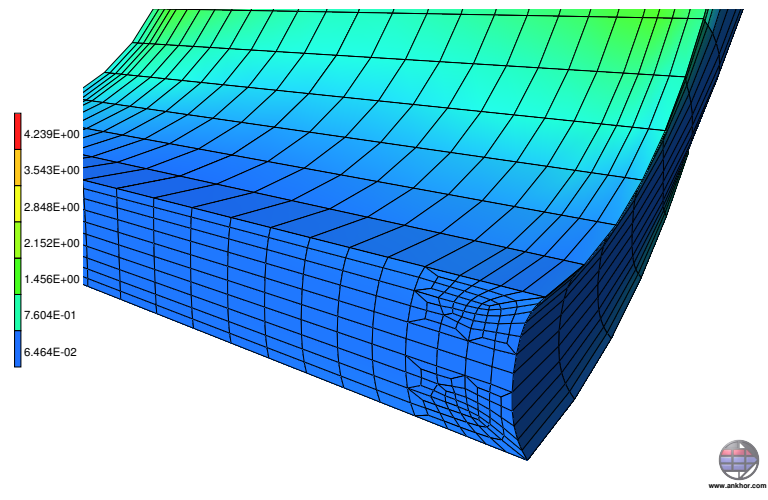

(b) Unterseite

Bild 6.55: Vergleichsdehnungen bei radialer Belastung (10 KN).

\section{Kardanische Belastung}

Die kardanische Belastung wird bewusst nicht zur Identifikation verwendet. Einerseits ist der Rechenaufwand aufgrund des analog zum Originalbauteil notwendigen Halbmodells vergleichsweise groß, was zu einer erheblichen Erhöhung des Rechenaufwandes im Rahmen der Identifikation führen würde. Darüber hinaus soll diese Belastung zur Validierung der ermittelten Materialparameter genutzt werden. Dies ist vor allem deshalb sinnvoll, weil sich die infolge der kardanischen Beanspruchung einstellenden Verzerrungen in der Gummischicht außerordentlich stark von denen der übrigen Belastungen unterscheiden.

\section{Torsionsbelastung}

Auch die Torsionsbeanspruchung des bauteilnahen Probekörpers orientiert sich an der Belastung des Bauteils. Entsprechend Kap. 6.5 wird auch hier nicht der durch MSC.MARC bereitgestellte Elementtyp "Twist \& Herrmann Formulation", sondern ein Schichtmodell genutzt. Die Simulation für die Maximalverdrehung von $\varphi^{\text {Tors }}=15^{\circ}$ führt auf die in Bild 6.56 dargestellten Vergleichsdehnungen. 


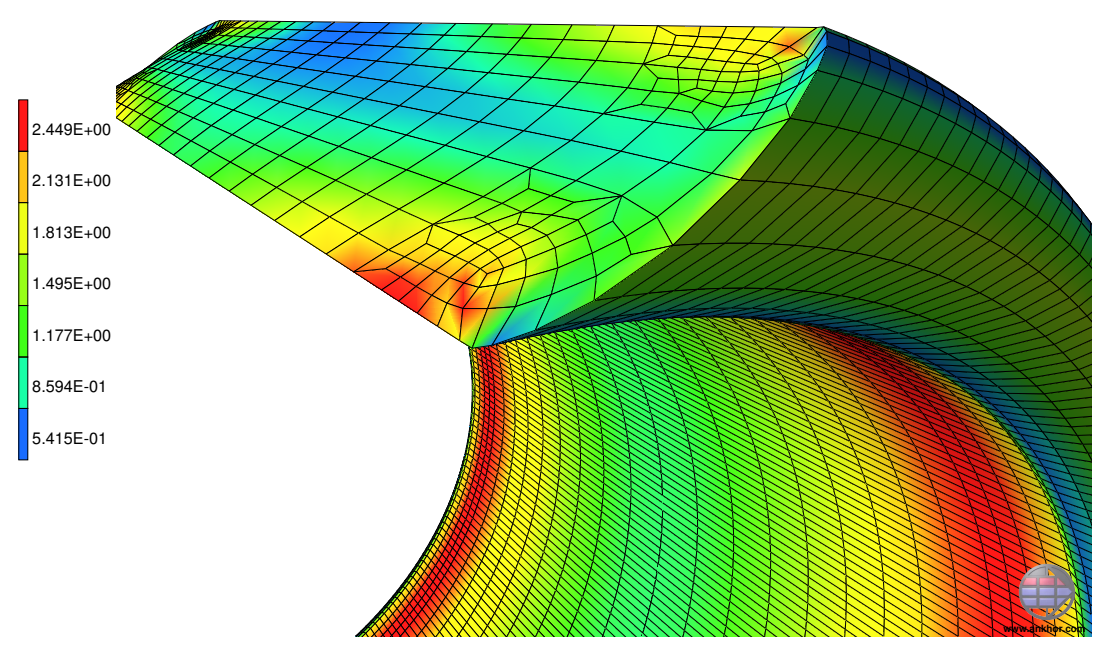

Bild 6.56: Vergleichsdehnungen bei Torsionsbeanspruchung $\left(15^{\circ}\right)$.

\subsubsection{Inhomogen-Parameteridentifikation}

Aufgrund der in Kap. 6.6.4 beschriebenen Korrelation zwischen den Basen und Exponenten der OGDEn-Terme wird das OGDEN-Stoffgesetz an dieser Stelle nicht berücksichtigt und die Identifikation anhand bauteilnaher Probekörper ausschließlich für YEOH und MORPH in RepRicht durchgeführt.

Um die Identifikation anhand bauteilnaher Probekörper durchführen zu können, wurden für die verschiedenen Lastfälle entsprechende Versuche am bauteilnahen Probekörper durchgeführt. Für die Identifikation der YEOH-Parameter wurden die Versuche ebenso wie bei den Standardprobekörpern einstufig durchgeführt und der näherungsweise stationäre sechste Zyklus zur Anpassung verwendet. Beispielhaft ist dafür die Kraft-Verschiebungs-Kurve der axialen Belastung in Bild 6.57 dargestellt.

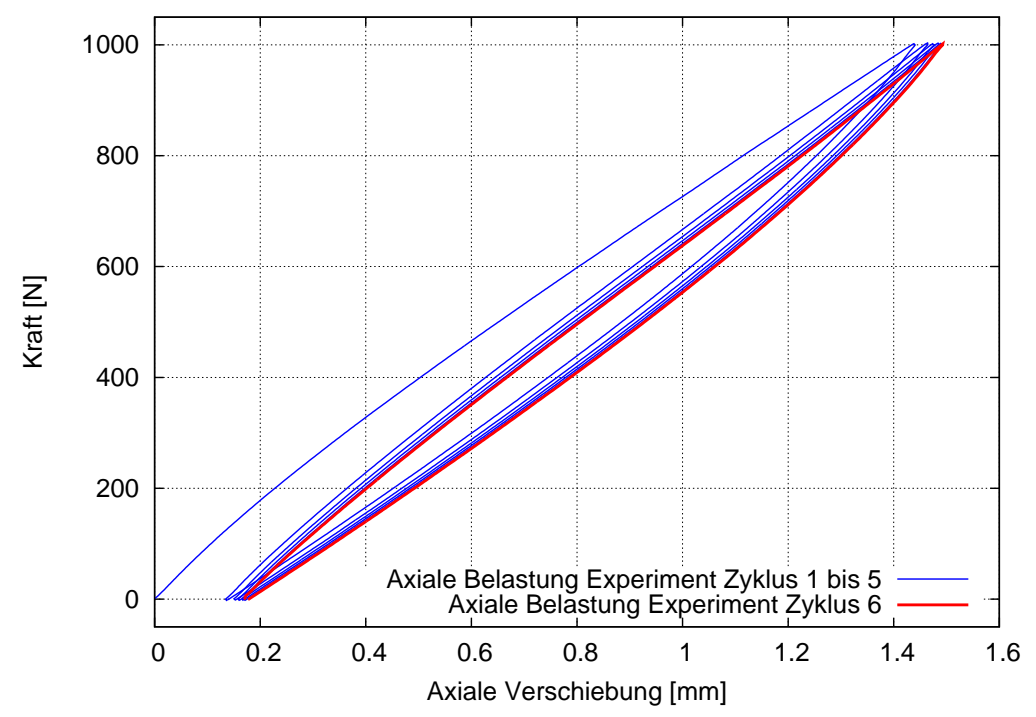

Bild 6.57: Kraft-Verschiebungs-Kurve für einstufige axiale Belastung ( $1 \mathrm{KN})$ des bauteilnahen Probekörpers. 
Für das MoRPH-Stoffgesetz hingegen sind analog zur Homogen-Identifikation mehrstufige Versuche notwendig, um genügend Informationen zur Identifikation der Parameter bereitzustellen. Da sich im Gegensatz zu den Standardprobekörpern bei der Fahrwerksbuchse einer Belastungsamplitude keine einzelne klar definierte Deformationsgröße zuordnen lässt, werden die drei Belastungsstufen für jeden Lastfall linear bezüglich der Maximalbelastung unterteilt und der jeweilige quasistätionäre Zyklus genutzt (Bild 6.58).

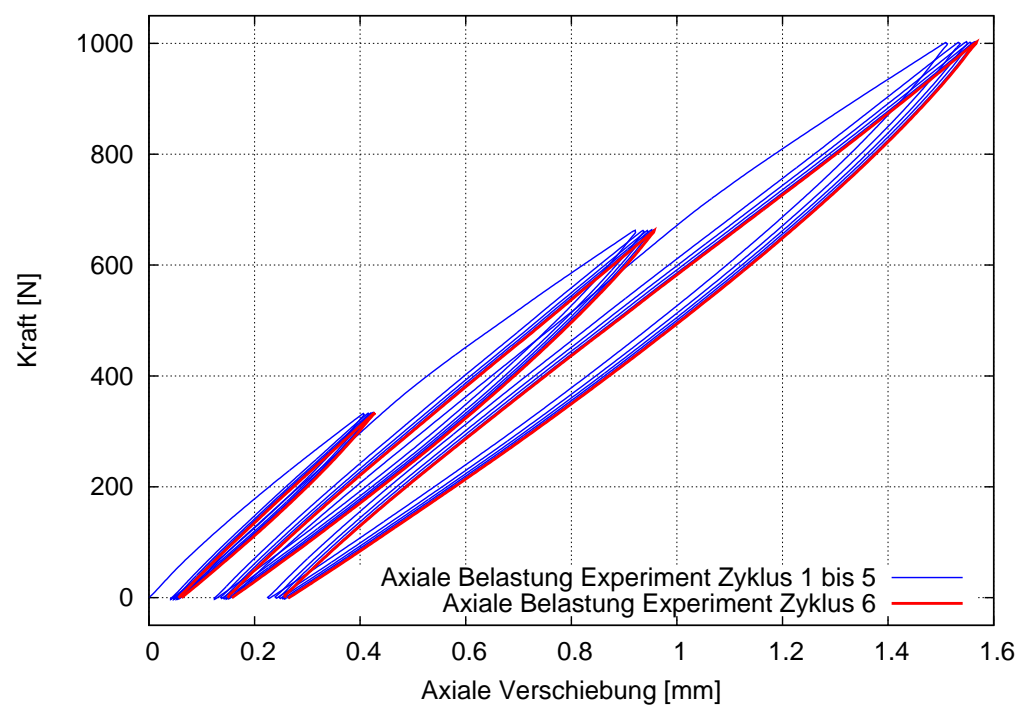

Bild 6.58: Kraft-Verschiebungs-Kurve für mehrstufige axiale Belastung des bauteilnahen Probekörpers.

Infolge der Art des Versuchsaufbaus erfolgt das Aufbringen der Belastungen für Kardanik und Torsion verschiebungsgesteuert in Form einer Winkeländerung, für die axiale und radiale Belastung jedoch kraftgesteuert. Wie in Kap. 6.6.1 dargelegt, ist die Simulation der kraftgesteuerten Belastung im Rahmen der Identifikation anhand bauteilnaher Probekörper bei Verwendung von MoRPH in RepRicht aufwändig und mit hohem Rechenaufwand verbunden. Die axiale und radiale Belastung wird deshalb trotz kraftgesteuerter Versuche in den entsprechenden Simulationen verschiebungsgesteuert aufgebracht.

Aus messtechnischen Gründen war es nicht möglich, die mehrstufigen Belastungen innerhalb eines einzelnen Experimentes durchzuführen. Vielmehr musste für jede Stufe nach Erreichen des quasistationären Zustandes das Experiment unterbrochen, die Versuchssteuerung an die nächste Belastungsamplitude angepasst und das Experiment neu gestartet werden. Im Rahmen der Aufbereitung der Messdaten müssen somit für jede Banspruchungsart die drei Teilexperimente derart dargestellt werden, dass die Anfangspunkte der zweiten und dritten Belastungsamplitude mit den Endpunkten der vorangegangenen Amplitude übereinstimmen. Bei Betrachtung von Bild 6.58 fällt jedoch auf, dass trotz dessen die Erstbelastung der zweiten und dritten Stufe nicht mit der Belastungskurve des jeweils vorangegangenen quasistätionären Zyklus übereinstimmt (im Gegensatz zum unterbrechungsfrei durchgeführten mehrstufigen S2-Zugversuch, Bild 6.29). Eine schlüssige Erklärung besteht darin, dass die Zeit, welche zur Anpassung der Versuchssteuerung und der damit zusammen hängenden Arbeitsschritte notwendig ist, bereits zu einer messbaren Relaxation in der Gummischicht 
führt. Die weitergehende Validierung der These war aufgrund der messtechnischen Beschränkungen sowie mangels einer ausreichenden Zahl Probekörper jedoch nicht möglich.

Unter der Annahme von Relaxation haben die quasistätionären Zyklen der unterbrochenen mehrstufigen Versuche bezüglich ihrer jeweiligen Restdeformationen einen größeren Abstand voneinander als die von unterbrechungsfreien Versuchen. Dies wird sich auch auf den identifizierten Parametersatz auswirken, da in den zur Identifikation verwendeten Simulationen die Versuche unterbrechnungsfrei dargestellt werden. Auf eine zusätzliche Modifikation der Messdaten wird dennoch verzichtet, da der tatsächlich korrekte Versatz nicht zweifelsfrei bestimmt werden kann.

Entsprechend Kap. 6.7.2 wird die kardanische Beanspruchung nicht berücksichtigt, sondern dient ausschließlich zur Validierung der Materialparameter. Die Identifikation erfolgt anhand der axialen und radialen Belastung sowie der Torsionsbelastung.

\section{Identifikation für das YEOH-Stoffgesetz}

Auf Basis der Vorbetrachtungen erfolgt die Anpassung anhand der einstufigen Versuche am bauteilnahen Probekörper. Analog zur Homogen-Identifikation wird auf Basis der drei berücksichtigten Versuche die kombinierte gewichtete Fehlerquadrat-Kennzahl GFK ermittelt. Ausgehend von einem willkürlich gewählten Startparametersatz führt die Minimierung von $\overline{\mathrm{GFK}}$ auf die in Tabelle 6.16 angegebenen YEOH-Parameter.

Für die identifizierten Parameter wird die Berechnung der GFK der berücksichtigten Versuche in Tabelle 6.17 dargestellt. Dabei fällt auf, dass die Anpassung an die axiale Belastung deutlich schlechter ist als an die anderen beiden Versuche. Dies belegt auch der visuelle Vergleich der Versuche an den bauteilnahen Probekörpern mit den zugehörigen Simulationen, welche in Bild 6.59 dargestellt sind.

Tabelle 6.16: Ergebnis der YEOH-Parameter-Identifikation nach 6 Schritten.

\begin{tabular}{|l|l|l|l|c|}
\hline & $\mathrm{C}_{10}[\mathrm{MPa}]$ & $\mathrm{C}_{20}[\mathrm{MPa}]$ & $\mathrm{C}_{30}[\mathrm{MPa}]$ & $\overline{\mathrm{GFK}}$ \\
\hline Startparameter & 0,5 & 0,0 & 0,0 & $9,834 \cdot 10^{-3}$ \\
Identifizierte Parameter & 0,463 & $-0,0103$ & 0,000637 & $5,421 \cdot 10^{-3}$ \\
\hline
\end{tabular}

Tabelle 6.17: GFK-Ermittlung für identifizierte YEOH-Parameter.

\begin{tabular}{|l|c|c|c|c|}
\hline & $\sum_{i=1}^{n_{L}}\left(\bar{y}_{i}-\bar{m}_{i}\right)^{2}$ & $n_{L}$ & $\max (\bar{y})-\min (\bar{y})$ & GFK \\
\hline Axiale Belastung & $4,780 \cdot 10^{5}$ & 44 & 1005 & $10,754 \cdot 10^{-3}$ \\
Radiale Belastung & $1,681 \cdot 10^{7}$ & 44 & 10050 & $3,781 \cdot 10^{-3}$ \\
Torsionsbeanspruchung & $2,499 \cdot 10^{8}$ & 44 & 43000 & $3,071 \cdot 10^{-3}$ \\
\hline
\end{tabular}




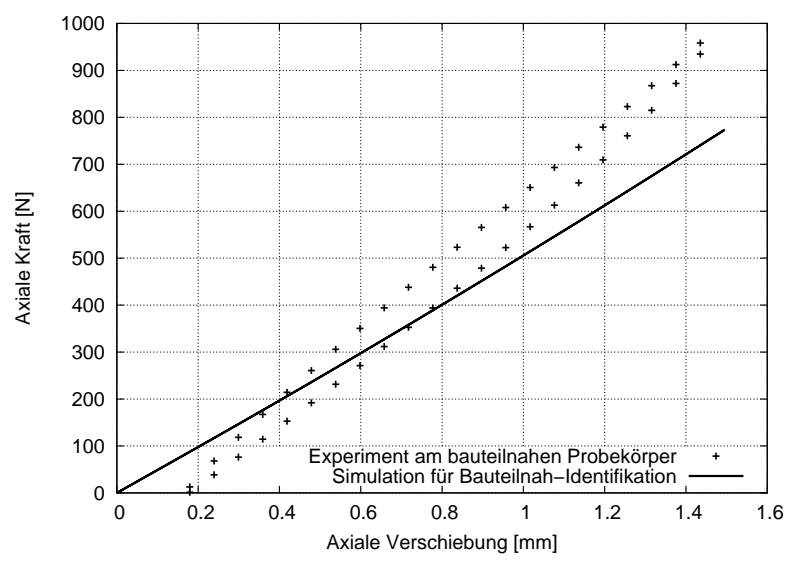

(a) Axiale Belastung

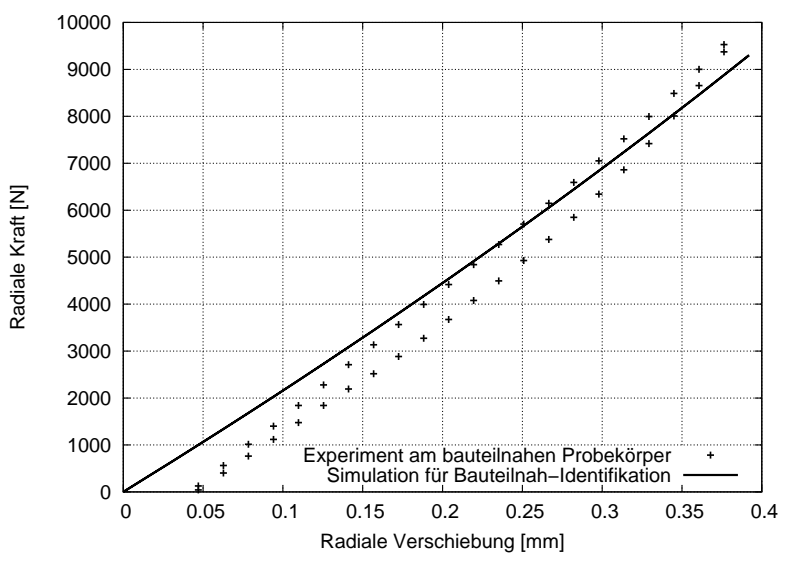

(b) Radiale Belastung

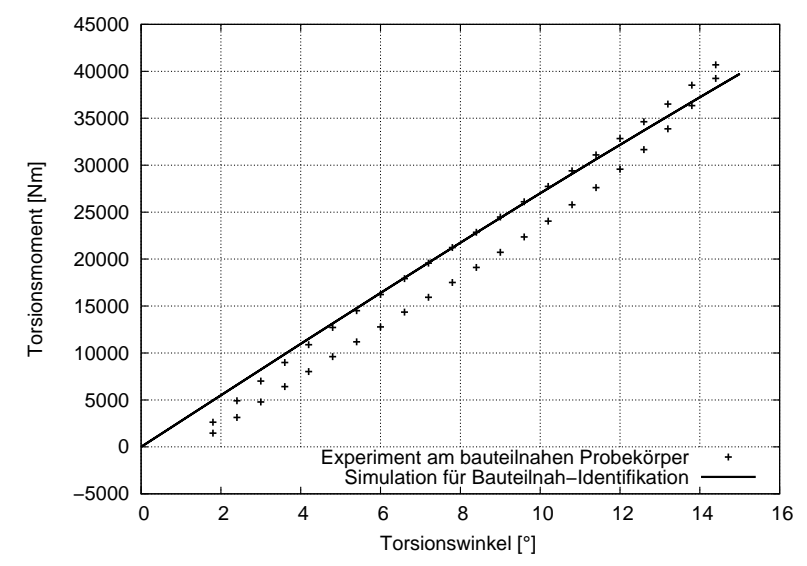

(c) Torsionsbeanspruchung

Bild 6.59: Vergleich der Experimente an bauteilnahen Probekörpern mit den zugehörigen Simulationen bei Verwendung der anhand bauteilnaher Probekörper identifizierten Parameter für YEOH.

Bei Betrachtung der Verläufe für die verschiedenen Arten der Identifikation anhand bauteilnaher Probekörper fällt auf, dass die axiale Belastung in der Simulation zu einem deutlich weicheren Verhalten führt, als es die Experimente tatsächlich zeigen. Werden zusätzlich die Vergleichsdehnungen in Bild 6.54 - Bild 6.56 bzw. die Darstellungen der I1-I2-Diagramme in Bild 6.39 für diese Lastfälle berücksichtigt, lässt sich dieses Verhalten erklären. Bereits in den Vorbetrachtungen zur Identifikation (Kap. 6.6.1) wird gezeigt, dass die Abbildungsgenauigkeit in der Simulation mit vorgegebenen bzw. identifizierten Materialparameter stark von der Höhe der Beanspruchung abhängt. Da die Lastfälle für die Bauteile bzw. bauteilnahen Probekörper bereits im Lastenheft festgelegt sind, ist eine nachträgliche Abstimmung aufeinander nicht möglich. Insbesondere die axiale Belastung führt im Vergleich zu den übrigen Beanspruchungen in großen Bereichen der Gummischicht nur zu geringen Deformationen. Für diese Belastungsbereiche liefert die Identifikation jedoch nur eingeschränkt vertrauenswürdige Materialparameter, da lediglich einstufige Versuche genutzt werden und infolge sowohl radialer Belastung als auch Torsionsbeanspruchung deutlich größere Verzerrungen auftreten. Somit ist die deutliche Abweichung zwischen Experiment und Simulation der axialen Belastung am bauteilnahen Probekörper mit dem identifizierten Parametersatz, welcher einen Kompromiss zwischen den drei berücksichtigten Versuchen darstellt, schlüs- 
sig. Eine mögliche Lösung stellt die Verwendung inelastischer Stoffgesetze dar, welche das tatsächliche Materialverhalten in einem größeren Anwendungsbereich zuverlässig abbilden können.

Mit hyperelastischen Stoffgesetzen ist es nicht möglich, die bei zyklischer Beanspruchung verbleibenden Restdeformationen abzubilden. Diese sind jedoch bei der Fahrwerksbuchse sehr stark ausgeprägt, womit die Anpassungsgenauigkeit bei Verwendung des YEOH-Stoffgesetzes begrenzt ist. Bei einer Anpassung an die einsinnige Erstbelastung eines Probekörpers hingegen spielt dies keine Rolle. Im industriellen Umfeld ist es deshalb an einigen Stellen üblich, die Materialparameter an eben diese Erstbelastungen anzupassen, da dies zu einem deutlich besseren Anpassungsergebnis gegenüber der Anpassung an die quasistationären Zyklen führen soll. Dies ist jedoch schon deshalb nicht zwingend, da bereits die Kalibrierung zu einer deutlichen Änderung der Werkstoffeigenschaften führt und nicht vollständig durch das elastische Materialgesetz abgebildet werden kann. Des Weiteren werden sowohl Be- als auch Entlastungsweg der Hysterese, welche ebenfalls nicht durch das elastische Stoffgesetz abgebildet werden kann, bei Verwendung des kompletten Zyklus berücksichtigt, was bei ausschließlicher Nutzung der Erstbelastung nicht der Fall ist. Zusammenfassend bedeutet dies, dass die Erstbelastung nicht repräsentativ für das Verhalten bei mehrfacher Lastwiederholung ist und die entsprechend identifiziertem Parameter später in der Bauteilsimulation der betrachteten Lastfälle nicht das tatsächliche Materialverhalten im stationären Zustand abbilden. Sinnvoll ist eine solche Anpassung demnach ausschließlich dann, wenn auch in der Bauteilsimulation später lediglich die Darstellung der Erstbelastung relevant ist.

\section{Identifikation für MORPH in RepRicht}

Für die Anpassung an das inelastische Stoffgesetz dienen die in Bild 6.58 dargestellten mehrstufigen Versuche als Grundlage. Der Startparametersatz ist mit dem der HomogenIdentifikation identisch. Die identifizierten Materialparameter sowie die den Lastfällen zugeordneten GFK sind in Tabelle 6.18 und Tabelle 6.19 angegeben.

Tabelle 6.18: Startparameter und anhand bauteilnaher Probekörper bei Minimierung von

$\overline{\text { GFK }}$ für MORPH in RepRicht identifizierte Parameter.

\begin{tabular}{|l|c|c|c|c|c|c|c|c|}
\hline & $p_{1}[\mathrm{MPa}]$ & $p_{2}[\mathrm{MPa}]$ & \multicolumn{1}{|c|}{$p_{3}$} & $p_{4}$ & $p_{5}[\mathrm{MPa}]$ & \multicolumn{1}{|c|}{$p_{6}$} & \multicolumn{1}{c|}{$p_{7}$} & $p_{8}[\mathrm{MPa}]$ \\
\hline$\underline{p}_{\text {Start }}$ & 0,0109 & 0,408 & 0,421 & 6,850 & 0,00560 & 5,54 & 5,84 & 0,1170 \\
$\underline{p}_{\text {Bauteilnah }}$ & 0,1663 & 0,3817 & 0,5939 & 7,603 & 0,000295 & 6,058 & 4,077 & 0,0302 \\
\hline
\end{tabular}

Tabelle 6.19: GFK für die Identifikation anhand bauteilnaher Probekörper bei Minimierung von $\overline{\text { GFK }}$ für MORPH in RepRicht nach 6 Schritten.

\begin{tabular}{|l|c|c|}
\hline & $\operatorname{GFK}\left(\underline{p}_{\text {Start }}\right)$ & $\operatorname{GFK}\left(\underline{p}_{\text {Bauteilnah }}\right)$ \\
\hline Axiale Belastung & $14,63 \cdot 10^{-3}$ & $0,5105 \cdot 10^{-3}$ \\
Radiale Belastung & $14,25 \cdot 10^{-3}$ & $0,6924 \cdot 10^{-3}$ \\
Torsionsbeanspruchung & $3,029 \cdot 10^{-3}$ & $0,5713 \cdot 10^{-3}$ \\
Kombiniert & $9,674 \cdot 10^{-3}$ & $0,5871 \cdot 10^{-3}$ \\
\hline
\end{tabular}


Die gemessenen Größen der für die Identifikation verwendeten quasistationären Zyklen sind in Bild 6.60 grafisch dargestellt. Zudem enthalten die Abbildungen die Verläufe der entsprechenden FEM-Simulationen bei Verwendung der identifizierten Parameter $\underline{p}_{\text {Bauteilnah }}$.

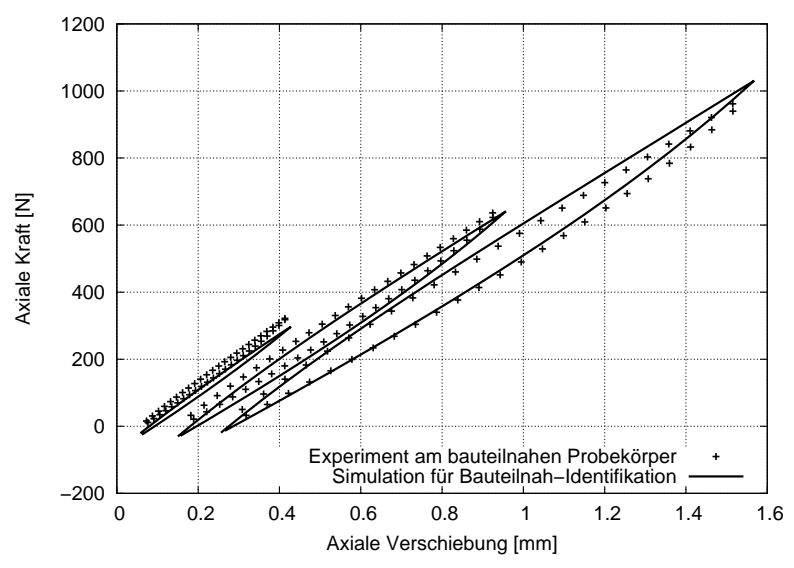

(a) Axiale Belastung

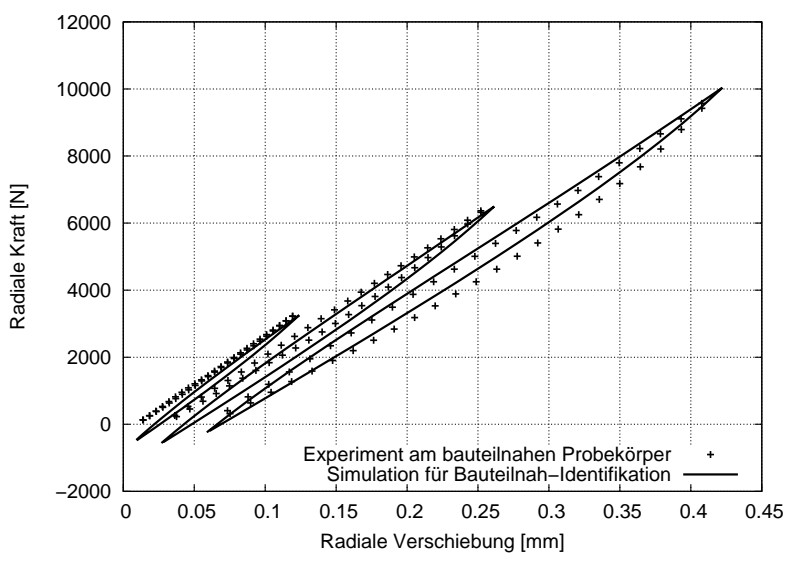

(b) Radiale Belastung

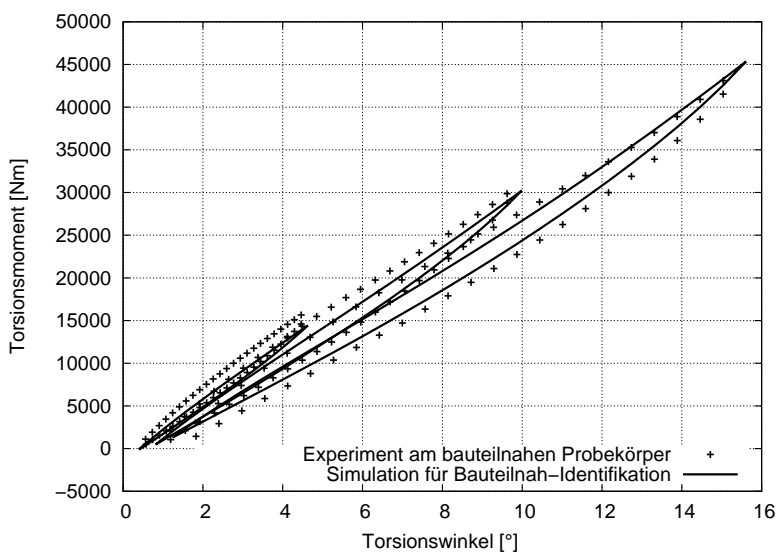

(c) Torsionsbeanspruchung

Bild 6.60: Vergleich der Experimente an bauteilnahen Probekörpern mit den zugehörigen Simulationen bei Verwendung der anhand bauteilnaher Probekörper identifizierten Parameter für MoRPH in RepRicht.

Der gefundene Parametersatz stellt auch hier einen Kompromiss bezüglich der verschiedenen Lastfälle dar. Dies äußert sich darin, dass bei der axialen und radialen Belastung die verbleibende Restdeformation zu groß, bei der Torsionsbelastung jedoch zu klein abgebildet wird. Zudem befinden sich die Fußpunkte der Zyklen bei radialer Belastungen deutlich unterhalb der Nulllinie während sich diese bei Torsion oberhalb befinden. Zur Verbesserung des Ergebnisses im Rahmen zukünftiger Identifikationen erscheinen vor allem zwei Maßnahmen sinnvoll:

- Durchführung der Versuche ohne Unterbrechungen beim Wechsel der Belastungsstufen während der Messungen, auf welche zu Beginn des Kapitels hingewiesen wurde.

- Mehrfache Wiederholung der Versuche für die jeweiligen Lastfälle und anschließende Mittlung der Ergebnisse. Aufgrund der begrenzten Probekörperzahl musste darauf verzichtet werden, womit die Korrektur zufälliger Messfehler bzw. Kompensation von Unterschieden zwischen den geprüften Probekörpern nicht möglich war. 
Dessen ungeachtet können die stationären Zyklen der berücksichtigten Lastfälle mit Hilfe des verwendeten inelastischen Materialgesetzes gut wiedergegeben werden. Zudem zeigt die geringe Anzahl benötigter Optimierungsschritte deutlich, dass die Versuche genügend Informationen zur sicheren Bestimmung der Materialparameter beinhalten.

\subsection{Validierung am Bauteil}

Die Validierung der identifizierten Materialparameter erfolgt anhand der in Kap. 6.5 beschriebenen Bauteilsimulationen. Entsprechend den Vorgaben im Lastenheft werden die verschiedenen Belastungsarten einstufig simuliert und der stationäre Zyklus mit entsprechenden Versuchsergebnissen verglichen. Aufgrund der Tatsache, dass für das OGDEN-Stoffgesetz keine Identifikation anhand bauteilnaher Probekörper durchgeführt wurde, erübrigt sich auch die Berücksichtigung bei der Validierung. Diese beschränkt sich somit auf den Vergleich des YEOH-Stoffgesetzes sowie MORPH in RepRicht für die Identifikation anhand von Standardprobekörpern bzw. bauteilnahen Probekörpern.

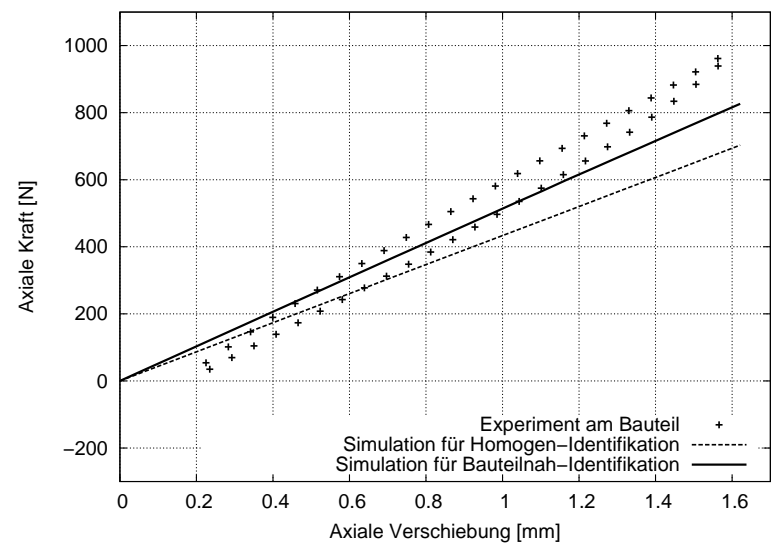

(a) Axiale Belastung

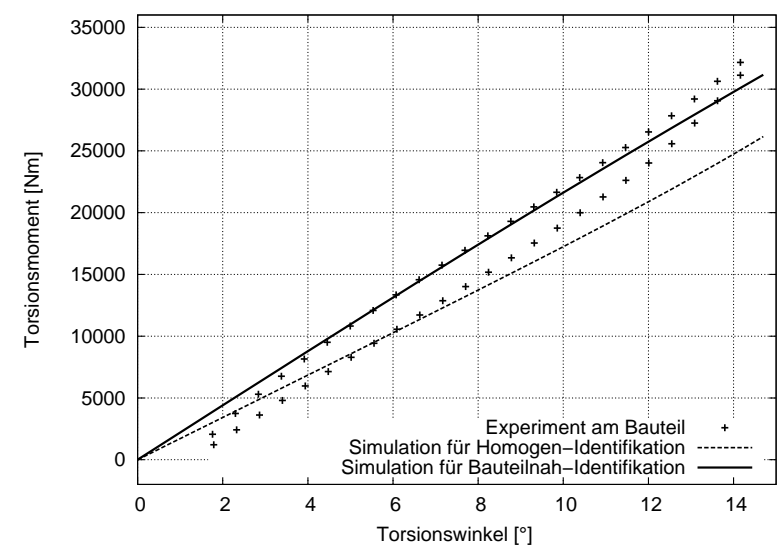

(c) Torsionsbeanspruchung

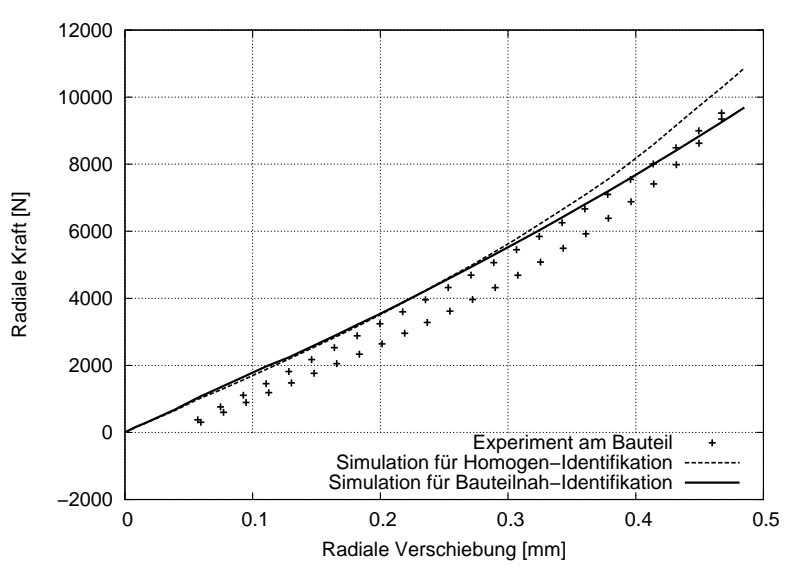

(b) Radiale Belastung

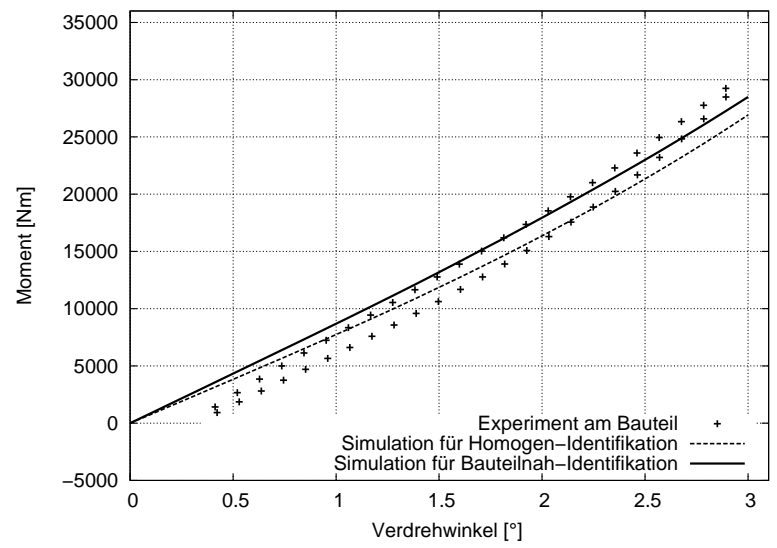

(d) Kardanische Beanspruchung

Bild 6.61: Vergleich der Experimente am realen Bauteil mit den zugehörigen Simulationen bei Verwendung der anhand von homogenen Standardversuchen bzw. bauteilnahen Probekörpern identifizierten Parameter für das YEOH-Stoffgesetz.

In Bild 6.61 ist der Vergleich der Identifikationsergebnisse für YEOH dargestellt. Aufgrund des hyperelastischen Materialverhaltens genügt die Simulation der Erstbelastung, da diese 
mit den Folgezyklen übereinstimmt. Bei Betrachtung der in der Identifikation verwendeten Lastfälle wird deutlich, dass trotz der begrenzten Abbildungsmöglichkeiten des YEOHStoffgesetzes die anhand bauteilnaher Probekörper ermittelten Materialparameter zu besseren Ergebnissen in der Bauteilsimulation führen als die der Homogen-Identifikation (Schellenberg et al., 2012). Lediglich bei der kardanischen Beanspruchung ist kein deutlicher Unterschied vorhanden.

Der Wechsel zum inelastischen MORPH in RepRicht führt bereits bei der Simulation mit Parametern aus der Homogen-Identifikation zu einem erheblich besseren Ergebnis, welche in Bild 6.62 dargestellt sind. Ursache dafür ist insbesondere die Möglichkeit zur Darstellung der Restdeformation bzw. der Hysterese. Werden zur Anpassung der MoRPH-Parameter Versuche an bauteilnahen Probekörpern genutzt, erhöht sich die Abbildungsgenauigkeit der Bauteilsimulation nochmals deutlich (Schellenberg et al., 2013). Dies betrifft vor allem die Breite der Hysterese sowie die Maximalamplituden bei der radialen und kardanischen Belastung.

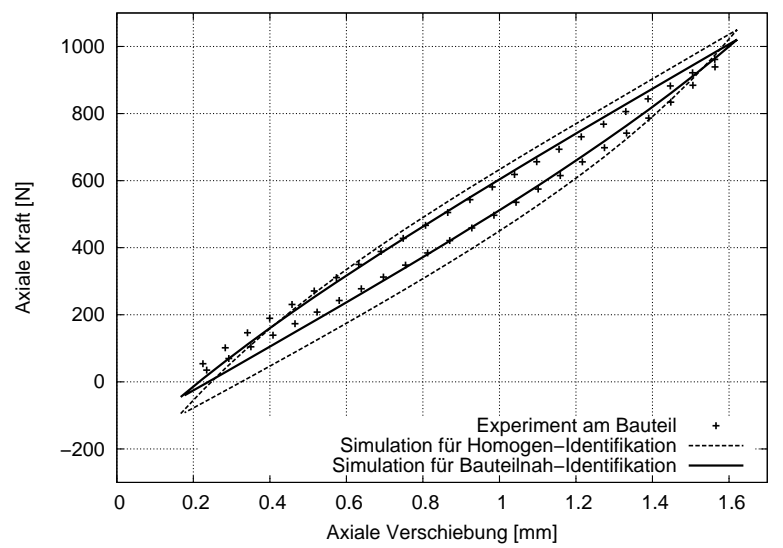

(a) Axiale Belastung

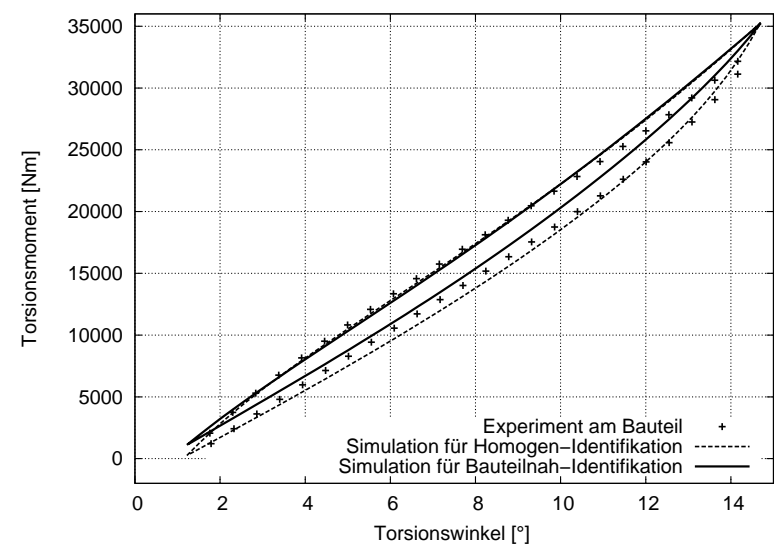

(c) Torsionsbeanspruchung

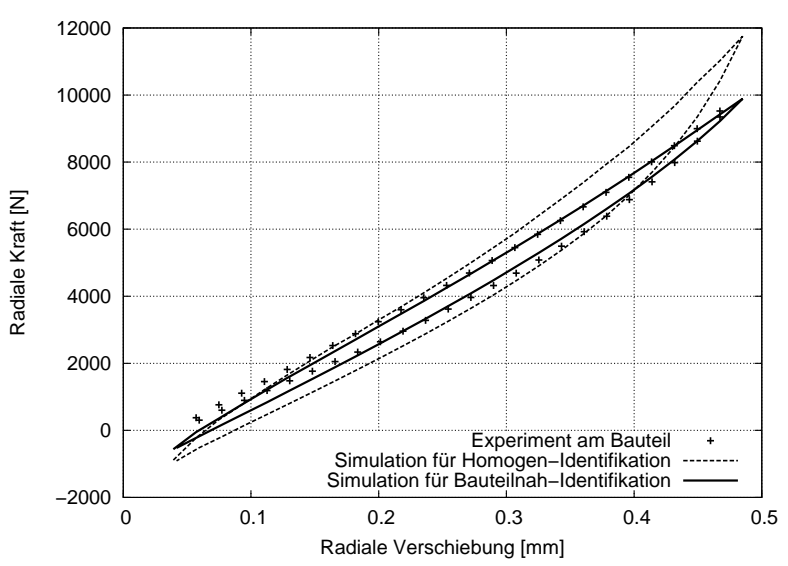

(b) Radiale Belastung

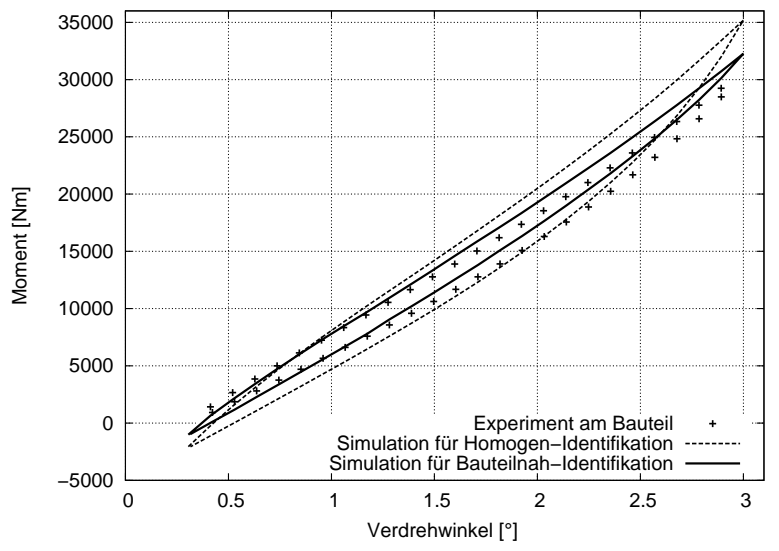

(d) Kardanische Beanspruchung

Bild 6.62: Vergleich der Experimente am realen Bauteil mit den zugehörigen Simulationen bei Verwendung der anhand von homogenen Standardversuchen bzw. bauteilnahen Probekörpern identifizierten Parameter für MORPH in RepRicht.

Besonders die Abbildungsgenauigkeit der kardanischen Belastung des Bauteils zeigt, dass die Informationen bezüglich des Materialverhaltens, welche im Rahmen der Identifikation anhand bauteilnaher Probekörper gewonnen werden, auch für FEM-Simulationen deutlich 
abweichender Belastungsarten geeignet sind. Um die visuelle Darstellung zu untermauern, sind in Tabelle 6.20 die GFK für alle dargestellten Lastfälle sowie die daraus gebildeten $\overline{\text { GFK }}$ zusammengefasst. Darin wird deutlich, dass nicht nur die Art der Identifikation, sondern vor allem die Wahl des Stoffgesetzes für die Darstellungsgenauigkeit der Simulation ausschlaggebend ist.

Tabelle 6.20: GFK zur Bewertung der Abbildungsgenauigkeit der Bauteilsimulationen bei Verwendung der anhand von Standardprobekörpern bzw. bauteilnahen Probekörpern identifizierten Parameter für das YEOH-Stoffgesetz und MORPH in RepRicht.

\begin{tabular}{|l|r|l|l|l|}
\hline & \multicolumn{2}{|c|}{ YEOH-Stoffesetz } & \multicolumn{2}{c|}{ MORPH in RepRicht } \\
& \multicolumn{1}{|c|}{$\underline{p}_{\text {Homogen }}$} & $\underline{p}_{\text {Bauteilnah }}$ & $\underline{p}_{\text {Homogen }}$ & $\underline{\underline{p}}_{\text {Bauteilnah }}$ \\
\hline Axiale Belastung & $19,547 \cdot 10^{-3}$ & $5,890 \cdot 10^{-3}$ & $2,876 \cdot 10^{-3}$ & $0,4479 \cdot 10^{-3}$ \\
Radiale Belastung & $5,002 \cdot 10^{-3}$ & $3,623 \cdot 10^{-3}$ & $3,596 \cdot 10^{-3}$ & $0,4237 \cdot 10^{-3}$ \\
Torsionsbeanspr. & $10,258 \cdot 10^{-3}$ & $2,874 \cdot 10^{-3}$ & $0,3473 \cdot 10^{-3}$ & $0,8492 \cdot 10^{-3}$ \\
Kardanische Beanspr. & $3,169 \cdot 10^{-3}$ & $3,199 \cdot 10^{-3}$ & $3,785 \cdot 10^{-3}$ & $1,141 \cdot 10^{-3}$ \\
Kombiniert & $8,469 \cdot 10^{-3}$ & $3,816 \cdot 10^{-3}$ & $2,346 \cdot 10^{-3}$ & $0,6846 \cdot 10^{-3}$ \\
\hline
\end{tabular}

Darüber hinaus geht aus den Abbildungen hervor, dass sich die Simulationsverläufe beim bauteilnahen Probekörper und beim realen Bauteil trotz deutlicher geometrischer Unterschiede ausgesprochen ähnlich sind. Es ist deshalb zu erwarten, dass auch FEM-Simulationen anderer Fahrwerksbuchsen, die der gleichen Produktgruppe angehören, bei Verwendung der identifizierten Materialparameter zuverlässige Ergebnisse liefern. Dies ist für industrielle Anwendungen besonders interessant, da so die Parameteridentifikation für eine repräsentative Geometrie zur Ermittlung eines Referenz-Parametersatzes genügt.

Zusammenfassend lässt sich konstatieren, dass die Nutzung von Materialparametern, welche an homogene Standardversuche angepasst werden, nur begrenzt vertrauenswürdige Ergebnisse bei FEM-Simulationen am Bauteil ermöglichen. Werden die Parameter hingegen anhand bauteilnaher Probekörper identifiziert, führt dies zu einer deutlich besseren Vorhersage des Bauteilverhaltens im Rahmen einer FEM-Simulation. Es ist jedoch zu berücksichtigen, dass auch die Wahl des Stoffgesetzes einen erheblichen Einfluss besitzt und sich die Auswahl an den im Vorfeld charakterisierten Materialeigenschaften orientieren sollte. 


\section{Zusammenfassung und Ausblick}

Für die Weiterentwicklung technischer Produkte stellt die numerische Simulation auf Basis der Finite-Elemente-Methode ein unverzichtbares Werkzeug dar. Von entscheidender Bedeutung bezüglich der Zuverlässigkeit der Ergebnisse ist nicht zuletzt das gewählte Stoffgesetz und in diesem Kontext die Bestimmung der entsprechenden Materialparameter. Da eine direkte Parameterbestimmung gewöhnlich nicht möglich ist, erfolgt die Identifikation meist durch die Lösung eines inversen Problems der Anpassung an experimentelle Daten. Zwar beinhalten gängige FEM-Programme Verfahren zur Parameteridentifikation, jedoch sind deren Möglichkeiten bezüglich eigener Stoffgesetze bzw. der Optimierungsverfahren begrenzt. Dies gilt in gleichem Maße für die Identifikation geometrischer Formen bzw. der sie beschreibenden Formparameter, die nicht frei zugänglich sind und deshalb ebenfalls der indirekten Bestimmung durch Lösung eines Optimierungsproblems bedürfen.

Bei Aufgaben der Formoptimierung soll die Gestalt eines Bauteils oder Werkzeugs derart verändert werden, dass das bestmögliche Ergebnis für ein definiertes Optimierungsziel gefunden wird. Typische Anwendungsbereiche sind die Minimierung der höchsten Spannungen in einem Bauteil oder der Umformkraft bei Massivumformverfahren wie dem Strangfließpressen. Für die Lösung solcher Probleme stehen ebenfalls verschiedene Werkzeuge zur Verfügung, welche jedoch spezifische Nachteile aufweisen. Diese betreffen vor allem die Einbindung kommerzieller und hauseigener FEM-Programme.

Einen Schwerpunkt der vorliegenden Arbeit stellt deshalb die Entwicklung der Optimierungssoftware SPC-Opt dar, mit welcher sich eine Vielzahl verschiedener Aufgaben aus den Bereichen der Formoptimierung sowie der Material- und Formidentifikation bearbeiten lässt. Die Grundlage hierfür bildet das an der Technischen Universität Chemnitz entwickelte FEM-Forschungsprogramm $S P C-P M 2 A d N l$ und dessen Erweiterung von Lindner (Lindner und Kreißig, 2004), welches die Identifikation von Parametern zur Beschreibung der plastischen Anisotropie metallischer Werkstoffe ermöglicht. Bezüglich der Programmentwicklung ist die Unterstützung durch Wulf (Wulf et al., 2013) hervorzuheben. Zudem finden sich die Ergebnisse mehrerer studentischer Arbeiten in einzelnen Programmmodulen wieder. Das Programm SPC-Opt ist modular aufgebaut und ermöglicht so eine stetige Weiterentwicklung. Zur Lösung von Identifikationsproblemen, welche üblicherweise auf die Minimierung einer Fehlerquadratsumme zurückgeführt werden können, steht eine robuste Implementierung des Levenberg-Marquardt-Fletcher-Verfahrens zur Verfügung. Ergänzt wird dieses durch eine Reihe verschiedener Line-Search- und Trust-Region-Verfahren. Letztere eignen sich besonders zur Bearbeitung von Aufgaben der Formoptimierung. In diesem Kontext wurden zudem effiziente Algorithmen zur Approximation der Hesse-Matrix integriert. Ergänzend stehen verschiedene Verfahren zur Startparametervariation zur Verfügung, da zwar meist das globale Minimum einer Zielfunktion gesucht wird, die vorhandenen auf Gradienten basierenden Verfahren jedoch manchmal nur ein lokales Minimum liefern. Die Implementierung eines Baukastensystems zur Erstellung der Zielfunktion, ein automatisches Log-System 
und die konsequente Nutzung von XML-Ein- und Ausgabeformaten erleichtern die Bedienung und ermöglichen in Form einer Programmauskopplung die Anwendung in der Industrie.

Einen essentiellen Bestandteil der Optimierung stellt die Berechnung der Zielfunktionswerte dar. Dies kann auf verschiedene Weise erfolgen - durch Nutzung von Skripten, mathematischen Programmen oder hauseigenen bzw. kommerziellen FEM-Programmen. Eine große Bedeutung kommt deshalb der Implementierung geeigneter Schnittstellen zur Einbindung dieser Programme zu. Die Funktionalität einer solchen Schnittstelle beinhaltet das Einlesen und die Ausgabe der Eingabedateien sowie die Fähigkeit, dem zur Berechnung des Funktionswertes verwendeten Programm die bezüglich der örtlichen und zeitlichen Diskretisierung notwendigen Informationen zu übermitteln. Zum aktuellen Zeitpunkt werden die kommerzielle FEM-Software ABAQUS, ANSYS und MSC.MARC, verschiedene hauseigene FEM-Programme sowie LUA-Skripte unterstützt.

Bei der Identifikation von Formparametern sowie der Formoptimierung werden während der Optimierung geometrische Konturen gezielt verändert. Voraussetzung hierfür ist deren Parametrisierung mit Hilfe geeigneter Designvariablen. Aus diesem Grund wurde in SPC-Opt die Funktionalität implementiert, vorhandene Konturen wie Linien, Splines oder KeypointGruppen mit Hilfe von NURBS zu approximieren und die Koordinaten und Wichtungsfaktoren der zugehörigen Kontrollpunkte als Formparameter zu nutzen. Damit verbunden war zudem die Implementierung eines analytischen Algorithmus zur Aktualisierung der FEMNetze entsprechend der Formparameteränderung zugehöriger Konturen. Um negative Folgen stark verzerrter Netze infolge der Netzaktualisierung zu vermeiden, wurden zusätzlich verschiedene Algorithmen zur automatischen Netzglättung integriert.

Die technische Entwicklung der letzten Jahre im Bereich der Computertechnik hat die Nutzung paralleler Rechentechniken praktisch unverzichtbar gemacht. Bei der Entwicklung von SPC-Opt wurde dem Rechnung getragen, indem auf zwei Ebenen die Möglichkeit zur Parallelisierung implementiert wurde. So können die FEM-Simulationen durch Nutzung der jeweiligen programmeigenen Möglichkeiten parallelisiert werden, wobei dies meist durch die verfügbaren Lizenzen begrenzt wird. Darüber hinaus ist es möglich, die notwendigen Funktionswertberechnungen eines Optimierungsschrittes in beliebiger Form auf mehreren Instanzen von SPC-Opt aufzuteilen und auch auf diese Weise die absolute Rechenzeit zu verringern.

Neben der Programmierung der Software stellt die praktische Anwendung im industriellen Umfeld den zweiten Schwerpunkt der Arbeit dar. Dieser bezog sich auf die Weiterentwicklung bestehender Verfahren zur Materialparameteridentifikation im Bereich der Gummiwerkstoffe, deren besondere Materialeigenschaften Ursache für die Verwendung in einer Vielzahl technischer Produkte sind. Um das inelastische Materialverhalten, welches sich in charakteristischen Effekten wie Hysterese, Entfestigung oder bleibender Verformung äußert, in FEM-Simulationen abbilden zu können, wurden in der jüngeren Vergangenheit eine Reihe hochwertiger Stoffgesetze entwickelt. Dem gegenüber hat sich die Vorgehensweise zur Identifikation der Stoffparameter nur unwesentlich weiterentwickelt. Die übliche Methode der Anpassung an homogene Standardversuche ignoriert die Tatsache, dass das tatsächliche Materialverhalten und somit auch die Materialparameter des gewählten Stoffgesetzes eines Bauteils nicht nur von der chemischen Zusammensetzung, sondern auch von der Bauteilgeometrie, dem Fertigungsprozess und nicht zuletzt von der Belastungsgeschichte abhängen. 
Um die Unterschiede bezüglich Geometrie und Fertigungsprozess zwischen dem Bauteil und dem Probekörper zu verringern, wurde von Ihlemann (Ihlemann, 2003) als Lösungsansatz das Konzept der Anpassung anhand bauteilnaher Probekörper entwickelt. Basierend auf diesem Ansatz wurde im Rahmen der vorliegenden Arbeit am Beispiel einer Fahrwerksbuchse ein Probekörper entworfen, welcher dem originalen Bauteil zwar ähnlich sieht, jedoch eine deutlich einfachere Geometrie hat. Dadurch konnte das Verhalten des Bauteils gut approximiert und dennoch sichergestellt werden, dass die im Rahmen der Parameteridentifikation durchgeführten FEM-Simulationen sicher konvergieren. Das verwendete Material sowie der Fertigungsprozess von Bauteil und Probekörper sind identisch. Auch die Belastungen der zur Identifikation verwendeten Versuche orientierten sich an den im Lastenheft definierten Beanspruchungen. Zur Identifikation wurde eine auf industrielle Bedürfnisse zugeschnittene Auskopplung von SPC-Opt verwendet. Die Simulation der Lastfälle erfolgte mit den kommerziellen FEM-Programmen $A B A Q U S^{*}$ und $M S C . M A R C^{*}$, deren entsprechende Schnittstellen in $S P C$-Opt bezüglich ihrer Funktionalität erweitert wurden. Dies war vor allem deshalb notwendig, da das im Projekt genutzte MorPH-Stoffgesetz (Besdo und Ihlemann, 2003) in den FEM-Programmen nur über die Nutzerschnittstellen implementiert werden konnte.

Während des Projektverlaufs erfolgten weitere Betrachtungen zur Parameteridentifikation. Hierfür wurden zu Vergleichszwecken auch herkömmliche Versuche an Standardprobekörpern durchgeführt. In diesem Kontext erfolgte die Untersuchung und Bewertung typischer Vorgehensweisen zur Manipulation von Messdaten in der Industrie und ihre Auswirkungen auf die Identifikation. Zur besseren Vergleichbarkeit verschiedener homogener Lastfälle konnte ein einfach zu berechnendes, auf dem Umformgrad basierendes, Vergleichsmaß hergeleitet werden. Da sich die bei der Berechnung der Zielfunktion berücksichtigten Belastungsarten bezüglich Belastungstyp, Lastschrittzahl und Belastungsintensität unterscheiden, wurde die "Gewichtete Fehlerquadrat-Kennzahl" entwickelt. Dadurch können die verschiedenen Versuche quantitativ miteinander verglichen werden.

Für die FEM-Simulation einiger Belastungen am realen Bauteil, und eingeschränkt auch am bauteilnahen Probekörper, traten Konvergenzschwierigkeiten infolge entartender Elemente auf. Durch die Implementierung sogenannter "Stabilisierungselemente" konnte das Entarten vermieden werden und die FEM-Simulationen konvergierten erfolgreich. Dadurch war die Validierung der bauteilnah identifizierten Parameter anhand des Vergleiches der Experimente am Bauteil und der zugehörigen FEM-Simulationen möglich. Die Validierung bestätigte, dass die Verwendung bauteilnah identifizierter Parameter zu einer erheblich besseren Abbildung des Materialverhaltens führt als die Verwendung anhand von Standardprobekörpern identifizierter Parameter. Es zeigte sich weiterhin, dass vor allem der Einsatz eines Stoffgesetzes mit der Möglichkeit zur Abbildung des charakteristischen Verhaltens von Elastomeren unbedingt erforderlich ist.

Dennoch bleiben einige Fragestellungen ungeklärt, z.B. inwieweit ein für ein spezifisches Bauteil identifizierter Parametersatz auch zuverlässige Simulationsergebnisse für Bauteile mit einer ähnlichen Geometrie liefert. Die Möglichkeit zur "Wiederverwendbarkeit" identifizierter Parametersätze hätte einen erheblichen Effizienzgewinn zur Folge, weshalb die Klärung dieser Thematik das Teilziel eines Nachfolgeprojektes darstellt.

${ }^{*}$ Detaillierte Angaben sind im Anhang [A.1 aufgeführt. 
Auch die allgemeine Annahme eines temperatur- und belastungsunabhängigen Kompressionsmoduls ist, unabhängig vom verwendeten Stoffgesetz, sehr fraglich. Dies belegen sowohl alte als auch neue Arbeiten (Adams und Gibson, 1930; Stommel und Zimmermann, 2012). Da die Wahl des Kompressionsmoduls einen großen Einfluss auf das gesamte Materialverhalten in der FEM-Simulation hat, ist die nähere Betrachtung im Rahmen eines gesonderten Projektes sehr sinnvoll.

Im Hinblick auf die Weiterentwicklung von $S P C$-Opt ist die bereits laufende Integration von SQP-Verfahren zur Lösung restingierter Probleme zu nennen. Die Vielfalt möglicher Anwendungen erweitert sich dadurch deutlich, insbesondere für den Bereich der Formoptimierung. Dies liegt daran, dass Restriktionen einzelner Designvariablen bzw. davon abhängiger Größen zur Einhaltung definierter Vorgaben bezüglich Bauraum, Masse, Volumen, Spannungen usw. deutlich besser in der Optimierung berücksichtigt werden können.

Die Frage, wie stark sich die Variation einzelner Parameter auf das Modell und somit auf die Zielfunktion auswirkt, kann bislang in SPC-Opt nicht zufriedenstellend beantwortet werden. Insbesondere für Probleme der Formoptimierung ist es jedoch bereits während der Modellerstellung wichtig, die optimale Anzahl und Position derjenigen Designvariablen zu ermitteln, welche die zu optimierenden Konturen beschreiben. Vor diesem Hintergrund erscheint eine entsprechende Erweiterung des Funktionsumfangs sehr sinnvoll, zumal verschiedene anwendungsabhängige Verfahren bereits existieren (Saltelli et al., 2008).

Grundsätzlich ist der Aufwand zur Entwicklung hauseigener Optimierungssoftware wiederkehrend zu hinterfragen. Dies ist vor allem dem Fakt geschuldet, dass kommerzielle Anbieter von CAD- und FEM-Programmen sowie Optimierungssoftware stetig die Funktionalität ihrer Produkte erweitern. Dadurch werden die Anwendungsbereiche zunehmend erweitert und die Vorteile spezialisierter Lösungen abgeschwächt oder gar kompensiert. Verstärkt wird dies durch den fortschreitenden Konzentrationsprozess in Form von Übernahmen oder Fusionen, beispielsweise die Übernahme der Firmen Simufact, Free Field Technologies (FFT) u.w. durch MSC Software.

Die im Rahmen der vorliegenden Arbeit entwickelte Optimierungssoftware $S P C$-Opt ist nicht nur in der aktuellen Form ein vielseitiges Werkzeug zur Bearbeitung von Problemstellungen der Identifikation und Formoptimierung. Dank der modularen Struktur kann die Software entsprechend neuer Anforderungen flexibel erweitert werden und stellt daher auch in weiteren Forschungsprojekten einen wichtigen Bestandteil dar. Durch gezielte Modifikationen der Optimierungsverfahren bezüglich Robustheit und Automatisierung entstand eine Programmauskopplung, welche auch im industriellen Umfeld einfach anzuwenden ist und selbst bei schlecht konditionierten Problemen der Parameteridentifikation gute Ergebnisse mit wenigen Optimierungsschritten ermöglicht. In diesem Kontext ist auch die Programmierung von Schnittstellen zur Einbindung verschiedener kommerzieller FEM-Programme bei Verwendung eigener Stoffgesetze hervorzuheben. Diese wurden gezielt im Hinblick auf ein stabiles Konvergenzverhalten trotz stark verzerrter FE-Netze sowie geringe Geschwindigkeitsverluste infolge des notwendigen Datenaustauschs zwischen dem FEM-Programm und SPC-Opt optimiert. Im Ergebnis dessen sind die beteiligten Industriepartner nunmehr in der Lage, die Vorteile fortgeschrittener Materialmodelle im Rahmen von FEM-Simulationen bzw. bei der Lösung von Optimierungsaufgaben effizient und sicher zu nutzen. 


\section{Anhang}

\section{A.1 Software und Programmpakete}

An mehreren Stellen der vorliegenden Arbeit wird auf Software und Programmpakete unterschiedlichen Umfangs verwiesen. Im Folgenden sind diese aufgelistet und mit weiterführenden Informationen versehen. Die Angaben zur Programmversion beinhalten keine Aussage bezüglich der im Text angegebenen Funktionalität. Sie dienen ausschließlich zur Information darüber, welche Versionen im Rahmen der Arbeit betrachtet bzw. verwendet wurden.

\section{ABAQUS}

Programmbezeichnung: Abaqus

Entwickler: Dassault Systèmes

Version: 6.11-6.14

URL: http://www.3ds.com/products-services/simulia/products/abaqus

\section{ADOL-C}

Programmbezeichnung: ADOL-C und weitere Programmpakete

Entwickler: COIN-OR

Version: ADOL-C 2.5

URL: http://www.coin-or.org/projects/ADOL-C.xml

\section{ALGLIB}

Programmbezeichnung: ALGLIB

Entwickler: ALGLIB Project

Version: 3.8.1

URL: http://www.alglib.net/

\section{ANKHOR}

Programmbezeichnung: ANKHOR FlowSheet

Entwickler: ANKHOR Software GmbH

Version: 2.0.1

URL: http://www.ankhor.com

\section{ANSA}

Programmbezeichnung: ANSA

Entwickler: BETA CAE Systems S.A.

Version: 15.0

URL: http://www.beta-cae.gr/ansa.htm 


\section{ANSYS}

Programmbezeichnung: ANSYS Mechanical

Entwickler: ANSYS, Inc.

Version: 14.0

URL: http://www.ansys.com/Products/Simulation+Technology/Structural+Analysis/

ANSYS+Mechanical

\section{BLITZ}

Programmbezeichnung: Blitz ++ Library

Entwickler: oonumerics

Version:

URL: http://www.oonumerics.org/blitz/

\section{CARAT ++}

Programmbezeichnung: CARAT ++ (vormals CARAT)

Entwickler: FEMopt Studios

Version: CARAT-4

URL: http://www.femopt.de/index.php/de

\section{CMAKE}

Programmbezeichnung: CMake

Entwickler: Bill Hoffman, Ken Martin, Brad King, Dave Cole, Alexander Neundorf, Clinton Stimpson

Version: 2.8 .12

URL: http://www.cmake.org/

\section{DOXYGEN}

Programmbezeichnung: Doxygen

Entwickler: Dimitri van Heesch

Version: 1.6.1

URL: http://www.stack.nl/ dimitri/doxygen/index.html

\section{FT-Optimization}

Programmbezeichnung: FEMtools Optimization

Entwickler: Dynamic Design Solutions

Version: 3.8

URL: http://www.femtools.com/products/ftopt.htm

\section{HYPERMESH}

Programmbezeichnung: Altair HyperMesh

Entwickler: Altair HyperWorks

Version: 12.0

URL: http://www.altairhyperworks.de/ 


\section{LS-OPT}

Programmbezeichnung: LS-OPT

Entwickler: DYNAmore

Version: 4.2

URL: http://www.dynamore.de/de/produkte/opt/ls-opt

\section{MATLAB}

Programmbezeichnung: MATLAB (Optimization Toolbox, Global Optimization Toolbox)

Entwickler: MathWorks

Version: R2013-R2014

URL: http://www.femtools.com/products/ftopt.htm

\section{MSC.MARC}

Programmbezeichnung: Marc

Entwickler: MSC Software

Version: 2007-2013

URL: http://www.mscsoftware.com/de/product/marc

\section{MSC.NASTRAN}

Programmbezeichnung: Nastran

Entwickler: MSC Software

Version: 2013

URL: http://www.mscsoftware.com/de/product/msc-nastran

NX

Programmbezeichnung: NX(vormals UNIGRAPHICS)

Entwickler: Siemens PLM Software

Version: 10

URL: http://www.plm.automation.siemens.com/de_de/products/nx/10/index.shtml

\section{OptiStruct}

Programmbezeichnung: OptiStruct

Entwickler: Altair

Version: 11.0

URL: http://www.altairhyperworks.de/Product,19,OptiStruct.aspx

\section{PATRAN}

Programmbezeichnung: Patran

Entwickler: MSC Software

Version: 2012

URL: http://www.mscsoftware.com/de/product/patran

\section{POCO}

Programmbezeichnung: POCO $\mathrm{C}++$ Libraries

Entwickler: Günter Obiltschnig, Aleksandar Fabijanic u.w.

Version: 1.3

URL: http://pocoproject.org/ 


\section{PTC/Creo}

Programmbezeichnung: PTC Creo Parametric(vormals Pro/ENGINEER Wildfire)

Entwickler: INNEO Solutions

Version: Pro/Engineer Wildfire 5.0

URL: http://de.ptc.com/product/creo/3d-cad/parametric

\section{SOLIDWORKS}

Programmbezeichnung: SolidWorks Simulation

Entwickler: Dassault Systèmes

Version: 2013-2014

URL: http://www.solidworks.de/sw/products/simulation/structural-optimization.htm

\section{SPC-PM2AdNl}

Programmbezeichnung: SPC-PM2AdNl

Entwickler: Institut für Mechanik und Therodynamik, Professur Numerische Mathematik, TU-Chemnitz

Version: 2004

URL: http://www.tu-chemnitz.de/sfb393

\section{SVN}

Programmbezeichnung: Apache Subversion

Entwickler: The Apache Software Foundation

Version: 1.6 .11

URL: http://subversion.apache.org/

\section{TOSCA}

Programmbezeichnung: Tosca Structure

Entwickler: Dassault Systèmes (bis 2013 FE-Design)

Version: 7.0.2

URL: http://www.fe-design.de 


\section{A.2 Visualisierung von FEM-Ergebnissen}

Die ansprechende Visualisierung der Ergebnisse von FEM-Simulationen ist sowohl für die Präsentation wissenschaftlicher Erkenntnisse bei Konferenzen als auch für die Darstellung in Artikeln und wissenschaftlichen Arbeiten wie der vorliegenden Dissertation unerlässlich. Leider entspricht die vorhandene Qualität der mit kommerziellen FEM-Programmen erzeugten Grafiken und Animationen nur eingeschränkt einem hohen Anspruch und es häufig nicht möglich, fehlerfreie Vektorgrafiken zu erzeugen bzw. ohne akzeptablen Qualitätsverlust in Präsentationen zu integrieren.

Aufgrund dessen wurde in Zusammenarbeit mit Sigmund (2000) eine auf ANKHOR * basierende Anwendung entwickelt, welche aus einer Bibliothek mit Operatoren zum Einlesen, Manipulieren und visuellen Aufbereiten von Ergebnisdaten aus FEM-Programmen sowie einer darauf aufbauenden interaktiven Dashboard-Anwendung besteht. Die Bedienoberfläche dieses im Weiteren als "FEM-Viewer" bezeichneten Programmes ist in Bild A.1 anhand eines Beispielmodells dargestellt.

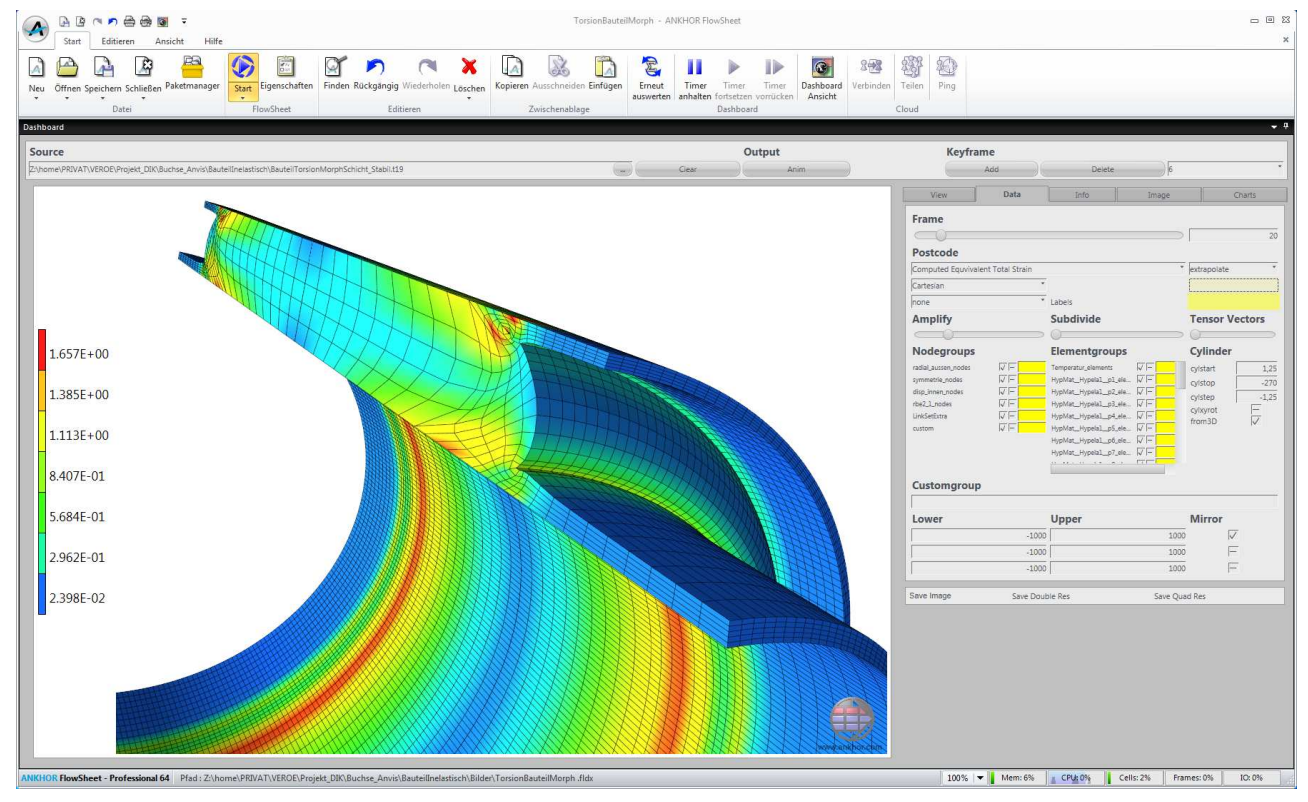

Bild A.1: Darstellung der Bedienoberfläche des FEM-Viewers.

Mit Hilfe des FEM-Viewers ist es möglich, die Ergebnisse einer FEM-Simulation einzulesen und sie für Präsentationen oder andere Anwendungsbereiche aufzubereiten. Kenntnisse bezüglich des zugrunde liegenden Programmes ANKHOR sind hierfür nicht erforderlich. Der grundsätzliche Ablauf bei der Generierung qualitativ hochwertiger Bilder beinhaltet das Einlesen der zu visualisierenden FEM-Daten, die Anpassung der Anzeigeeinstellungen sowie die anschließende Speicherung für die oben genannten Anwendungsfälle. Hierbei werden die nachfolgenden Dateiformate unterstützt:

- vektorbasiertes WMF bzw. EMF als Exportformat für MS Office-Anwendungen, z.B. PowerPoint

*Detaillierte Angaben sind im Anhang [A.1 aufgeführt. 
- PNG als Bitmap basiertes Format, z.B. für Web-Präsentationen

- EPS als Vektorformat für die Druckausgabe oder zur Integration in TeX-basierten Veröffentlichungen

- PDF zur Weitergabe der Daten via Email

Darüber hinaus können auf Keyframes basierende Animationen generiert und mit automatischer Kompression in ein übliches Videoformat wie z.B. MPEG-2 umgewandelt werden.

Bei der Programmentwicklung war zu beachten, dass die bei einer FE-Analyse generierten Ergebnisdaten nicht immer in einem anzeigefreundlichen Format vorliegen (vergl. Kap. 2.4). Zudem ist es teilweise notwendig, die Ergebnisse durch Rotation (z.B. Axialsymmetrie), Extrusion (z.B. bei Vorliegen eines EVZ) oder Spiegelung (z.B. bei Nutzung von Symmetrierandbedingungen) auf entsprechende 3D-Darstellungen zu erweitern. Zusätzlich müssen die in den Gaußpunkten ermittelten Simulationsergebnisse durch Interpolation bzw. Extrapolation oder durch Eigenwertbestimmung auf die Eckpunkte der finiten Elemente transformiert werden.

Um Ausgabegrößen unterschiedlicher Simulationen des gleichen Bauteils gut miteinander vergleichen zu können und den Arbeitsaufwand zu verringern, kann eine gewählte BauteilPerspektive bzw. ein definierter Satz Bildparameter zur Darstellung unterschiedlicher Ergebnisdateien verwendet werden. Für die Visualisierung der FE-Modelle hinsichtlich der ermittelten Verformungen und Spannungen existieren zahlreiche Möglichkeiten zur Hervorhebung von Details, z.B. die Modifikation der Farbe oder Strichstärke von Elementkanten.

In der aktuellen Form können die Ergebnisse des FEM-Programms $M S C . M A R C^{*}$ in Form der auf FORTRAN basierenden "t19" -Dateien eingelesen und verarbeitet werden. Eine Herausforderung stellt hierbei die Tatsache dar, dass die Ergebnisdaten teilweise mehrere Gigabyte umfassen und dadurch nicht als Ganzes im Arbeitsspeicher vorgehalten werden können. Aus diesem Grund erfolgt das Parsen der Daten dynamisch, was bedeutet, dass nur die aktuell benötigten Teile der Ergebnisdatei eingelesen werden.

*Detaillierte Angaben sind im Anhang [A.1] aufgeführt. 


\section{Literaturverzeichnis}

Adams, L. und Gibson, R., 1930: The compressibility of rubber. J. Acad. Sci. 20, S. 213-223.

Alexander, H., 1968: A Constitutive Relation for Rubber-Like Materials. International Journal of Engineering Science 6, S. 549-563.

Alt, W., 2011: Nichtlineare Optimierung : eine Einführung in Theorie, Verfahren und Anwendungen. Wiesbaden: Vieweg + Teubner.

Ausze, P. und Eglais, V., 1977: New approach for planning out of experiments. Problems of Dynamic and Strength 35, S. 104-107.

Beck, J. und Arnold, K., 1977: Parameter Estimation in Engineering and Science. New York: Wiley.

Benedix, U., 2000: Parameterschätzung für elastisch-plastische Deformationsgesetze bei Berücksichtigung lokaler und globaler Vergleichsgrößen. Dissertation, TU Chemnitz, Chemnitz: Inst. f. Mechanik.

Bergström, J. und Boyce, M., 1998: Constitutive Modelling of the Large Strain TimeDependent Behavior of Elastomers. J. Mech. Phys. Solids 46, S. 931-954.

Besdo, D. und Ihlemann, J., 2003: A phenomenological constitutive model for rubberlike materials and its numerical applications. Int. Journal of Plasticity 19 (7), S. 1019-1036.

Bhatti, M. A., 2000: Practical Optimization Methods. New York: Springer-Verlag.

Blacker, T. und Stephenson, M., 1991: Paving: A new approach to automated quadrilateral mesh generation. International Journal for Numerical Methods in Engineering 32(4), S. 811-847.

Bohnsack, E., 1997: Zur Identifikation von Verfestigungsparametern elastisch-plastischer Stoffgesetze an inhomogen verformten Proben. Dissertation, TU Chemnitz, Chemnitz: Inst. f. Mechanik.

Bonnans, J.; Gilbert, J.; Lemaréchal, C. und Sagastizábal, C., 1997: Optimisation Numérique, Aspects théoreques et pratiques. New York: Springer-Verlag.

de Boor, C., 2001: A practical guide to splines. New York: Springer-Verlag. 
Bos, A. v. d., 2007: Parameter Estimation for Scientists and Engineers. John Wiley and Sons.

Branets, L. und Carey, G., 2005: A local cell quality metric and variational grid smoothing algorithm. Engineering with Computers 6, S. 19-28.

Bronstein, I.; Semendjajew, K.; Musiol, G. und Mühlig, H., 2005: Taschenbuch der Mathematik. Frankfurt a.M.: Verlag Harri Deutsch, 6 Ed..

Broyden, C., 1970: The convergence of a class of double rank minimization algorithms: 2. The new algorithm. J. Inst. Math. Appl. 6, S. 222-231.

Bucher, A., 2001: Deformationsgesetze für große elastisch-plastische Verzerrungen unter Berücksichtigung einer Substruktur. Dissertation, TU Chemnitz, Chemnitz: Inst. f. Mechanik.

Burkardt, J., 2008: Random Points on a Uniform Grid. Florida State University, School of Computational Science, http://people.scs.fsu.edu/ burkardt/cpp_src/grid/grid.html.

Canann, S.; Stephenson, M. und Blacker, T., 1993: Optismoothing: An optimization-driven approach to mesh smoothing. Finite Elements in Analysis and Design 13(2-3), S. 185-190.

Canann, S.; Tristano, J. und Staten, M., 1998: An approach to combined Laplacian and optimization-based smoothing for triangular, quadrilateral and tetrahedral meshes. Proceedings of the 7th International Meshing Roundtable 7, S. 479-494.

Chen, Z.; Tristano, J. und Kwok, W., 2003: Combined Laplacian and optimization-based smoothing for quadratic mixed surface meshes. Proceedings of the 12th International Meshing Roundtable 12, S. 361-370.

Clausner, A., 2007: Anwendung von Line-Search-Strategien zur Formoptimierung und Parameteridentifikation. Diplomarbeit(unveröff.), TU Chemnitz, Institut für Mechanik und Thermodynamik.

Dargazany, R. und Itskov, M., 2009: A network evolution model for the anisotropic Mullins effect in carbon black filled rubbers. International Journal of Solids and Structures 46, S. $2967-2978$.

Dennis, J. und Schnabel, R., 1996: Numerical Methods for unconstrained optimization and nonlinear equations. Philadelphia: Siam Verlag.

Elias, H., 2009: Makromoleküle Band 1-4. John Wiley and Sons.

Elsässer, B., 1998: Approximation mit rationalen B-Spline Kurven und Flächen. Dissertation, RWTH Aachen, Shaker Verlag. 
Fang, K.; Lin, D.; Winker, P. und Zhang, Y., 2000: Uniform Designs: Theory and Application. Technometrics 42(3), S. 237-248.

Fang, K.; Lu, X. und Winker, P., 2003: Lower bounds for centered and wrap-around $L_{2}{ }^{-}$ discrepancies and construction of uniform designs by threshold accepting. Journal of Complexity 19, S. 692-711.

Fang, K.; Li, R. und Sudjianto, A., 2006: Design and Analysis for Computer Experiments. Chapman \& Hall/CRC.

Fiacco, A., 1983: Introduction to Sensitivity and Stability Analysis in Nonlinear Programming. New York: Academic Press.

Fletcher, R., 1970: A new approach to variable metric algorithms. Computer J. 13, S. 317 322.

Fletcher, R., 1971: A modified Marquardt subroutine for non-linear least squares. Report of the Theoretical Physics Division, AERE-R 6799.

Floriani, L. und Spagnuolo, M., 2008: Shape Analysis and Structuring. Berlin Heidelberg: Springer-Verlag.

Freund, M., 2013: Verallgemeinerung eindimensionaler Materialmodelle für die FiniteElemente-Methode. Dissertation, TU Chemnitz, Düsseldorf: VDI.

Freund, M. und Ihlemann, J., 2010: Generalization of one-dimensional material models for the finite element method. Zeitschrift für Angewandte Mathematik und Mechanik 90 (5), S. 399-417.

Friedrich, L., 2011: Untersuchung von Flächenapproximationsverfahren mit NURBS-Flächen im Rahmen der 3D-Formoptimierung. Studienarbeit(unveröff.), TU Chemnitz, Institut für Mechanik und Thermodynamik.

Gamma, E.; Helm, R.; Johnson, R. und J., V., 1994: Theorie und Numerik restringierter Optimierungsaufgaben. Prentice Hall.

Geiger, C. und Kanzow, C., 2002: Theorie und Numerik restringierter Optimierungsaufgaben. Heidelberg: Springer-Verlag.

Gent, A., 1962: Relaxation Processes in Vilcanized Rubber. I. Relation among Stress Relaxation, Creep, Recovery, and Hysteresis. J. Polym. Science 22, S. 433-441.

Gent, A. und Thomas, A. G., 1958: Forms of the stored (strain) energy function for vulcanized rubber. J. Polym. Science 28, S. 625-637.

Gill, P. E.; Murray, W. und Wright, M. H., 1981: Practical Optimization. London: Academic Press. 
Giunta, A.; Wojtkiewicz Jr., S. F. und Eldred, M. S., 2003: Overview of modern design of experiments methods for computational simulations. In: Proceedings of the 41st AIAA Aerospace Sciences Meeting and Exhibit, American Institute of Aeronautics and Astronautics.

Goldfarb, D., 1970: A family of variable metric methods derived by variational means. Math. Comp. 24, S. 23-26.

Grary, S., 2002: Design of Computer Experiments for Metamodel Generation. Analog Integrated Circuits and Signal Processing 32, S. 7-16.

Grewolls, G., 1998: Numerische Untersuchungen zur anisotropen plastischen Verfestigung unter besonderer Berücksichtigung der Verhältnisse in gewalzten Blechen. Dissertation, TU-Chemnitz, Chemnitz: Inst. f. Mechanik.

Gross, D., 1992: Bruchmechanik 1: Grundlagen, Lineare Bruchmechanik. Berlin Heidelberg: Springer-Verlag.

Grossmann, K. W., 1997: Ein neues Verfahren zur globalen Optimierung und dessen Anwendung in der Automatisierungstechnik. Dissertation, Universität Kaiserslautern, Shaker Verlag.

Göktepe, S. und Miehe, C., 2005: A Micro-Macro Approach to Rubber-like Materials. Journal of the Mechanics and Physics of Solids. Part III: The Micro-Sphere Model of Anisotropic Mullins-Type Damage 53, S. 2259-2283.

Hart-Smith, L., 1966: Elasticity parameters for finite deformations of rubber-like materials. Z. angew. Math. Phys. 17, S. 608-626.

Harzheim, L., 2008: Strukturoptimierung, Grundlagen und Anwendungen. Frankfurt a.M.: Verlag Harri Deutsch.

Haupt, P., 2002: Continuum Mechanics and Theory of Materials. New York: Springer-Verlag.

Hennessy, J. und Patterson, D., 2003: Computer Architecture: A Quantitative Approach Third Edition. San Francisco: Morgan Kaufman Publishers.

Hickernell, F., 1998: Lattice rules: how well do they measure up? In: Random and QuasiRandom Point Sets (Edited by P. Hellekalek und G. Larcher), New York: Springer-Verlag, S. 106-166.

Hofmann, W. und Gupta, H., 2010: Handbuch der Kautschuk-Technologie - Band 1-3. H. Gupta.

Hohl, C., 2007: Anwendung der Finite-Elemente-Methode zur Parameteridentifikation und Bauteilsimulation bei Elastomeren mit Mullins-Effekt. Dissertation, Universität Hannover, Düsseldorf: VDI. 
Husslage, B., 2006: Maximin designs for computer experiments. Dissertation, Center for Economic Research, Tilburg University.

Ierusalimschy, R., 2006: Programmieren mit Lua. Open Source Press.

Ihlemann, J., 2003: Kontinuumsmechanische Nachbildung hochbelasteter Gummiwerkstoffe. Dissertation, Universität Hannover, Düsseldorf: VDI.

Ihlemann, J., 2007: Generalization of one-dimensional constitutive models with the concept of representative directions. Boukamel, A., Laiarinandrasana, L., Mo, S., Verron, E.: Constitutive Models for Rubber V, S. 29-34.

Johnson, M.; Moore, L. und Ylvisaker, D., 1990: Minimax and maximin distance designs. Journal of Statistical Planning and Inteference 26, S. 131-148.

Kirkpatrick, S.; Gelatt, Jr. C.D. und M.P., V., 1983: Optimization by Simulated Annealing. Science 220(4598), S. 671-680.

Klüppel, M. und Schramm, M., 2000: A generalized tube model of rubber elasticity and stress softening of filler reinforced elastomer systems. Macromolecular Theory and Simulations 9, S. $742-754$.

Knupp, P., 2002: Algebraic mesh quality metrics. SIAM Journal of Scientific Computing 23(1), S. 193-218.

Koehler, J. und Owen, A., 1996: Computer Experiments. In: Handbook of Statistics, 13: Design and Analysis of Experiments (Edited by C. Rao und S. Ghosh), North-Holland, S. 261-308.

Kreißig, R.; Benedix, U.; Görke, U.-J. und Lindner, M., 2007: Identifikation and estimation of constitutive parameters for material laws in elastoplasticity. GAMM-Mitt. 30, S. 458-480.

Landgraf, R., 2008: Kombination von stochastischen und deterministischen Verfahren zur Lösung von Optimierungsaufgaben der Mechanik. Diplomarbeit(unveröff.), TU Chemnitz, Institut für Mechanik und Thermodynamik.

Lastovetsky, A., 2003: Parallel Computing on Heterogenous Networks. Hoboken New Jersey: Wiley-Interscience.

Levenberg, K., 1944: A method in the solution of certain non-linear problems in least squares. Q. Appl. Math. 2, S. 164-168.

Lindner, M., 2010: Ermittlung der plastischen Anisotropie durch Eindringversuche. Dissertation, TU Chemnitz, Chemnitz: Inst. f. Mechanik u. Thermodynamik. 
Lindner, M. und Kreißig, R., 2004: Determination of the plastic anisotropy of metals by indentation tests - Part B: Hill's modified theory for finite deformations and its implementation into the FEM-research-program SPC-PM2AdNI. Proceedings of the 4th ICFG Workshop on Process Simulation. In: Proceedings of the 4 th ICFG Workshop on Process Simulation (Shanghai), S. 10-16.

Lion, A., 1996: A constitutive model for carbon black filled rubber: experimental investigations and mathematical representation. Continuum Mechanics and Thermodynamics 8, S. 153169 .

Lion, A., 1997: On the large deformation behaviour of reinforced rubber at different temperatures. J. Mech. Phys. Solids 45, S. 1805-1834.

Lion, A., 2000: Thermomechanik von Elastomeren: Experimente und Materialtheorie. Habilitationsschrift, Universität Kassel, Kassel: Inst. f. Mechanik.

Luenberger, D., 1997: Optimization by Vector Space Methods. New York: Wiley Professional.

Mahnken, R. und Stein, E., 1996: Parameter identification for viscoplastic models based on analytical derivatives of a least-squares functional and stability investigations. Int. J. Plast. 12, S. 451-479.

Marquardt, D., 1963: An Algorithm for Least-Squares Estimation of Nonlinear Parameters. SIAM J. Appl. Math. 11, S. 431-441.

McKay, M. C.; J., B. R. und Conover, W. J., 1979: A Comparison of Three Methods for Selecting Values of Input Variables in the Analysis of Output from a Computer Code. Technometrics 21(2), S. 239-245.

Miehe, C. und Göktepe, S., 2005: A Micro-Macro Approach to Rubber-like Materials. Journal of the Mechanics and Physics of Solids. Part II: The Micro-Sphere Model of Finite Rubber Viscoelasticity 53, S. 2231-2258.

Miehe, C.; Göktepe, S. und Lulei, F., 2004: A Micro-Macro Approach to Rubber-like Materials. Part I: The Non-Affine Micro-Sphere Model of Rubber Elasticity. Journal of the Mechanics and Physics of Solids 52, S. 2617-2660.

Miller, R., 2000: Optimization. John Wiley and Sons.

Mooney, M., 1940: A Theory of Large Elastic Deformation. Journal of Applied Physics 11.

Moritz, H., 1993: General Considerations Regarding Inverse and Related Problems. Inverse Problems: Principles and Applications in Geophysics, Technology and Medicine 74, S. 11.

Morris, M. und Mitchell, T., 1995: Exploratory designs for computational experiments. Journal of Statistical Planning and Inference 43, S. 381-402. 
Mukherjee, N., 2002: A Hybrid, Variational 3D Smoother For Orphaned Shell Meshes. Proceedings of the 11th International Meshing Roundtable 11, S. 379-390.

Nagdi, K., 2004: Gummi-Werkstoffe: Ein Ratgeber für Anwender. H. Gupta.

Nocedal, J. und Wright, S. J., 1999: Numerical Optimization. New York: Springer-Verlag.

Nostitz, N., 2014: Simulation von Kontaktkräften unter Berücksichtigung von Adhäsion und nachgiebigen Oberflächenrauigkeiten. Diplomarbeit (unveröff.), TU Chemnitz, Professur Festkörpermechanik / Professur Numerische Mathematik.

Ogden, R., 1972: Large deformation isotropic elasticity - on the correlation of theory and experiment for incompressible rubberlike solids. Proc. Royal Soc. London A326 , S. 565584.

Ogden, R. und Roxburgh, D., 1999: An energy-based model of the Mullins effect. Constitutive Models for Rubber III - Proceedings of the 3rd European Conference on Constitutive Models for Rubber, S. 23-28.

Panhans, S., 2006: Ein viskoplastisches Materialmodell mit nichtquadratischer Fließfunktion. Dissertation, TU Chemnitz, Chemnitz: Inst. f. Mechanik u. Thermodynamik.

Parhami, B., 2005: Computer Architecture: From Microprocessors To Supercomputers. Oxford: Oxford University Press.

Park, J., 1994: Optimal Latin-hypercube designs for computer experiments. Journal of Statistical Planning and Interference 39, S. 95-111.

Piegl, L. und Tiller, W., 1995: The NURBS Book. Berlin Heidelberg: Springer-Verlag.

Rao, S. S., 2009: Engineering Optimization: Theory and Practice. John Wiley and Sons.

Röthemeyer, F. und Sommer, F., 2001: Kautschuktechnologie. München: Hanser Fachbuch.

Saltelli, A.; Ratto, M.; Andres, T.; Campolongo, F.; Cariboni, J.; Gatelli, G.; Saisana, M. und Tarantola, S., 2008: Global Sensitivity Analysis - The Primer. John Wiley and Sons.

Santer, T.; Williams, B. und Notz, W., 2003: The Design an Analysis of Computer Experiments. New York: Springer-Verlag.

Schellenberg, D. und Kreißig, R., 2009: Optimierung von Werkzeugen und Prozessparametern bei Massivumformverfahren. DFG-Abschlussbericht (unveröff.).

Schellenberg, D.; Lindner, M. und Kreißig, R., 2007: Nonlinear Optimization in Context with Parameter Identification and Shape Optimization. In: Application Stochastics and Optimization Methodes, ETH Zürich, Forming Technology Forum 07, S. 55-60. 
Schellenberg, D.; Kreißig, R. und Ihlemann, J., 2010: Optimierungsstrategien im Bereich der Formoptimierung bei Massivumformverfahren. Proceedings in Applied Mathematics and Mechanics (PAMM) 10, S. 543-544.

Schellenberg, D.; Ihlemann, J. und Juhre, D., 2012: Parameter identification based on multiple inhomogeneous experiments of practical relevance. Constitutive Models for Rubber VII - Proceedings of the 7th European Conference on Constitutive Models for Rubber 136, S. $33-38$.

Schellenberg, D.; Ihlemann, J. und Juhre, D., 2013: Identification of inelastic material parameters using component-oriented specimen. Constitutive Models for Rubber VIII - Proceedings of the 8th European Conference on Constitutive Models for Rubber , S. 191-196.

Schneider, F.-J., 1993: Interpolation, Approximation und Konvertierung mit rationalen BSplines. Dissertation, RWTH Aachen, Shaker Verlag.

Schnur, D. und Zabaras, N. J., 1992: An inverse method for determining elastic material properties and a material interface. Int. J. Num. Eng. 33, S. 2039-2057.

Schumacher, A., 2005: Optimierung mechanischer Strukturen - Grundlagen und industrielle Anwendungen. Berlin Heidelberg: Springer-Verlag.

Shanno, D., 1970: Conditioning of quasi-Newton methods for function minimization. Math. Comp. 24, S. 647-650.

Shannon, C., 1948: A Mathematical Theory of Communication. Bell System Technical Journal 27, S. 379-423 und 623-656.

Shewry, M. und Wynn, H., 1987: Maximum entropy sampling. Journal of Applied Statistics 14(2), S. 165-170.

Sigmund, U., 2000: Entwurf und Evaluierung mehrfädig superskalarer Prozessortechniken im Hinblick auf Multimedia. Dissertation, TU Karlsruhe, Institut für Rechnerentwurf und Fehlertoleranz.

Silc, J.; Robic, B. und Ungerer, T., 1999: Processor Architecture: From Dataflow to Superscalar and Beyond. Berlin Heidelberg: Springer-Verlag.

Snir, M., 1998: MPI: The Complete Reference - Volume 1/2. The MIT Press.

Sorenson, H., 1980: Parameter Estimation. New York: Marcek Dekker.

Stommel, M. und Zimmermann, J., 2012: Determination of the behaviour of rubber components under hydrostatic pressure. Constitutive Models for Rubber VII - Proceedings of the 7th European Conference on Constitutive Models for Rubber. 136, S. 397-401. 
Thielecke, F., 1997: Parameteridentifizierung von Simulationsmodellen für das viskoplastische Verhalten von Metallen - Theorie, Numerik, Anwendungen. Dissertation, TU Braunschweig, Braunschweig: Mechanik-Zentrum.

Thompson, J.; Soni, B. und Weatherill, N., 1998: Handbook of Grid Generation. CRC Press.

Törn, A. und Žilinskas, Z., 1989: Global Optimization. Lecture Notes in Computer Science 350, Berlin Heidelberg: Springer-Verlag.

Ulbricht, V. und Röhle, H., 1975: Berechnung von Rotationsschalen bei nichtlinearem Deformationsverhalten. Dissertation, TU Dresden, Fakultät für Maschinenwesen.

Wulf, H.; Schellenberg, D. und Ihlemann, J., 2012: Simultaneous identification of material and geometry parameters in view of structured specimen. Proceedings in Applied Mathematics and Mechanics (PAMM) 12, S. 759-760.

Wulf, H.; Schellenberg, D.; Kreißig, R. und Ihlemann, J., 2013: Combined Shape and Parameter Identification Applied to a Porous Media Simulation. Technische Mechanik 33(1), S. 67-84.

Ye, K.; Li, W. und Sudjianto, A., 2000: Algorithmic construction of optimal symmetric Latin hypercube designs. Journal of Statistical Planning and Inference 90, S. 145-159.

Yeoh, O., 1990: Characterization of elastic properties of carbon-black filled rubber vulcanizates. Rubber Chemistry and Technology 63, S. 792-805.

Yeoh, O. H., 1993: Some forms of the strain energy function for rubber. Rubber Chemistry and technology 66, S. 754-771. 